\title{
Reclassification of the doryctine tribe Rhaconotini (Hymenoptera, Braconidae)
}

\author{
Sergey A. BELOKOBYLSKIJ ${ }^{1, *}$ \& Alejandro ZALDÍVAR-RIVERÓN ${ }^{2}$ \\ ${ }^{1}$ Zoological Institute Russian Academy of Sciences, St. Petersburg, 199034, Russia. \\ ${ }^{1}$ Museum and Institute of Zoology Polish Academy \\ of Sciences, Wilcza 64, Warszawa 00-679, Poland. \\ ${ }^{2}$ Colección Nacional de Insectos, Instituto de Biología, Universidad \\ Nacional Autónoma de México, $3^{\text {er }}$ Circuito Exterior s/n, Cd. \\ Universitaria, Copilco, Coyoacán, A.P. 70-233, C.P. 04510, Ciudad de México, México. \\ *Corresponding author: doryctes@gmail.com \\ 22Email: azaldivar@ib.unam.mx \\ ${ }^{1}$ urn:1sid:zoobank.org:author:13EDEDEF-68BA-430B-8FC3-0096874859AB \\ ${ }^{2}$ urn:lsid:zoobank.org:author:B6E09990-6CA5-403F-AC3F-9498DBB5BEDF
}

\begin{abstract}
The classification of the genera belonging to the doryctine tribe Rhaconotini (Braconidae) is updated. The following new taxa are described: Troporhaconotus gen. nov. (with 12 species), Afroipodoryctes subgen. nov. (of Ipodoryctes Granger, 1949) (with three species), Hexarhaconotinus subgen. nov. (of Rhaconotinus Hedqvist, 1965) (with ten species), Bathycentor zimbabwensis sp. nov., Ipodoryctes (Afroipodoryctes) reunionus sp. nov., I. (A.) saintphilippensis sp. nov., Platyspathius (Platyspathius) venezuelicus sp. nov., P. (P.) ranomafanus sp. nov., Rhacontsira haeselbarthi sp. nov., $R h$. mozambiquensis sp. nov., Rh. saigonensis sp. nov. and Rh. toamasina sp. nov. The generic status of Euryphrymnus Cameron, 1910 is resurrected. The generic name Aptenobracon Marsh, 1965 is synonymised under Rhaconotus Ruthe, 1854 (syn. nov.); Rhaconotus asiaticus Belokobylskij, 1990 is synonymised under Rh. kerzhneri Belokobylskij, 1985 (syn. nov.). The new name, Rhaconotinus austrochinensis nom. nov., is suggested for the preoccupied name Rhaconotus chinensis Chen \& Shi, 2004 (December) not Rhaconotus chinensis Belokobylskij \& Chen, 2004 (June). New species contents, in many cases with numerous new combinations, are suggested for the genera Bathycentor Saussure, 1892, Euryphrymnus Cameron, 1910, Ipodoryctes Granger, 1949, Rhaconotinus Hedqvist, 1965 and Rhaconotus. The tribe Leptorhaconotini is synonymised with Rhaconotini based on previously published molecular phylogenetic studies, though we leave this group within the subtribe Leptorhaconotina.
\end{abstract}

Keywords. Rhaconotini, taxonomy, description, new combination, synonyms.

Belokobylskij S.A. \& Zaldívar-Riverón A. 2021 Reclassification of the doryctine tribe Rhaconotini (Hymenoptera, Braconidae). European Journal of Taxonomy 741: 1-168. https://doi.org/10.5852/ejt.2021.741.1289 


\section{Introduction}

The doryctine tribe Rhaconotini (Braconidae) was originally erected by Fahringer (1928) to contain the genus Rhaconotus Ruthe, 1854 (Shenefelt \& Marsh 1976), whose known geographic distribution currently comprises the five continents (Yu et al. 2016; Jasso-Martínez et al. 2019). More recently, based on examination of external morphological features, with emphasis on the condition of the metasomal tergites of the type and numerous additional Rhaconotus species, Belokobylskij (1992, 2000, 2001) changed the Rhaconotini to a subtribe level (Rhaconotina) within the tribe Doryctini, including in it four additional genera: the Nearctic Aptenobracon Marsh, 1965, the Oriental and Australasian Arhaconotus Belokobylskij, 2000, the Oriental Mimipodoryctes Belokobylskij, 2000 and Ipodoryctes Granger, 1949, which occurs in the Afrotropical and Oriental regions. Two taxa were subsequently added to this subtribe, the Australasian Neorhaconotus Belokobylskij, Iqbal \& Austin, 2004 (Belokobylskij et al. 2004) and the Afrotropical Grangerdoryctes Belokobylskij, 2005.

A molecular phylogenetic study carried out for the Doryctinae (Zaldívar-Riverón et al. 2008) elevated the Rhaconotini as a valid tribe based on the inclusion of species of Ipodoryctes, Mimipodoryctes and Rhaconotus which were recovered in a separate clade together with four additional, morphologically less similar genera: Antespathius Belokobylskij, 1995, Leptorhaconotus Granger, 1949, Platyspathius Viereck, 1911 and Rhacontsira Belokobylskij, 1998. Moreover, two additional genera, the Western Palaearctic Pseudorhaconotus van Achterberg \& Shaw, 2010 (van Achterberg \& Shaw 2010) and the Afrotropical Bathycentor Saussure, 1892 (Belokobylskij 2018), were later proposed to belong to the above tribe. This generic composition of the Rhaconotini was recently confirmed by a comprehensive molecular phylogenetic study that included a vast number of representative species of the tribe (JassoMartínez et al. 2019).

The main, though not universal, diagnostic external morphological features for members of Rhaconotini are: 1) usually more or less distinctly enlarged fifth or sixth tergites which are hardly sclerotised, often sculptured at least basally and with separated laterotergites; 2) fourth-sixth tergites usually with transverse crenulate subbasal furrows; 3) femora of legs often with dorsal protuberances; 4) parallel vein (CU1a) of fore wing usually interstitial or at least arising before (upper) middle of distal vein (3CU1) of brachial (first subdiscal) cell; 5) recurrent vein (m-cu) of fore wing mostly postfurcal, but rarely interstitial or weakly antefurcal; and 6) second radiomedial vein ( $\mathrm{r}-\mathrm{m})$ of fore wing mainly present, but sometimes (Grangerdoryctes and Pseudorhaconotus) absent. Our comprehensive examination of specimens belonging to all Rhaconotini genera, however, revealed a considerable variation of the above diagnostic characters, including some important features used to diagnose genera within the tribe, such as number of visible tergites and position of recurrent $(\mathrm{m}-\mathrm{cu})$ and parallel $(2 \mathrm{CUb})$ veins of fore wing.

The biological information for members of the Rhaconotini is rather limited in comparison to its number of described species. According to a recently published summary of biological information for the group (Jasso-Martínez et al. 2019), species of this tribe with known host records are idiobiont ectoparasitoids mainly of stem-borer, xylophagous and seed-feeding larvae of the coleopteran families Anobiidae, Bostrichidae, Buprestidae and Curculionidae, and more rarely Chrysomelidae and Mordellidae. However, some mainly Afrotropical and Oriental species have been reared from stem-borer or leafand shoot-feeding larvae of the lepidopteran families Crambidae, Gelechiidae, Pyralidae (all important crop pests) and rarely Cossidae and Noctuidae (the record for Brachodidae was erroneous) (Shenefelt \& Marsh 1976; Belokobylskij \& Maetó 2009; Yu et al. 2016). Hosts still are unknown for species of Antespathius, Arhaconotus, Bathycentor, Grangerdoryctes, Leptorhaconotus, Mimipodoryctes, Neorhaconotus, Pseudorhaconotus, Rhacontsira and the genus that is described here, Troporhaconotus gen. nov. 
Here we provide an updated generic level classification for the Rhaconotini, which is proposed following the above relationships recovered from the phylogenetic study by Jasso-Martínez et al.'s (2019). We formally establish the composition of the tribe to be composed of 13 genera with the inclusion of the new genus Troporhaconotus gen. nov., which is erected here to comprise the species of Rhaconotus with six or more visible metasomal tergites. Moreover, some of the species that were originally included within Rhaconotus s. lat. are here transferred to Ipodoryctes Granger, 1949 since they were nested with members of this genus. The generic status of Rhaconotinus Hedqvist, 1965 and Euryphrymnus Cameron, 1910 stat. resurr. is also confirmed or restored, respectively, and Aptenobracon Marsh, 1965 is synonymised with Rhaconotus Ruthe, 1854.

\section{Material and methods}

The terminology employed for morphological features and measurements follows Belokobylskij \& Maetô (2009). The wing venation nomenclature follows Belokobylskij \& Maetô (2009), with van Achterberg (1993) terminology shown in parentheses. The suture between second and third metasomal tergites is named here as 'second suture'.

Specimens were examined using an Olympus SZX12 stereo microscope. Photographs were taken with a Leica IC 3D digital camera mounted on a Leica MZ16 microscope and using the Leica Application Suite imaging system (Museum and Institute of Zoology, Warsaw, Poland), and with a Canon EOS 70D digital camera mounted on an Olympus SZX10 microscope (Zoological Institute RAS, St Petersburg, Russia). Digital pictures were enhanced, and plates were edited using Adobe Photoshop ${ }^{\circledR}$.

The specimens examined for this study, including type material, are deposited in the following collections:

$\begin{array}{ll}\text { ANIC } & =\text { Australian National Insect Collection, Canberra, Australia } \\ \text { BMNH } & =\text { Natural History Museum, London, UK } \\ \text { CNCI } & \text { Canadian National Collection of Insects, Ottawa, Canada } \\ \text { HNHM } & \text { Hungarian Natural History Museum, Budapest, Hungary } \\ \text { IB-UNAM } & \text { Colección Nacional de Insectos, Instituto de Biología, Universidad Nacional } \\ \text { MHBG } & =\begin{array}{l}\text { Mutónoma de México, Mexico } \\ \text { MIZW }\end{array} \\ \text { MNCN } & \text { forschung, Berlin, Germany } \\ \text { MNHN } & =\text { National Museum of Natural Sciences, Madrid, Spain } \\ \text { NMS } & =\text { Muséum National d'Histoire Naturelle, Paris, France } \\ \text { SDEI } & =\text { Senckenberg Deutsches Entomologisches Institut, Müncheberg, Germany } \\ \text { SSMG } & =\text { Zoologische Staatssamlung, Munich, Germany } \\ \text { TAMU } & =\text { Texas A\&M University, College Station, USA } \\ \text { USNM } & =\text { United States National Museum, Washington, USA } \\ \text { ZISP } & =\text { Zoological Institute of the Russian Academy of Sciences, St Petersburg, Russia } \\ \text { ZMHU } & =\text { Zoological Museum, Helsinki University, Helsinki, Finland }\end{array}$

\section{Abbreviations used for the world's biogeographic regions}
$\mathrm{AF}=$ Afrotropical region
$\mathrm{AU}=$ Australasian region
$\mathrm{NA}=$ Nearctic region
$\mathrm{NT}=$ Neotropical region
$\mathrm{OR}=$ Oriental region
$\mathrm{PA}=$ Palaearctic region
$\mathrm{PC}=$ Pacific region 


\begin{abstract}
Abbreviations used in descriptions
$\mathrm{POL}=$ postocellar line, shortest distance between posterior ocelli

$\mathrm{OD}=$ maximum diameter of the posterior ocellus

$\mathrm{OOL}=$ ocular-ocellar line, shortest distance between posterior ocellus and eye
\end{abstract}

\title{
Results
}

Class Insecta Linnaeus, 1758

Order Hymenoptera Linnaeus, 1758

Family Braconidae Nees, 1811

Subfamily Doryctinae Foerster, 1863

Tribe Rhaconotini Fahringer, 1928

Rhaconotini Fahringer, 1928: 8.

Leptorhaconotini van Achterberg, 1984: 42, syn. nov.

Leptorhaconotini - Belokobylskij 1992: 920 - Quicke 1996: 115 - Yu et al. 2016.

\section{Diagnosis}

Body medium sized to large, but rarely (Grangerdoryctes) small, with surface mostly granulate or granulate-reticulate, metasoma occasionally partly smooth, especially in posterior half. Hypoclypeal depression round or oval. Malar suture usually absent, and sometimes present but usually very shallow. Occipital carina upper usually distinct and complete, below often not reaching base of mandible and obliterated here. Scapus of antenna simple, without basal constriction and apical lobe.

Pronotal collar usually short, dorsally often convex and with distinct pronotal carina. Mesoscutum usually gently-roundly elevated above pronotum; mesoscutum with distinct and complete or rarely incomplete (present only anteriorly) notauli. Prepectal (epicnemial) carina complete, rather strong, sometimes ventrally coarse and wide. Propodeum without or with areas (at least basolateral ones) delineated by distinct carinae, areola present or absent.

Predominantly full-winged, but rarely (some Rhaconotus) present micropterous or apterous forms. Fore wing often hyaline, but sometimes maculated or infuscate. Pterostigma rather wide, only rarely (Grangerdoryctes) narrowed. Second radiomedial (submarginal) cell usually medium-sized. Second radiomedial (r-m) usually present, but rarely absent second (Grangerdoryctes, Pseudorhaconotus) or first radiomedial (2RS) (Antespathius) veins. Recurrent (m-cu) vein often postfurcal (distal to first radiomedial vein (2RS)), more rarely interstitial or at least weakly antefurcal (Rhacontsira, some species of Ipodoryctes and Rhaconotus). Parallel vein (CU1a) often interstitial, rarely not interstitial (Bathycentor, Ipodoryctes, Mimipodoryctes, Rhacontsira) and arising before, from or behind middle of the vein (2CUa) closed brachial (subdiscal) cell posteriorly. Hind wing recurrent vein (m-cu) usually present, but often weakly sclerotised, sometimes absent. Submedial (subbasal) cell always closed distally by nervellus (cu-a), mostly short (except Neorhaconotus), with first mediocubital abscissa $(\mathrm{M}+\mathrm{CU})$ distinctly shorter than second abscissa (1M).

Hind coxa mostly with basoventral corner and tubercle. Hind femur often with distinct basoventral knob.

Metasoma often with five (Pseudorhaconotus, Rhaconotus) or six (Arhaconotus, many Ipodoryctes, Mimipodoryctes, many Rhaconotinus, Troporhaconotus) dorsally visible, hardly sclerotised and widely sculptured tergites, accordingly fifth or sixth tergite in such case more or less enlarged and often longer than previous ones. In some cases (Antespathius, Grangerdoryctes, Platyspathius, Rhacontsira) apical 
tergites less strongly sclerotised, with more tergites visible behind fifth (seven or eight) and usually much less sculptured in distal half of metasoma. Laterotergites (epipleura) of second and following tergites usually distinctly separated and spiracle situated on lateral parts of tergites near folds. First metasomal tergite often medium length or weakly elongate, with short acrosternite (basal sternite); but sometimes (Antespathius and Platyspathius) this tergite long and distinctly narrowed with more or less strongly elongate acrosternite and this segment transformed in (sub)petiole. Second tergite without any furrows (Antespathius, Platyspathius, some Rhaconotus, Rhacontsira) or often with rather distinct curved or straight subbasal furrow (delineated anterior, basal area) (Arhaconotus, Bathycentor, Ipodoryctes, Mimipodoryctes) and subposterior furrow (delineated posterior apical area) (Arhaconotus, Bathycentor, Ipodoryctes, Leptorhaconotus, Mimipodoryctes, Platyspathius, Rhaconotinus and some Rhaconotus). Third tergite usually without furrows, but strongly curved furrow known in Grangerdoryctes. Subbasal (in many cases) or submedial (Grangerdoryctes) transverse furrow present on fourth and fifth or sixth tergites. Fifth (Euryphrymnus, Pseudorhaconotus, Rhaconotus) or sixth (Arhaconotus, many Ipodoryctes, Mimipodoryctes, etc.) tergites more or less distinctly enlarged, often longer than previous ones; rarely these tergites in posterior margin with distinct submedian processes or median excision. Ovipositor (except Leptorhaconotus) relatively long, dorsally with double small preapical notches and apico-ventrally serrate.

\section{Key to the world genera of the tribe Rhaconotini}

1. Metasomal tergites with large lateral part strongly bent downwards (Fig. 23H); ventral margins of sixth tergite below almost connected or closely situated (Fig. 23F); metasoma tube-shaped in dissection. Acrosternite of first metasomal segment elongated, about 0.35 times as long as first tergite (Fig. 3H), its apical margin situated behind level of spiracles. Ovipositor basally flat and wide, evenly narrowed toward apex, strong upcurved, without dorsoapical tubercles and ventroapical serration (Figs 22K, 23C, E-G). Ovipositor sheath-wide and flat, leaf-shaped (Figs 22K, 23E, G). (Subtribe Leptorhaconotina)

Leptorhaconotus Granger, 1949

- Metasomal tergites with short lateral part slightly bent downwards (Figs 2D, 4C, 7D); ventral margins of sixth tergite below widely separated from each other; metasoma dorso-ventrally flattened (Figs 2D, 4C, 7D). Acrosternite of first metasomal segment usually not elongated, $0.20-0.25$ times as long as first tergite, its apical margin situated before level of spiracles (Figs 3K, 7D, 9B, 13D); occasionally acrosternite distinctly elongated (Antespathius and Platyspathius) (Figs 1J, 28L, 30J, 32I). Ovipositor round basally (in dissection), not narrowed toward apex and not upcurved, with dorsoapical tubercles and ventroapical serration (Figs 1A, 3A, 6A, 17D). Ovipositor sheath narrow and thick, stick-shaped (Figs 1A, 3A, 6A, 17D) (Subtribe Rhaconotina)

2. Second radiomedial vein $(\mathrm{r}-\mathrm{m})$ of fore wing absent (Figs 13A, 39A) ......................................... 3

- Second radiomedial vein $(\mathrm{r}-\mathrm{m})$ of fore wing present (Figs 2A, 4A, 5H, 9A) .................................. 4

3. First flagellar segment of antenna shorter than second segment (Fig. 12E). Third-fifth metasomal tergites with deep, strongly curved and distinctly crenulate furrows (Figs 13B-C). Second suture absent (Fig. 12I). Brachial (subdiscal) cell closed apically distinctly before recurrent vein (m-cu) (Fig. 13A) Grangerdoryctes Belokobylskij, 2005

- First flagellar segment of antenna longer than second segment (Fig. 38E). Third-fifth metasomal tergites without curved crenulate furrows (Fig. 39B). Second suture present and deep (Fig. 39B). Brachial (subdiscal) cell closed apically on the level of recurrent vein (m-cu) (Fig. 39A) 
4. Metasoma dorsally with only five visible tergites (following tergites hidden under fifth one); fifth tergite usually longer than previous tergite (Figs 9B, 11B, 45C, 46H, 48E)

- Metasoma dorsally with more than five (with sixth and seventh) visible tergites; sixth or seventh tergites longer or often not longer than previous tergite (Figs 2C, 4B, 5G, 7C, 15B)

5. Submedial (subbasal) cell of hind wing long; first abscissa of mediocubital vein $(\mathrm{M}+\mathrm{CU})$ 0.8-1.0 times as long as second abscissa (1-M) (Fig. 27B). Propodeum with elongate areola distinctly delineated by carinae (Fig. 26C). Fourth-fifth metasomal tergites almost entirely smooth (Figs 26C, 27C)

Neorhaconotus Belokobylskij, Iqbal \& Austin, 2004

- Submedial (subbasal) cell of hind wing short; first abscissa of mediocubital vein (M+CU) $0.3-0.5$ (0.6) times as long as second abscissa (1-M) (Figs 9A, 45B, 48A). Propodeum usually without areola delineated by carinae (Figs 8F, 44H, 46F, 47H). Fourth-fifth metasomal tergites usually mainly sculptured (Figs 9C, 45C, 46H, 48E)

6. Second metasomal tergite with basal area delineated posteriorly by distinct furrow (Figs 9C, 10H, 11B). Apical lenticular area of second tergite always present and sharply delineated anteriorly by deep and crenulate curved additional sulcus and posteriorly by deep second suture (Figs 9C, 10H, 11B)

Euryphrymnus Cameron, 1910

- Second metasomal tergite without basal area delineated posteriorly by furrow (Figs 45C, 46H, 48EF). Apical lenticular area of second tergite present or absent (Figs 45C, 46H, 48E-F) (Rhaconotus Ruthe, 1854)

7. Recurrent vein (m-cu) of fore wing distinctly antefurcal (Fig. 48A). Propodeum with distinct lateral tubercles (Fig. 47I). Apical segments of antenna contrasting pale (Fig. 47F) Subgenus Pararhacon Belokobylskij, 2005

- Recurrent vein (m-cu) of fore wing always postfurcal (Fig. 45A). Propodeum without distinct lateral tubercles (Fig. 44I). Apical segments of antenna same colour as previous segments (Fig. 44G) Subgenus Rhaconotus s. str.

8. First metasomal tergite narrow and long, weakly widened towards apex, petiolate or subpetiolate (Figs 1I-J, 2C-D, 30J, 31C-D, 35B, D, 37C-D); acrosternite of first segment more or less strongly or distinctly elongated (Figs 1J, 2D, 30J, 31D, 35D, 37D)

- First metasomal tergite rather wide and usually short, distinctly widened towards apex (Figs 4B-C, 5G, 7C-D, 15B-C, 17B, D), never petiolate; acrosternite of first segment not elongated, short (Figs $4 \mathrm{C}, 7 \mathrm{D}, 15 \mathrm{C}, 17 \mathrm{D})$ ...11

9. Face with dense and fine transverse aciculation (Fig.1C). Nervulus (cu-a) of fore wing distinctly antefurcal. First radiomedial vein (2-SR) absent (Figs 2A-B). Acrosternite of first metasomal segment long, 0.6-0.7 times as long as tergite (Figs 1J, 2D). First tergite distinctly striate with reticulation (Figs 1I, 2C) Antespathius Belokobylskij, 1995

- Face without dense transverse aciculation (Figs 28C, 30C, 32B, 34C, 36B). Nervulus (cu-a) of fore wing almost never antefurcal. First radiomedial vein (2-SR) always present (Figs 29A, 31A, 33A, 35A, 37A). Acrosternite of first metasomal segment rather short, 0.3-0.5 times as long as tergite (Figs 28L, 29C, 30J, 31D, 32I, 33D, 35D, 37D). First tergite usually densely and small areolate (Figs 28I, 29B, 31C, 32H, 33C, 34F, 35B, 37C). (Platyspathius Viereck, 1911) 10 
10. Vertex entirely densely granulate or reticulate-coriaceous, without or with sparse and incomplete striation (Figs 28D, 30D, 32C, 34D). Median lobe of mesonotum not high and usually weakly evenly-curved (Figs 28H, 30G, 32G, 34H). Second radiomedial (submarginal) cell of fore wing medium length, rather narrow, usually not widened apically (Figs 29A, 31A, 33A, $35 \mathrm{~A})$ Subgenus Platyspathius s. str.

- Vertex entirely densely and distinctly transverse striate (Fig. 36C). Median lobe of mesonotum highly hump-shaped (Fig. 36H). Second radiomedial (submarginal) cell of fore wing very long, wide, distinctly widened towards apex (Fig. 37A)

Subgenus Lenticularia van Achterberg, 2003

11. First and second metasomal tergites of female immovably fused (Figs 3J, K, 4B-C)

- First and second metasomal tergites of female movably connected (Figs 5G, 7C-D, 15B, C, 17B, D, 41C-D, 43D-E, 50C, E, 59C-D)

12. Parallel vein (CU1a) of fore wing always interstitial (Fig. 4A). First and second tergites of male immovably fused. Vertex smooth or densely granulate, without striation (Fig. 4C)

Arhaconotus Belokobylskij, 2000

- Parallel vein (CU1a) of fore wing usually not interstitial, arising from anterior third of distal margin of brachial (subdiscal) cell (Fig. 25A). First and second tergites of male movably connected. Vertex striate or rugose, sometimes with granulation between rugae, never smooth or only granulate (Fig. 4C)

Mimipodoryctes Belokobylskij, 2000

13. Second tergite with basal area delineated by furrow or different type of sculpture 14

- Second tergite without delineated basal area 16

14. Basal area of second tergite long and delineated posteriorly by deep furrow (Figs 5G, 7C). Parallel vein (CU1a) of fore wing arising from posterior third or occasionally from the middle of vein (3CU1) closing distally brachial (subdiscal) cell (Figs 5H, 7A). Recurrent vein (m-cu) of fore wing antefurcal or interstitial (Figs 5H, 7A)

Bathycentor Saussure, 1892

- Basal area of second tergite short and delineated posteriorly usually by only different type of sculpture (Figs 15B, 17B, 19B, 21B). Parallel vein (CU1a) of fore wing arising behind middle of vein (3-CU1) closing distally brachial (subdiscal) cell (Figs 15A, 17A, 19A, 21A). Recurrent vein (m-cu) of fore wing postfurcal (Figs 15A, 17A, 19A, 21A (Ipodoryctes Granger, 1949))

15. Hind coxa without or with very small basoventral tubercle (Figs 16B, 18J, 20H). Pro- and often mesothorax almost entirely smooth (Figs 16C-D, 18H-I, 20F-G). Notauli smooth or almost smooth (Figs 16C, 18H, 20F) Subgenus Afroipodoryctes subgen. nov.

- Hind coxa with distinct basoventral tubercle (Fig. 14H). Pro- and mesothorax at least partly granulate (Fig. 14F-G). Notauli crenulate or granulate (Fig. 14F) Subgenus Ipodoryctes s. str.

16. Recurrent vein (m-cu) antefurcal (Figs 50A, 51I, 52K, 53A, 55A, 57A). Parallel vein (CU1a) of fore wing not interstitial, arising behind middle of vein (3-CU1) closing distally brachial (subdiscal) cell (Figs 50A, 51I, 52K, 53A, 55A, 57A). First flagellar segment often not longer than second segment (Figs 49D, 51E, 52E, 54D, 56D). Metasoma behind third tergite smooth (Figs 50C-D, 51H, 53B-C, $55 \mathrm{~B}-\mathrm{C}, 57 \mathrm{~B}-\mathrm{C})$ Rhacontsira Belokobylskij, 1998

- Recurrent vein (m-cu) postfurcal (Figs 41A, 43A, 59A). Parallel vein (CU1a) of fore wing often interstitial (Figs 41A, 43A, 59A). First flagellar segment usually longer than second segment (Figs 40D, 42E, 58E). Metasoma behind third tergite sculpture at least in basal part of posterior tergites (Figs 41C, 43D, 59C) 
17. First metasomal tergite very long, 2.0-2.7 times as long as its apical width (Figs 58K, 59C-D). Sixth metasomal tergite distinctly enlarged and covered following apical tergites (Figs 59C-D). Mesosoma long, 2.5-2.7 times as long as maximum width (Figs 58I-J)

Troporhaconotus gen. nov.

- First metasomal tergite short, usually distinctly less than 2.0 times as long as its apical width (Figs 40G, 41C, 43C, E). Sixth metasomal tergite usually not enlarged and not covered following tergites (Figs 41C-D). Mesosoma short, 1.8-2.2 times as long as maximum width (Figs 40F, 42H) (Rhaconotinus Hedqvist, 1965)

18. Apical lenticular area of second metasomal tergite always present and sharply delineated anteriorly by deep and crenulate curved additional sulcus and posteriorly by deep second suture (Figs 40G, 41C) Subgenus Rhaconotinus s. str.

- Apical area of second metasomal tergite always absent; anteriorly additional transverse sulcus absent or almost indistinct (Fig. 43D) Subgenus Hexarhaconotinus subgen. nov.

Genus Antespathius Belokobylskij, 1995

Spathius (Antespathius) Belokobylskij, 1995: 49.

Spathius (Antespathius) - Long \& Belokobylskij 2011: 24.

Antespathius - Zaldívar-Riverón et al 2008: 358 (as genus in Rhaconotini). — Jasso-Martínez et al. 2019: 165.

\section{Type species}

Spathius (Antespathius) buonluoicus Belokobylskij, 1995, by original designation.

\section{Description (Figs 1-2)}

HEAD. Head not depressed, high, weakly transverse, face with very dense, even and slender transverse aciculation as on CD disc. Ocelli arranged in triangle with base larger than its sides. Frons flat, without median carina, with shallow longitudinal furrow. Antennal socket situated weakly lower than upper level of eyes (front view). Eyes glabrous. Occipital carina distinct, complete dorsally, ventrally obliterated at short distance of upper base of mandible. Malar suture very shallow, almost indistinct. Clypeus very narrow, with short lower flange. Hypoclypeal depression medium-sized, suboval. Postgenal bridge narrow. Maxillary palps long, 6-segmented, sixth (apical) segment 1.3 times as long as fourth segment; labial palps short, 4-segmented, second segment thickened, third segment weakly shortened, about 0.7 times as long as fourth segment. Scapus of antenna rather narrow and long, without apical lobe and basal constriction, its ventral margin (lateral view) shorter than dorsal margin, apically with distinct excavation in outer side. First flagellar segment subcylindrical, weakly curved, longer than second segment. Apical segment obtuse and without apical "spine".

Mesosoma. Mesosoma not depressed. Neck of prothorax rather long, without pronope. Pronotum dorsally flat (lateral view); pronotal carina fine and medially fused with posterior margin of pronotum at short distance. Propleural lateroposterior flange short and wide. Mesonotum highly and roundly elevated above pronotum. Median lobe of mesonotum weakly protruding forwards (lateral view), without median longitudinal furrow and anterolateral corners (dorsal view). Notauli complete, crenulate at least in anterior half, deep in anterior half and shallow in posterior half. Scuto-scutellar suture present and distinct. Prescutellar depression evenly long, rather deep, with several distinct carinae. Lateral longitudinal flanges on the level of prescutellar depression lower. Scutellum convex, with distinct lateral carinae. Metanotum medially without median carina and with short high lateral carinae (dorsal view); with short wide tooth (lateral view). Mesopleural pit transformed in narrow, long and oblique furrow 
running to median part of mesopleuron. Sternaulus (precoxal furrow) deep, wide, short. Prepectal carina complete, wide ventrally. Postpectal carinae absent. Metapleural flange short, wide, rounded apically. Propodeum without areas delineated by carinae, without depressions and lateral tubercles; propodeal bridge absent. Propodeal spiracles small and round, situated in basal 0.4 of propodeum.

WiNGs. Pterostigma of fore wing narrow and long. Radial vein (r) arising from middle of pterostigma. Radial (marginal) cell not shortened. First radiomedial vein (2-SR) absent, second radiomedial vein $(\mathrm{r}-\mathrm{m})$ present and sclerotised. Nervulus (cu-a) present and strongly antefurcal. Discoidal (discal) cell petiolate anteriorly, petiole (1-SR) short. Parallel vein (CU1a) interstitial. Mediocubital vein (M+CU1) distinctly sinuate. Brachial (subdiscal) cell closed postero-apically by evenly curved brachial vein (CU1b). Transverse anal veins (2A, a) absent. Hind wing with three hamuli. First abscissa of costal vein $(\mathrm{C}+\mathrm{SC}+\mathrm{R}) 0.8$ times as long as second abscissa $(1-\mathrm{SC}+\mathrm{R})$; first abscissa $(\mathrm{C}+\mathrm{SC}+\mathrm{R})$ not divided apically on two abscissae. Radial vein (SR) almost indistinct, arising from costal vein (2-SC+R) far from basal vein (1r-m). Radial (marginal) cell weakly and evenly widened toward apex, without additional transverse vein (r). Medial (basal) cell narrow, weakly widened towards apex from its base. Nervellus (cu-a) present and declivous. Submedial (subbasal) cell short. First abscissa of mediocubital vein $(\mathrm{M}+\mathrm{CU}) 0.45$ times as long as second abscissa (1-M). Recurrent vein (m-cu) rather short, strongly oblique toward base of wing, straight, hyaline.

LeGs. Fore tibia with several short and rather thick spines situated almost in single line. All tibiae slender. Middle tarsal segments rather long. Hind coxa rather short, wide, without basoventral corner and tubercle. Fore and middle femora with very low and wide dorsal protuberance. Hind femur wide, elongate-oval, without dorsal protuberance. Hind basitarsus short, about 0.6 times as long as secondfifth segments combined.

Metasoma. First metasomal tergite petiolate, long and narrow. Acrosternite of first segment 0.7 times as long as first tergite, its apical margin situated significantly behind spiracles. Dorsope of first tergite small; basolateral lobes absent. Spiracular tubercles present, distinct, situated in basal 0.3 of tergite; dorsal carinae distinctly only basally. Second and third tergites without any furrows and areas, its lateral parts fused. Second suture very shallow. Only second tergite with separate laterotergites. Fourth-sixth tergites with single submedian transverse line of short, sparse and semi-erect pale setae. Fifth tergite not enlarged, 0.9 times as long as fourth tergite; apical segments weakly protruding behind fifth tergite. Hypopygium in apical margin obtuse and rounded apically. Ovipositor sheath weakly widened towards subapex, not longer than metasoma.

\section{Diagnosis}

Antespathius was originally described as a subgenus of Spathius within the tribe Spathiini (Belokobylskij 1995). However, recent molecular phylogenetic studies that have included this taxon (Zaldívar-Riverón et al. 2008; Jasso-Martínez et al. 2019) have confirmed its placement within the tribe Rhaconotini (sensu nova) as a separate genus. Antespathius distinctly differs from the remaining Rhaconotini genera by having a considerable petiolate first metasomal tergite with strongly elongated acrosternite, equal to $0.6-0.7$ times length of tergite, face with very dense, even and slender transverse aciculation (as on CD disc), and nervulus (cu-a) of fore wing distinctly antefurcal. This genus distinctly differs from the Spathius species by the antefurcal position of nervulus (cu-a) in fore wing together with the loss of first radiomedial (2-SR) vein.

\section{Composition}

Antespathius buonluoicus (Belokobylskij, 1995) (OR), and an undescribed species from Madagascar (Zaldívar-Riverón et al. 2008). 


\section{Hosts}

Unknown.

\section{Distribution}

Oriental and Afrotropical regions.

\section{Remarks}

This genus remains monotypic with its single described species from Vietnam. However, a second, undescribed species of this genus has been recorded from Madagascar (Zaldívar-Riverón et al. 2008: 347).

Antespathius buonluoicus (Belokobylskij, 1995) stat. nov.

Figs 1-2

Spathius (Antespathius) buonluoicus Belokobylskij, 1995: 49.

Spathius (Antespathius) buonluoicus - Yu et al. 2016.

\section{Material examined}

\section{Holotype}

VIETNAM • +; "Vietnam, prov. GaiLai - ConTum, 20 km N Buon-Luoi, Tram Lap, 1-14.XII.1988, A. Sharkov"; ZISP.

\section{Paratypes}

VIETNAM • 10 우, 3 ふぇં; same collection data as for holotype but 30 Nov., 1-14 Dec. 1988; ZISP.

\section{Description}

\section{Female}

Measurements. Body length 2.6-3.3 mm; fore wing length 1.9-2.2 mm.

HEAD. Width 1.3-1.4 times its median length, 1.3 times width of mesoscutum. Head behind eyes (dorsal view) roundly narrowed. Transverse diameter of eye 1.3-1.5 times as long as temple. Ocelli small, arranged in triangle with base 1.2 times as wide as its sides. POL 1.3-1.7 times OD, 0.3-0.5 times OOL. Eyes almost without emargination opposite antennal sockets, weakly directed forwards, 1.2-1.3 times as high as broad. Malar space 0.6 times height of eye, 1.0-1.2 times basal width of mandible. Face distinctly convex, its width 1.1-1.25 times eye height, almost equal to height of face and clypeus combined. Hypoclypeal depression width $0.7-0.8$ times distance from edge of depression to eye, 0.4 times width of face. Tentorial pits distinct. Head below eyes (front view) roundly narrowed. Hypostomal flange rather wide. Maxillary palps about 1.1 times height of head (without mandibles). Antennae slender, almost filiform, 25-28-segmented. Scapus 1.8 times as long as its maximum width. First flagellar segment 4.5-5.3 times as long as its apical width, 1.25 times as long as second segment. Penultimate segment 2.7-3.1 times as long as wide, nearly as long as apical segment.

Mesosoma. Mesosoma elongated, its length 2.1 times maximum height. Maximum width of mesoscutum 1.1 times its length. Median lobe of mesoscutum protruding forwards, distinctly convex anteriorly (dorsal view). Prescutellar depression long, rather deep, with five distinct carinae, finely reticulate between carinae, medially 0.3 times as long as scutellum. Scutellum weakly convex, 1.2 times as long as its maximum width. Subalar depression entirely longitudinally striate with dense reticulation between striae. Propodeum (lateral view) distinctly and weakly curvedly inclined from base to apex. 


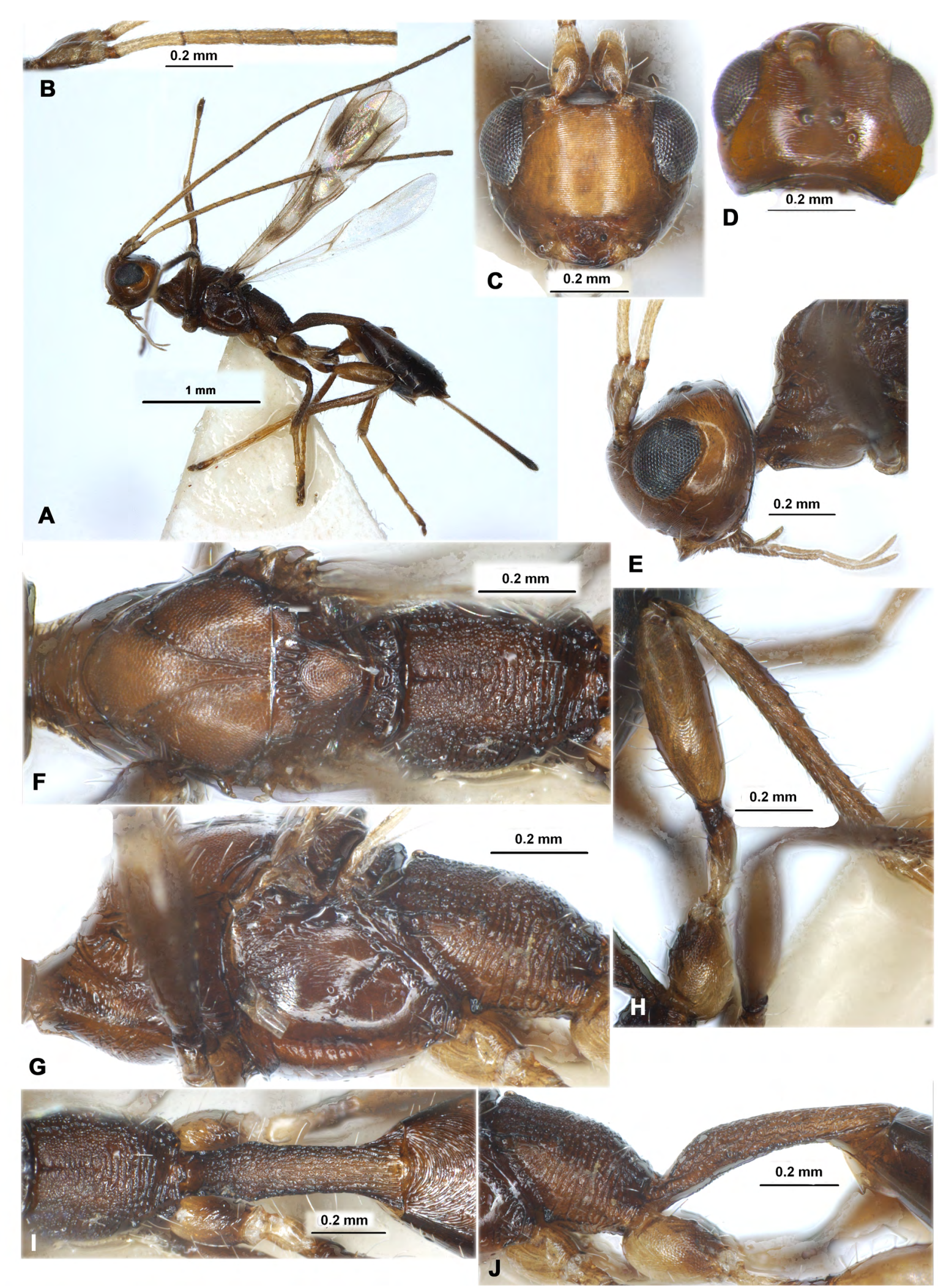

Fig. 1. Antespathius buonluoicus (Belokobylskij, 1995), , holotype, ZISP. A. Habitus, lateral view. B. Basal segments of antenna. C. Head, front view. D. Head, dorsal view. E. Head, lateral view. F. Mesosoma, dorsal view. G. Mesosoma, lateral view. H. Hind leg. I. Propodeum and petiole, dorsal view. J. Propodeum and petiole, lateral view. 


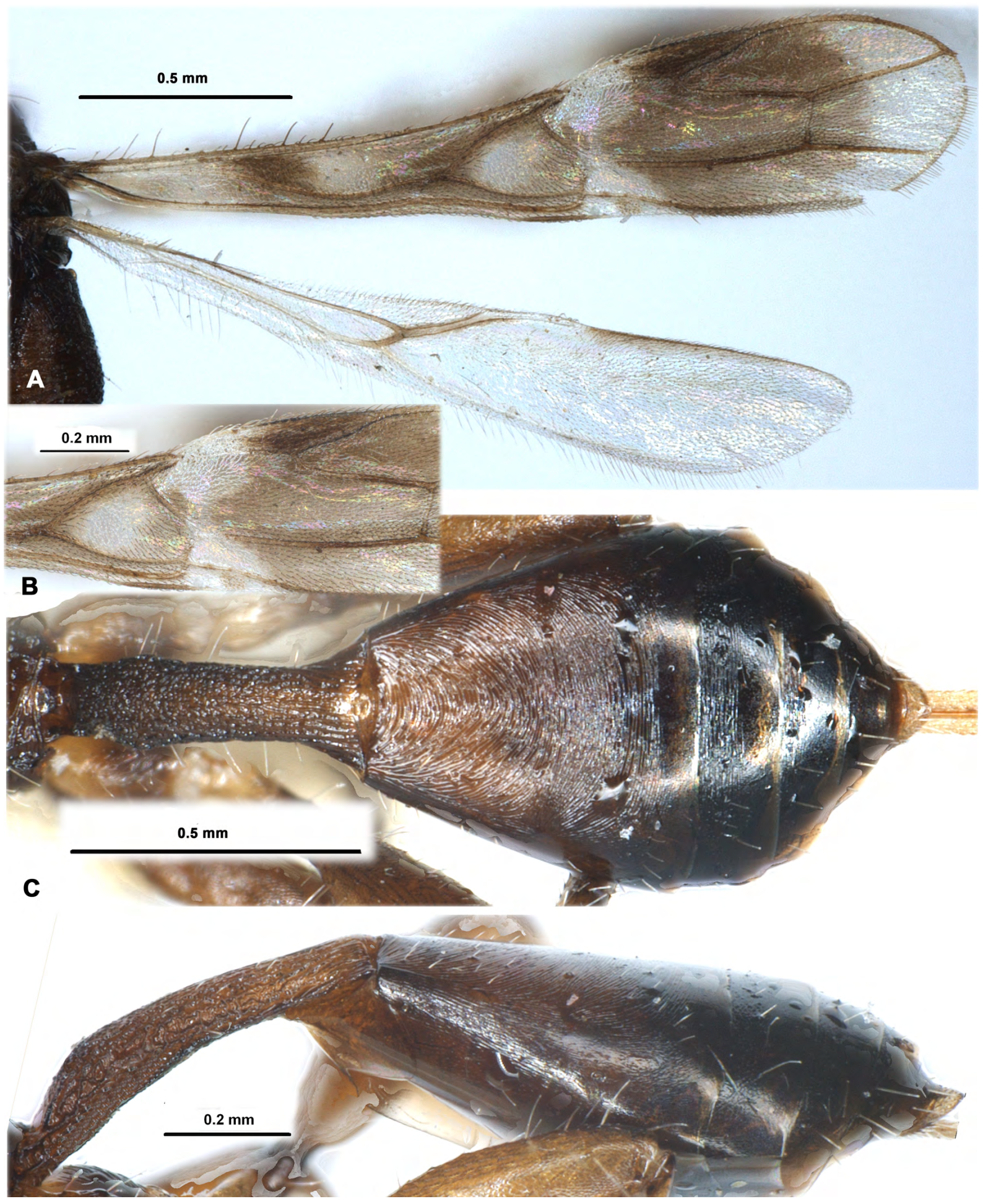

Fig. 2. Antespathius buonluoicus (Belokobylskij, 1995), + , holotype, ZISP. A. Wings. B. Fore wing, median part. C. Metasoma, dorsal view. D. Metasoma, lateral view. 
WiNGs. Fore wing 4.5-5.0 times its maximum width. Pterostigma 5.5 times as long as wide. Metacarpus (1-R1) 0.9-1.0 times as long as pterostigma. First radial abscissa (r) inclivous to pterostigma, 0.5 times as long as maximum width of pterostigma, forming obtuse angle with second radial abscissa (3SR). Second radial abscissa (3-SR) 6.0-7.4 times as long as first abscissa (r), 0.9-1.0 times as long as straight third abscissa (SR1), about as long as track of first radiomedial vein (2-SR). Second radiomedial (submarginal) cell (between recurrent ( $\mathrm{m}-\mathrm{cu}$ ) and second radiomedial $(\mathrm{r}-\mathrm{m})$ veins) long and wide, about 4.0 times as long as its maximum width, 1.3 times as long as the wide brachial (subdiscal) cell. First medial abscissa (1-SR+M) weakly sinuate. Discoidal (discal) cell wide and short, 1.5-1.8 times as long as wide. Basal (1-M) and recurrent (m-cu) veins divergent posteriorly. Distance from nervulus (cu-a) to basal (1-M) vein $0.5-1.5$ times nervulus (cu-a) length, nervulus (cu-a) straight and distinctly oblique towards base of wing. Hind wing 6.0 times as long as wide. Medial (basal) cell 14.8 times as long as its maximum width, $0.40-0.45$ times as long as wing. Radial (SR) and medial (2-M) veins almost indistinct. First abscissa of mediocubital vein $(\mathrm{M}+\mathrm{CU}) 0.4$ times as long as second abscissa (1-M). Recurrent vein (m-cu) strongly desclerotised, weakly curved, strongly antefurcal.

LeGS. Hind coxa 1.8 times as long as maximum width, 0.7 times as long as propodeum. Hind femur 3.03.1 times as long as wide. Hind tibia slender. Hind tarsus nearly as long as hind tibia. Hind basitarsus weakly thickened, without distinct ventral keel. Second segment of hind tarsus 0.5 times as long as first segment, 1.1-1.2 times as long as fifth segment (without pretarsus).

Metasoma. Weakly longer than head and mesosoma combined. Petiole dorsally convex in basal half and almost linear in apical half (lateral view), weakly widened on level of spiracles and apically (dorsal view), without convex median area. Apical width of petiole 1.2-1.5 times its width at level of spiracles, 1.6-2.0 times its minimum basal width; length of tergite 2.7-3.0 times its apical width, 1.4-1.5 times length of propodeum, $0.65-0.70$ times length of other tergites combined. Median length of second and third tergites combined 2.0-2.1 times basal width of second tergite, 0.75-0.9 times their maximum width. Ovipositor sheath $1.5-1.6$ times as long as petiole, 0.6 times as long as metasoma, 0.85 times as long as mesosoma, $0.4-0.5$ times as long as fore wing.

SCULPTURE AND PUBESCENCE. Vertex finely transversely striate-reticulate to coriaceous; frons entirely densely, sinuate aciculate; face as in generic description; temple almost smooth in anterior half and finely vertically striate in posterior half, almost smooth below. Mesoscutum densely and finely granulate-reticulate, with two distinct and weakly convergent posteriorly carinae in its medioposterior half. Scutellum finely and densely areolate-granulate. Mesopleuron mainly smooth, with fine striation anteriorly and rugulosity posteriorly. Metapleuron vertically striate with dense reticulation between striae. Propodeum rugose-granulate in basal 0.4 and transverse striate with reticulation between striae in posterior 0.6 , without areas, with medial carina in basal third. Hind coxa transverse striate dorsally, densely granulate-coriaceous to finely coriaceous laterally. Hind femur densely and finely granulate-coriaceous. Petiole densely undulately striate with dense rugulosity between striae. Second and third tergites very densely concentrically aciculate, third tergite smooth apically, fourth and fifth tergites densely and finely transversely aciculate in basal halves and smooth ion apical halves. Vertex mainly glabrous, with sparse, long and semi-erect pale setae laterally and posteriorly. Mesoscutum mainly glabrous, with very sparse, semi-erect and long pale setae arranged along notauli and laterally. Metapleuron mainly glabrous. Hind tibia dorsally with rather long, sparse, semi-erect, pale setae, their length 0.5-1.0 times maximum width of tibia.

CoLour. Head reddish brown to light reddish brown. Mesosoma reddish brown to partly dark reddish brown. Petiole light reddish brown, second metasomal tergite dark reddish brown, remaining metasoma almost black. Antennae yellow to light brown in basal half, scapus brown dorsally, brown to dark brown in apical half. Palps reddish brown basally to yellow apically. Legs reddish brown to dark reddish 
brown, all coxae and tarsi (excluding fifth segments) and hind femur light reddish brown to brownish yellow; middle and hind tibiae subbasally yellow. Fore wing distinctly maculate, with brown spots nearly middle of medial (basal) cell, around basal (1-M) and recurrent (m-cu) veins and around second radiomedial (submarginal) cell. Pterostigma dark brown, pale yellow in basal $1 / 3$ or $2 / 5$.

\section{Male}

MEASUREMENTS. Body Length 2.6-2.8 mm; fore wing length $1.7-2.0 \mathrm{~mm}$.

Metasoma. First metasomal tergite slender, 3.6 times as long as apical width, 1.5-1.6 times as long as propodeum.

Otherwise, similar to female.

\section{Distribution}

Vietnam.

Genus Arhaconotus Belokobylskij, 2000

Arhaconotus Belokobylskij, 2000: 345.

Arhaconotus - Belokobylskij 2001: 157.- Belokobylskij et al. 2004: 22. - Belokobylskij \& Maeto 2009: 59. — Tang et al. 2010: 63. - Yu et al. 2016. — Jasso-Martínez et al. 2019: 165.

\section{Type species}

Arhaconotus papuanus Belokobylskij, 2000, by original designation.

\section{Description (Figs 3-4)}

HEAD. Head not depressed, high, transverse. Ocelli arranged almost in equilateral triangle. Frons not concave, without median keel or furrow. Eyes glabrous. Occipital carina complete dorsally, joined ventrally below with hypostomal carina. Malar suture fine. Clypeal suture distinct and complete. Clypeus with short ventral flange. Hypoclypeal depression medium-sized, round. Postgenal bridge narrow. Palps long; maxillary palps 6-segmented, its fifth segment longest, 1.4 times as long as sixth (apical) segment; labial palps 4-segmented, its third segment not shortened. Scapus wide and short, without apical lobe and basal constriction; its ventral margin (lateral view) not longer than dorsal margin. First flagellar segment subcylindrical, almost straight, weakly longer than second segment.

Mesosoma. Mesosoma not depressed and relatively long. Neck of prothorax short. Pronotum dorsally almost flat, with distinct submedian pronotal carina. Pronope absent. Propleural dorsoposterior flange rather short and not wide. Mesonotum high, gently and roundly elevated above pronotum, mainly smooth. Median lobe of mesonotum with median longitudinal furrow in posterior half, without anterolateral corner. Notauli entirely deep and complete. Tegula short, widened distally, weakly convex along its posterior margin. Prescutellar depression deep, long, with several carinae. Lateral longitudinal winglike flanges situated on level of prescutellar depression low. Scuto-scutellar suture rather distinct and complete. Scutellum weakly convex, without lateral carinae. Metanotum with short, but distinct and pointed median tooth posteriorly. Mesopleural pit deep, subround, connected with mesopleural suture. Sternaulus (precoxal sulcus) rather deep, not wide, long, running along whole length of lower part of mesopleuron, almost straight, smooth. Prepectal carina distinct and complete, high below opposite coxae, laterally following up till subalar furrow. Postpectal carina absent. Metapleural flange rather long, wide, round apically. Propodeum with distinctly delineated by carinae basolateral areas and areola; lateral tubercles and propodeal bridge absent. Propodeal spiracles small and round. 
Wings. Pterostigma of fore wing wide. Radial vein (r) arising almost from middle of pterostigma. Radial (marginal) cell not shortened. Both radiomedial veins (2-SR, r-m) present. Second radiomedial (submarginal) cell long and narrow. Recurrent vein (m-cu) weakly postfurcal. Discoidal (discal) cell petiolate anteriorly, petiole (1-SR) rather long. Nervulus (cu-a) postfurcal. Brachial (subdiscal) cell gently and almost linearly closed apically before recurrent vein (m-cu). Parallel vein (CU1a) interstitial. Transverse anal veins $(2 \mathrm{~A}$, a) absent. Hind wing with three hamuli. First abscissa of costal vein $(\mathrm{C}+\mathrm{SC}+\mathrm{R}) 0.4$ times as long as second abscissa $(1-\mathrm{SC}+\mathrm{R})$. Radial vein $(\mathrm{SR})$ arising from costal vein $(2-\mathrm{SC}+\mathrm{R})$ not far separating from basal vein $(1 \mathrm{r}-\mathrm{m})$. Radial (marginal) cell subparallel-sided, without transverse vein (r). Medial (basal) cell distinctly widened towards apex, about 9.0 times as long as its wide, 0.45 times as long as hind wing. Nervellus (cu-a) present. Submedial (subbasal) cell short. First abscissa of mediocubital vein (M+CU) 0.5-0.6 times as long as second abscissa (1-M). Recurrent vein (m-cu) short, antefurcal, strongly oblique towards base of wing, unclerotised.

LEGS. Fore and middle tibiae with dense short and thick spines arranged almost in single row. Fore tarsus 1.5 times as long as fore tibia. Middle tarsal segments rather short. Hind coxa rather long and wide, with distinct basoventral corner and tubercle. All femora with more or less distinct dorsal protuberances. Hind tibia with spine with single row of dense white setae in inner distal margin. Hind femur wide. Inner spur of hind tibia rather long, glabrous, but with row of setae on basal half, 0.2 times as long as hind basitarsus; outer spur short, about 0.5 times as long as inner spur. Hind basitarsus about 0.9 times as long as second-fifth segments combined. Claws short and simple.

Metasoma. Widened from base till end of fore tergite, then rather evenly narrowed to apex, with six visible tergites, but seventh tergite also weakly protruding behind sixth tergite. First metasomal tergite not petiolate, wide, immovably fused with second tergite in both sexes. Acrosternite of first segment about 0.2 times as long as first tergite, its apical margin placed distinctly before spiracles. Dorsope of first tergite small but distinct; present short and wide basolateral lobes; spiracular tubercles indistinct, spiracles placed in basal third of tergite; dorsal carinae complete and parallel. Second suture deep, regularly curved, without lateral bends. Second tergite with short semi-oval basal area, with long lenticular apical area delineated by deep concave furrow. Third tergite without transverse depression or furrow. Second-sixth tergites with separate laterotergites. Sixth tergite of female not enlarged, without transformations, not or only weakly longer than previous tergite, not entirely covered following apical segments. Hypopygium small, pointed medioposteriorly. Ovipositor almost as long as metasoma. Apical part of ovipositor with two small dorsal nodes and serrate ventrally.

\section{Diagnosis}

This genus is very similar to Bathycentor Saussure, 1892 but differs from it mainly immovably fused first and second metasomal tergites in both sexes (vs not fused), the interstitial position of parallel vein (CU1a) in fore wing (vs not interstitial) and postfurcal position of recurrent vein (m-cu) (vs antefurcal).

\section{Composition}

Arhaconotus hainanensis Tang \& Chen, 2010 (OR); A. ishigakiensis Belokobylskij, 2001 (OR); A. papuanus Belokobylskij, 2000 (AU); A. vietnamicus Belokobylskij, 2001 (OR).

\section{Hosts}

Unknown.

\section{Distribution}

Australasian and Oriental regions.

\section{Remark}

A key to all described species of Arhaconotus was published by Tang et al. (2010: 67). 
Arhaconotus papuanus Belokobylskij, 2000

Figs $3-4$

Arhaconotus papuanus Belokobylskij, 2000: 346.

Arhaconotus papuanus - Belokobylskij 2001: 162. — Yu et al. 2016. — Jasso-Martínez et al. 2019: 167.

\section{Material examined}

Holotype

PAPUA NEW GUINEA • 9 ; "Papua New Guinea, Milne Bay Prov., Cape Vogel, Menapi, Oct. 24-29, 1989, R. Wharton"; TAMU.

\section{Paratypes}

PAPUA NEW GUINEA • 1 \% ; same collection data as for holotype; TAMU $\bullet 1$ त ; same collection data as for holotype; ZISP.

\section{Description}

Female

MEASUREMENTs. Body length 3.7-4.3 mm; fore wing length $2.7-3.1 \mathrm{~mm}$.

HEAD. Head width about 1.5 times its median length, 1.35 times width of mesoscutum. Head behind eyes (dorsal view) weakly convex in anterior half, strongly and almost linearly narrowed in posterior half. Transverse diameter of eye 2.0-2.4 times as long as temple. Ocelli medium-sized, arranged almost in equilateral triangle; POL almost equal to OD, 0.4-0.5 times OOL. Eye distinctly emarginated opposite antennal sockets, 1.2-1.3 times as high as broad. Malar space 0.3 times height of eye, 0.6-0.7 times basal width of mandible. Face width $0.8-0.9$ times eye height and 1.2 times height of face and clypeus combined. Upper margin of clypeus situated distinctly higher than lower level of eyes. Width of hypoclypeal depression 0.8-1.0 times distance from depression to eye, 0.4-0.5 times width of face. Vertex convex. Head below eyes (front view) distinctly and almost linearly or weakly roundly narrowed. Antennae filiform, more then 24-segmented (apical segments missing). Scapus 1.5-1.6 times as long as its maximum width, 2.0 times length of pedicel. First flagellar segment 4.5-5.0 times as long as its apical width, 1.1 times as long as second segment.

Mesosoma. Length about 2.0 times its height. Pronotal carina distinct, situated submedially. Mesoscutum (dorsal view) about as long as wide. Notauli deep in anterior half and shallower in posterior half, rather wide, sparsely and finely crenulate. Median lobe of mesoscutum distinctly convex anteriorly. Prescutellar depression with high median and two lateral carinae, almost smooth on rest part, distinctly curved along posterior margin, 0.3 times as long as scutellum. Scutellum length almost equal to its maximum width. Subalar depression coarsely crenulate. Sternaulus (precoxal furrow) entirely smooth. Prepectal carina with low lobes opposite fore coxae. Metanotum dorso-medially with two high and curved carinae. Propodeum weakly arcuately sloping backward (lateral view). Metapleural flange posteriorly with dense pubescence.

WINGS. Length of fore wing 3.4-3.7 times its maximum width. Pterostigma 4.0-4.5 times as long as wide. Metacarpus (1-R1) 1.1-1.2 times as long as pterostigma. Radial (marginal) cell 3.3 times as long as wide. Radial vein (r) arising from middle of pterostigma. First radial abscissa (r) forming very obtuse corner with second abscissa (3-SR). Second radial abscissa (3-SR) 3.8 times as long as first abscissa (r), 0.75-0.80 times as long as the straight third abscissa (SR1), 1.6-1.8 times as long as first radiomedial vein (2-SR). Second radiomedial (submarginal) cell long, not widened distally, 3.2-3.5 times as long as wide, about 1.7 times as long as the narrow brachial (subdiscal) cell. First medial abscissa (1-SR+M) 


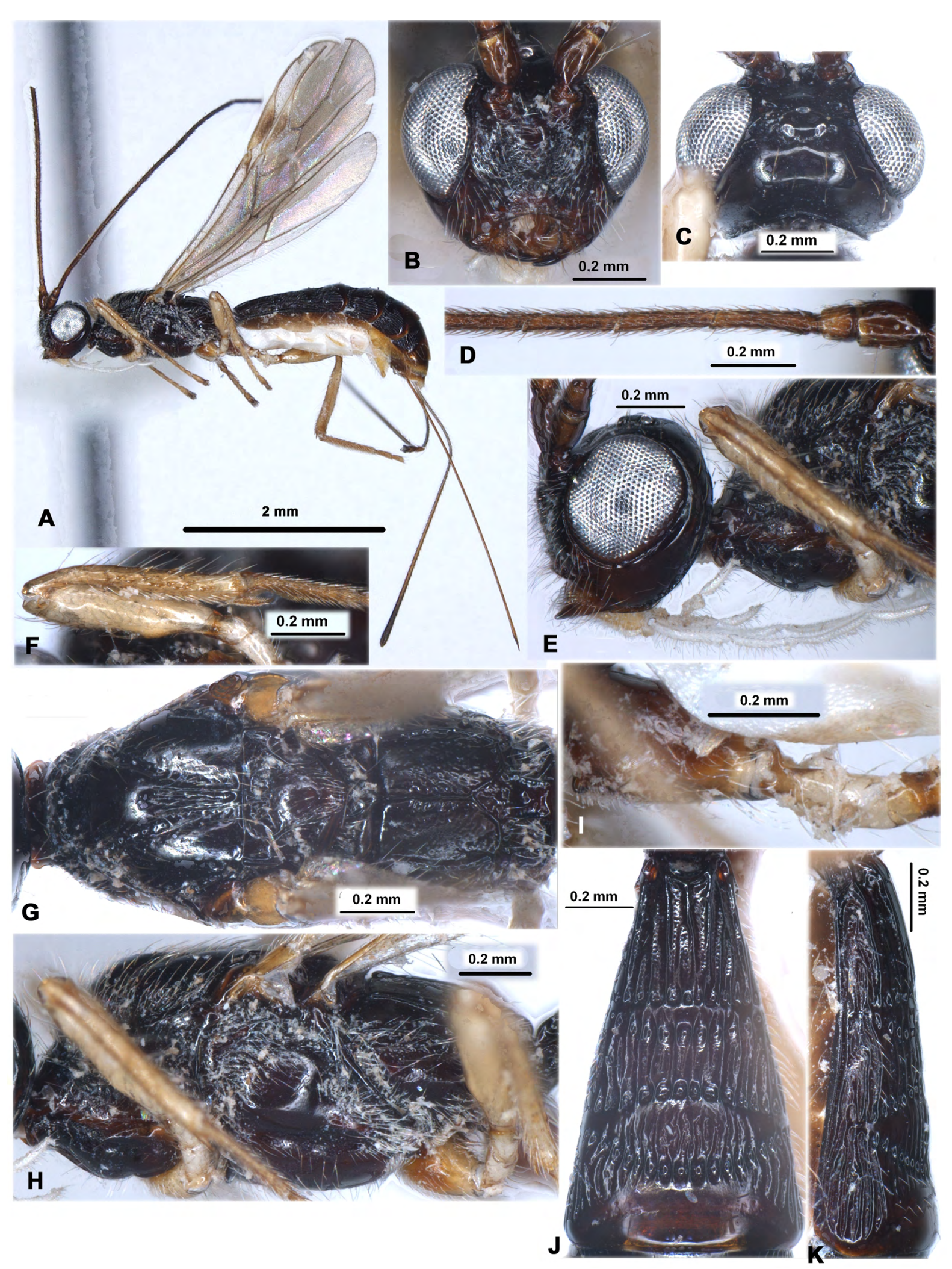

Fig. 3. Arhaconotus papuanus Belokobylskij, 2000, + , holotype, TAMU. A. Habitus, lateral view. B. Head, front view. C. Head, dorsal view. D. Basal segments of antenna. E. Head, lateral view. F. Fore leg. G. Mesosoma, dorsal view. H. Mesosoma, lateral view. I. Hind coxa. J. First to third metasomal tergites, dorsal view. K. First to third metasomal tergites, lateral view. 

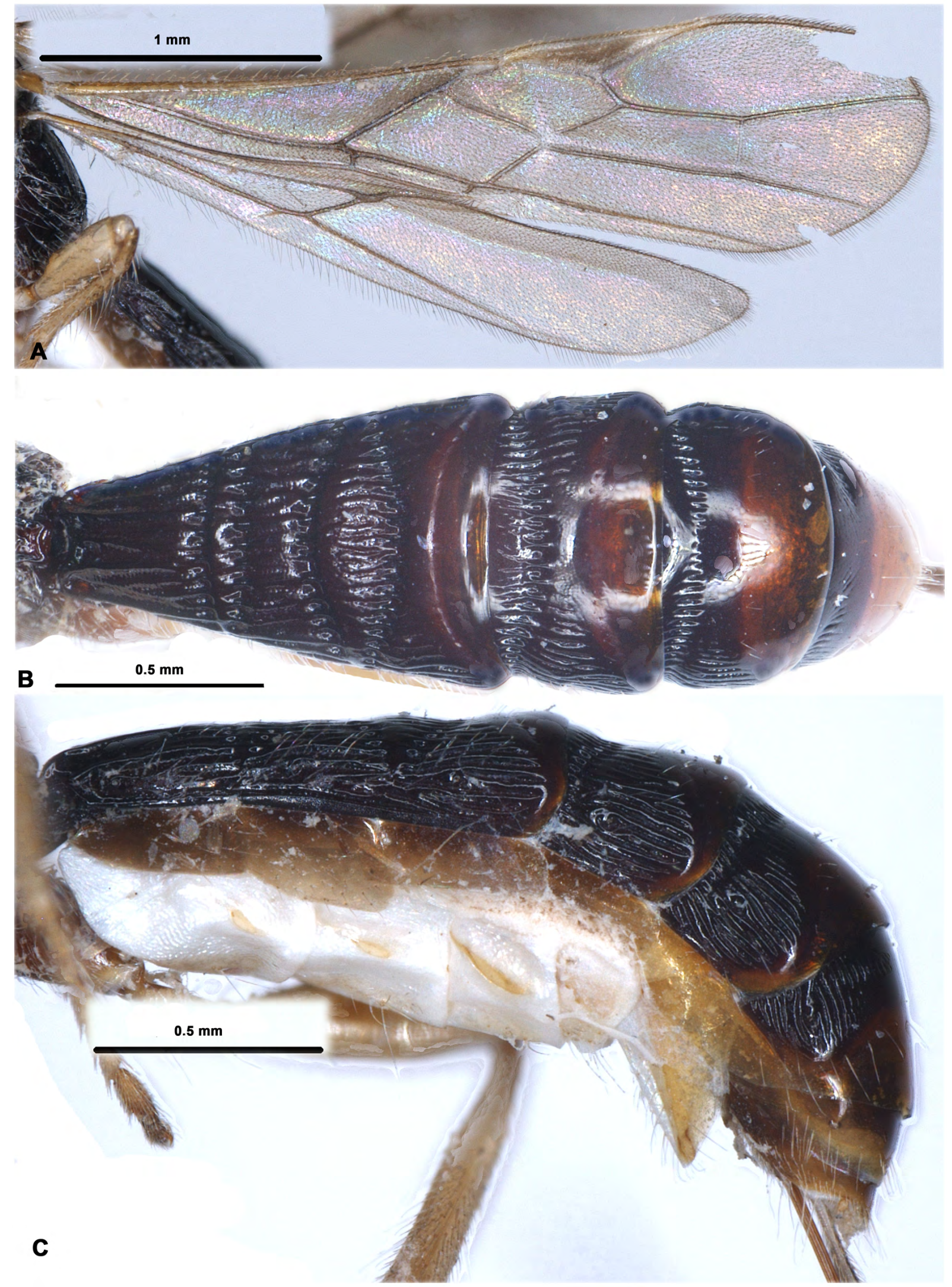

Fig. 4. Arhaconotus papuanus Belokobylskij, 2000, +, holotype, TAMU. A. Wings. B. Metasoma, dorsal view. C. Metasoma, lateral view. 
very weakly sinuate. Second abscissa of medial vein $(2-\mathrm{SR}+\mathrm{M})$ short or very short. Recurrent vein (m-cu) 0.8 times as long as first radiomedial vein (2-SR). Mediocubital vein (M+CU1) weakly curved in apical half. Distance from nervulus (cu-a) to basal vein (1-M) 0.5-0.7 times nervulus (cu-a) length. Brachial (subdiscal) cell closed distinctly before recurrent vein $(\mathrm{m}-\mathrm{cu})$; posterior bulla present but short; posterior abscissa of anal vein (2-1A) (behind brachial vein (CU1b)) present. Hind wing 6.0 times as long as wide. Recurrent vein (m-cu) straight, strongly oblique towards base of wing.

LeGs. Hind coxa almost 2.0 times as long as wide (with tooth). Hind femur 3.2 times as long as wide. Hind tarsus 1.0-1.1 times as long as hind tibia. Hind basitarsus with lower keel and with very dense pale setosity. Second segment of hind tarsus 0.3 times as long as basitarsus, 1.1-1.2 times as long as fifth segment (without pretarsus).

Mesosoma. Metasoma 1.4 times as long as head and mesosoma combined, with sixth visible tergites, remaining segments weakly protruding behind sixth tergite. First tergite distinctly, almost linearly and evenly widened from base to apex. Maximum width of tergite almost 2.0 times its minimum basal width; length $0.9-1.0$ times its apical width, 0.8 times length of propodeum. Apical area of second tergite 0.75 times as long as remaining of tergite. Median length of second tergite (with areas) 1.0-1.2 times its basal width, almost 2.0 times median length of third tergite. Sixth tergite weakly enlarged, almost straight on posterior margin, without median emargination and postero-ventral lobes. Sixth tergite almost as long as fifth and about 1.1 times as long as fourth tergites. Ovipositor sheath about 1.1 times as long as metasoma, about 2.0 times as long as mesosoma, 0.9 times as long as fore wing.

SCULPTURE AND PUBESCENCE. Head smooth, face rugulose-striate in rather narrow vertical stripes near smooth median and lateral areas. Mesonotum and mesopleuron smooth. Metapleuron smooth and with fine reticulation or granulation in anterior half, rugulose in posterior half. Propodeum with large, mainly coriaceous, with fine rugosity along carinae and almost smooth basally basolateral areas; areola rather small, rugulose, 1.5 times as long as maximum width; median carina 2.0-2.5 times as long as anterior fork of areola; propodeum in posterior third rugulose. Hind legs smooth. First and second tergites entirely (but second in apical area finely), third tergite in basal 0.2 medially and 0.8-0.9 laterally, fourth and fifth tergites in basal furrow only medially and in basal 0.8 laterally distinctly striate. Remaining parts of tergites and sixth tergite almost entirely smooth. Second-sixth tergites laterally coarsely and densely striate but smooth apically. Vertex glabrous medially, with sparse long and semi-erect setae posteriorly and laterally. Mesonotum with dense semi-erect and rather short setae arranged wide area along notauli and marginally. Mesopleuron mostly glabrous. Hind tibia dorsally with dense numerous short setae and long sparse semi-erect setae, long setae $0.6-0.8$ times as long as maximum width of tibia. Laterotergites shortly and rather sparsely setose.

Colour. Body black, head below and third-sixth tergites in apical halves reddish. Antenna reddish brown basally, darkened toward apex. Palps pale yellow. Legs brownish yellow, middle coxae basally, hind coxae entirely and base of hind tibia reddish brown, sometimes darker, rarely middle coxa light reddish brown; hind femur in basal half brownish. Ovipositor sheath medially light reddish brown or reddish brown, dark in basal third and apically. Fore wing distinctly and evenly infuscate. Pterostigma brown, yellow in basal basally and in apical $1 / 4$ or $1 / 3$.

\section{Male}

Measurements. Body length $2.9 \mathrm{~mm}$, fore wing length $2.1 \mathrm{~mm}$. Malar space 0.25 times height of eye and 0.55 times basal width of mandible.

WINGS. Second radial abscissa (3-SR) 5.3 times as long as first abscissa (r), 0.9 times as long as third abscissa. Second radiomedial (submarginal) cell 4.0 times as long as wide, 2 times as long as brachial (subdiscal) cell. 
LeGs. Hind femur 2.7 times as long as width. Second tarsal segment of hind leg 0.25 times as long as basitarsus, 0.9 times as long as fifth segment (without pretarsus).

Metasoma. Narrow. Length of first tergite 1.35 times its apical width. Apical area of second tergite 0.6 times as long as remaining part of tergite. Length of second tergite 1.6 times its basal width, 1.8 times length of third tergite. Basal and apical areas of second tergite almost entirely smooth. Third and fourth tergites coarse striate in basal 0.6-0.7; fifth and sixth tergites reticulate-coriaceous in basal 0.7-0.8. Apical tergites not concealed by sixth tergite.

CoLour. Body dark reddish brown. Hind femur almost entirely brownish. Otherwise similar to female.

\section{Distribution}

Papua New Guinea.

\section{Genus Bathycentor Saussure, 1862}

Bathycentor Saussure in Grandidier, 1892: pl. 21.

Epirhacon Belokobylskij, 1990b: 141 (type species: Epirhacon laetus Belokobylskij, 1990, by original designation).

Sinaodoryctes Chen \& Shi, 2004: 59 (type species: Sinaodoryctes aurus Chen \& Shi, 2004, by original designation).

Bathycentor - Viereck 1914: 20. — Shenefelt \& Marsh 1976 : 1267. — Yu at al. 2016. — Belokobylskij 2018: 506, 766. - Jasso-Martínez et al. 2019: 165.

Epirhacon - Belokobylskij 1992: 907 (as synonym of Ipodoryctes Granger, 1949); 2018: 506.

Sinaodoryctes - Belokobylskij 2018: 506.

\section{Type species}

Bathycentor kraesselini Saussure, 1892, by subsequent designation (Viereck 1914).

\section{Description (Figs 5-7)}

HEAD. Head transverse (dorsal view). Ocelli small or medium-sized, arranged in obtuse triangle. Frons weakly concave or almost flat, with or without small median furrow. Eyes glabrous. Occipital carina distinct, below reduced over wide or narrow distance. Malar suture usually absent, rarely developed but very shallow. Clypeal suture complete. Hypoclypeal depression rather large, round or widely oval. Face above clypeal suture with two distinct or inconspicuous oblong-oval submedian depressions. Palps rather long; maxillary palps 6-segmented; labial palps 4-segmented. Postgenal bridge developed but narrow. Scapus of antenna wide and rather long, without apical lobe. First flagellar segment longer than second segment.

Mesosoma. Mesosoma not depressed dorsoventrally. Neck of pronotum short, convex dorsally (lateral view). Pronotal keel more or less distinct, situated nearly middle of pronotum. Posterodorsal propleural lobe distinct and wide. Mesoscutum usually highly and roundly elevated above pronotum. Median lobe of mesoscutum clearly protruding forwards, without anterolateral corners, with rather distinct or indistinct and complete or incomplete median furrow. Notauli deep (at least in anterior half), wide, always complete, coarsely or clearly rugose or crenulate. Prescutellar depression rather long, relatively deep, more or less distinctly sculptured, with median carina. Scuto-scutellar suture developed. Scutellum convex, with or without fine lateral carinae. Metanotum without median tooth, occasionally with short and thick tooth (lateral view). Sternaulus (precoxal furrow) rather deep, narrow, more or less long. Prepectal carina distinct. Prepectus additionally with two distinct longitudinal lateral carinae. Postpectal 
carina absent. Metapleural lobe rather short, more or less wide, rounded at apex, with coarse median carina. Propodeum usually with areas delineated by rather distinct carinae; lateral tubercles absent or (occasionally) poorly developed; propodeal bridge narrow. Propodeal spiracle small.

WiNGS. Radial vein (r) of fore wing arising from, or slightly before middle of pterostigma. Radial (marginal) cell not shortened. First (2-SR) and second ( $\mathrm{r}-\mathrm{m})$ radiomedial veins present. Recurrent vein (m-cu) interstitial or antefurcal. Discoidal (discal) cell petiolate anteriorly. Mediocubital vein (M+CU1) in distal half more or less curved towards anal vein (1-1A). Nervulus (cu-a) distinctly postfurcal. Parallel vein (CU1a) non-interstitial, arising rarely from middle or, more frequently, behind middle (from posterior $0.3-0.4$ ) of vein (3-CU1) closing distally brachial (subdiscal) cell. Brachial (subdiscal) cell always closed distally by brachial vein (CU1b). Transverse anal veins (2A, a) absent. Hind wing with three hamuli. Nervellus (cu-a) developed. Submedial (subbasal) cell closed by veins and rather long. First mediocubital abscissa $(\mathrm{M}+\mathrm{CU})$ shorter than second abscissa (1-M). Recurrent vein (m-cu) long, sclerotized or pigmented. Medial (basal) cell wide, widened towards apex, about half as long as hind wing. Radial (marginal) cell subparallel-sided, without additional cross vein (r).

LEGS. Fore and middle tibiae with rather sparse and thick spines arranged almost in single line. Hind coxa medium-sized, with distinct basoventral tubercle. All femora with small and wide dorsal tubercles. Hind femur rather slender or more or less thickened. Inner spur of hind tibia rather long, $0.30-0.35$ times as long as hind basitarsus; outer spur short. Hind basitarsus with narrow but rather distinct carina on lower margin, 0.5-0.8 times as long as second-fifth segments of hind tarsus combined.

Metasoma. First tergite not petiolate. Its acrosternite about 0.2 times as long as tergite. Dorsope of first tergite distinct; basolateral lobes absent or only poorly developed; spiracular tubercles indistinct or poorly developed; dorsal carinae developed though occasionally present only at base of tergite. Second tergite with large semi-oval basal area separated along posterior margin by wide and rather deep, curved and crenulate furrow, with distinct wide and anteriorly curved or (rarely) nearly straight posterior furrow separating anteriorly wide lenticular apical area. Second suture distinct, rather deep, more or less wide. Fourth and (occasionally) fifth tergites with small, curved and crenulate basal furrow. Second-sixth tergites with separate laterotergites (epimera). Sixth tergite distinctly enlarged, as long as or longer than fifth tergite. Hypopygium rather short, pointed or slightly obtuse in middle of posterior margin.

\section{Diagnosis}

Bathycentor is morphologically very similar to Ipodoryctes, but it differs from the latter by having the following characters: recurrent vein (m-cu) of the fore wing antefurcal or interstitial (vs postfurcal); parallel vein (CU1a) of the fore wing arised from the posterior third or (less frequently) almost from the middle of the vein (3-CU1) closing the brachial (subdiscal) cell distally (vs before middle or sometimes interstitial); basal area of the second metasomal tergite long and separated by a deep furrow along the posterior margin (vs short and usually weakly separated often only different type of sculpture).

\section{Composition}

Bathycentor aurus (Chen \& Shi, 2004)(OR); B. kraesselini Saussure, 1892 (AF); B. laetus (Belokobylskij, 1990) (OR); B. parallelus (Granger, 1949) (AF); B. tricoloricornis (Granger, 1949) comb. nov. (AF); B. zimbabwensis sp. nov. (AF).

\section{Hosts}

Unknown. 


\section{Distribution}

Afrotropical and Oriental regions.

\section{Remarks}

The revision of this genus with designation of its lectotype, new synonymies and description of new species was recently published by Belokobylskij (2018).

\section{Key to species of Bathycentor}

1. Posterior margin of sixth metasomal tergite regularly convex, without median processes or emargination, usually without arcuate striae (Fig. 5G)

- Posterior margin of sixth metasomal tergite with median processes or emargination, with distinct arcuate striae (Fig. 7C)

2. Vertex rugose. Temples short; transverse diameter of eye (dorsal view) more than 2.0 times length of temple. Malar space 0.3 times as high as eye. Mesoscutum very weakly elevated above pronotum. Sternaulus crenulate. Mesosoma and metasoma mainly reddish-brown. Length of body $4.4 \mathrm{~mm}$. China

B. aurus (Chen \& Shi, 2004)

- Vertex punctate-granulate, without rugae (Fig. 5B). Temples long; transverse diameter of eye (dorsal view) 1.3-1.4 times length of temple. Malar space half as high as eye (Fig. 5C). Mesoscutum highly elevated above pronotum (Fig. 5F). Sternaulus coriaceous to finely granulate (Fig. 5F). Mesosoma and metasoma mainly dark brown to black (Fig. 5F)

3. Recurrent vein of fore wing antefurcal. All coxae, trochanters, trochantelli, and tibiae yellow or brownish yellow. Ovipositor sheath yellow except for black apex. Hind tibia distinctly thickened towards apex. Flagellum of antenna behind second segment yellow. Length of body $12.7 \mathrm{~mm}$. Madagascar

B. parallellus (Granger, 1949)

- Recurrent vein of fore wing almost interstitial (Fig. 5H). All coxae, trochanters, trochantelli, and tibiae nearly black (Fig. 5A). Ovipositor sheath nearly black in basal 0.4-0.5 and at apex, pale brown in subapical part (Fig. 5A). Hind tibia weakly thickened towards apex (Fig. 5A). Flagellum of antenna behind second segment reddish-brown (Fig. 5A). Length of body 12.1-15.0 mm. Madagascar

B. kraesselini Saussure, 1892

4. Posterior margin sixth metasomal tergite with two distinct median processes. Pterostigma entirely brown. Length of body $8.9 \mathrm{~mm}$. Democratic Republic of Congo

B. trjapitzini Belokobylskij, 2018

- Posterior margin of sixth metasomal tergite with median emargination, without distinct processes (Fig. 7C). Pterostigma dark brown, with pale areas at least at base (Fig. 7A)

5. Propodeum without areas delineated by carinae. Second tergite long, its length (with posterior area) along midline equal to its basal width. Basal (anterior) area of second tergite rather long medially and narrow anteriorly, along midline about 0.3 times length of tergite, its posterior area 0.5 times as long as tergite length. Length of body $5.7 \mathrm{~mm}$. Vietnam

B. laetus (Belokobylskij, 1990)

- Propodeum with areas delineated by distinct carinae (Fig. 6F). Second tergite short, its length (with posterior area) along midline 0.6 times its basal width (Fig. 7C). Basal (anterior) area of second tergite short medially and wide anteriorly, along midline 0.15 times length of tergite, and posterior its area 0.7 times as long as tergite length (Fig. 7C). Length of body $3.5 \mathrm{~mm}$. Zimbabwe B. zimbabwensis sp. nov. 
Bathycentor kraesselini Saussure, 1892

Fig. 5

Bathycentor kraesselini Saussure in Grandidier, 1892: pl. 21.

Bathycentor kraesselini - Shenefelt \& Marsh 1976: 1267. - Yu et al. 2016. - Belokobylskij 2018: 510. - Jasso-Martínez et al. 2019: 167.

\section{Material examined}

Lectotype

MADAGASCAR • + (designated by Belokobylskij 2018: 510); "Madagascar, Elliot", "Bathycentor kraesselini Madag.”, “o Bathycentor kraesselini Sauss., C. van Achterberg, 1978, Lectotype”; MNHN.

\section{Paralectotype}

MADAGASCAR • 1 o; "Madagas." [handwritten], "Museum Paris, Coll. J. De Gaulle 1919”, "O Ipodoryctes parallelus Granger, C. van Achterberg, 1978, Paralectotype", "conspecific with Lectotype Bathycentor kraesselini Sauss., Van Achterberg, 1978"; MNHN.

\section{Distribution}

Madagascar.

\section{Remarks}

The complete illustrated redescription of this species was recently published (Belokobylskij 2018: 510$512,767-771)$.

\section{Bathycentor zimbabwensis sp. nov. urn:1sid:zoobank.org:act:8F84EE58-82A7-4A59-AB7F-DD075A69D564}

Figs $6-7$

\section{Etymology}

Named after Zimbabwe, the country where the holotype was collected.

\section{Material examined}

\section{Holotype}

ZIMBABWE • ; “ “Zimbabwe, Harare, i.[19]82, Watsham [leg.]”; BMNH.

\section{Description}

\section{Female}

MeASuREments. Length of body $3.5 \mathrm{~mm}$; length of fore wing $3.0 \mathrm{~mm}$.

HEAD. Width 1.5 times its length along midline, 1.2 times maximum width of mesoscutum. Head behind eyes (dorsal view) distinctly evenly and roundly narrowed. Transverse diameter of eye (dorsal view) 1.7 times length of temple. Ocelli medium-sized, arranged in triangle with base 1.4 times its lateral sides. POL 2.0 times OD, 0.8 times OOL. Frons weakly but distinctly concave, with distinct and complete medial keel. Eye almost without emargination opposite antennal socket; height of eye 1.15 times width. Malar space 0.4 times height of eye, 0.8 times basal width of mandible. Width of face equal to height of eye and 1.4 times height of face with clypeus. Clypeus convex. Hypoclypeal depression round; its width 0.7 times distance from margin of depression to eye and 0.4 times width of face. Hypostomal flange narrow. Occipital carina not joined with hypostomal carina being obliterated at short distance upper base of mandible. Palps not long; maxillary palpus about as long as height of head (without mandibles). Head below eyes (front view) strongly roundly narrowed. 


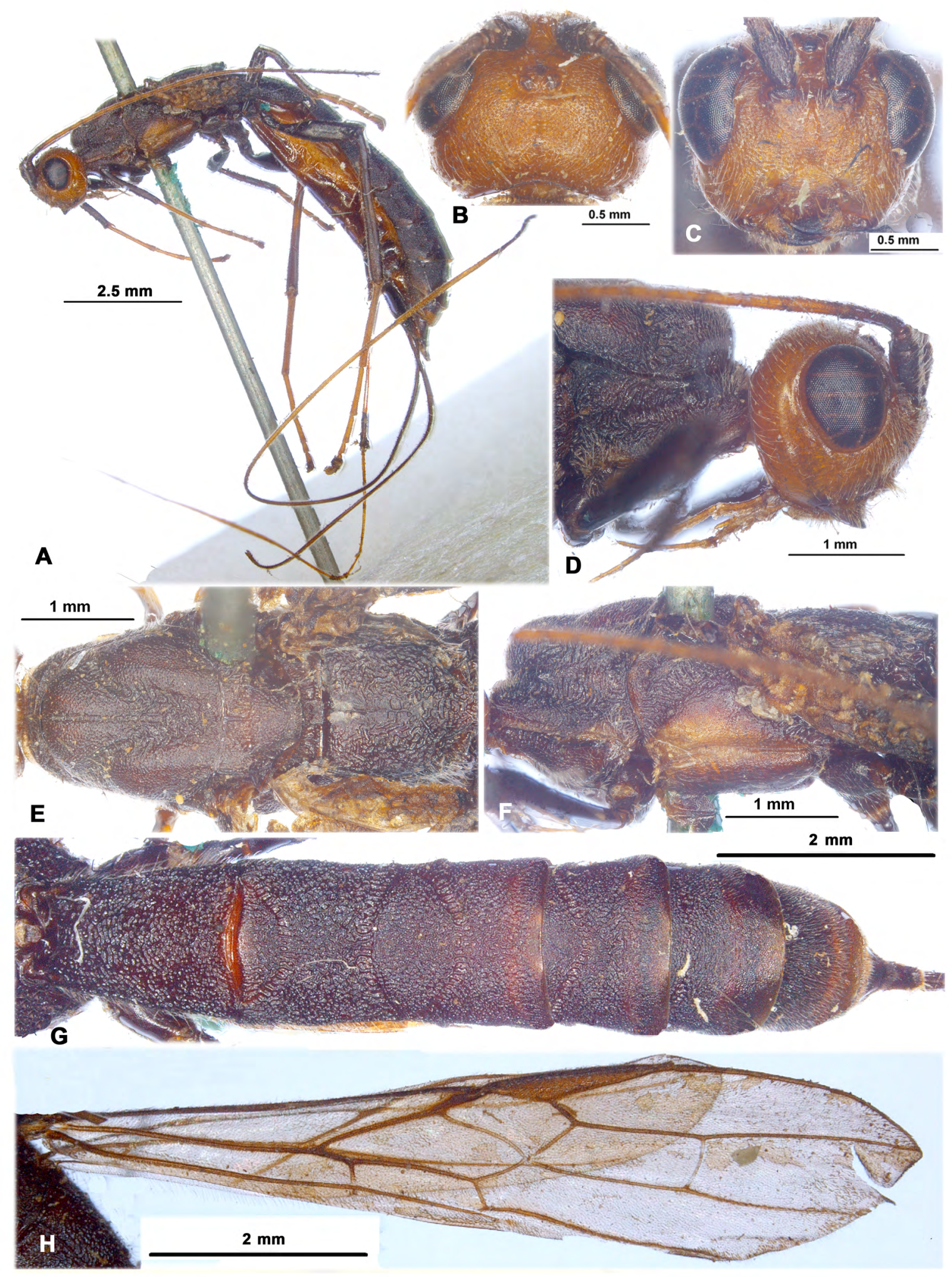

Fig. 5. Bathycentor kraesselini Saussure, 1892, $\uparrow$, lectotype, MNHN (A-G); Ipodoryctes parallellus Granger, 1949, + , paralectotype, MNHN (H). A. Habitus, lateral view. B. Head, dorsal view. C. Head, front view. D. Head, lateral view. E. Mesosoma, dorsal view. F. Mesosoma, lateral view. G. Metasoma, dorsal view. H. Wings. 


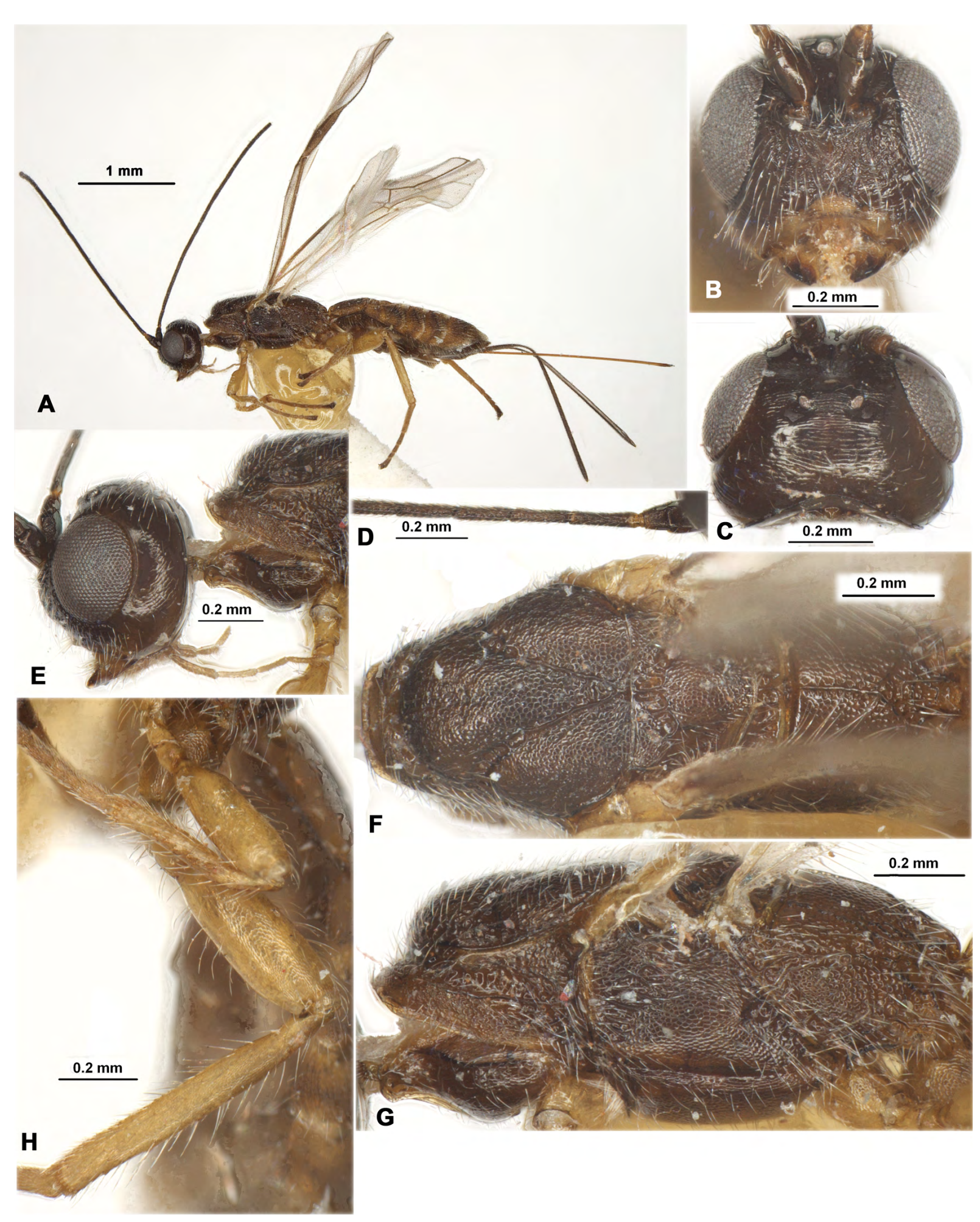

Fig. 6. Bathycentor zimbabwensis sp. nov.,, , holotype, BMNH. A. Habitus, lateral view. B. Head, front view. C. Head, dorsal view. D. Basal segments of antenna. E. Head, lateral view. F. Mesosoma, dorsal view. G. Mesosoma, lateral view. H. Hind leg. 

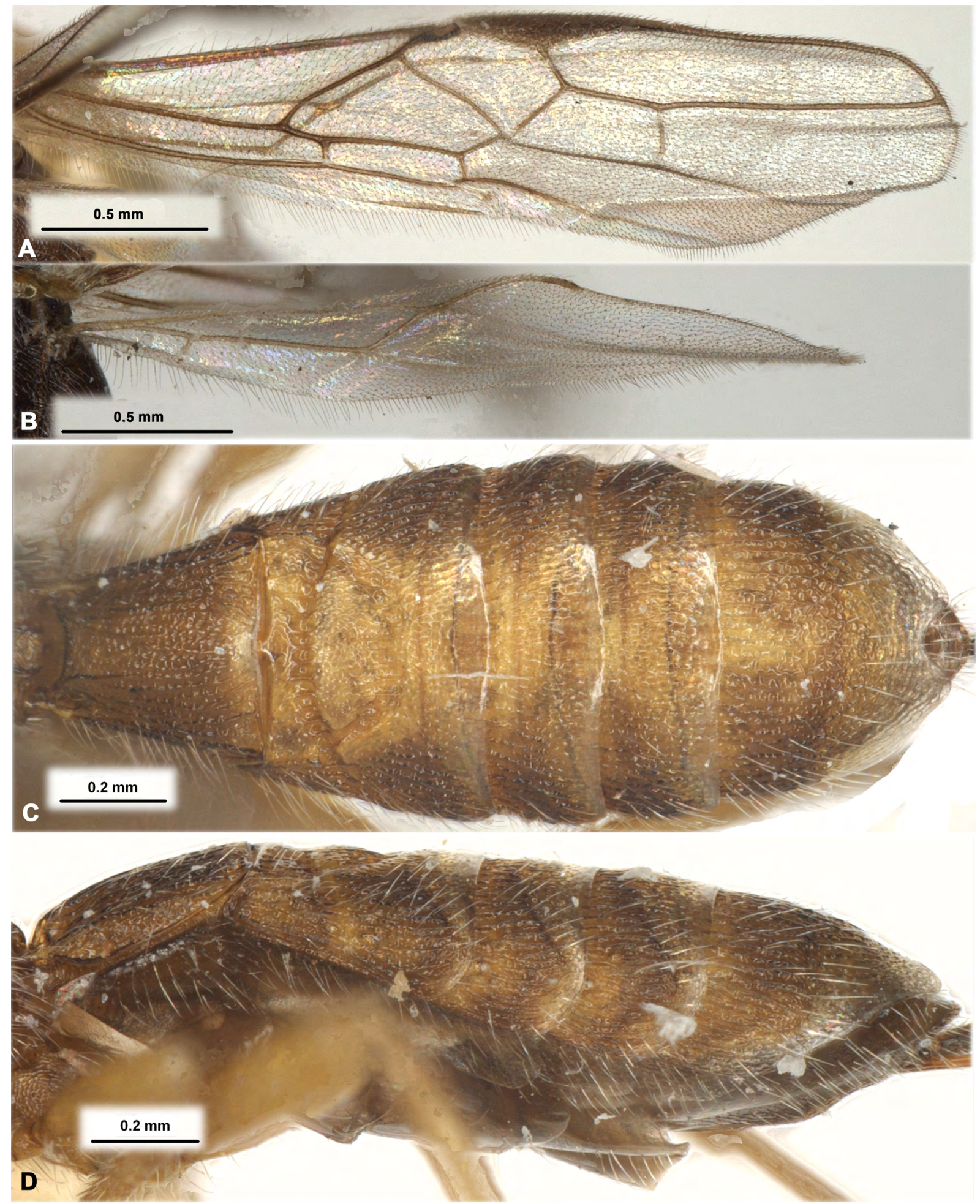

Fig. 7. Bathycentor zimbabwensis sp. nov.,, , holotype, BMNH. A. Fore wing. B. Hind wing. C. Metasoma, dorsal view. D. Metasoma, lateral view. 
Antennae slender, almost filiform, with more than 23 segments (apical segments missing). Length of scape 1.6 times its maximum width. First flagellar segment subcylindrical, weakly curved, its length 4.5 times apical width, 1.1 times length of second segment. Submedian segments of flagellum 3.4 times as long as their width.

Mesosoma. Length 2.1 times its height. Mesoscutum (dorsal view) almost as long as maximum width; its median lobe convex, rounded anteriorly, distinctly protruding forwards. Notauli deep anteriorly and shallower posteriorly, rather narrow and crenulate. Prescutellar depression shallow, long, with median and two lateral carinae, finely rugulose to smooth, 0.3 times as long as convex scutellum. Subalar depression rather narrow, crenulate-rugulose. Sternaulus rather deep anteriorly but shallow posteriorly, narrow, running along almost entire length of lower part of mesopleuron, weakly coriaceous. Metapleural lobe with sparse pubescence posteriorly.

Wings. Length of fore wing 4.1 times its maximum width. Pterostigma 5.3 times as long as wide. Metacarpus (1-R1) 1.2 times as long as pterostigma. Second radial abscissa (3-SR) 2.1 times as long as first abscissa (r), 0.35 times as long as straight third abscissa (SR1), 1.35 times as long as first radiomedial vein (2-SR). Second radiomedial (submarginal) cell short; its length 3.0 times maximum width, 1.1 times length of rather narrow brachial (subdiscal) cells. First medial abscissa (1-SR+M) weakly sinuate. Recurrent vein (m-cu) weakly antefurcal. Parallel vein (CU1a) of fore wing arising from posterior $1 / 3$ of vein (3-CU1) closed by brachial (subdiscal) cell posteriorly. Distance from nervulus (cu-a) to basal vein (1-M) 1.8 times length of nervulus (cu-a). Length of hind wing about 5.0 times its maximum width. First mediocubital abscissa $(\mathrm{M}+\mathrm{CU}) 0.7$ times as long as second and third abscissae (1-M) combined. Recurrent vein (m-cu) rather long, straight, oblique, interstitial, unsclerotized and discolorated.

LEGS. Hind femur with low and wide dorso-anterior protuberance, 2.9 times its maximum width. Hind tibia and tarsus weakly thickened. Hind tarsus as long as hind tibia. Hind basitarsus thickened, 0.7 times as long as second-fifth segments combined. Second segment of hind tarsus 0.5 times as long as basitarsus, 1.3 times as long as fifth segment (without pretarsus).

Metasoma. About as long as head and mesosoma combined. First tergite distinctly and linearly widened from base to subapex, then weakly narrowed towards apex. Maximum width of first tergite 1.8 times its minimum width; length of tergite 1.1 times its maximum width, 1.2 times length of propodeum. Length of second tergite (with posterior area) along midline 0.6 times its basal width, 1.8 times length of third tergite. Basal (anterior) area of second tergite short and wide, along midline 0.15 times, and posterior area 0.7 times as long as tergite length. Sixth tergite maximum length (with process) 1.9 times as long as fifth and 2.0 times as long as fourth tergite; posterior margin of sixth tergite with two short, wide and rounded apically median processes separated by deep emargination. Ovipositor sheath 1.2 times as long as metasoma, 1.5 times as long as mesosoma, 0.7 times as long as fore wing.

SCULPTURE AND PUBESCENCE. Vertex densely transversely striate, smooth narrow posteriorly and laterally; frons finely transversely striate with reticulation partly; face almost entirely rugulosereticulate; temple finely and curvedly obliquely striate, smooth anteriorly. Sides of pronotum densely rugose dorsally (with small smooth area) and ventrally, distinctly crenulate on middle oblique area. Mesoscutum entirely densely granulate-reticulate, with two distinct and strongly convergent posteriorly carinae along notauli in posterior half, rugose in small medio-posterior area. Scutellum entirely densely granulate. Mesopleuron entirely densely reticulate-areolate, becoming finer below and especially below sternaulus. Propodeum with large, wide and densely granulate-areolate basolateral areas; areola rather wide, coarsely rugose-striate, its length 1.1 times maximum width; basal carina of propodeum 0.45 times as long as propodeum, 1.6 times as long as anterior fork of areola. Hind coxa entirely densely granulate. Hind femur densely granulate-coriaceous, only coriaceous in lower $1 / 3$. First to fifth metasomal tergites entirely longitudinally striate, with dense additional reticulation between striae. Sixth tergite undulately longitudinally striate, with dense additional reticulation between striae in basal $3 / 4$, with 
distinct semicircular striae in posterior (apical) $1 / 4$. Vertex mainly with sparse short white setae, almost glabrous in narrow medioanterior part. Mesoscutum and mesopleurum entirely in not dense, short and semi-erect white setae. Hind tibia dorsally with long, rather dense semi-erect pale setae, its length $0.8-$ 1.0 times maximum submedian width of tibia.

CoLour. Head and mesosoma mainly dark reddish brown; metasoma yellowish brown, margins of fourth-sixth tergites subhyaline, but not white. Antennae black. Palpi brown basally and light brown apically. Legs yellowish brown, fifth tarsal segment brown, tibia basally same colour as other its part. Ovipositor sheath entirely dark brown. Fore wing entirely hyaline. Pterostigma entirely dark brown.

\section{Male \\ Unknown.}

\section{Diagnosis}

Bathycentor zimbabwensis sp. nov. is morphologically similar to B. trjapitzini Belokobylskij, 2018 from the Democratic Republic of the Congo, but it differs from the latter by having the vertex finely aciculate (vs coarsely striate), hind femur wide (vs narrow), second metasomal tergite short, 0.6 times as long as its basal width (vs longer, 0.9 times as long as its basal width), basal area of second tergite short and wide anteriorly (vs long and narrow), the posterior margin of the sixth metasomal tergite with deep median incisure and without pair of processes (vs without incisure but with pair of median processes), fore wing almost hyaline (vs infuscate), head and mesosoma dark brown (vs entirely light reddish brown), and body length short (vs long).

\section{Distribution}

Zimbabwe.

Genus Euryphrymnus Cameron, 1910 stat. nov.

Euryphrymnus Cameron, 1910: 100.

Rhaconotus (Euryphrymnus) - Belokobylskij 1996a: 154.

Euryphrymnus - Yu et al. 2016 (as synonym of Rhaconotus). - Jasso-Martínez et al. 2019: 167 (as synonym of Rhaconotus).

\section{Type species}

Euryphrymnus testaceiceps Cameron, 1910.

\section{Description (Figs 8-11)}

HEAD. Head not depressed, high, transverse. Ocelli arranged in weakly obtuse triangle. Frons almost flat, with very shallow median furrow. Eyes glabrous or rarely sparsely and shortly setose. Occipital carina dorsally complete, obliterate below and not joining ventrally with hypostomal carina at short distance. Malar suture absent. Clypeal suture distinct and complete. Hypoclypeal depression medium-sized, rounded. Postgenal bridge narrow, but distinct. Palpi relatively long; maxillary palps 6-segmented; labial palps 4-segmented, its third segment not shortened. Scapus wide and rather short, without apical lobe and basal constriction, its ventral margin (lateral view) not longer than dorsal margin. Pedicel weakly enlarged. First flagellar segment subcylindrical, almost straight, as long as second segment.

Mesosoma. Mesosoma not depressed and weakly elongate. Neck of prothorax rather short. Pronotum dorsally distinctly convex, with distinct pronotal carina situated submedially; propleural dorsoposterior flange long and rather wide. Mesonotum rather highly and roundly or gently roundly elevated above pronotum, mainly entirely granulate. Median lobe of mesonotum with or without median longitudinal furrow, without anterolateral corner. Notauli distinct and complete. Prescutellar depression relatively 
short, with several distinct carinae. Lateral longitudinal wing-like flanges on the level of prescutellar depression low but distinct. Scutellum convex, usually with fine lateral carinae. Metanotum with distinct, rather narrow and acuminate tooth (lateral view). Sternaulus (precoxal sulcus) more or less distinct, narrow, long, running almost along whole length of lower part of mesopleuron, often weakly sinuate. Prepectal carina distinct and complete, rather high below. Postpectal carina absent. Metapleural flange long, narrow, rounded apically, without dense setosity along its posterior margin. Propodeum with basolateral areas distinctly delineated by carinae, areola and other areas absent; lateral tubercles and propodeal bridge absent. Propodeal spiracles small and round.

WINGS. Pterostigma of fore wing wide and long. Radial vein (r) arising usually from middle of pterostigma. Radial (marginal) cell never shortened. Both radiomedial veins (2-SR, r-m) present. Second radiomedial (submarginal) cell medium-sized or rather long, rather wide, often widened towards apex. Recurrent vein (m-cu) postfurcal. Discoidal (discal) cell petiolate anteriorly, petiole (1-SR) short. Nervulus (cu-a) distinctly postfurcal. Mediocubital vein $(\mathrm{M}+\mathrm{CU} 1)$ distinctly sinuate. Brachial (subdiscal) cell gentlyroundly closed postero-apically weakly before recurrent vein $(\mathrm{m}-\mathrm{cu})$, without antero-posterior corner. Parallel vein (CU1a) always interstitial. Transverse anal veins (2A, a) absent. Hind wing with three hamuli. Radial (marginal) cell weakly widened in basal half and weakly narrowed in apical half, without transverse vein (r). Medial (basal) cell narrow, weakly widened towards apex. Nervellus (cu-a) present. Submedial (subbasal) cell short; first abscissa of mediocubital vein (M+CU) 0.4-0.5 times as long as second abscissa (1-M). Recurrent vein (m-cu) present, finely sclerotised or pigmented, weakly curved, distinctly oblique towards base of wing.

LEGS. Fore and middle tibiae with short and thick spines arranged in narrow short stripe. Middle tarsal segments rather short, but elongate. Hind coxa relatively long and wide, with distinct basoventral corner and tubercle. Hind femur relatively narrow, with low and wide dorsal protuberance. Hind basitarsus 0.6-0.7 times as long as second-fifth segments combined. Claws short and simple.

Metasoma. With only five visible tergites, with separated laterotergites on second-fifth tergites. Acrosternite of first segment short, about 0.2 times as long as first tergite, its apical margin situated before level of spiracles. First tergite not petiolate, rather long and distinctly widened towards apex; with distinct dorsope; with relatively long or only distinct basolateral process; spiracles situated in basal 0.3 of tergite. Second tergite movably connected with first tergite, with short or rather long basal area, delineated posteriorly by distinct and usually complete semi-circular or sinuate posterior sulcus; apical area always present, long, lenticular, distinctly separated by deep and curved furrow anteriorly and second suture posteriorly. Second suture deep, rather wide, complete, evenly curved. Fourth and fifth tergites with deep and crenulate subbasal transverse furrows. Fifth tergite distinctly enlarged, longer than previous tergite, covered following apical segments. Ovipositor sheath shorter than metasoma.

\section{Diagnosis}

Members of this genus with five metasomal tergites are very similar to the nominative genus of the tribe, Rhaconotus Ruthe, 1854 but can be distinguished from the latter by the presence of a transverse or semi-oval basal area on the second tergite distinctly delineated by a deep furrow, and always together with an apical lenticular area sharply delineated anteriorly by deep sulcus and posteriorly by a second suture. A similar structure on the second metasomal tergite is also know in species of Bathycentor and Ipodoryctes, though members of these genera always have more than five dorsally visible metasomal tergites.

\section{Composition}

Euryphrymnus basoareolus (Belokobylskij, 2001) comb. nov. (OR); E. gaullei (Granger, 1949) comb. nov. (AF); E. testaceiceps Cameron, 1910 (OR). 


\section{Hosts}

Unknown.

\section{Distribution}

Afrotropical and Oriental regions.

\section{Key to species of Euryphrymnus}

1. Fifth tergite on posterior margin with two wide and distinct submedial processes and emargination between it. Body length $6.0 \mathrm{~mm}$. Madagascar E. gaullei (Granger, 1949) comb. nov.

- Fifth tergite on posterior margin without submedial processes and emargination between it, evenly rounded (Figs 9C; 11B)

2. Mesoscutum densely granulate and with distinct rugosity around notauli, setose on wide area along notauli and along sides of mesoscutum (Fig. 8F). Mesopleuron entirely and densely setose (Fig. 8G). Apical area of second metasomal tergite entirely coarsely striate (Fig. 9C). Fifth tergite entirely striate-reticulate, 1.6 times as long as fourth tergite (Fig. 9C). Body length $5.1 \mathrm{~mm}$. Indonesia

E. testaceiceps Cameron, 1910

- Mesoscutum densely granulate and without rugosity around notauli, mainly glabrous, with only few setae medio-posteriorly (Fig. 10G). Mesopleuron mainly glabrous (Fig. 10F). Apical area of second metasomal tergite almost entirely smooth (Fig. 11B). Fifth tergite striate in basal half and smooth in posterior half, 1.2-1.3 times as long as fourth tergite (Fig. 11B). Body length 2.5-2.7 mm, Malaysia, Vietnam E. basoareolus (Belokobylskij, 2001) comb. nov.

\section{Euryphrymnus testaceiceps Cameron, 1910}

Figs 8-9

Euryphrymnus testaceiceps Cameron, 1910: 100.

Euryphrymnus testaceiceps - Viereck 1914: 57.

Rhaconotus testaceiceps - Shenefelt \& Marsh 1976: 1342. - Belokobylskij 2001: 133. - Yu et al. 2016. - Jasso-Martínez et al. 2019: 167.

\section{Material examined}

\section{Holotype}

INDONESIA • '; "Siluas Sambas, W. Borneo, 22/26.7.07, Micholitz", "Euryphrymnus testaceiceps Cam., Type. Borneo"; MHBG.

\section{Description}

Female

Measurements. Body length $5.1 \mathrm{~mm}$; fore wing length $3.6 \mathrm{~mm}$.

HEAD. Head width 1.5 times its median length, 1.35 times width of mesoscutum. Head behind eyes (dorsal view) distinctly and roundly narrowed. Transverse diameter of eye almost 2 times length of temple. Ocelli small, arranged in almost equilateral triangle; POL almost equal to OD, 0.35 times OOL. Eye with very short and sparse setae, very weakly emarginated opposite antennal sockets, 1.15 times as high as broad. Malar space 0.5 times height of eye, 0.9 times basal width of mandible. Face width 1.2 times height of eye and 1.45 times height of face and clypeus combined. Malar suture absent. Upper margin of clypeus situated almost on lower level of eyes. Hypoclypeal depression small and round, its width 0.7 times distance from edge of depression to eye, 0.35 times width of face. Clypeus with distinct, but short 


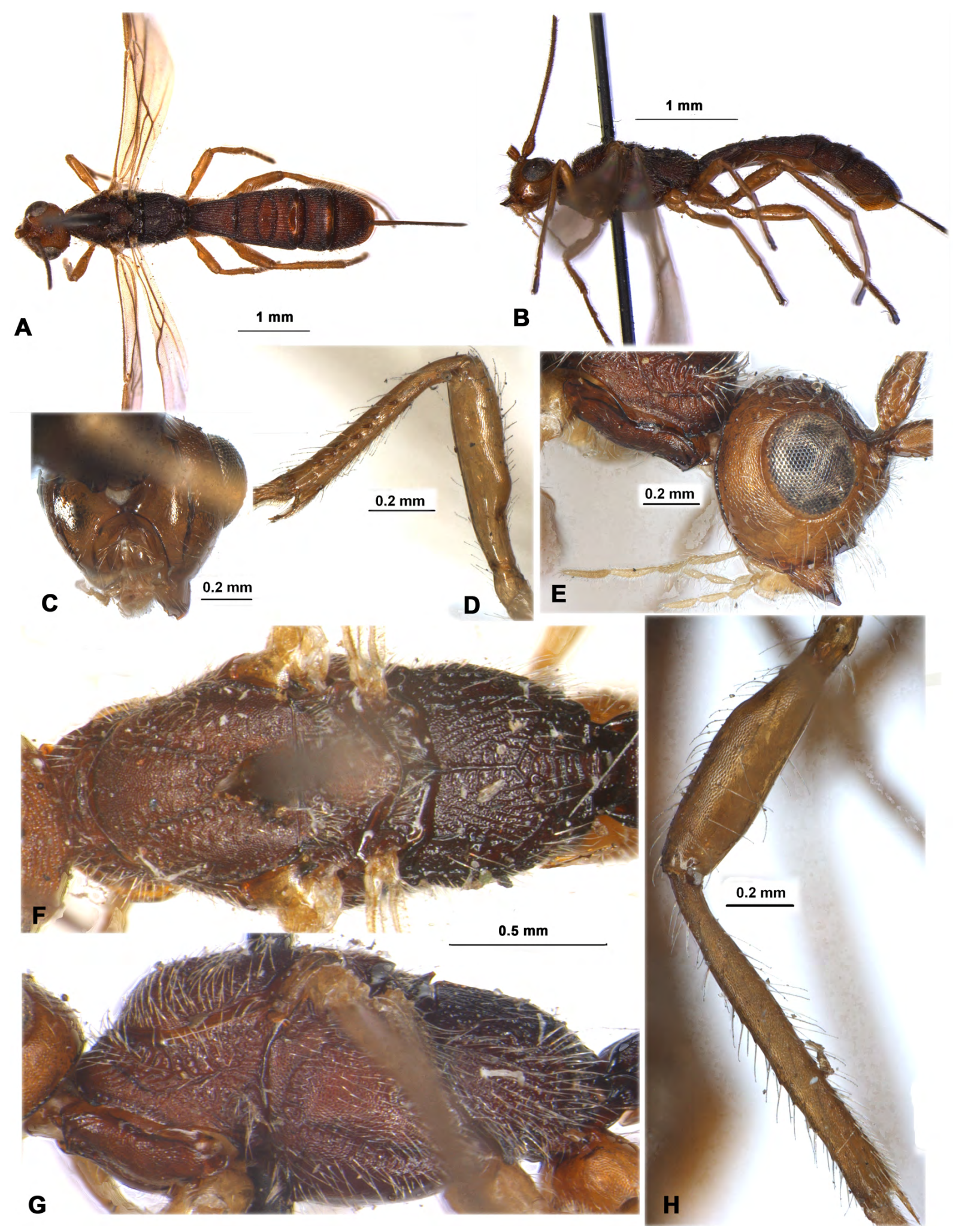

Fig. 8. Euryphrymnus testaceiceps Cameron, 1910, + , holotype, MHBG. A. Habitus, dorsal view. B. Habitus, lateral view. C. Head, latero-posterior view. D. Fore leg. E. Head, lateral view. F. Mesosoma, dorsal view. G. Mesosoma, lateral view. H. Hind leg. 

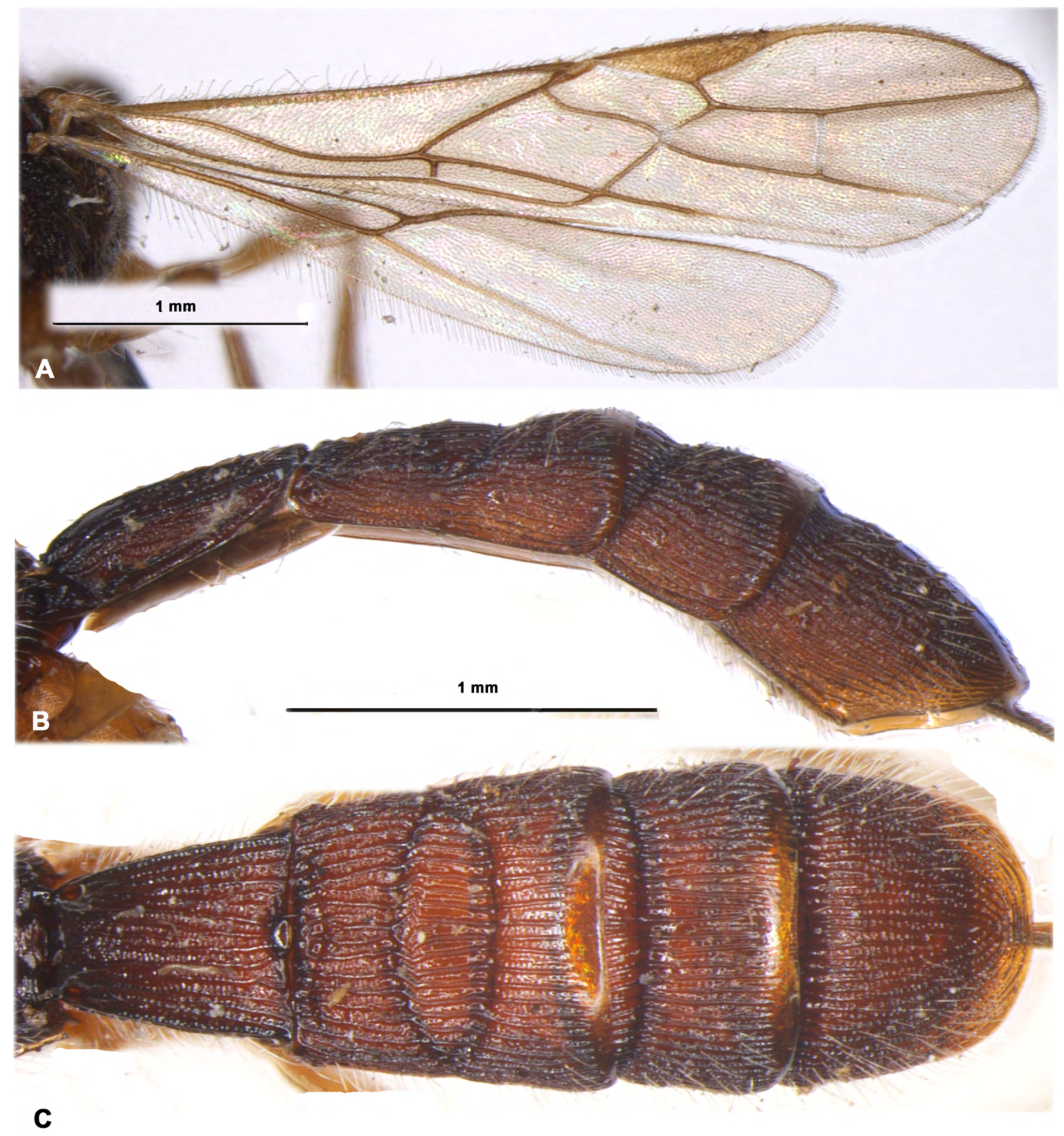

Fig. 9. Euryphrymnus testaceiceps Cameron, 1910, + , holotype, MHBG. A. Wings. B. Metasoma, lateral view. C. Metasoma, dorsal view. 
ventral flange. Occipital carina not fused ventrally with hypostomal carina being obliterated shortly upper base of mandible. Vertex distinctly convex. Head below eyes strongly and almost linearly narrowed. Antennae rather slender, filiform, more than 9-segmented (most part of apical segments missing). Length of scapus almost twice its maximum width. First flagellar segment 4.8 times as long as its apical width, 1.1 times as long as second segment.

Mesosoma. Length 2.0 times its height. Pronotal carina rather distinct, distances from carina to both sides of pronotum subequal. Mesoscutum rather highly and roundly elevated above pronotum, 1.1 times as long as wide. Notauli entirely deep, complete, rather wide, distinctly crenulate. Median lobe of mesoscutum with shallow anteriorly and deep posteriorly median furrow. Prescutellar depression rather deep, short, distinctly and roundly curved posterolaterally, with five distinct carinae, finely rugulose, 0.25 times as long as convex scutellum. Sternaulus (precoxal furrow) rather shallow, narrow, weakly sinuate, distinctly and densely crenulate, running along entire lower part of mesopleuron. Prepectal carina without widened lobes opposite fore coxae. Subalar depression rather shallow, narrow, distinctly crenulate and almost smooth between crenulae. Metanotum with rather long, narrow and pointed tooth directed backward. Metapleural lobe long, narrow, rounded apically, without dense pubescence. Mesopleural suture entirely and distinctly crenulate.

WINGS. Fore wing 4.2 times its maximum width. Radial (marginal) cell not shortened. Metacarpus (1R1) 1.2 times as long as pterostigma. Radial vein (r) arising from middle of pterostigma. First radial abscissa (r) forming very obtuse angle with second abscissa (3-SR). Second radial abscissa (3-SR) 4.0 times as long as first abscissa (r), 0.55 times as long as weakly curved third abscissa (SR1), 1.85 times as long as first radiomedial vein (2-SR). Second radiomedial (submarginal) cell rather long, widened distally, 2.5 times as long as wide, as long as brachial (subdiscal) cell. First medial abscissa (1-SR+M) distinctly sinuate. Recurrent vein (m-cu) weakly postfurcal. Discoidal (discal) cell 2.9 times as long as maximum width. Mediocubital vein (M+CU1) rather distinctly curved to anal vein (1-1A) in distal half. Nervulus (cu-a) postfurcal, distance between nervulus (cu-a) and basal (1-M) vein 0.5 times nervulus (cua) length. Brachial (subdiscal) cell rather wide, gently-roundly closed weakly before level of recurrent vein (m-cu); posterior bulla and posterior abscissa of anal vein (2-1A) (behind brachial vein (CU1b)) absent. Hind wing 5.7 times as long as wide. First costal abscissa 0.55 times as long as second abscissa. First abscissa of mediocubital vein $(\mathrm{M}+\mathrm{CU}) 0.4$ times as long as second abscissa (1-M). Recurrent vein $(\mathrm{m}-\mathrm{cu})$ very fine, unsclerotised, curved, postfurcal.

LEGS. Hind coxa with distinct basoventral tooth, 1.5 times as long as wide (with basoventral tubercle). Hind femur with not high dorsal protuberance, length of femur 3.3 times maximum width. Hind tarsus almost as long as hind tibia. Hind basitarsus 0.7 times combined length of second-fifth segments. Second tarsal segment 0.45 times as long as basitarsus, 2.0 times as long as fourth segment, 1.25 times as long as fifth segment (without pretarsus).

Metasoma. Length 1.2 times as long as head and mesosoma combined, with five visible tergites (following tergites not protruding behind fifth one). First tergite without subbasal lateral processes. Maximum width of first tergite about 2 times its basal width; its length 1.35 times apical width, 1.3 times length of propodeum. Second tergite with short basal area, delineated posteriorly by curved furrow; with deep, narrow and curved transverse subposterior furrow very distinctly separated wide apical area median length of area 0.9 times length of rest part of tergite. Median length of second tergite 0.75 times its basal width, 1.5 times length of third tergite. Second suture deep, rather wide, distinctly regularly curved. Fifth tergite enlarged, distinctly convex in posterior margin, without median emargination and postero-ventral lobes. Fifth tergite 1.6 times as long as fourth tergite, 1.75 times as long as third tergite. Ovipositor sheath 0.55 times as long as metasoma, 1.3 times as long as hind tibia, 0.9 times as long as mesosoma, 0.4 times as long as fore wing. 
SCUlPTURE AND PUBESCENCE. Vertex densely distinctly granulate, with distinct and weakly undulate transverse striae; frons densely granulate and with distinct striae directed from lateral sides towards middle; face densely and rather finely granulate, almost smooth on narrow median area; temple almost entirely granulate. Mesoscutum entirely densely and distinctly granulate, with distinct and rather long rugosity along notauli and laterally, with short rugosity in medioposterior 0.3 of mesoscutum. Scutellum densely and distinctly granulate. Mesopleuron densely and entirely granulate, with fine rugulosity laterally. Metapleuron densely rugose-reticulate with granulation. Propodeum with distinctly delineated, densely striate with granulation large basolateral areas, with rather distinct, wide and coarsely rugosestriate areola. Hind coxa densely and distinctly granulate in upper half, finely granulate in lower half. Hind femur reticulate-coriaceous in upper half, finely coriaceous or almost smooth in lower half. First tergite with distinct, complete and weakly convergent dorsal carinae, distinctly coarsely striate with dense fine reticulation between striae, with additional dense granulation in anterior 0.3 of tergite. Second tergite entirely coarsely and undulately striate with dense fine rugulosity between striae. Third and fourth tergites in basal 0.8 distinctly densely linearly striate and with additional reticulation, narrowly smooth apically. Fifth tergite entirely, densely, coarsely and curvedly striate, with densely and distinct reticulate between striae. Second-fourth tergites laterally entirely densely striate with granulation. Vertex entirely with rather short, more or less dense and semi-erect pale setae. Mesoscutum dense, rather short and semierect setae arranged widely along notauli and laterally, with wide glabrous median areas. Mesopleuron entirely covered by dense pale setae. Hind tibia dorsally with rather short, more or less dense, semi-erect pale setae; length of these setae 0.6-0.8 times maximum width of hind tibia.

CoLour. Head light reddish brown. Mesosoma reddish brown in anterior half and dark reddish brown in posterior half. Metasoma reddish brown. Antenna reddish brown to dark reddish brown, two basal segments light reddish brown. Palps brownish yellow. Legs brownish yellow, hind coxa light reddish brown, apical segments of all tarsi dark brown. Ovipositor sheath mainly black, reddish brown in its basal 0.3 . Fore wing evenly faintly infuscate. Pterostigma mainly yellowish brown, yellow in its basal 0.4 .

\section{Male \\ Unknown.}

\section{Distribution}

Indonesia (Borneo).

Euryphrymnus basoareolus (Belokobylskij, 2001) comb. nov.

Figs 10-11

Rhaconotus basoareolus Belokobylskij, 2001: 102.

Rhaconotus basoareolus - Yu et al. 2016.

\section{Material examined}

Holotype

VIETNAM - ; ; Province Gia Lai - Con Tum, Buon Luoi, 20 km N of Kannack; 17-20 Nov. 1988;

A. Sharkov leg.; ZISP.

\section{Paratypes}

VIETNAM • 1 क; same collection data as for holotype; 8-16 Nov. 1988 • 1 क; Province Ha Son Binh, Ky Son, Cao Phong; 25 Oct. 1990, S. Belokobylskij leg.; forest; ZISP. 


\section{BELOKOBYLSKIJ S.A. \& ZALDÍVAR-RIVERÓN A., Reclassification of the Rhaconotini}

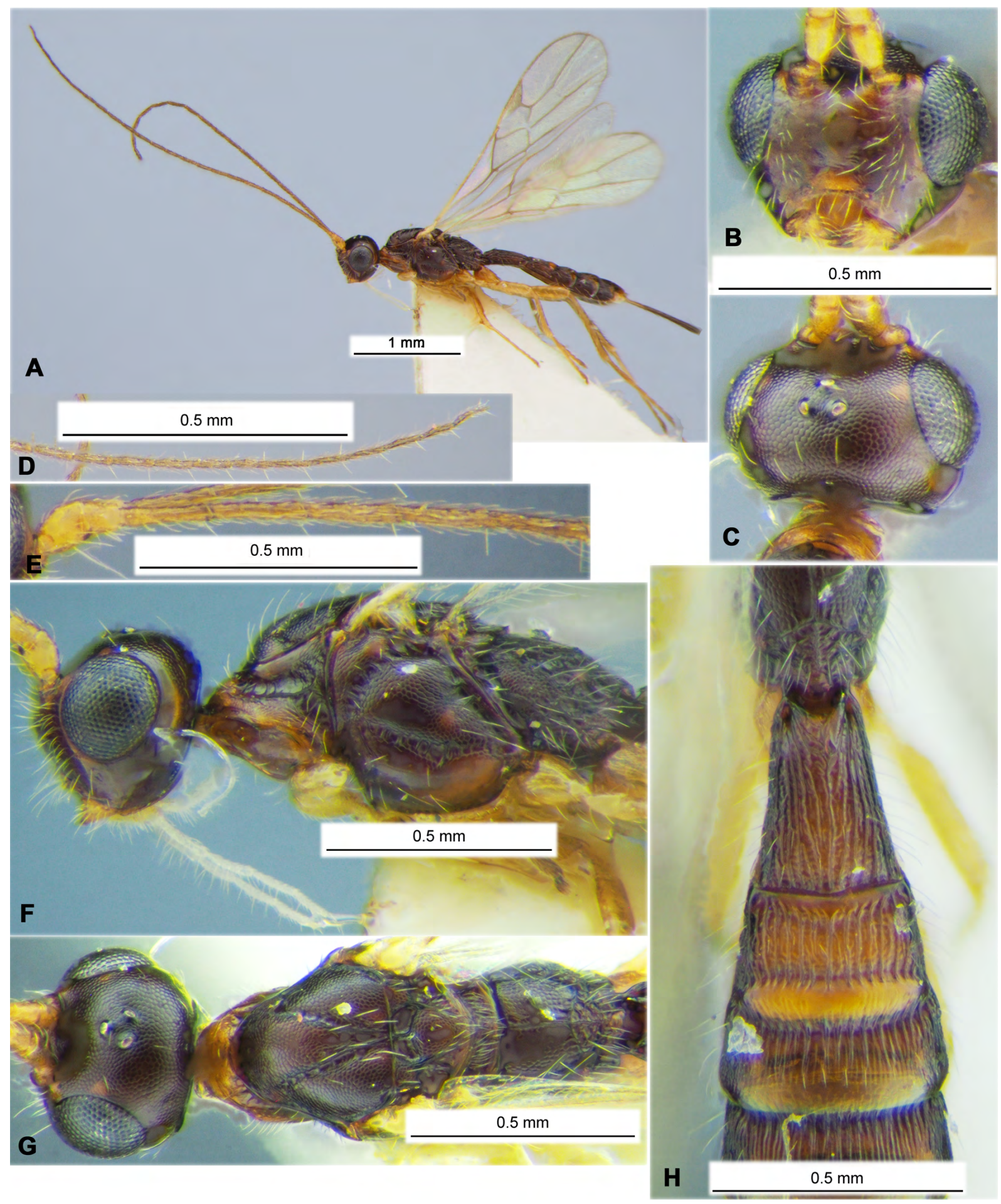

Fig. 10. Euryphrymnus basoareolus (Belokobylskij, 2001) comb. nov., $q$, holotype, ZISP. A. Habitus, lateral view. B. Head, front view. C. Head, dorsal view. D. Apical segments of antenna. E. Basal segments of antenna. F. Mesosoma, lateral view. G. Mesosoma, dorsal view. H. Propodeum and first to third metasomal tergites, dorsal view. 

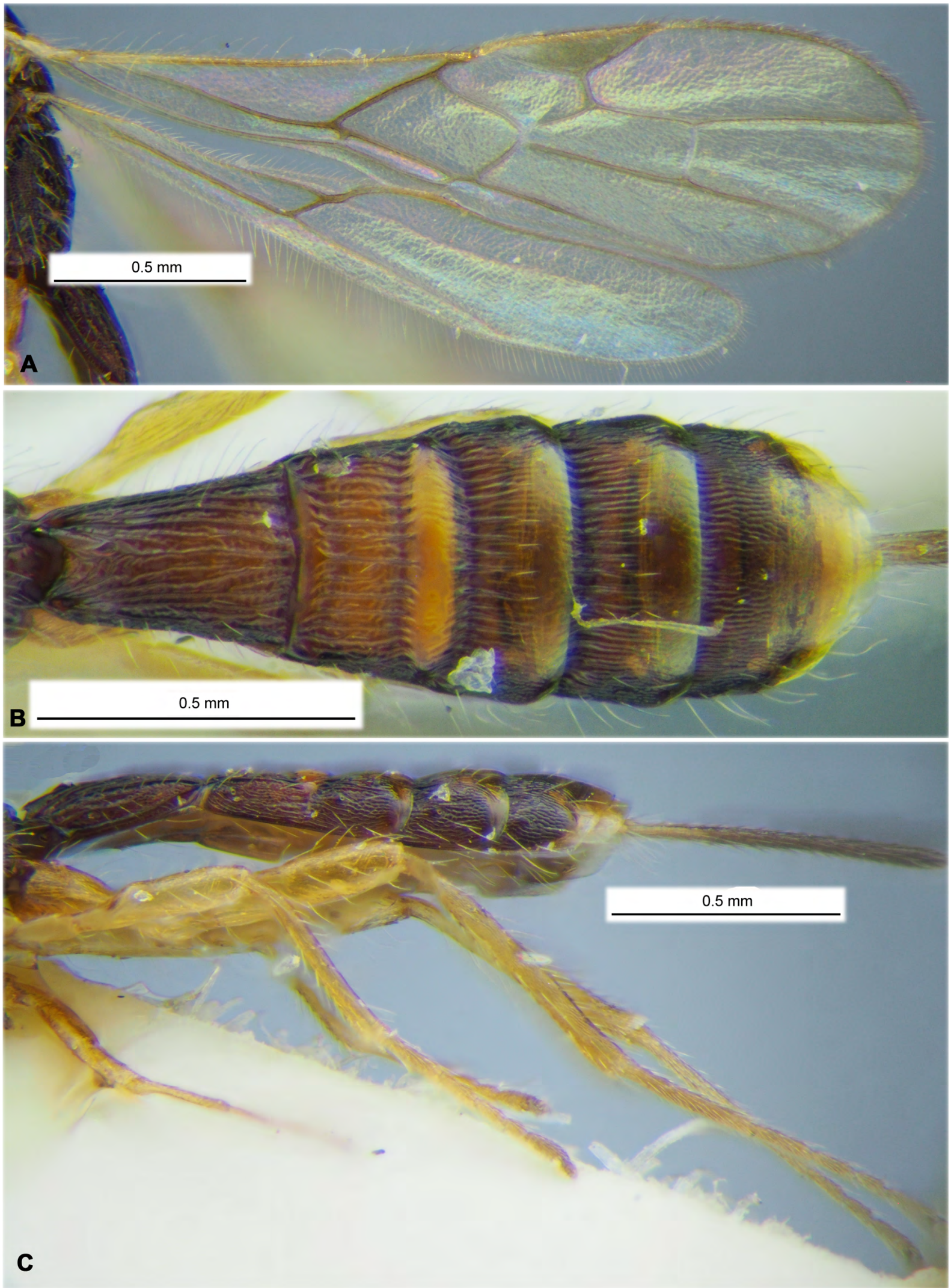

Fig. 11. Euryphrymnus basoareolus (Belokobylskij, 2001) comb. nov.,, , holotype, ZISP. A. Wings. B. Metasoma, dorsal view. C. Metasoma and ovipositor, lateral view. 


\section{Other material}

MALAYSIA • 1 क; Sarawak, 3 div. Hillpadi, NB-2-2; 22 Sep. 1974; D. Munroe leg.; CNCI • 1 \%; Sarawak, Santubang; 3-4 Jan. 1978; B. Bendell leg.; CNCI.

\section{Description}

Female

Measurements. Body length 2.5-2.7 mm; fore wing length 2.0-2.3 mm.

HEAD. Head width 1.4-1.5 times its median length, 1.1-1.2 times width of mesoscutum. Head behind eyes (dorsal view) roundly narrowed. Transverse diameter of eye 2.0-2.5 times length of temple. Ocelli small, arranged in almost equilateral triangle; POL 1.2-1.3 times OD, 0.35 times OOL. Eye glabrous, 1.1-1.2 times as high as broad. Malar space 0.40-0.45 times height of eye, almost equal to basal width of mandible. Face width 1.1-1.2 times height of eye and 1.3-1.4 times height of face and clypeus combined. Malar suture present, complete but weak. Upper margin of clypeus situated slightly upper than lower border of eyes or on same level. Hypoclypeal depression subround, its width $0.7-0.8$ times distance from edge of depression to eye, $0.3-0.4$ times width of face. Occipital carina not fused ventrally with hypostomal carina being shortly obliterated upper base of mandible. Vertex distinctly convex. Head below eyes weakly and weakly-roundly narrowed (front view). Antennae rather slender, filiform, 25-26-segmented, 1.3-1.4 times as long as body. Length of scapus 1.5-1.7 times its maximum width. First flagellar segment 4.7-5.0 times as long as its apical width, $0.80-0.95$ times as long as second segment. Penultimate segment 4.3-4.5 times as long as wide, $0.8-0.9$ times as long as first segment, 0.8 times as long as apical segment.

Mesosoma. Length 1.8-2.0 times its height. Pronotal carina distinct, distances from carina to anterior and posterior margins of pronotum subequal. Notauli entirely deep, complete, sparsely crenulate. Median lobe of mesoscutum without median furrow. Prescutellar depression rather deep, weakly and roundly widened postero-laterally, with distinct median carina, smooth, 0.3 times as long as weakly convex scutellum. Sternaulus (precoxal furrow) rather shallow, crenulate, weakly sinuate, running along entire lower part of mesopleuron. Prepectal carina distinct, rather wide ventrally but without widened lobes opposite fore coxae. Subalar depression deep, narrow, crenulate. Metanotum with short and acuminate posteriorly tooth (lateral view).

WINGS. Fore wing length 3.5-3.6 times its maximum width. Radial (marginal) cell not shortened. Metacarpus (1-R1) 1.3-1.4 times as long as pterostigma. First radial abscissa (r) forming distinct obtuse angle with second abscissa (3-SR). Second radial abscissa (3-SR) 3.3-3.7 times as long as first abscissa (r), 0.5-0.6 times as long as the straight third abscissa (SR1), 1.5-2.2 times as long as first radiomedial vein (2-SR). Second radiomedial (submarginal) cell weakly widened distally, its length 3.0-3.5 times maximum width, 1.4-1.6 times length of narrow brachial (subdiscal) cell. First medial abscissa (1$\mathrm{SR}+\mathrm{M})$ weakly sinuate. Mediocubital vein $(\mathrm{M}+\mathrm{CU} 1)$ weakly curved to anal vein (2-1A) in distal half. Nervulus (cu-a) postfurcal, distance between nervulus (cu-a) and basal (1-M) vein almost equal to nervulus (cu-a) length. Brachial (subdiscal) cell strongly and almost linearly closed distinctly before level of recurrent vein; posterior abscissa of anal vein (2-1A) (behind brachial vein (CU1b)) indistinct. Hind wing 5.8-6.2 times as long as wide. First costal abscissa $0.4-0.5$ times as long as second abscissa. First abscissa of mediocubital vein $(\mathrm{M}+\mathrm{CU})$ about 0.4 times as long as second abscissa (1-M). Recurrent vein $(\mathrm{m}-\mathrm{cu})$ fine and strongly desclerotised.

LEGS. Hind coxa with rather distinct basoventral tubercle, 1.4-1.6 times as long as its wide (with tubercle). Hind femur with dorsal protuberance, its length 3.5-3.8 times maximum width. Hind tarsus $1.10-1.15$ times as long as hind tibia. Hind basitarsus about 0.7 times combined length of second-fifth 
segments. Second tarsal segment $0.40-0.45$ times as long as basitarsus, $1.2-1.3$ times as long as fifth segment (without pretarsus).

Metasoma. Length slightly longer than head and mesosoma combined. First tergite distinctly, evenly and linearly widened towards subapex. Maximum width of first tergite almost 2 times its basal width, its length 1.3-1.4 times apical width, about 1.4 times length of propodeum. Median length of second tergite $0.8-0.9$ times its basal width, 1.8-2.0 times length of third tergite; basal area medially 0.2 times as long as remaining part of second tergite (with apical area); apical area medially 0.6 times as long as remaining part of tergite (with basal area). Fifth tergite moderately large, with weakly and regularly rounded posterior margin, without median emargination and postero-ventral lobes. Fifth tergite 1.2-1.3 times as long as fourth tergite, $1.5-1.6$ times as long as third tergite. Ovipositor sheath $0.4-0.6$ times as long as metasoma, $0.6-0.8$ times as long as mesosoma, about 0.3 times as long as fore wing.

SCULPTURE AND PUBESCENCE. Vertex and frons finely and densely granulate; face very finely granulate, smooth medially; temple smooth or very finely coriaceous along occipital carina. Mesonotum entirely densely and finely coriaceous, with two convergent medio-posteriorly striae and without rugosity between them. Scutellum finely granulate. Mesopleuron coriaceous, granulate posteriorly. Metapleuron densely granulate, rugose posteriorly. Propodeum with median carina along its entire length; basolateral areas distinctly delineated, densely granulate or coriaceous; remainder of propodeum rugulose, with distinct, at least medially, transverse median carina, areola more or less distinctly delineated. Hind coxa granulate in upper half, smooth in lower half. Hind femur mainly coriaceous, almost smooth below. First metasomal tergite with dorsal carinae fused in basal 0.2 of tergite, then united carina following to apex of tergite. First and second tergites striate, second tergite smooth basally, finely or very finely rugulose in apical area. Third and fourth tergites in its basal 0.7 striate with rugulosity between striae, smooth in apical $1 / 3$. Fifth tergite striate in basal its $0.2-0.3$, rugulose-coriaceous medially, almost smooth in apical $1 / 3$, but sometimes tergite here finely or very finely transversely striate-coriaceous. Second-fourth tergites laterally coriaceous-striate (especially below) or only coriaceous with granulation partly. Vertex with rather long and very sparse semi-erect setae directed forward. Mesoscutum with rather dense, semierect, white setae arranged along notauli and marginally. Hind tibia dorsally with semi-erect and rather sparse white setae; length of these setae 1.2-1.5 times maximum width of hind tibia.

CoLour. Body dark reddish brown and partly reddish brown; pronotum and metasoma dorsally partly light reddish brown, sometimes metasoma entirely medially light reddish brown. Antenna brown to almost black, two basal segments brownish yellow. Palps pale yellow. Legs brownish yellow or yellow; hind coxae sometimes darker basally or entirely. Ovipositor sheath dark brown, yellow or pale brown basally. Wings almost hyaline. Pterostigma brown or pale brown, yellow in basal quarter.

\section{Male}

Unknown.

\section{Distribution}

Vietnam, Malaysia.

Genus Grangerdoryctes Belokobylskij, 2005

Grangerdoryctes Belokobylskij, 2005: 206.

Grangerdoryctes - Yu et al. 2016. — Jasso-Martínez et al. 2019: 165.

\section{Type species}

Aivalykus niger Granger, 1949, by original designation. 


\section{Description (Figs 12-13)}

HEAD. Head not depressed, rather high, weakly transverse. Ocelli arranged in distinctly obtuse triangle. Frons flat, without median keel or furrow. Eyes glabrous. Occipital carina dorsally complete, not joined ventrally with hypostomal carina at rather long distance. Malar suture absent. Clypeal suture present, complete, rather deep entirely. Clypeus with short ventral flange. Hypoclypeal depression medium size, oval. Palps medium length; maxillary palps 6-segmented, its sixth (apical) segment about as long as fifth segment; labial palps short. Scapus very wide and rather long, with short apical fringe, without basal constriction, its ventral margin (lateral view) not longer than dorsal margin. First flagellar segment subcylindrical, weakly curved, shorter than second segment. Apical segment weakly pointed apically and without spine.

Mesosoma. Mesosoma weakly depressed and long. Neck of prothorax long. Pronotum dorsally distinctly convex, with distinct, high and pointed apically, not fused medioposteriorly pronotal carina. Pronope absent. Propleural dorsoposterior lobe long and wide. Mesonotum entirely densely granulate-reticulate, not highly and gently-roundly elevated above pronotum. Median lobe of mesonotum without median longitudinal furrow, without anterolateral corners. Notauli distinct and complete. Tegula short, widened distally, weakly convex along its posterior margin. Prescutellar depression long, with distinct median carina. Lateral longitudinal wing-like flanges on the level of prescutellar depression short and rather low. Scuto-scutellar suture distinct and complete. Scutellum convex, without distinct lateral carinae. Mesopleural pit deep, wide, subround, connected directly with mesopleural suture. Metanotum with very short, wide and subpointed median tooth. Sternaulus (precoxal sulcus) rather deep, narrow, long, running along all length of lower part of mesopleuron, weakly curved. Prepectal carina distinct and complete, rather high below, laterally following to sternaulus. Postpectal carina absent. Metapleural flange rather short, wide, subrounded apically. Propodeum without delineated areas; lateral tubercles absent; propodeal bridge absent. Propodeal spiracles small and subround.

WINGS. Pterostigma of fore wing narrow and long. Radial vein (r) arising from middle of pterostigma. Radial (marginal) cell not shortened. Second radiomedial vein (r-m) absent. Radial (marginal) cell long and narrow. Recurrent vein (m-cu) strongly postfurcal. Discoidal (discal) cell wide and short, petiolate anteriorly, petiole (1-SR) short. Nervulus (cu-a) distinctly postfurcal. Brachial (subdiscal) cell closed postero-apically distinctly before recurrent vein $(\mathrm{m}-\mathrm{cu})$, without antero-posterior corner. Parallel vein (CU1a) interstitial. Transverse anal veins (2A, a) absent. Hind wing with three hamuli. First abscissa of costal vein $(\mathrm{C}+\mathrm{SC}+\mathrm{R}) 0.45$ times as long as second abscissa $(1-\mathrm{SC}+\mathrm{R})$. Radial vein $(\mathrm{SR})$ strongly unclerotised, arising from costal vein $(2-\mathrm{SC}+\mathrm{R})$ and rather far separating from basal vein $(1 \mathrm{r}-\mathrm{m})$. Radial (marginal) cell unclear delineated. Medial (basal) cell very weakly widened towards apex from base, long and narrow, about 19.0 times as long as width, 0.4 times as long as hind wing. Nervellus (cu-a) present. Submedial (subbasal) cell short. First abscissa of mediocubital vein $(\mathrm{M}+\mathrm{CU}) 0.35$ times as long as second abscissa (1-M). Recurrent vein (m-cu) absent.

LeGs. Fore and middle tibiae with a few short and thick spines arranged in one row. Fore tarsus 1.5 times as long as for tibia. Middle tarsal segments long. Hind coxa rather long and wide, with distinct basoventral corner and tubercle. All femora with more or less distinct, wide and low dorsal protuberances. Hind femur wide. Hind basitarsus about 0.5 times as long as second-fifth segments combined. Claws thick, short and simple.

Metasoma. Widened to base of fourth segment, then weakly narrowed. First tergite not petiolate, rather long and wide. Acrosternite of first tergite short, about 0.2 times as long as first tergite, its apical margin situated before level of spiracles. Dorsope of first tergite shallow and small; basolateral processes absent; spiracular tubercles very small, spiracles situated in basal 0.3 of tergite; dorsal carinae indistinct. First and second tergite not fused immovably. Second tergite without basal area. Second suture absent; 
third tergites with wide, semicircular and coarsely crenulate concave furrow. Similar distinctly concave, widely situated, crenulate furrow developed on fourth and fifth tergites. Present six visible dorsally tergites with separated laterotergites (epipleura); other segments weakly protruding behind sixth one. Six tergites rather coarsely sclerotised, not enlarged, about as long as fifth tergite, with straight posterior margin, mainly smooth. Ovipositor sheath shorter longer than metasoma.

\section{Diagnosis}

This genus differs from the remaining Rhaconotini genera by the following combination of characters: 1) scapus of antenna is with distinct apical fringe; 2) first flagellar segment of antenna shorter than second one; 3 ) second radiomedial vein ( $\mathrm{r}-\mathrm{m}$ ) and second radiomedial (submarginal) cell of fore wing absent; 4) second metasomal suture absent; 5) third-fifth metasomal tergites with distinct, strongly concave and crenulate furrows.

\section{Composition}

Grangerdoryctes niger (Granger, 1949).

\section{Hosts}

Unknown.

\section{Distribution}

Afrotropical region.

Grangerdoryctes niger (Granger, 1949)

Figs $12-13$

Aivalykus niger Granger, 1949: 145.

Aivalykus niger - Shenefelt \& Marsh 1976: 1353.

Grangerdoryctes niger - Belokobylskij 2005: 208. — Yu et al. 2016. — Jasso-Martínez et al. 2019: 167.

\section{Material examined}

\section{Holotype}

MADAGASCAR • ; "Madagascar, Bekily, reg. sud de l'ile", "Museum Paris, X.38, A. Seyrig", "25", "Type", "Holotypus Aivalykus niger Granger 1949", "Grangerdoryctes niger (Granger), det. Belokobylskij, 2002"; MNHN.

\section{Distribution}

Madagascar.

\section{Remarks}

The complete description of a single currently known specimen (holotype) of this species was published by Belokobylskij (2005). We have not examined any additional specimen of this species for description of possible morphological variation in G. niger. 


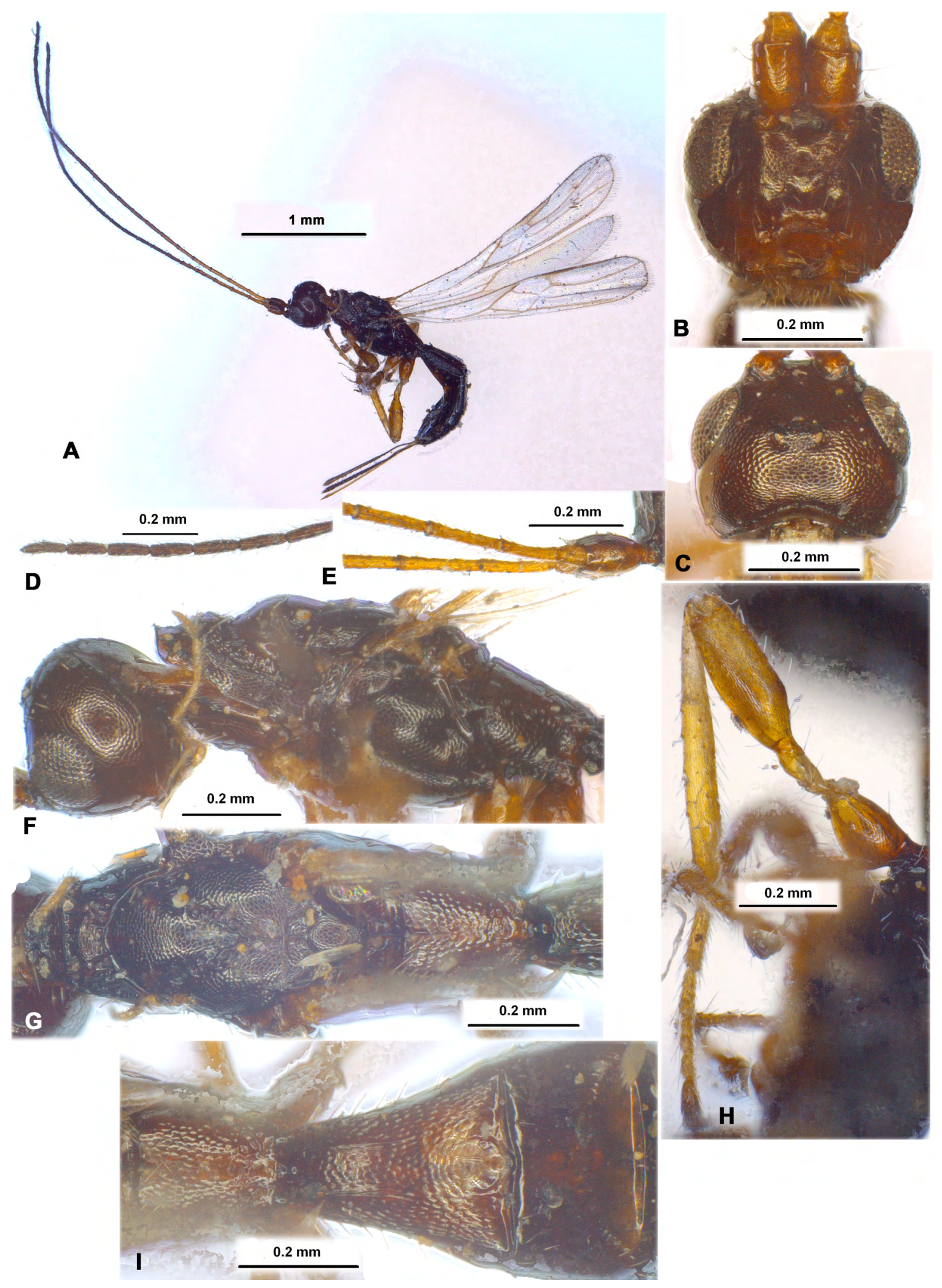

Fig. 12. Grangerdoryctes niger (Granger, 1949), $q$, holotype, MNHN. A. Habitus, lateral view. B. Head, front view. C. Head, dorsal view. D. Apical segments of antenna. E. Basal segments of antenna. F. Head and mesosoma, lateral view. G. Mesosoma, dorsal view. H. Hind leg. I. Propodeum and first to second metasomal tergites, dorsal view. 

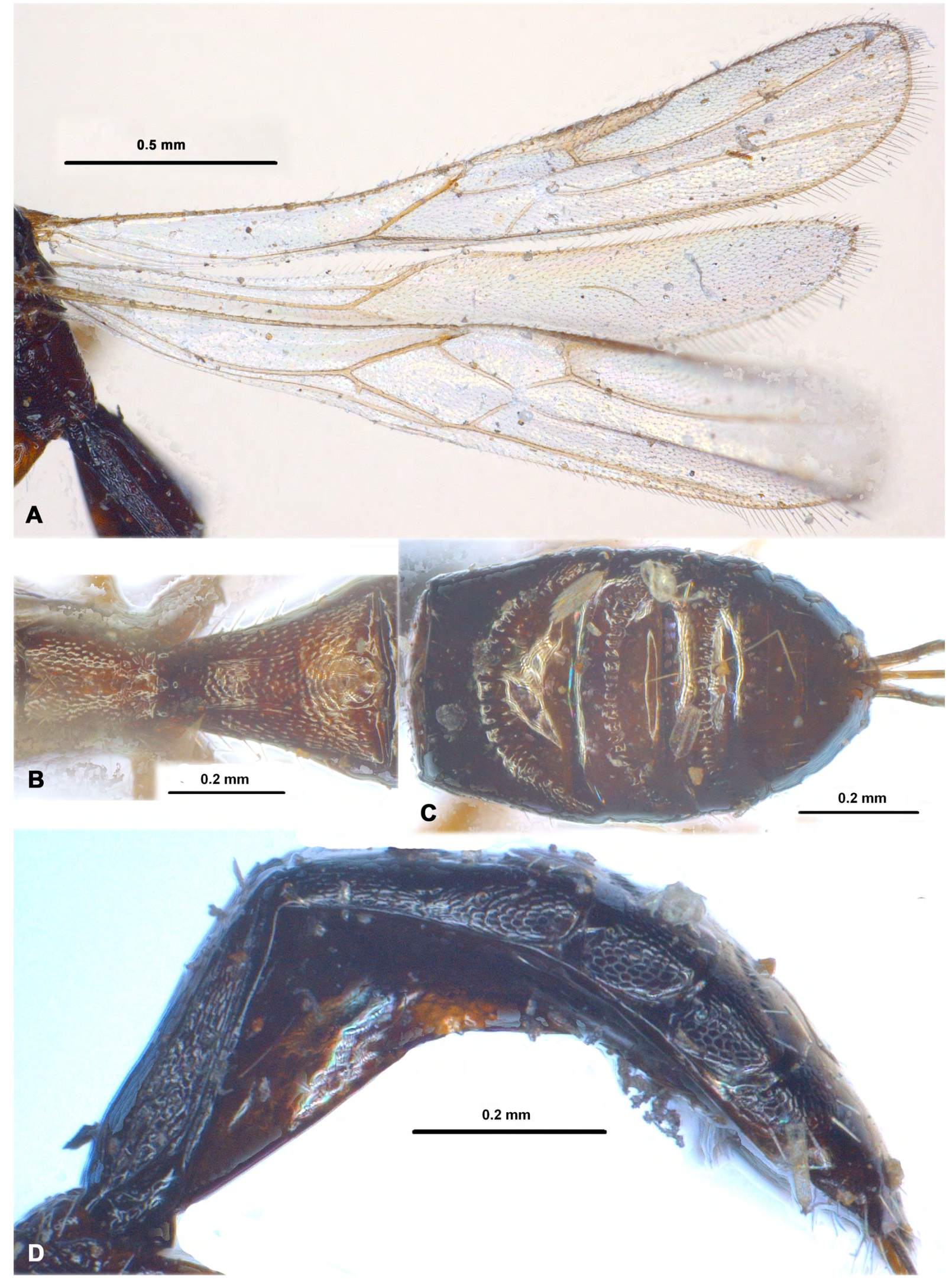

Fig. 13. Grangerdoryctes niger (Granger, 1949), $q$, holotype, MNHN. A. Wings. B. Propodeum and first metasomal, dorsal view. C. Metasoma (without first tergite), dorsal view. D. Metasoma, lateral view. 
Genus Ipodoryctes Granger, 1949

Ipodoryctes Granger, 1949: 106.

Ipodoryctes - Shenefelt \& Marsh 1976: 1267. — Belokobylskij 1994b: 129; 2001: 135; 2019: 36.Belokobylskij \& Maeto 2009: 284. — Tang et al. 2011: 1. — Yu et al. 2016. — Jasso-Martínez et al. 2019: 167. — Belokobylskij et al. 2019: 266.

\section{Type species}

Ipodoryctes anticestriatus Granger, 1949, by original designation.

\section{Description (Figs 14-21)}

Head. Head more or less transverse (dorsal view). Ocelli small or medium-sized, arranged in more or less obtuse triangle. Frons weakly convex or almost flat. Eyes glabrous. Occipital carina distinct, complete dorsally, obliterated below on distinct distance. Malar suture indistinct. Clypeal suture complete, but upper shallow. Hypoclypeal depression medium size, suboval or round. Face with two fine submedian elongate-oval depressions above clypeal suture. Postgenal bridge present, but narrow. Palps rather long; maxillary palps 6-segmented, its sixth (apical) segment about as long as fifth segment; labial palps 4-segmented, its third segment not shortened. Scapus of antenna wide and rather long, without apical lobe and basal constriction, its ventral margin (lateral view) shorter than dorsal margin. First flagellar segment not thickened, subcylindrical, longer than second segment.

Mesosoma. Mesosoma not depressed, rather long. Neck of prothorax short. Pronotum weakly convex dorsally (lateral view), pronotal carina rather fine, situated submedially on pronotum; pronope absent. Propleural dorsoposterior flange distinct and rather narrow. Mesonotum high and roundly elevated above pronotum. Median lobe of mesonotum weakly protruding forward, without anterolateral corners, often with distinct, usually incomplete median furrow. Notauli complete, rather wide, deep in anterior half and rather shallow in posterior half. Prescutellar depression usually shallow, with distinct median and often several lateral carinae, weakly curved on posterior margin. Lateral longitudinal wing-like flanges on level of prescutellar depression very low. Scuto-scutellar suture distinct and complete. Scutellum convex, often with fine lateral carinae. Mesopleural pit distinct, narrow, rather long, connected with mesopleural furrow by distinct furrow. Sternaulus (precoxal sulcus) rather shallow, narrow, long, curved. Prepectal carina distinct and complete. Prepectus usually with two strong longitudinal lateral carinae. Postpectal carina absent. Metanotum (lateral view) almost without median tooth. Metapleural flange rather short, wide, rounded apically. Propodeum often with rather distinct basolateral areas delineated by distinct carinae; lateral tubercles very weak; propodeal bridge absent. Propodeal spiracles small and round.

Wings. Pterostigma of fore wing wide and long. Radial vein (r) arising from middle of pterostigma. Radial (marginal) cell not shortened. Both radiomedial veins (2-SR, r-m) present. Second radiomedial (submarginal) cell long, rather wide, not widened towards apex. Recurrent vein (m-cu) more or less distinctly postfurcal. Discoidal (discal) cell petiolate anteriorly, petiole (1-SR) short. Mediocubital vein $(\mathrm{M}+\mathrm{CU} 1)$ very weakly curved to anal vein (2-1A) in posterior half. Nervulus (cu-a) distinctly postfurcal. Parallel vein (CU1a) often arising from or behind (from anterior 0.3) middle of distal margin of brachial (subdiscal) cell, but in several cases interstitial. Brachial (subdiscal) cell distally closed by more or less oblique brachial (CU1b) vein. Transverse anal veins (2A, a) absent. Hind wing with three hamuli. Radial vein (SR) arising from costal vein (2-SC+R) distant from basal vein (1r-m). Radial (marginal) cell almost parallel-sided, without additional transverse vein (r). Medial (basal) cell wide, distinctly widened towards apex, about 0.5 times as long as hind wing. Nervellus (cu-a) present. Submedial (subbasal) cell present and short. First abscissa of mediocubital vein $(\mathrm{M}+\mathrm{CU})$ distinctly shorter than second abscissa 
(1-M). Recurrent vein (m-cu) long, sclerotised at least partly and pigmented, distinctly oblique toward base of wing.

Legs. Fore and middle tibiae with rather sparse and thick spines arranged in almost single line. Middle tarsal segments long and weakly thickened. Hind coxa medium-sized, with distinct basoventral corner and usually with tubercle. All femora with low or very low and wide dorsal protuberances. Hind femur thickened, elongate-oval. Inner spurs of hind tibia rather short, straight, shortly setose, about 0.2 times as long as hind basitarsus. Hind basitarsus usually with narrow ventral carina, often about 0.7 times as long as second-fifth segments combined. Claws short, simple, robust, usually distinctly widened basally.

Metasoma. First tergite not petiolate, medium length and wide. Acrosternite of first segment short, about 0.2 times as long as first tergite, not reaching spiracular segments. Dorsope of first tergite rather deep and not large; spiracular tubercles indistinct or weak, spiracle situated in basal 0.3 of tergite; dorsal carinae present only basally or almost entirely, subbasally not united by additional transverse carina. Second tergite always with rather distinct semi-oval or subtransverse basal area delineated by rather distinct and usually curved furrow or only different type of sculpture; with distinct, more or less wide and curved or rarely almost straight posterior furrow delineated wide or narrow sublenticular apical area. Second suture distinct, rather deep and wide, evenly curved, without lateral bends. Fourth and usually fifth tergites with basal shallow, crenulate and curved furrows. Second-sixth tergites with separate laterotergites. Sixth tergite enlarged or not enlarged, almost equal to fifth segment (till subbasal transverse furrow) or often shorter than it, coarsely sclerotised, usually almost entirely sculptured, but sometimes finely sculptured till partly or entirely smooth; its posterior margin weakly convex, usually without median excision. Ovipositor apically with two obtuse and distinct or very low dorsal nodes. Ovipositor sheath often long, longer than metasoma.

\section{Diagnosis}

Ipodoryctes Granger, 1949 is morphologically similar to Bathycentor Saussure, 1892. The differences between these two genera are mentioned in the redescription of Bathycentor and in the above key.

\section{Composition}

This genus currently consists of two subgenera, Afroipodoryctes subgen. nov. and Ipodoryctes s.str. The following species are belonged to the nominative subgenus: Ipodoryctes (Ipodoryctes) albiflagellus (Shi \& Chen, 2004) comb. nov. (OR); I. (I.) alutaceus Granger, 1949 (AF); I. (I.) annulicornis Belokobylskij, 1994 (OR); I. (I.) anticestriatus Granger, 1949 (AF); I. (I.) brachiceps (Belokobylskij \& Maeto, 2009) comb. nov. (OR); I. (I.) elongatus Granger, 1949 (AF); I. (I.) formosanus (Watanabe, 1934) (Rhaconotus carolinensis Watanabe, 1945) (PA, OR, PC); I. (I.) forticarinatus (Chen \& Shi, 2004) comb. nov. (OR); I. (I.) giganteus (Granger, 1949) comb. nov. (AF); I. (I.) interstitialis Granger, 1949; I. (I.) longus (Shi \& Chen, 2004) comb. nov. (OR); I. (I.) maculistigma (Chen \& Shi, 2004) comb. nov. (OR); I. (I.) niger Granger, 1949 (AF); I. (I.) nitidus Belokobylskij, 2001 (OR); I. (I.) pallidipes Granger, 1949 (AF); I. (I.) pilosus (Granger, 1949) comb. nov. (AF); I. (I.) puber (Belokobylskij, 2001) comb. nov. (OR); I. (I.) rufiventris (Chen \& Shi, 2004) comb. nov. (OR); I. (I.) rugosiscutum Belokobylskij, 1994 (OR); I. (I.) ryukyuensis Belokobylskij, 2001 (OR); I. (I.) signatus (Belokobylskij, 2001) comb. nov. (OR, PA); I. (I.) signipennis (Walker, 1860) (Rhaconotus flavistigmus Belokobylskij, 1983) (PA, OR, NA); I. (I.) sonaicus (Belokobylskij \& Maeto, 2009) comb. nov. (OR); I. (I.) tamdaoensis Belokobylskij, 1994 (OR); I. (I.) temporalis Belokobylskij, 2001 (OR); I. (I.) vagrans (Bridwell, 1920) (PA, OR, PC); I. (I.) watanabei (Belokobylskij \& Maeto, 2009) comb. nov. (OR); I. (I.) roslinensis (Lal, 1939) comb. nov. (OR). 


\section{Hosts}

Lepidoptera: Phragmataecia sp. (Cossidae); Bissetia steniella (Hampson, 1899), Chilo kanra (Fletcher, 1928), Ch. partellus (Swinhoe, 1885), Ch. saccharifagus (Bojer, 1856); Eoreuma loftini (Dyar, 1917), Scirpophaga sp., S. excerptalis (Walker, 1863) (Crambidae).

\section{Distribution}

Afrotropical, Oriental and Palaearctic regions.

Ipodoryctes anticestriatus (Granger, 1949)

Figs $14-15$

Ipodoryctes anticestriatus Granger, 1949: 112.

Ipodoryctes anticestriatus - Shenefelt \& Marsh 1976: 1314. — Yu et al. 2016. — Jasso-Martínez et al. 2019: 167.

\section{Material examined}

Holotype

MADAGASCAR • क; "Madagascar, Bekily, reg sud de 1'ile", "Museum Paris, I - 37, A. Seyrig", “Type", “+ Ipodoryctes anticestriatus Granger, C. van Achterberg, 1983, Holotype”; MNHN.

\section{Description}

\section{Female}

Measurements. Body length $5.4 \mathrm{~mm}$, fore wing length $3.8 \mathrm{~mm}$.

HEAD. Width 1.45 times its median length, 1.15 times width of mesoscutum. Head behind eyes (dorsal view) distinctly roundly narrowed. Transverse diameter of eye (dorsal view) about 2.0 times as long as temple. Ocelli rather small, arranged in triangle with base 1.1 times its sides, situated near median line of eyes. POL almost equal to OD, 0.6 times OOL. Eye with distinct emargination opposite antennal sockets, 1.25 times as high as broad. Malar space 0.3 times height of eye and 0.8 times basal width of mandible. Face width 0.75 times height of eye and 1.15 times height of face and clypeus combined. Clypeus convex. Hypoclypeal depression oval, its width 0.9 times distance from edge of depression to eye and 0.45 times width of face. Hypostomal flange narrow. Palps long, length of maxillary palps 1.1 times head height (without mandible). Antennae slender, weakly setiform, more than 34-segmented. Scapus 1.5 times as long as its maximum width. First flagellar segment 4.3 times as long as its apical width, 1.25 times as long as second segment. Subapical segments about 3.5 times as long as width.

Mesosoma. Length 2.0 times its height. Median lobe of mesoscutum convex, distinctly protruding forward and rounded. Mesoscutum as long as maximum width. Notauli deep anteriorly and shallow posteriorly, wide and coarsely rugose-crenulate. Prescutellar depression with median and four lateral carinae, finely rugulose-reticulate, 0.4 times as long as scutellum. Scutellum weakly convex, as long as its maximum anterior width. Subalar depression striate, with additional rugulosity and granulation. Sternaulus (precoxal furrow) shallow, running along anterior 0.6 of lower part of mesopleuron, finely reticulate. Metapleural lobe without dense pubescence.

WiNGs. Fore wing 3.3 times as long as its maximum width. Pterostigma 4.0 times as long as wide. Metacarpus (1-R1) 1.2 times as long as pterostigma. First (r) and second (3-SR) radial abscissae forming obtuse angle. Second radial abscissa (3-SR) 3.8 times as long as first abscissa (r), about 0.9 times as long as third abscissa (SR1), 1.8 times as long as first radiomedial vein (2-SR). Second radiomedial (submarginal) cell long, 3.4 times as long as its maximum width, 1.4 times as long as the rather wide 


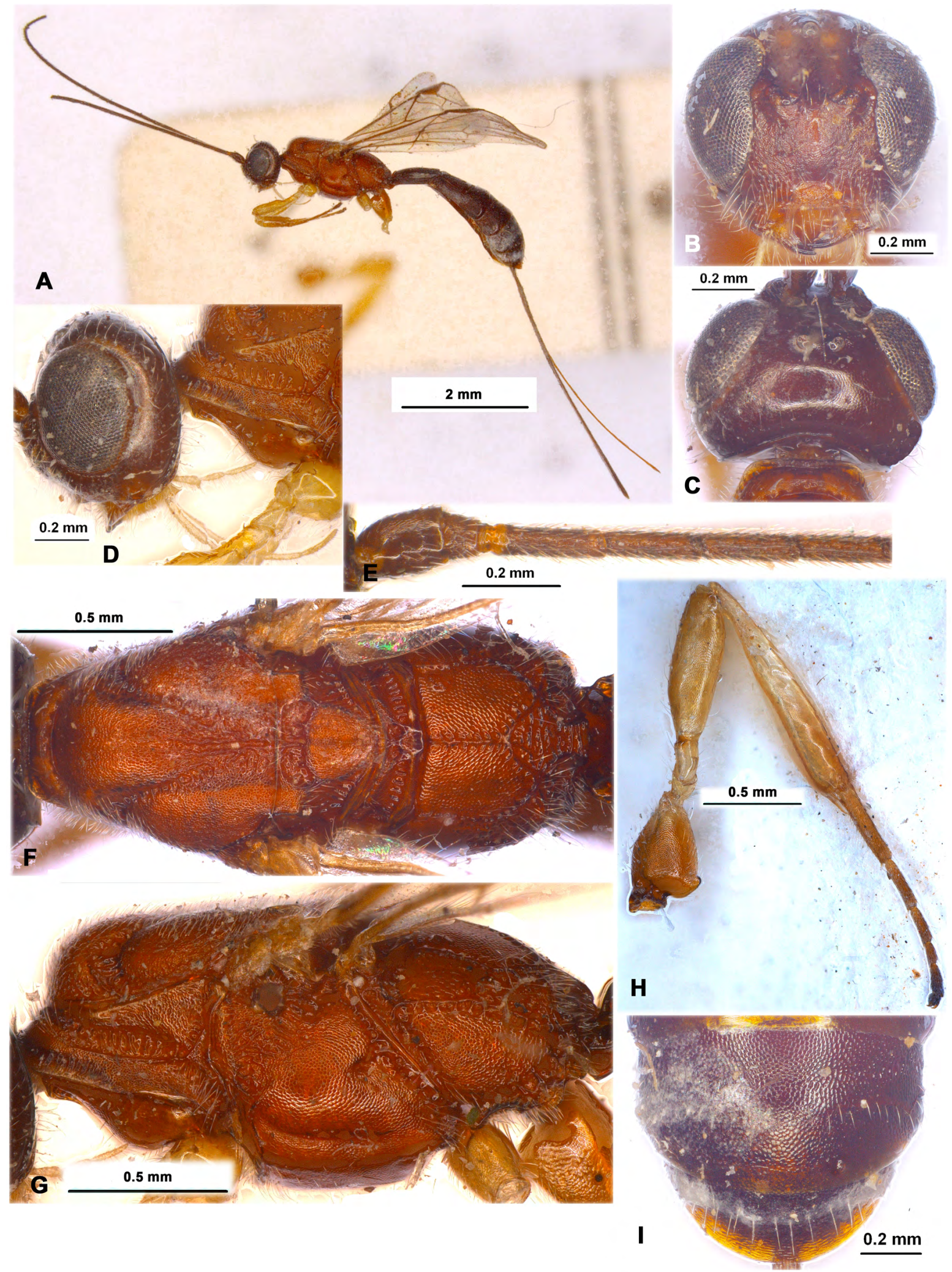

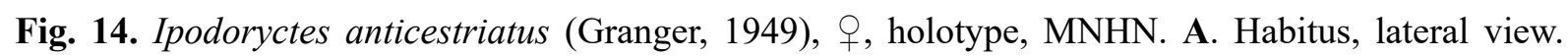
B. Head, front view. C. Head, dorsal view. D. Head, lateral view. E. Basal segments of antenna. F. Mesosoma, dorsal view. G. Mesosoma, lateral view. H. Hind leg. I. apical segments of metasoma, dorsal view. 
BELOKOBYLSKIJ S.A. \& ZALDÍVAR-RIVERÓN A., Reclassification of the Rhaconotini
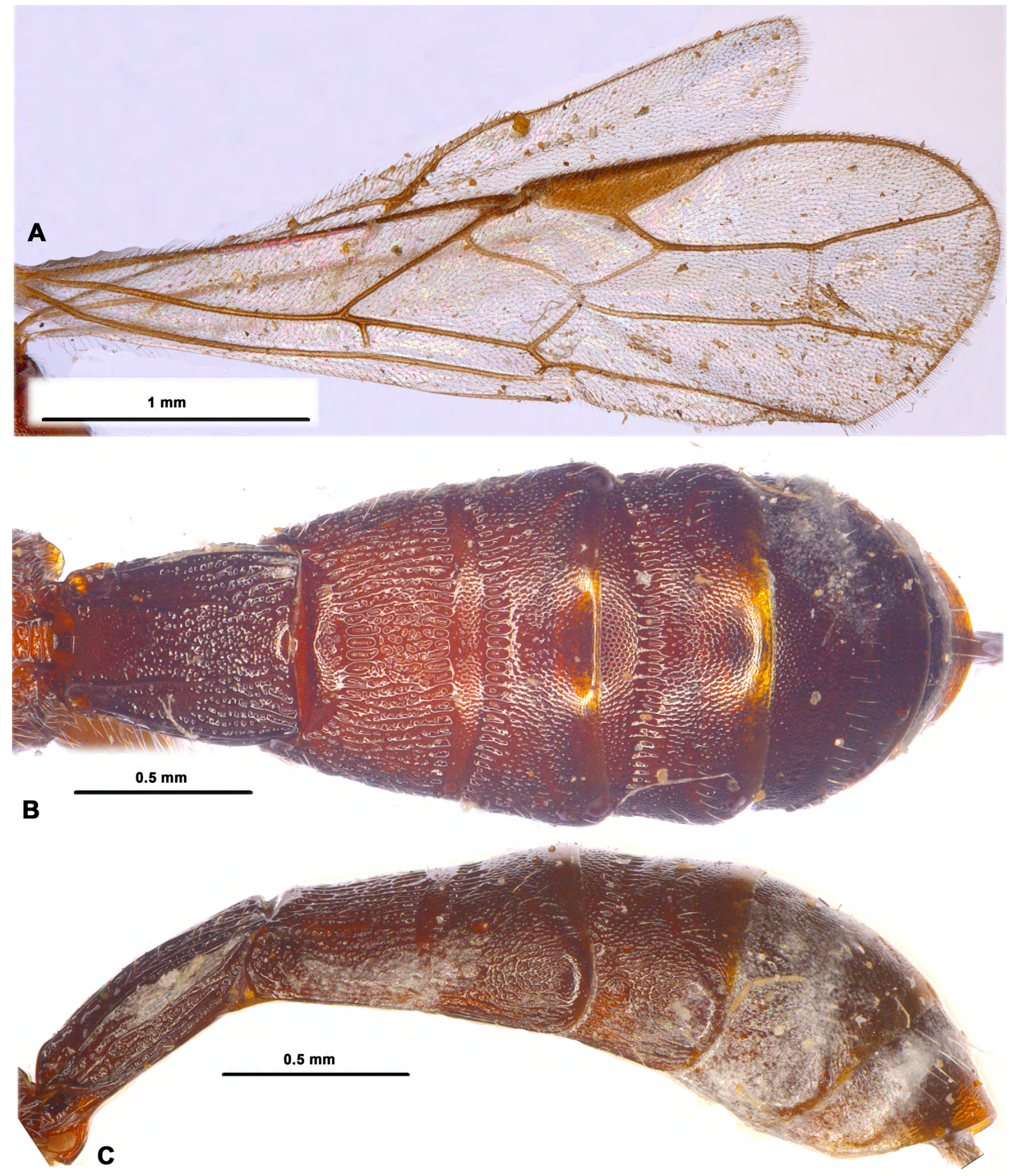

Fig. 15. Ipodoryctes anticestriatus (Granger, 1949), + , holotype, MNHN. A. Wings. B. Metasoma, dorsal view. C. Metasoma, lateral view. 
brachial (subdiscal) cell. First medial abscissa (1-SR+M) distinctly sinuate. Distance from nervulus (cua) to basal (1-M) vein 0.7 times as long as nervulus (cu-a) length. Hind wing 4.8 times as long as wide. First mediocubital abscissa $(\mathrm{M}+\mathrm{CU}) 0.75$ times as long as second abscissa (1-M). Recurrent vein (m-cu) long, weakly curved, antefurcal, mostly sclerotised.

Legs. Hind coxa 1.3 times as long as wide, 0.75 times as long as propodeum. Hind femur 3.0 times as long as wide. Hind tarsus almost as long as hind tibia. Basitarsus of hind tibia 0.5 times as long as second-fifth segments combined. Second tarsal segment 0.65 times as long as basitarsus, 1.6 times as long as fifth segment (without pretarsus).

Metasoma. Metasoma 1.2 times as long as head and mesosoma combined. First tergite rather weakly and curvedly widened from base to subapex, then weakly narrowed towards apex. Maximum width of first tergite 1.75 times its minimum width; length about 1.5 times its apical width. Median length of second tergite (with both areas) 0.8 times its basal width, 1.8 times as long as third tergite. Basal area of second tergite 0.4 times as long as rest part of tergite; posterior (apical) area 0.3 times as long as rest part of tergite. Six tergite 0.9 times as long as fifth segment (measured from fine transverse basal furrow) and equal to fourth segment, its posterior margin very weakly incurved medially. Ovipositor sheath long, 0.8 times as long as body, 2.2 times as long as mesosoma, 1.5 times as long as metasoma, 1.1 times as long as fore wing.

SCULPTURE AND PUBESCENCE. Vertex very finally and densely coriaceous, partly posteriorly almost smooth; frons densely and finely granulate, almost smooth narrowly medially. Face densely granulate, with additional sparse punctation and laterally with fine rugulosity, almost smooth narrow medially. Temple very finely coriaceous, smooth below. Mesoscutum densely granulate, coarsely rugose on wide area in its medioposterior half. Scutellum densely and finely granulate. Mesopleuron densely and rather finely areolate-coriaceous, striate-granulate in upper 0.25-0.30. Propodeum with large and densely granulate basolateral areas and short rugae along carinae; areola coarsely rugose, wide and short, almost as long as its maximum width; basal carina1.6 times as long as side of anterior fork of areola. Hind coxae almost entirely and densely granulate, without dorsal striation. First tergite rugose-striate, with dense granulation in mediobasal third. Second tergite distinctly striate and with dense reticulation between striae, its basal and apical areas densely and finely granulate-coriaceous. Third and fourth tergites densely granulate-reticulate, smooth apically. Fifth tergite finely and densely reticulate-coriaceous, smooth apically. Sixth tergite finely reticulate basally, partly interruptedly transverse striate in apical half. Vertex with sparse, short and semi-erect white setae. Mesoscutum entirely with very dense, short and almost erect white setae. Hind tibia dorsally with rather short, sparse and semi-erect pale setae.

Colour. Head dark reddish brown, face reddish brown, clypeus and mandible yellowish brown. Mesosoma light reddish brown. Metasoma reddish brown, darker basally and apically. Antenna dark brown to black, two basal segments reddish brown, subapical segments black. Palps entirely yellow. Legs yellow, tibiae and tarsi brownish yellow, hind coxa light reddish brown, hind tibia pale basally. Ovipositor sheath entirely black. Fore wing very faintly infuscate. Pterostigma entirely brown.

\section{Male}

Unknown.

\section{Distribution}

Madagascar. 


\section{Subgenus Afroipodoryctes subgen. nov. urn:lsid:zoobank.org:act:36CDE5C4-B0FF-4939-BA6C-1C985F9F8846}

\section{Type species}

Ipodoryctes insignis Granger, 1949.

\section{Etymology}

From combination of the words "Africa" and the generic name "Ipodoryctes", since members of this subgenus were only recorded in the African continent.

\section{Description (Figs 16-21)}

HEAD. Head transverse. Ocelli arranged in weakly obtuse triangle with base weakly larger than its sides. Eyes glabrous. Occipital carina incomplete, obliterate below and not joining ventrally with hypostomal carina at short distance. Malar suture absent. Hypoclypeal depression medium size, subround. Postgenal bridge very narrow. Scapus of antenna more or less wide. First flagellar segment subcylindrical, almost straight, not shorter than second segment. Apical segment apically acuminate.

Mesosoma. Mesosoma not depressed and relatively long. Pronotum dorsally weakly convex, with more or less fine pronotal carina. Mesonotum more or less highly and gently roundly elevated above pronotum; mainly smooth and glabrous. Notauli distinct, almost incomplete, smoothed posteriorly, smooth or partly finely crenulate. Prescutellar depression rather long, with several carinae. Metanotum with short and more or less pointed median tooth (lateral view). Sternaulus (precoxal furrow) rather deep, long, running along anterior $0.6-0.8$ of lower part of mesopleuron. Propodeum with basolateral areas and areola distinctly delineated by carinae; lateral tubercles short.

WINGS. Wings not shortened. Pterostigma of fore wing wide. Radial vein of fore wing arising almost from middle of pterostigma. Radial (marginal) cell not shortened. Second radiomedial (submarginal) cell long. Recurrent vein (m-cu) distinctly postfurcal. Nervulus (cu-a) postfurcal. Parallel vein (CU1a) interstitial or not interstitial and arising from anterior 0.3 of distal margin of brachial (subdiscal) cell. Brachial (subdiscal) cell subvertically closed postero-apically before recurrent vein (m-cu) and with antero-posteriorly corner. In hind wing, first abscissa of mediocubital vein $(\mathrm{M}+\mathrm{CU})$ 0.4-0.5 times as long as second abscissa (1-M).

LEGS. Hind coxa without or with small basoventral tubercle. Hind femur rather wide, almost without dorsal protuberance. Hind basitarsus 0.7 times as long as second-fifth segments combined.

Metasoma. First tergite not petiolate, rather long and widened toward apex. Second tergite with narrow basal area delineated by rather distinct furrow; with lenticular apical area distinctly delineated anteriorly by deep crenulate furrow and posteriorly by second suture. Second suture deep, complete and regularly curved. Sixth tergite not or weakly enlarged, not longer than previous tergite, smooth, more or less covered following apical segments, posteriorly evenly curved, without emargination. Ovipositor sheath not longer than metasoma.

\section{Diagnosis}

This new subgenus differs from Ipodoryctes s.str. in having the hind coxa without or sometimes with indistinct basoventral tubercle; pro- and mesothorax almost entirely smooth; propodeum with areola delineated by distinct carinae, notauli smooth or almost smooth. 


\section{Composition}

Ipodoryctes (Afroipodoryctes) insignis (Granger, 1949) (AF); I. (A.) reunionus sp. nov.; I. (A.) saintphillipensis sp. nov.

\section{Distribution}

Afrotropical region.

\section{Key to species of Ipodoryctes (Afroipodoryctes)}

1. Mesoscutum entirely densely and distinctly granulate and almost entirely covered by short pale setae (Figs 20F-G). Body length 2.1-3.2 mm. La Reunion I. (A.) saintphillipensis sp. nov.

- Mesoscutum entirely smooth and mainly glabrous (Figs 16C-D; $18 \mathrm{H}-\mathrm{I}$ ) 2

2. Areola of propodeum narrow (Fig. 16C). Brachial (subdiscal) cell of fore wing apically closed before recurrent vein (m-cu); parallel vein (CU1a) of fore wing interstitial (Fig. 17A). Hind femur yellow basally and dark reddish brown in posterior 0.6 (Fig. 16CB. First metasomal tergite narrow and long, its length 1.6 times apical width. Second metasomal suture evenly curved (Fig. 17B). Body length $4.0 \mathrm{~mm}$. Madagascar

I. (A.) insignis (Granger, 1949)

- Areola of propodeum wide (Fig. 18H). Brachial (subdiscal) cell of fore wing apically closed behind recurrent vein $(\mathrm{m}-\mathrm{cu})$; parallel vein (CU1a) of fore wing often arising from anterior $0.2-0.3$ of distal margin of brachial (subdiscal) cell (Fig. 19A). Hind femur almost entirely pale brown to yellow (Fig. 18J). First metasomal tergite wide and short, its length 1.0-1.2 times its apical width. Second metasomal suture sinuate (Figs 19B, D). Body length 3.1-4.3 mm. La Reunion

I. (A.) reunionus sp. nov.

\section{Ipodoryctes (Afroipodoryctes) insignis Granger, 1949}

Figs 16-17

Ipodoryctes insignis Granger, 1949: 109.

Ipodoryctes insignis - Shenefelt \& Marsh 1976: 1314. — Yu et al. 2016.

\section{Material examined}

\section{Holotype}

MADAGASCAR • +; "Madagascar, Perinet, Foret cote est", "Museum Paris, 3-32, A. Seyrig", "36", "Type", "Ipodoryctes insignis Granger" (head, all fore legs, and single middle and hind legs missing); MNHM.

\section{Description}

\section{Female}

MEasurements. Body length (according to original description) $4.0 \mathrm{~mm}$; fore wing length $3.6 \mathrm{~mm}$.

HEAD. Head and antennae missing; but according to original description (Granger 1949: 109): head transverse, strongly narrowed behind eyes. Antennae 36-segmented, 1.5 times as long as body.

Mesosoma. Length 2 times its height. Pronotum almost straight dorsally (lateral view). Pronotal carina distinct, situated submedially. Mesoscutum rather highly and roundly elevated above pronotum. Notauli deep, complete, smooth. Median lobe of mesoscutum without median depression. Prescutellar depression rather shallow, short, with five carinae, rugulose, 0.3 times as long as scutellum. Scutellum weakly convex. Sternaulus (precoxal furrow) shallow, straight, mostly smooth, finely crenulate only posteriorly, running along almost entire lower part of mesopleuron, connected with prepectal carina anteriorly. 
Prepectal carina below without widened lobes opposite fore coxae. Subalar depression shallow, narrow, densely striate. Metanotum with long pointed tooth. Metapleural lobe long and wide.

WiNGS. Length of fore wing 4.0 times its maximum width. Radial (marginal) cell not shortened. Metacarpus (1-R1) 1.2 times as long as pterostigma. Pterostigma 3.7 times as long as wide. Radial vein (r) arising almost from middle of pterostigma. First radial abscissa (r) forming distinct obtuse angle with second abscissa (3-SR). Second radial abscissa (3-SR) 5.3 times as long as first abscissa (r), 0.8 times as long as third abscissa (SR1), 2.2 times as long as first radiomedial vein (2-SR). Second radiomedial (submarginal) cell not widened distally, its length 3.5 times maximum width, 1.2 times length of rather narrow brachial (subdiscal) cell. First abscissa of medial vein (1-SR+M) distinctly sinuate. Recurrent vein $(\mathrm{m}-\mathrm{cu})$ distinctly postfurcal, 3.5 times as long as second abscissa of medial vein $(2-\mathrm{SR}+\mathrm{M})$. Mediocubital vein (M+CU1) distinctly curved to anal vein (2-1A) in distal half. Distance from nervulus (cu-a) to basal (1-M) vein 0.6 times nervulus (cu-a) length. Brachial (subdiscal) cell apically gently and roundly closed distinctly before level of recurrent vein (m-cu); posterior bulla indistinct; posterior abscissa of anal vein (2-1A) (behind brachial vein (CU1b)) absent. Hind wing about 5.0 times as long as wide. First abscissa of mediocubital vein $(\mathrm{M}+\mathrm{CU}) 0.6$ times as long as second abscissa (1-M). Recurrent vein (m-cu) strongly unsclerotised, antefurcal. Medial (basal) cell rather narrow, distinctly widened distally, 0.45 times as long as wing.

LEGS. Hind coxa without basoventral tubercle. Hind and middle femora without dorsal protuberances. Length of hind femur 3.0 times its maximum width. Hind tibia thickened. Tarsal segments of hind leg mostly missing, basitarsus short.

Metasoma. Length 1.5 times as long as mesosoma, with six visible tergites, but sixth tergites not strongly enlarged. First tergite long, regularly and linearly widened toward apex, with lateral keels, without spiracular tubercles. Apical width of first tergite 1.8 times its basal width; its length 1.6 times apical width, 1.4 times length of propodeum. Second tergite with deep, distinctly curved transverse furrow and with distinct wide apical lenticular area, this area 0.6 times as long as rest part of tergite. Median length of second tergite 0.8 times its basal width, almost 2 times length of third tergite. Second suture deep and distinctly curved. Fifth tergite 1.2 times as long as fourth tergite, 1.5 times as long as third tergite, weakly shorter than sixth tergite. Posterior margins of fifth and sixth tergites almost straight, without median emargination and postero-ventral lobes. Ovipositor sheath 0.7 times as long as metasoma, as long as mesosoma, 0.45 times as long as fore wing.

SCULPTURE AND PUBESCENCE. Head (according to original description) smooth. Pro- and mesothorax mostly smooth; mesoscutum posteriorly with two straight carinae Propodeum almost entirely smooth, with median carina in basal half, basolateral areas long, about 0.7 times as long as propodeum; areola distinct, rather narrow, pentagonal, weakly rugulose. Hind legs very finely granulate, hind coxa almost smooth, without dorsal striation. First tergite entirely, second at most part (except smooth apical area) and base of third tergite distinctly striate, remainder parts of metasoma smooth, except narrowly striate bases of tergites. Mesoscutum with sparse long setae arranged along notauli and marginally. Hind tibia with erect sparse long setae dorsally; length of these setae 1.2-1.5 times maximum width of hind tibia.

Colour. Body reddish brown. Legs yellow, hind coxa and especially distal half of hind femur brown to dark brown. Fore wings evenly and distinctly infuscate. Pterostigma brown, yellow in basal half.

\section{Male}

Unknown.

\section{Distribution}

Madagascar. 


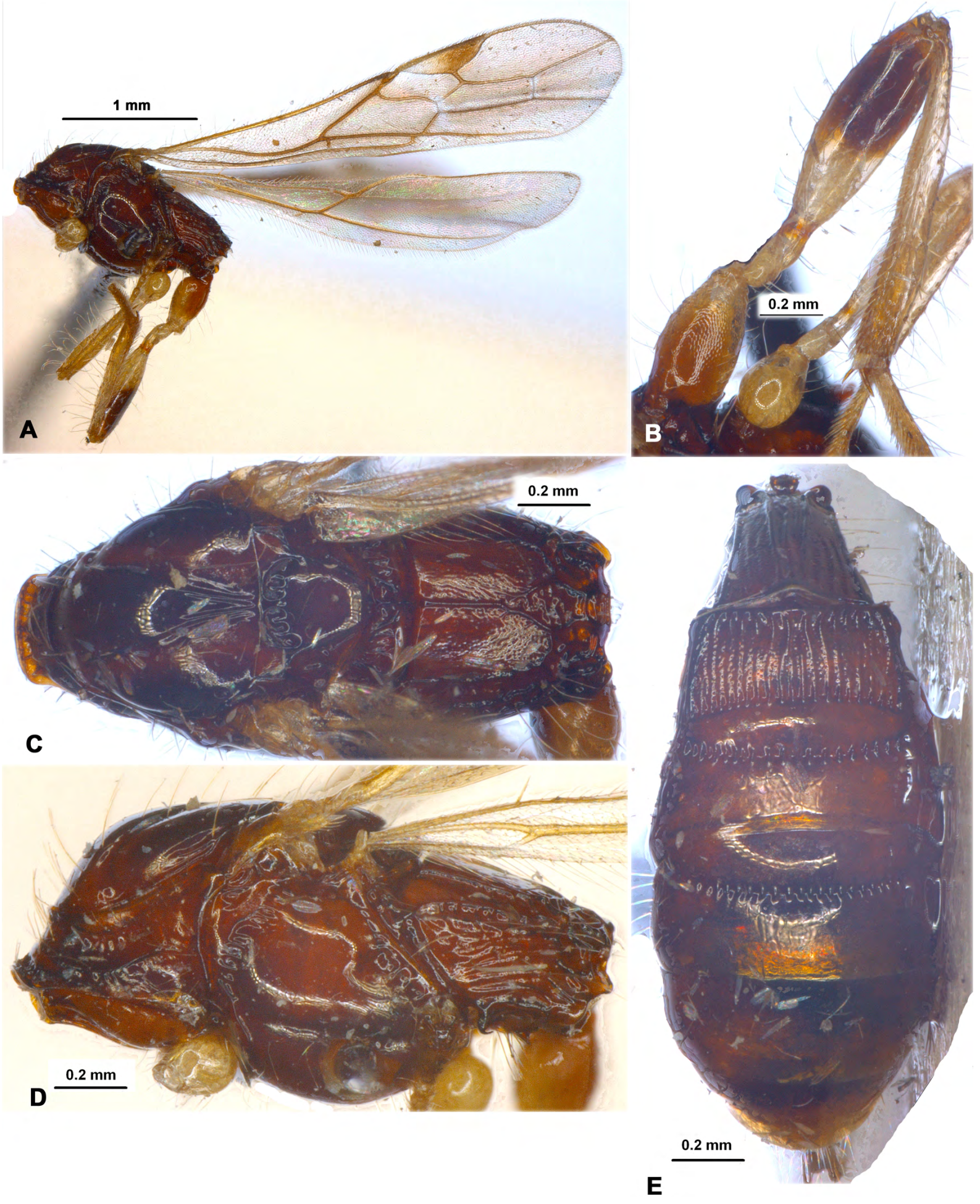

Fig. 16. Ipodoryctes (Afroipodoryctes) insignis (Granger, 1949),, , holotype, MNHM. A. Habitus, lateral view. B. Hind leg, lateral view. C. Mesosoma, dorsal view. D. Mesosoma, lateral view. E. Metasoma, dorsal view. 


\section{BELOKOBYLSKIJ S.A. \& ZALDÍVAR-RIVERÓN A., Reclassification of the Rhaconotini}
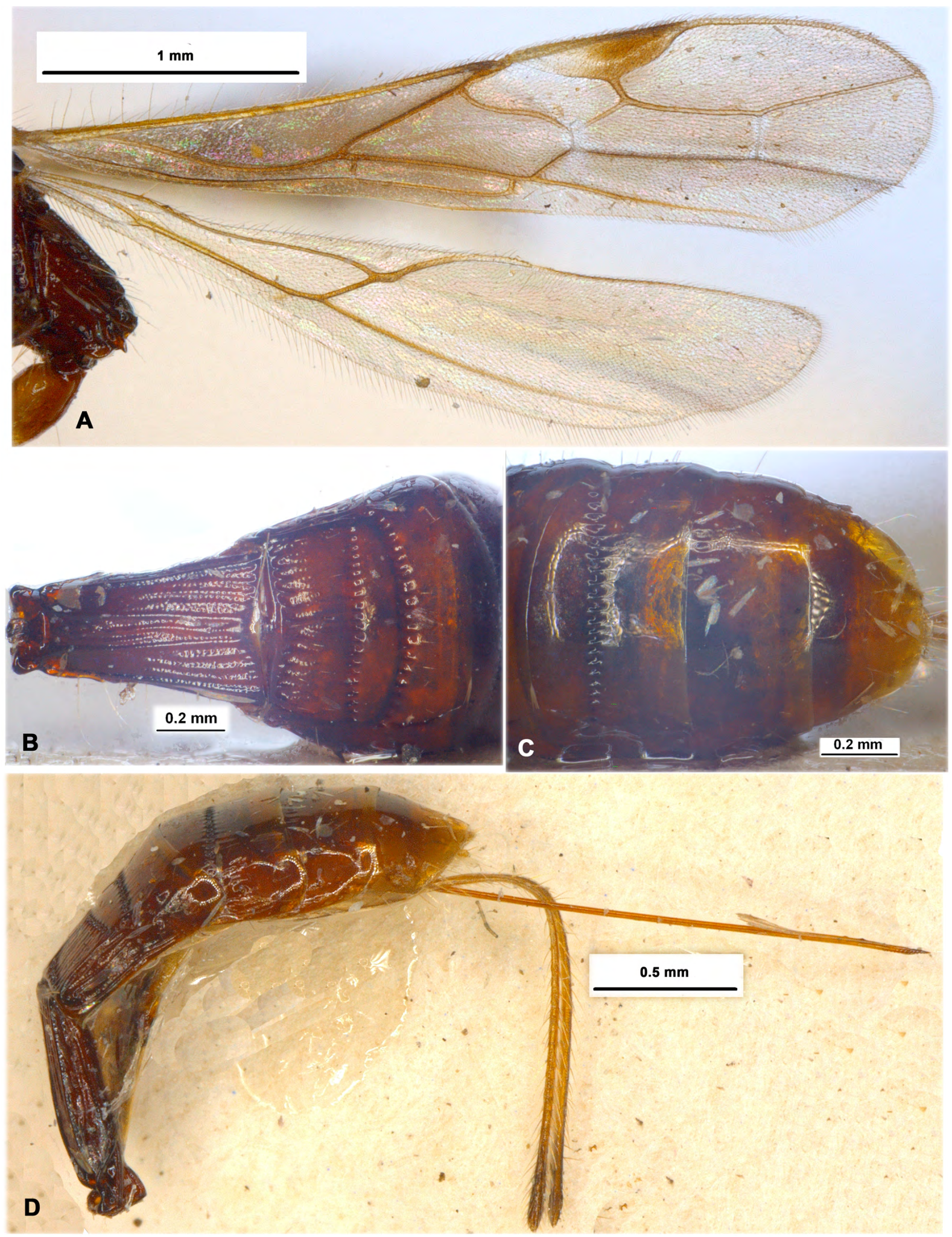

Fig. 17. Ipodoryctes (Afroipodoryctes) insignis (Granger, 1949),, , holotype, MNHM. A. Wings. B. First to third metasomal tergites, dorsal view. C. Apical metasomal tergites, dorsal view. D. Metasoma and ovipositor, lateral view. 
Ipodoryctes (Afroipodoryctes) reunionus sp. nov. urn:1sid:zoobank.org:act:424A57AA-E3B9-43DA-B76D-8CABA2B7CFD1

Figs $18-19$

\section{Etymology}

The name of this species refers to the Réunion Island, where the type material was collected.

\section{Material examined}

\section{Holotype}

REUNION • + ; "La Reunion, ML 221350 m, Saint Philippe", "Mare Longue, 25.IX/01.X.2001, Attie Marc leg", "forêt primaire, piege Malaise, au sol site 2", "RUS44 [= Jo932]"; MNHM.

\section{Paratypes}

REUNION • 1 \%; "La Reunion, PF 258780 m, Sainte Suzanne", "forêt Dugain, 25.XI/01.XII.2001, Attie Marc leg", "piege Malaise, au sol dans taillis de Psidium cattleianum"; MNHM • 1 ô; "La Reunion, A Sainte-Suzanne", "forêt Dugain, 780 m", "taillis de Psidium cattleianum, 16/22.II.2001", "piege Malaise, au sol, Attie Marc leg"; MNHM • 1 ठ઼; "La Reunion, ML 284350 m, Saint Philippe", "Mare Longue, 18/24.XI.2001, Attie Marc leg", "forêt primaire, piege Malaise, suspendu site 1"; ZISP.

\section{Description}

\section{Female}

MeAsuremEnTs. Body length 3.1-4.3 mm; fore wing length 2.6-3.3 mm.

HEAD. Head width 1.4-1.6 times its median length, 1.1 times width of mesoscutum. Head behind eyes (dorsal view) distinctly and roundly narrowed. Transverse diameter of eye 1.7-2.0 times as long as temple. Ocelli small, arranged in almost equilateral triangle; POL 1.2-1.6 times OD, 0.4-0.5 times OOL. Eye weakly emarginated opposite antennal sockets, $1.15-1.20$ times as high as broad. Malar space 0.3 times height of eye, $0.7-0.8$ times basal width of mandible. Face width 0.9 times eye height and 1.1-1.2 times height of face and clypeus combined. Upper margin of clypeus situated distinctly higher than lower level of eyes. Width of hypoclypeal depression about 1.1 times distance from depression to eye, 0.45 times width of face. Vertex convex. Head below eyes (front view) strongly and almost linearly or weakly roundly narrowed. Antennae slender, weakly filiform, 26-33-segmented, about 1.2 times as long as body. Scapus 1.4-1.6 times as long as its maximum width, 2.0-2.5 times length of pedicel. First flagellar segment 4.0-4.5 times as long as its apical width, about as long as second segment. Penultimate segment 4.0-4.5 times as long as its width, about 0.8 times as long as first segment, 0.8-0.9 times as long as apical segment.

Mesosoma. Length 2.1-2.2 times its height. Pronotal carina high, situated submedially. Mesoscutum (dorsal view) $0.8-0.9$ times as long as wide. Notauli distinct in anterior half, shallow or very shallow (in holotype) in posterior half, narrow, densely crenulate only in anterior half (holotype) or entirely. Median lobe of mesoscutum distinctly convex anteriorly. Prescutellar depression with distinct median and four lateral carinae, almost smooth or weakly rugulose on rest part, weakly curved along posterior margin, $0.30-0.35$ times as long as scutellum. Scutellum equal to or weakly longer than its maximum width. Subalar depression coarsely crenulate at narrow area. Sternaulus (precoxal furrow) entirely smooth. Prepectal carina below with low lobes opposite fore coxae. Metanotum dorso-medially with distinct two oblique lateral carinae. Propodeum weakly arcuately sloping backward (lateral view). Metapleural flange posteriorly with sparse long pubescence.

WINGS. Length of fore wing 3.5-3.6 times its maximum width. Pterostigma 4.0-4.5 times as long as wide. Metacarpus (1-R1) 1.2-1.3 times as long as pterostigma. Radial (marginal) cell 3.5-3.7 times as long as wide. Radial vein (r) arising from middle of pterostigma. First radial abscissa (r) forming obtuse 


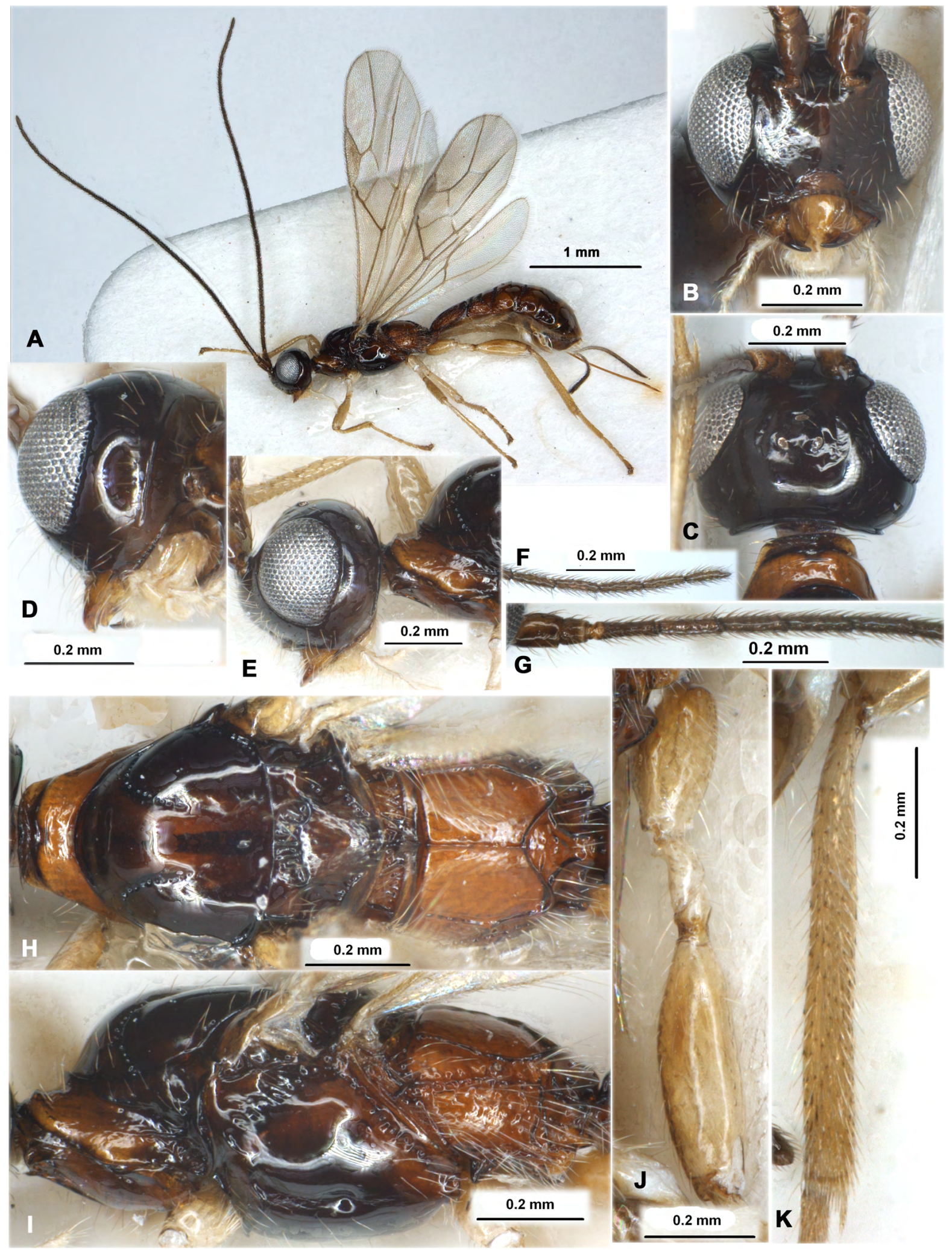

Fig. 18. Ipodoryctes (Afroipodoryctes) reunionus sp. nov., $\circ$, holotype, MNHM. A. Habitus, lateral view. B. Head, front view. C. Head, dorsal view. D. Head, latero-posterior view. E. Head, lateral view. F. Apical segments of antenna. G. Basal segments of antenna. H. Mesosoma, dorsal view. I. Mesosoma, dorsal view. J. Hind coxa and femur. K. Hind tibia. 

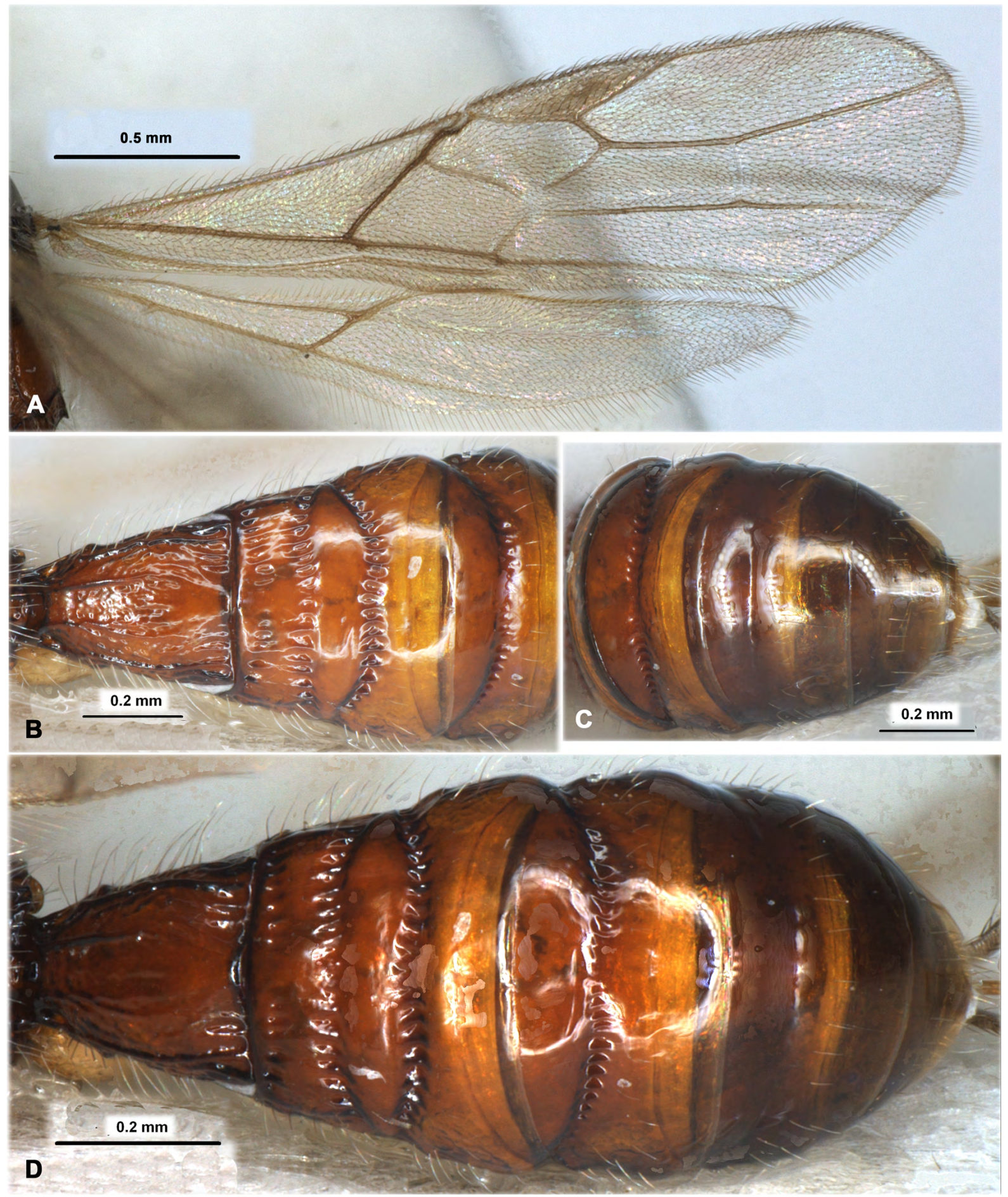

Fig. 19. Ipodoryctes (Afroipodoryctes) reunionus sp. nov.,, , holotype, MNHM. A. Wings. B. First to third metasomal tergites, dorsal view. C. Apical metasomal tergites, dorsal view. D. Metasoma, dorsal view. 
corner with second abscissa (3-SR). Second radial abscissa (3-SR) about 3.0 times as long as first abscissa (r), 0.6 times as long as the straight third abscissa (SR1), 1.4 times as long as second radiomedial vein $(\mathrm{r}-\mathrm{m})$. Second radiomedial (submarginal) cell rather long, not widened distally, 2.8-3.0 times as long as wide, 1.4-1.5 times as long as the narrow brachial (subdiscal) cell. First medial abscissa (1-SR+M) weakly sinuate. Second abscissa of medial vein (2-SR+M) short. Recurrent vein (m-cu) 5.6-6.5 times as long as second abscissa of medial vein $(2-\mathrm{SR}+\mathrm{M}), 0.6-0.7$ times as long as first radiomedial vein (2-SR). Mediocubital vein (M+CU1) weakly or distinctly curved in apical half. Distance from nervulus (cu-a) to basal (1-M) vein 0.6-0.8 times nervulus (cu-a) length. Brachial (subdiscal) cell closed weakly or rather distinctly behind recurrent vein $(\mathrm{m}-\mathrm{cu})$; posterior bulla present but short; posterior abscissa of anal vein $(2-1 \mathrm{~A})$ (behind brachial vein (CU1b)) present and short. Hind wing 5.2-5.5 times as long as wide. Recurrent vein $(\mathrm{m}-\mathrm{cu})$ almost straight, oblique towards base of wing, interstitial.

LeGs. Hind coxa 1.8-1.9 times as long as wide, $0.7-0.8$ times as long as propodeum. Hind femur 3.2 times as long as wide. Hind tarsus 0.9 times as long as hind tibia. Hind basitarsus without keel, with dense and long pale setosity. Second segment of hind tarsus 0.4 times as long as basitarsus, almost as long as fifth segment (without pretarsus).

Metasoma. Length 1.1-1.2 times as long as head and mesosoma combined, with sixth visible tergites, remaining segments almost not protruding behind sixth tergite. First tergite distinctly, curvedly and rather evenly widened from base to apex. Maximum width of tergite 2.2-2.3 times its minimum basal width; length 1.0-1.2 times its apical width, 1.0-1.2 times length of propodeum. Basal area of second tergite $0.25-0.30$ times as long as remaining part of tergite; apical area $0.8-0.9$ times as long as remaining part of tergite. Median length of second tergite (with areas) 0.7 times its basal width, 1.8-2.1 times median length of third tergite. Sixth tergite not enlarged, almost straight on posterior margin, without median emargination and postero-ventral lobes. Sixth tergite 0.5-0.9 times as long as fifth tergite; fifth tergite 0.8-1.1 times as long as fourth tergites. Ovipositor sheath $0.4-0.6$ times as long as metasoma, 0.7-0.9 times as long as mesosoma, $0.3-0.4$ times as long as fore wing.

SCUlPTURE AND PUBESCENCE. Vertex entirely and frons medially smooth, sometimes frons laterally weakly aciculate; face mainly smooth, only partly with very fine punctation and sometimes finely reticulate with striation laterally; temple smooth. Pro- and mesothorax mostly smooth, prothorax laterally with rugulose areas in large specimen; mesoscutum posteriorly with two short of long and straight carinae. Mesopleuron almost completely smooth. Propodeum mainly smooth, with large basolateral areas having fine reticulation along carinae; areola short and wide, entirely rugulose, almost as long as maximum width; median carina 1.5-2.0 times as long as anterior fork of areola. Hind legs mainly smooth, hind coxa dorsally partly rugulose. First tergite mainly striate-rugulose or reticulate-areolate with longitudinal striae, smooth on small or rather large medioapical area. Second tergite mainly smooth or partly finely coriaceous, coarsely striate on furrows and suture. Remaining tergites smooth, but fourth tergite distinctly crenulate on subbasal transverse furrow. Second and third tergites laterally distinctly and long striate-rugose. Vertex with very sparse, short and semi-erect pale setae posteriorly and laterally, glabrous on most part. Mesonotum mainly glabrous, with sparse short pale setae in medioposterior third. Mesopleuron mostly glabrous. Hind tibia dorsally with rather dense, long and short and semi-erect pale setae, its length $0.4-0.7$ times as long as maximum width of tibia. Laterotergites partly glabrous and partly in rather sparsely erect pale setae.

CoLour. Head black or dark reddish brown. Metasoma partly dark reddish brown to black, pronotum, propodeum, metapleuron and sometimes posterior part of mesopleuron reddish brown, yellowish brown to light reddish brown, propleuron sometimes reddish brown. Metasoma reddish brown, light reddish brown to partly yellowish brown, infuscate posteriorly. Antenna black or dark reddish brown, sometimes two basal segments brown with yellowish tint partly. Palps pale yellow. Legs almost entirely yellow 
with brownish tint. Ovipositor sheath light brown or brown in basal half and dark brown in apical half. Fore wing faintly, but distinctly and almost evenly infuscate. Pterostigma pale brown, paler basally and apically.

\section{Male}

MeASuREMENTs. Body length 2.9-3.3 mm; fore wing length 2.4-2.5 mm. Antenna 28-segmented. Legs. Hind femur wider, 2.6-2.7 times as long as wide.

Metasoma. Metasoma narrow. First metasomal tergite long and narrow, its length 1.4-1.6 times apical width; maximum width of tergite 1.6-2.0 times its minimum basal width. Basal area of second tergite weakly delineated posteriorly; apical area narrow, 0.5 times as long as remaining part of tergite. Median length of second tergite (with areas) almost equal to its basal width, 1.6-1.7 times median length of third tergite.

CoLOUR. Body lighter, but legs sometimes weakly infuscate. Otherwise similar to female.

\section{Diagnosis}

This new species differs from Ipodoryctes (A.) insignis Granger by having the areola of propodeum wide (vs narrow), brachial (subdiscal) cell of fore wing apically closed behind recurrent vein (m-cu) (vs before this vein), parallel vein (CU1a) of fore wing often arising from anterior $0.2-0.3$ of distal margin of brachial (subdiscal) cell (vs interstitial), hind femur entirely pale brown to yellow (vs dark reddish brown in posterior 0.6), first metasomal tergite wide, short and curved laterally (vs narrow, long and straight laterally), and second metasomal suture sinuate (vs evenly curved).

\section{Distribution}

Réunion.

Ipodoryctes (Afroipodoryctes) saintphilippensis sp. nov. urn:1sid:zoobank.org:act:D1CDBBD2-2E82-402B-88E5-4CFBAC361C70

Figs 20-21

\section{Etymology}

Named from Saint Philippe locality in Reunion Island, where all type material was collected.

\section{Material examined}

Holotype

REUNION • ; ; “La Reunion, ML 207350 m, Saint Philippe”, "Mare Longue, 13/19.IX.2001, Attie Marc leg", "forêt primaire, piege Malaise, au sol site 1"; MNHM.

\section{Paratypes}

REUNION • 1 q; "La Reunion, ML 19350 m, Saint Philippe", "Mare Longue, 20/26.II.2001, Attie Marc leg", "forêt primaire, piege Malaise, suspendu site 2"; MNHM • 1 đ̊?; "La Reunion, ML 33350 m, Saint Philippe", "Mare Longue, 10/16.III.2001, Attie Marc leg", "forêt primaire, piege Malaise, suspendu site 2"; MNHM • 1 ô; "La Reunion, ML 53350 m, Saint Philippe", "Mare Longue, 03/09.IV.2001, Attie Marc leg", "forêt primaire, piege Malaise, suspendu site 2"; MNHM・1 ô; "La Reunion, ML 90350 m, Saint Philippe", "Mare Longue, 16/22.V.2001, Attie Marc leg", "forêt primaire, piege Malaise, suspendu site 1"; MNHM • 1 क; "La Reunion, ML 157350 m, Saint Philippe", "Mare Longue, 2/8.VIII.2001, Attie Marc leg", "forêt primaire, piege Malaise, suspendu site 2"; IB-UNAM • 2 $ぇ$; " "La Reunion, ML 178350 m, Saint Philippe", "Mare Longue, 20/26.VIII.2001, Attie Marc leg", "forêt primaire, piege Malaise, suspendu site 1"; MNHM, IB-UNAM • 1 ㅇ, 1 ơ; "La Reunion, ML 214350 m, Saint 


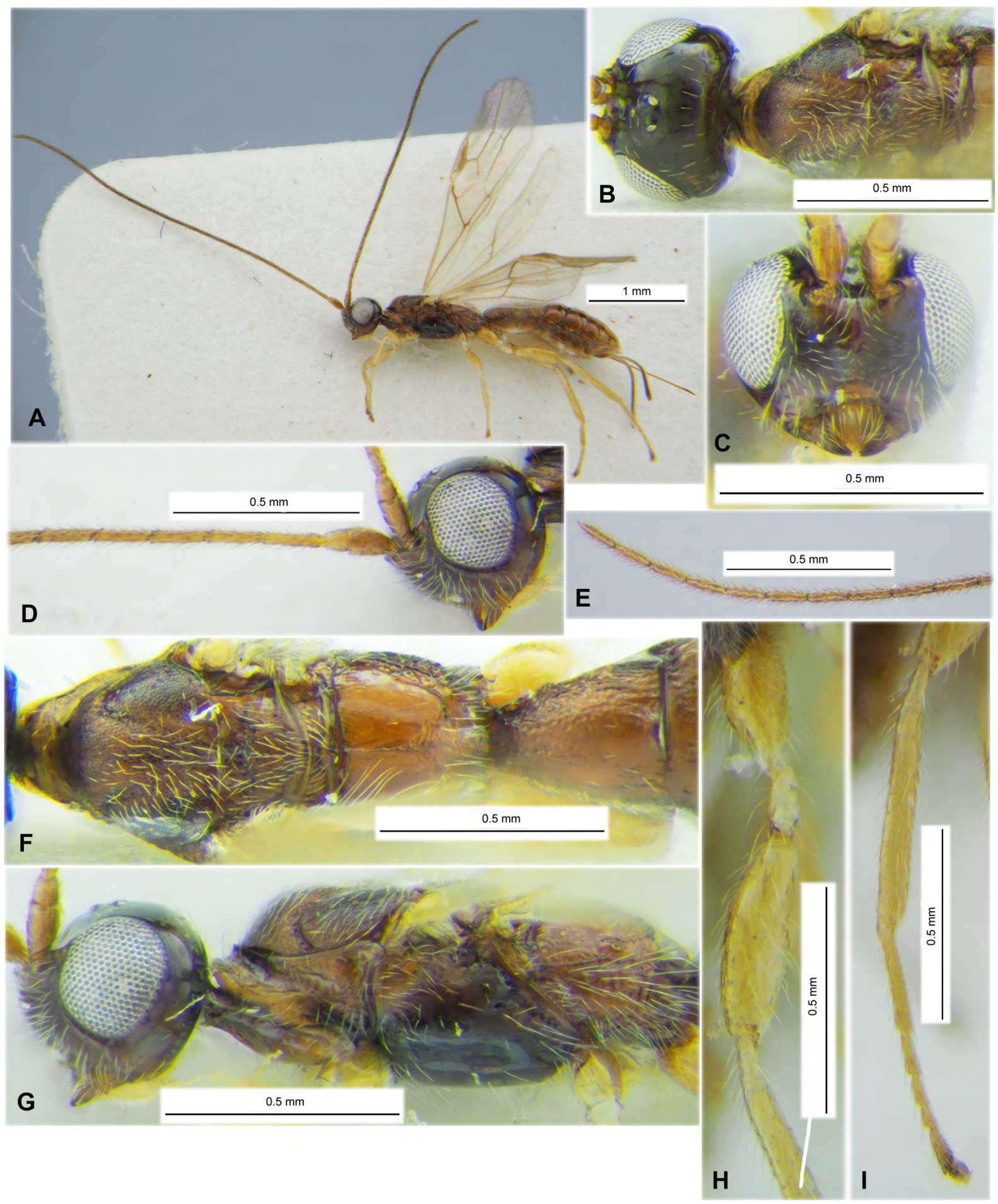

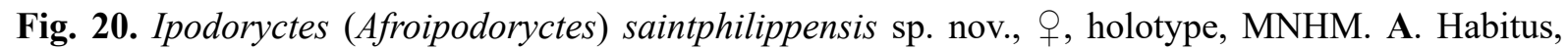
lateral view. B. Head and mesoscutum, dorsal view. C. Head, front view. D. Head and basal segments of antenna, lateral view. E. Apical segments of antenna. F. Mesosoma and first metasomal tergite, dorsal view. G. Head and mesosoma, lateral view. H. Hind coxa and femur. I. Hind tibia. 

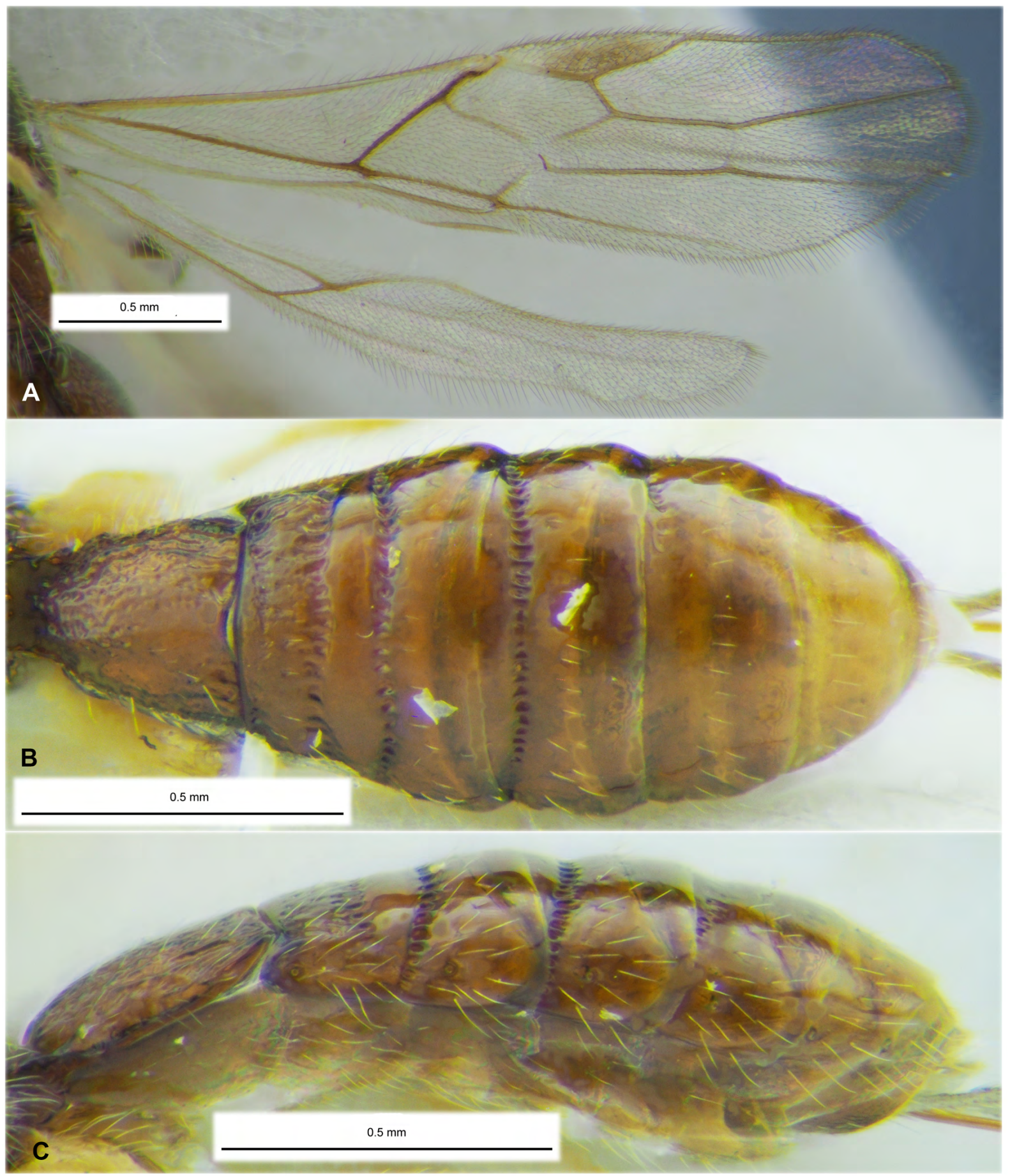

Fig. 21. Ipodoryctes (Afroipodoryctes) saintphilippensis sp. nov., + , holotype, MNHM. A. Wings. B. Metasoma, dorsal view. C. Metasoma, lateral view. 
Philippe", "Mare Longue, 19/25.IX.2001, Attie Marc leg", "forêt primaire, piege Malaise, suspendu site 2"; MNHM • 1 क; "La Reunion, ML 221350 m, Saint Philippe", "Mare Longue, 25.IX/01.X.2001, Attie Marc leg", "forêt primaire, piege Malaise, au sol site 2"; ZISP • 1 đơ; "La Reunion, ML 245320 m, Saint Philippe", "Mare Longue, 13/19.X.2001, Attie Marc leg", "plantation ONF, piege Malaise, au sol site 2"; MNHM• 1 ㅇ, 1 ग̃; "La Reunion, ML 248350 m, Saint Philippe", "Mare Longue, 19/25.X.2001, Attie Marc leg", "forêt primaire, piege Malaise, suspendu site 2"; MNHM • 1 \& [without metasoma]; "La Reunion, ML 255350 m, Saint Philippe", "Mare Longue, 25/31.X.2001, Attie Marc leg", "forêt primaire, piege Malaise, suspendu site 1", "RUS43 [= Jo929]"; MNHM • 2 ôें; "La Reunion, ML 297350 m, Saint Philippe", "Mare Longue, 30.XI/06.XII.2001, Attie Marc leg", "forêt primaire, piege Malaise, suspendu site 2"; MNHM • 1 ๆ "La Reunion, ML 266320 m, Saint Philippe", "Mare Longue, 31.X/06.XI.2001, Attie Marc leg", "plantation ONF, piege Malaise, suspendu site 2"; MNHM • 1 o "La Reunion, ML 287320 m, Saint Philippe", "Mare Longue, 18/24.XI.2001, Attie Marc leg", "plantation ONF, piege Malaise, au sol site 1"; MNHM • 1 "La Reunion, ML 308320 m, Saint Philippe", "Mare Longue, 06/12.XII.2001, Attie Marc leg", "plantation ONF, piege Malaise, au sol site 2"; MNHM • 1 ㅇ "La Reunion, ML 329320 m, Saint Philippe", "Mare Longue, 24/30.XII.2001, Attie Marc leg", "plantation ONF, piege Malaise, au sol site 2"; ZISP • 1 q "La Reunion, ML 312350 m, Saint Philippe", "Mare Longue, 12/18.XII.2001, Attie Marc leg", "forêt primaire, piege Malaise, au sol site 1"; MNHM • 1 §̊; "La Reunion, Saint-Denis, Colorado 650 m”, "vegetation secondaire, 22. iii.2003, Delvare G.", "forêt primaire, piege Malaise, suspendu site 1"; MNHM.

\section{Description}

\section{Female}

MEASUREMENTS. Body length 2.1-3.2 mm; fore wing length 1.9-2.7 mm.

HEAD. Head width 1.5-1.6 times its median length, about 1.1 times width of mesoscutum. Head behind eyes (dorsal view) distinctly and roundly narrowed. Transverse diameter of eye 1.8-2.2 times as long as temple. Ocelli small, arranged in almost equilateral triangle; POL 1.2-1.3 times OD, $0.4-0.5$ times OOL. Eye weakly emarginated opposite antennal socket, 1.2 times as high as broad. Malar space $0.25-0.30$ times height of eye, $0.7-0.8$ times basal width of mandible. Face width 0.9 times eye height and 1.4-1.5 times height of face and clypeus combined. Upper margin of clypeus situated weakly higher than lower level of eyes. Width of hypoclypeal depression $0.9-1.0$ times distance from depression to eye, 0.4 times width of face. Vertex convex. Head below eyes (front view) strongly and weakly-roundly narrowed. Antennae slender, almost filiform, 22-29-segmented, 1.2-1.3 times as long as body. Scapus 1.5-1.6 times as long as its maximum width, about 2.0 times length of pedicel. First flagellar segment 4.5-5.3 times as long as its apical width, about 1.1 times as long as second segment. Penultimate segment 4.75.3 times as long as its width, $0.7-0.8$ times as long as first segment, about 0.9 times as long as apical segment.

Mesosoma. Length 2.2-2.4 times its height. Pronotal carina high, situated submedially. Mesoscutum (dorsal view) 0.9 times as long as wide. Notauli distinct and rather deep in anterior half, fine and shallow in posterior half, rather narrow, densely crenulate with granulation partly. Median lobe of mesoscutum distinctly convex anteriorly. Prescutellar depression shallow, with 3-5 carinae, finely granulatereticulate, weakly curved along posterior margin, 0.4 times as long as scutellum. Scutellum almost as long as its maximum width. Subalar depression weakly and shortly crenulate. Sternaulus (precoxal furrow) entirely smooth, with subround distinct pit posteriorly. Prepectal carina below without low lobes opposite fore coxae. Propodeum weakly arcuately sloping backward (lateral view). Metapleural flange posteriorly with very sparse long setae.

WINGS. Length of fore wing 3.6-3.8 times its maximum width. Pterostigma 3.8-4.0 times as long as wide. Metacarpus (1-R1) 1.3-1.4 times as long as pterostigma. Radial (marginal) cell 3.6-3.8 times 
as long as wide. Radial vein (r) arising weakly before middle of pterostigma. First radial abscissa (r) forming obtuse corner with second abscissa (3-SR). Second radial abscissa (3-SR) 3.3-3.8 times as long as first abscissa (r), 0.6 times as long as the straight third abscissa (SR1), 1.4-1.5 times as long as second radiomedial vein ( $\mathrm{r}-\mathrm{m})$. Second radiomedial (submarginal) cell long, not widened distally, 3.2-3.3 times as long as wide, 1.4-1.5 times as long as the narrow brachial (subdiscal) cell. First medial abscissa (1$\mathrm{SR}+\mathrm{M})$ weakly sinuate. Second abscissa of medial vein $(2-\mathrm{SR}+\mathrm{M})$ short. Recurrent vein $(\mathrm{m}-\mathrm{cu}) 3.5-4.0$ times as long as second abscissa of medial vein $(2-\mathrm{SR}+\mathrm{M}), 0.6$ times as long as first radiomedial vein (2-SR). Mediocubital vein (M+CU1) almost straight. Distance from nervulus (cu-a) to basal (1-M) vein almost equal to nervulus (cu-a) length. Parallel vein (CU1a) not interstitial, arising from anterior $0.2-0.3$ of vein closed brachial (subdiscal) cell distally. Brachial (subdiscal) cell closed weakly behind recurrent vein (m-cu); posterior bulla present but short; posterior abscissa of anal vein (2-1A) (behind brachial vein (CU1b)) very short. Hind wing 4.8-5.6 times as long as wide. Recurrent vein (m-cu) curved, oblique towards base of wing, antefurcal.

Legs. Hind coxa about 2.0 times as long as wide, $0.8-0.9$ times as long as propodeum. Hind femur 2.8-3.0 times as long as wide. Hind tarsus $0.90-0.95$ times as long as hind tibia. Hind basitarsus without keel, with dense and distinct pale setosity. Second segment of hind tarsus 0.4 times as long as basitarsus, as long as fifth segment (without pretarsus).

Metasoma. Length 1.1 times as long as head and mesosoma combined, with sixth visible tergites, remaining segments weakly protruding behind sixth tergite. First tergite distinctly, evenly and weakly convexly widened from base to apex. Maximum width of tergite 2.2-2.4 times its minimum basal width; length 1.15-1.20 times its apical width, 1.1 times length of propodeum. Basal area of second tergite weakly delineated by shallow furrow, $0.30-0.35$ times as long as remaining part of tergite; apical area 0.6-0.7 times as long as remaining part of tergite. Median length of second tergite (with areas) 0.6 times its basal width, 1.4-1.5 times median length of third tergite. Second suture distinctly sinuate. Sixth tergite not enlarged, almost straight in posterior margin, without median emargination and posteroventral lobes. Sixth tergite $0.7-0.8$ times as long as fifth tergite; fifth tergite $1.1-1.3$ times as long as fourth tergites. Ovipositor sheath $0.5-0.6$ times as long as metasoma, $0.70-0.85$ times as long as mesosoma, $0.30-0.35$ times as long as fore wing.

SCULPTURE AND PUBESCENCE. Head mostly smooth, only face partly punctate. Prothorax mostly smooth, its sides partly finely rugose. Mesoscutum and scutellum entirely densely granulate; mesoscutum rugose but without striae in its medioposterior third. Mesopleuron almost entirely smooth. Metapleuron very finely rugulose in anterior half, distinctly rugose-reticulate in posterior half. Propodeum mainly smooth, with large smooth or partly finely reticulate basolateral areas; areola short and wide, finely rugulose, 1.0-1.2 times as long as its maximum width; median carina 1.2-1.3 times as long as anterior fork of areola. Hind legs mainly smooth. First tergite mainly rugulose-reticulate and partly with sparse longitudinal and weakly curved striae, smooth on small medio-apical area. Second tergite interruptedly rugose-striate with smooth basal and apical areas, distinctly crenulate on furrows and suture. Remaining tergites smooth, but fourth tergite distinctly crenulate on subbasal transverse furrow. Third-sixth tergites laterally entirely smooth. Vertex mainly glabrous, with sparse, short and semi-erect pale setae posteriorly and laterally. Mesonotum almost completely in short semi-erect yellow setae. Mesopleuron mostly glabrous. Hind tibia dorsally with rather dense, long and short semi-erect white setae, its length about $0.4-0.6$ times as long as maximum width of tibia.

CoLOUR. Head dark reddish brown to black. Metasoma mostly reddish brown with darks sports ventrally and on mesopleuron. Metasoma reddish brown to light reddish brown. Antenna dark reddish brown, 6-7 basal segments reddish brown. Palps yellow. Legs yellow with brownish tint. Ovipositor sheath dark 
brown to black. Fore wing distinctly and almost evenly infuscate. Pterostigma mainly brown and partly pale or yellow and partly brownish.

\section{Male}

MEASUREMENTs. Body length 1.7-3.1 mm; fore wing length $1.5-2.3 \mathrm{~mm}$.

HEAD. Antenna 19-26-segmented.

LEGS. Hind femur wide, 2.6-2.8 times as long as maximum width.

Metasoma. Metasoma slender, 1.1-1.2 times as long as head and mesosoma combined. First tergite long and narrow, its length 1.4-1.6 times maximum width of tergite, which is 1.8-2.0 times its minimum basal width. Basal area of second tergite 0.2-0.4 times as long as remaining part of tergite; apical area short, $0.3-0.5$ times as long as remaining part of tergite; areas sometimes indistinctly delineated. Median length of second tergite (with areas) 1.0-1.1 times its basal width, 1.5-1.8 times median length of third tergite. Third-fifth tergites rugose-striate in basal $0.5-0.3$.

Colour. Rarely body paler. Pterostigma sometimes mostly brown, pale basally and apically. Metasoma sometimes light brown or light reddish brown. Otherwise similar to female.

\section{Diagnosis}

This new species distinctly differs from the remaining two described species of the subgenus Afroipodoryctes [I. (A.) insignis Granger and I. (A.) reunionus sp. nov.] by having the mesoscutum entirely densely and distinctly granulate and almost entirely covered by short pale setae (vs smooth and mainly glabrous).

\section{Distribution}

Réunion.

Genus Leptorhaconotus Granger, 1949

Leptorhaconotus Granger, 1949: 137.

Leptorhaconotus - Shenefelt \& Marsh 1976: 1316. — Quicke 1996: 111. — Yu et al. 2016. — JassoMartínez et al. 2019: 168.

\section{Type species}

Leptorhaconotus brunneus Granger, 1949, by original designation.

Description (Figs 22-23)

HEAD. Weakly depressed dorso-ventrally (lateral view), not high, weakly transverse. Ocelli arranged in almost equilateral triangle. Frons not concave, flat, with shallow median furrow. Eyes with sparse and very short setae. Occipital carina dorsally complete, joined ventrally with hypostomal carina. Malar suture absent. Clypeal suture present, complete, entirely distinct. Hypoclypeal depression medium size, subround. Postgenal bridge present, but narrow. Palps medium length; labial palps short, 4-segmented, its third segment not shortened. Scapus wide and rather long, without apical lobe and basal constriction, its ventral margin (lateral view) not longer than dorsal margin. First flagellar segment subcylindrical, almost straight, weakly shorter than second segment. 
Mesosoma. Weakly depressed, long. Neck of prothorax rather elongate. Pronotum dorsally rather distinctly convex, with pronotal carina distinct, high and widely separate from anterior margin of mesoscutum; pronope absent. Propleural dorsoposterior lobe short and wide. Mesonotum weakly gently elevated above pronotum, entirely densely granulate. Median lobe of mesonotum without median longitudinal furrow and without anterolateral corners. Notauli distinct and complete, but shallow. Tegula short, widened distally, almost straight along its posterior margin. Prescutellar depression rather long, with distinct median carina. Lateral longitudinal wing-like flanges on the level of prescutellar depression long and low. Scutellum weakly convex, without lateral carinae. Subalar depression shallow and rather narrow. Sternaulus (precoxal sulcus) absent or very shallow and almost indistinct. Mesopleural pit deep, subround, connected directly with mesopleural suture. Metanotum with short, wide and rounded median tooth (lateral view). Prepectal carina distinct and complete, not high below. Metapleural flange short, rather wide, rounded apically. Propodeum without delineated areas; lateral tubercles and propodeal bridge absent. Propodeal spiracles small and round.

Wings. Pterostigma of fore wing narrow and long. Radial vein (r) arising from middle of pterostigma. Radial (marginal) cell not shortened. Both radiomedial veins (2-SR, r-m) present. Second radiomedial (submarginal) cell long and rather narrow. Recurrent vein (m-cu) weakly postfurcal. Discoidal (discal) cell petiolate anteriorly, petiole (1-SR) rather long. Nervulus (cu-a) distinctly postfurcal. Brachial (subdiscal) cell evenly and curvedly closed postero-apically weakly before recurrent vein (m-cu), without antero-posterior corner, but with short additional abscissa. Parallel vein (CU1a) interstitial. Transverse anal veins $(2 \mathrm{~A}$, a) absent. Hind wing with three hamuli. Radial (marginal) cell weakly narrowed apically, without transverse vein (r). Medial (basal) cell distinctly widened towards apex. Nervellus (cua) present. Submedial (subbasal) cell rather short. First abscissa of mediocubital vein $(\mathrm{M}+\mathrm{CU})$ shorter than second abscissa (1-M). Recurrent vein (m-cu) rather long, strongly oblique towards base of wing, unsclerotised, but pigmented.

LEGS. Fore tibia with several short and slender pale spines arranged in single row. Middle tibia without spines. Middle tarsal segments rather long. Hind coxa long and wide, without basoventral corner and tubercle. Fore and middle femora with low and wide dorsal protuberances. Hind femur wide, without dorsal protuberance. Inner spur of hind tibia simple, 0.4 times as long as hind basitarsus. Hind basitarsus about 0.7 times as long as second-fifth segments combined. Claws thick, short and simple.

Metasoma. Slender in female, rather narrow, weakly widened from base to third segment, then almost parallel-sided, with seven visible dorsally tergites, tergites fourth to sixth more or less distinctly narrowed basally, laterotergites (epipleura) separated on second-fifth tergites. Metasomal tergites in apical half distinctly and at long distance bend down; ventral margins of sixth tergite almost connected below or closely situated. First segment semi-petiolate, long and rather wide, laterally distinctly bend down. Acrosternite of first segment elongated, about 0.35 times as long as first tergite, its apical margin situated behind level of spiracles. Dorsope of first tergite shallow and small; basolateral processes and spiracular tubercles absent, spiracles situated in basal 0.3 of tergite; dorsal carinae indistinct. First and second tergites not fused immovably. Second tergite with wide and long subtriangular basal area separated by not deep lateral convergent furrows; without any transverse subapical furrow and area. Second suture shallow or very shallow, complete and almost straight. Third tergite without transverse depression or furrow. Fifth tergite about as long as fourth tergite, but about twice longer than sixth tergite. Sixth and seventh tergites distinctly protruding behind fifth, coarsely sclerotised, not enlarged, smooth in apical halves, about half as long as fifth tergite. Hypopygium along apical margin with distinct and rather deep emargination. Ovipositor thick, curved up, usually wide and flattened in basal 0.5-0.7, slender and pointed in apical part, but sometimes subround in dissection (L. alluaudi Granger, 1949 and L. annulicornis Granger, 1949). Ovipositor sheath leaf-shaped and different sizes, wide, weakly 
concave, short. Male metasoma without female transformation and very narrow, its tergites more slender and more distinctly sculptured.

\section{Diagnosis}

This genus morphologically distinctly differs from the remaining genera of Rhaconotini (see key to genera upper) by the following combination of morphological features: 1) hind coxa without basoventral corner and tubercle; 2) sternaulus (precoxal sulcus) absent or almost absent; 3) propodeum without any delineated areas; 4) metasomal tergites in apical half distinctly and usually at long distance bend down and ventral margins of sixth tergite below almost connected or closely situated; 6) first metasoma segment semi-petiolate, its acrosternite elongate and about 0.35 times as long as first tergite, first tergite laterally distinctly bend down; 7) with seven dorsally visible tergites; 8) hypopygium along apical margin with distinct and rather deep emargination; 9) ovipositor sheath leaf-shaped, wide, weakly concave and considerably short.

\section{Composition}

Leptorhaconotus alluaudi Granger, 1949 (AF); L. annulicornis Granger, 1949 (AF); L. brunneus Granger, 1949 (AF); L. coriaceus Granger, 1949 (AF); L. marshi Quicke, 1996 (AF); L. seyrigi Granger, 1949 (AF); L. testaceus Granger, 1949 (AF).

\section{Hosts}

Unknown.

\section{Distribution}

Afrotropical region.

\section{Remarks}

This genus was previously placed within the monotypic tribe Leptorhaconotini (van Achterberg 1984; Belokobylskij 1992). Recent molecular phylogenetic studies (Zaldívar-Riverón et al. 2008; JassoMartínez et al. 2019) however, consistently showed that it actually belongs to the Rhaconotini. Species of Leptorhaconotus are characterized by having various exclusive morphological features, including metasomal tergites laterally evenly bend down at long distances; ventral margins of sixth tergite below almost connected or closely situated; metasoma tube-shaped in dissection; acrosternite of first metasomal segment elongated; ovipositor basally flat and wide, evenly narrowed toward apex, strong upcurved, without dorsoapical tubercles and ventro-apical serration; and ovipositor sheath wide and flat, leaf-shaped. All listed characters convinced us to preserve this supergeneric name as subtribe name Leptorhaconotina inside Rhaconotini

\section{Leptorhaconotus brunneus Granger, 1949}

Figs 22-23

Leptorhaconotus brunneus Granger, 1949: 138.

Leptorhaconotus brunneus - Shenefelt \& Marsh 1976: 1316. — Yu et al. 2016. — Jasso-Martínez et al. 2019: 167.

\section{Material examined}

Holotype

MADAGASCAR: • + ; "Madagascar, Bekily, reg. sud de l'ile", "Museum Paris, II. 37, A. Seyrig", "42", "Type", "q Leptorhaconotus brunneus Granger, C. van Achterberg, 1980, Holotype"; MNHN. 


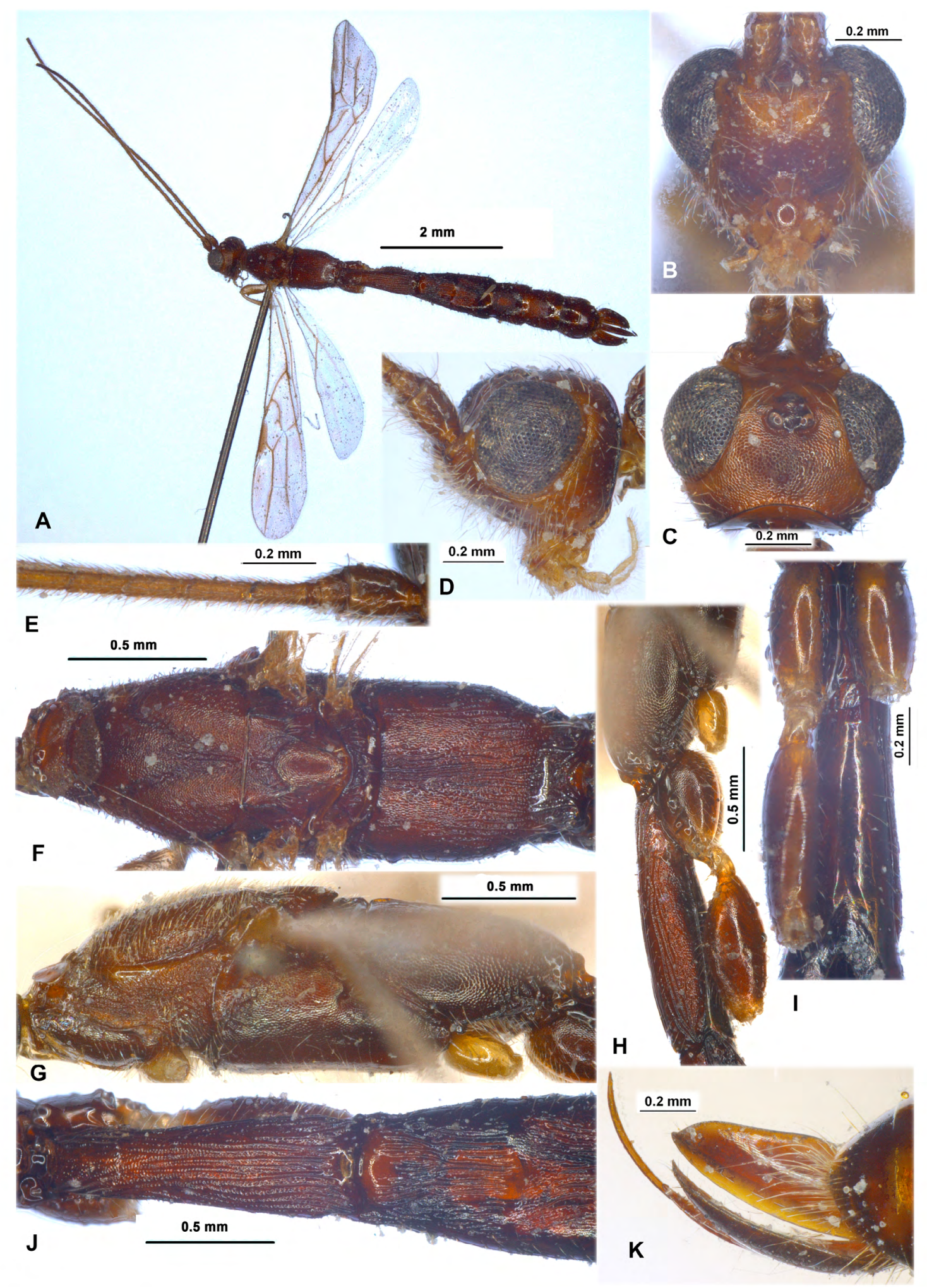

Fig. 22. Leptorhaconotus brunneus Granger, 1949,, , holotype, MNHN. A. Habitus, dorsal view. B. Head, front view. C. Head, dorsal view. D. Head, lateral view. E. Basal segments of antenna. F. Mesosoma, dorsal view. G. Mesosoma, lateral view. H. Propodeum, first metasomal tergite, hind coxa and femur, lateral view. I. Hind coxae and first metasomal tergite, ventral view. J. First to third metasomal tergites, dorsal view. K. Ovipositor with sheaths, dorso-lateral view. 

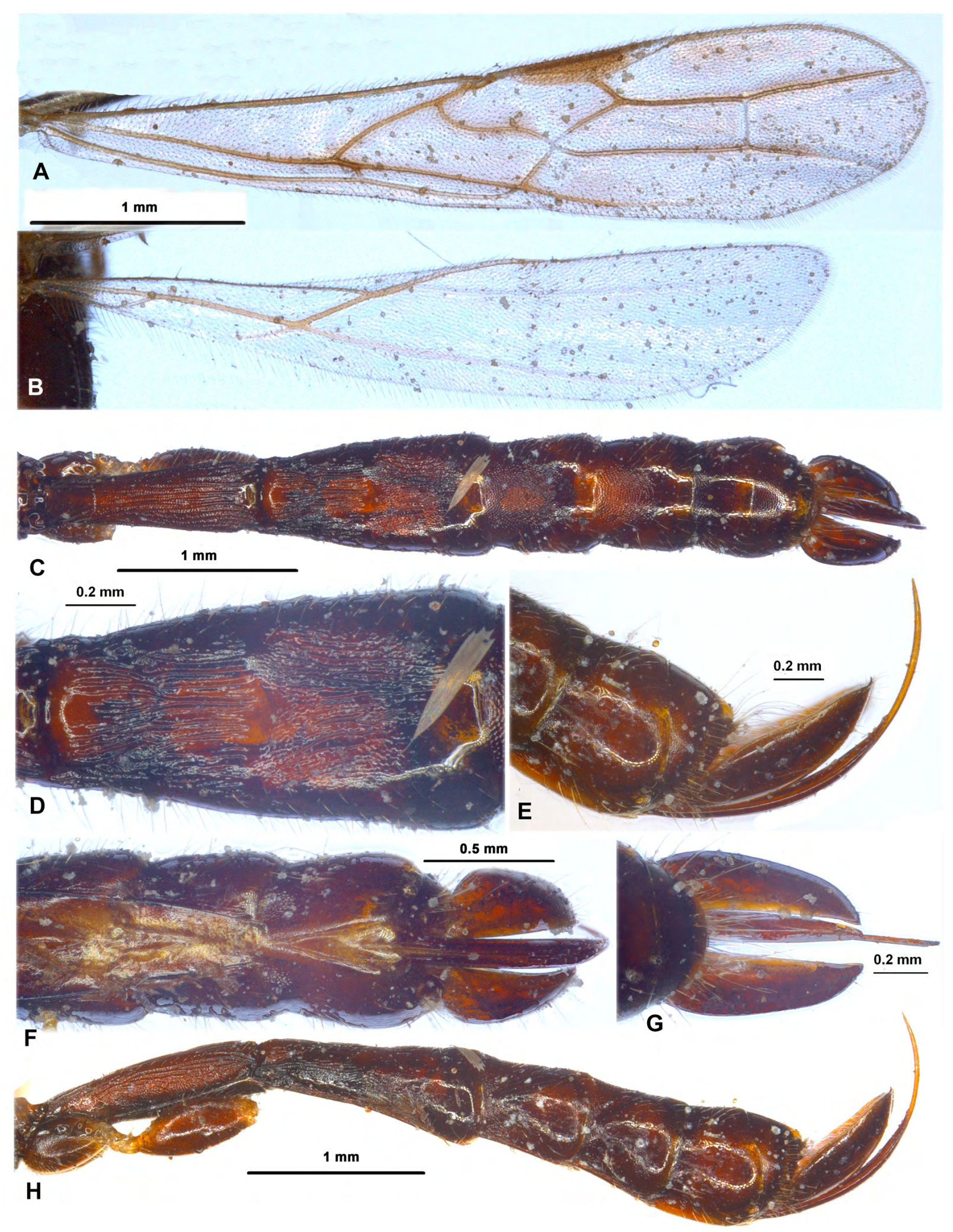

Fig. 23. Leptorhaconotus brunneus Granger, 1949, + , holotype, MNHN. A. Fore wing. B. Hind wing. C. Metasoma, dorsal view. D. Second and third metasomal tergites, dorsal view. E. Apex of metasoma and ovipositor with sheaths, lateral view. F. Apex of metasoma and ovipositor with sheaths, ventral view. G. Ovipositor with sheaths, dorsal view. H. Metasoma, lateral view. 


\section{Description}

Female

Measurements. Body length $7.3 \mathrm{~mm}$; fore wing length $4.3 \mathrm{~mm}$.

HEAD. Width 1.35 times its median length, 1.2 times width of mesoscutum. Head behind eyes (dorsal view) distinctly and almost linearly narrowed. Transverse diameter of eye 2.6 times length of temple. Ocelli medium-sized, arranged in almost equilateral triangle; POL 0.8 times OD, 0.5 times OOL. Eye weakly emarginated opposite antennal sockets, 1.1 times as high as broad. Malar space height 0.4 times height of eye, almost equal to basal width of mandible. Face width equal to height of eye and 1.25 times height of face and clypeus combined. Clypeus flat (lateral view). Upper margin of clypeus situated weakly upper lower level of eyes. Hypoclypeal depression round, its width 0.8 times distance from edge of depression to eye, 0.4 times width of face. Vertex almost flat. Head below eyes strongly and almost linearly narrowed Antennae slender, weakly setiform, more than 33-segmented (apical segments missing). Scapus 1.6 times as long as its maximum width. First flagellar segment 2.8 times as long as its apical width, as long as second segment. Subapical segments 2.2 times as long as their width.

Mesosoma. Length 3.0 times its height. Mesoscutum (dorsal view) 1.1 times as long as maximum width. Notauli distinct, shallow (especially posteriorly), complete, rather narrow, densely rugose-crenulate. Median lobe of mesoscutum convex anteriorly. Prescutellar depression long, widened postero-laterally, with distinct median and several lateral carinae, distinctly rugose-reticulate between carinae, medially 0.35 times as long as scutellum. Scutellum about 1.5 times as long as its anterior maximum width. Subalar depression shallow, rather densely and distinctly rugose-granulate. Sternaulus (precoxal sulcus) absent. Prepectal carina below without wide lobes opposite fore coxae. Metanotum dorso-medially with three fine carinae (dorsal view). Metapleural flange without dense pubescence.

WINGs. Fore wing 4.5 times its maximum width. Metacarpus (1-R1) 1.35 times as long as pterostigma. Radial (marginal) cell 4.7 times as long as wide. Radial vein (r) arising almost from middle of pterostigma. First radial abscissa (r) forming very obtuse angle with second abscissa (3-SR). Second radial abscissa (3-SR) almost 4.0 times as long as first abscissa (r), 0.75 times as long as the straight third abscissa (SR1), 1.6 times as long as first radiomedial vein (2-SR). Second radiomedial (submarginal) cell not widened distally, almost 4.0 times as long as wide, 1.25 times as long as the narrow brachial (subdiscal) cell. First medial abscissa $(1-\mathrm{SR}+\mathrm{M})$ strongly sinuate. Recurrent vein $(\mathrm{m}-\mathrm{cu})$ very weakly postfurcal, 0.6 times as long as first radiomedial vein (2-SR). Mediocubital vein (M+CU1) weakly curved to anal vein (21A) in distal half. Nervulus (cu-a) strongly postfurcal, distance between basal (1-M) vein and nervulus (cu-a) 1.4 times nervulus (cu-a) length. Parallel vein (CU1a) interstitial; posterior bulla of brachial vein (CU1b) short, posterior abscissa of anal vein (2-1A) (behind brachial vein (CU1b)) present, but very fine; brachial vein (CU1b) distinctly oblique towards base of wing. Hind wing 5.4 times as long as wide. Recurrent vein $(\mathrm{m}-\mathrm{cu})$ almost straight, strongly oblique towards base of wing, very faintly pigmented.

LeGS. Hind coxa 1.6 times as long as wide (with basoventral tubercle). Hind femur wide, its length 2.3 times maximum width.

Metasoma. Length 1.6 times as long as head and mesosoma combined, with seven coarsely sclerotised and at least basally (posterior tergites) sculptured visible tergites. First tergite weakly and almost linearly widened from base to subapex, weakly narrowed apically. Maximum subapical width of first tergite 1.5 times its minimum basal width; length 3.2 times apical width, 1.7 times length of propodeum. Second tergite without apical area, with long and wide subtriangular basal area, median length this area almost equal to length of remaining tergite. Median length of second tergite 1.6 times its basal width, almost equal to length of third tergite. Second suture very shallow, almost indistinct, with wide straight smooth transverse stripe on suture place. Sixth tergite not enlarged, distinctly concave on posterior 
margin, without median emargination and posteroventral lobes. Sixth tergite 0.7 times as long as fifth tergite, about as long as seventh tergite. Ovipositor sheath flat, leaf-like, very wide, evenly narrowed towards apex and towards base, about 3.0 times as long as its maximum width, 0.8 times as long as first metasomal tergite. Ovipositor thick, curved up, wide and flattened dorso-ventrally in basal 0.7 , slender and pointed in apical 0.3 .

SCUlPTURE AND PUBESCENCE. Vertex and frons densely and distinctly granulate, becoming finer on frons; face very finely punctate and coriaceous between punctation; temple densely granulate becoming granulate-coriaceous below. Mesoscutum entirely densely granulate, without rugosity along notauli, narrow rugulose-striate in its medioposterior half. Scutellum densely and finely granulate. Mesopleuron densely and finely granulate becoming finer below. Metapleuron densely granulate, with rugosity posteriorly. Propodeum without areolation, with distinct and dense longitudinal striation and dense fine granulation between striae, mainly granulate and with fine and incomplete striae in basolateral halves. Hind coxa dorsally finely granulate-coriaceous, smooth in lower half. Hind femur mainly smooth. First entirely, second mainly (except smooth basomedian place) and third in basal $0.7-0.8$ tergites densely and coarsely striate with numerous fine rugulosity between striae; third tergite rugulose-granulate on subposterior 0.2 becoming smooth posteriorly. Fourth and fifth tergites entirely densely and rather coarsely rugulose-areolate with granulation in basal 0.5-0.6 and smooth apically. Sixth and seventh tergites finely or very finely granulate-coriaceous in basal half and smooth apically. Third-seventh tergites laterally densely and finely reticulate-granulate or reticulate-coriaceous in basal $0.5-0.3$ and smooth on remaining parts. Vertex widely with rather dense, short and semi-erect pale setae, glabrous on rather small medio-anterior area. Mesoscutum entirely in dense, short and semi-erect golden setae. Mesopleuron glabrous on wide median area, in sparse and erect pale setae on rest parts.

Colour. Head brownish yellow, darker dorsally, behind eyes and in lower 0.7 of face. Mesosoma reddish brown in upper half and dark brown to black in lower half. Metasoma reddish brown, dark reddish brown to black medially. Antennae brownish yellow, darkened towards apex, scapus mainly dark brown. Palps yellow. Fore leg entirely and middle coxa yellow (other parts of middle leg missing). Hind coxa and femur dark brown, but yellowish apically (other parts of this leg missing). Ovipositor sheath dark brown, but paler marginally. Fore wing faintly maculate usually along veins. Pterostigma brown, yellow basally and in apical third.

\section{Male}

Unknown.

\section{Distribution}

Madagascar.

Genus Mimipodoryctes Belokobylskij, 2000

Mimipodoryctes Belokobylskij, 2000: 348.

Mimipodoryctes - Belokobylskij \& Maeto 2006: 704; 2009: 305. — Yu et al. 2016. — Jasso-Martínez et al. 2019: 165.

\section{Type species}

Mimipodoryctes robustus Belokobylskij, 2000 (= Rhyssalus rubriceps Cameron, 1909). 


\section{Description (Figs 24-25)}

HEAD. Head not depressed, transverse. Ocelli arranged in weakly obtuse triangle. Frons not concave, without median keel or furrow. Eyes glabrous. Occipital carina complete dorsally, joined below with hypostomal carina above base of mandible. Malar suture indistinct. Clypeal suture complete, deep laterally and shallow upper. Hypoclypeal depression medium-sized and subround. Postgenal bridge narrow. Palps long; maxillary palps 6-segmented, its sixth segment about as long as fifth segment; labial palps 4-segmented, its third segment not shortened. Scapus of antenna rather wide and long, without apical lobe and basal constriction; its ventral margin (lateral view) not longer than dorsal margin. First flagellar segment subcylindrical, almost straight, longer than second segment. Apical segment acuminate apically, without spine.

Mesosoma. Mesosoma not depressed and rather long. Neck of prothorax rather short. Pronotum dorsally almost flat, with distinct submedian pronotal carina; pronope absent. Propleural dorsoposterior lobe rather long and wide. Mesonotum highly, gently and roundly elevated above pronotum; entirely distinctly rugose-granulate and entirely cover by dense setae. Median lobe of mesonotum without median longitudinal furrow and anterolateral corners. Notauli entirely rather deep, complete, densely and distinctly crenulate. Tegula widened distally, weakly convex along its posterior margin. Prescutellar depression long, rather deep, with several carinae. Lateral longitudinal wing-like flanges on level of prescutellar depression rather high. Scuto-scutellar suture distinct and complete. Scutellum weakly convex, with distinct lateral carinae at least in basal half. Metanotum with short, but distinct and subpointed median tooth or sometimes without it. Mesopleural pit deep, round, connected with mesopleural suture. Sternaulus (precoxal sulcus) rather deep, narrow, long, weakly curved, almost smooth. Prepectal carina distinct and complete, weakly widened below opposite fore coxae. Postpectal carina absent. Metapleural flange long, narrow and rounded apically, covered by very dense white setae. Propodeum with basolateral areas distinctly delineated by carinae, without areola; lateral tubercles small; propodeal bridge absent. Propodeal spiracles small and round.

Wings. Pterostigma of fore wing wide. Radial vein (r) arising almost from middle of pterostigma. Radial (marginal) cell not shortened. Both radiomedial veins (2-SR, r-m) present. Second radiomedial (submarginal) cell rather short and wide. Recurrent vein (m-cu) postfurcal or sometimes almost interstitial. Discoidal (discal) cell petiolate anteriorly, petiole (1-SR) rather long; basal (1-M) and recurrent (m-cu) veins subparallel. Nervulus (cu-a) postfurcal. Brachial (subdiscal) cell rather strongly, obliquely and linearly closed apically distinctly behind recurrent vein (m-cu). Parallel vein (CU1a) not interstitial, arising almost from or slightly before or behind middle of brachial vein (CU1b). Transverse anal veins $(2 \mathrm{~A}, \mathrm{a})$ absent. Hind wing with three hamuli. Radial vein (SR) arising from costal vein $(2-\mathrm{SC}+\mathrm{R})$ distinctly far from basal vein $(1 \mathrm{r}-\mathrm{m})$. Radial (marginal) cell subparallel in basal half and very weakly roundly narrowed toward apex, without additional transverse vein (r). Medial (basal) cell distinctly widened towards apex, $0.45-0.50$ times as long as hind wing. Nervellus (cu-a) present. Submedial (subbasal) cell medium sized. First abscissa of mediocubital vein (M+CU) 0.6-0.9 times as long as second abscissa (1-M). Recurrent vein (m-cu) rather short, antefurcal, strongly oblique towards base of wing, weakly sclerotised.

LEGS. Fore and middle tibiae with rather dense short and thick spines arranged in narrow stripe. Middle tarsal segments rather short. Hind coxa rather short and wide, with distinct basoventral corner and tubercle. All femora with more or less distinct dorsal protuberances. Hind femur wide. Inner spur of hind tibia almost entirely shortly setose, about 0.3 times as long as hind basitarsus. Hind basitarsus $0.7-0.9$ times as long as second-fifth segments combined. Claws short, thick and simple.

Metasoma. Metasoma with six (female) or seven (male) visible, coarsely sclerotised and sculptured tergites. First metasomal tergite not petiolate, wide, immovably fused with second tergite in female, 
not fused in male (see laterally). Acrosternite of first segment about 0.2 times as long as first tergite, its apical margin placed distinctly before spiracles. Dorsope of first tergite small but distinct; usually present short and rather wide basolateral lobes; spiracular tubercles indistinct, spiracles situated in basal third of tergite; dorsal carinae usually complete. Second suture distinct and deep. Second tergite with short or sometimes very short (almost indistinct) semi-oval basal area, with narrow apical area delineated anteriorly by fine furrow and different type of sculpture. Third tergite without transverse depression or furrow. Second-sixth tergites with separate laterotergites. Sixth tergite of female rather large, often with median emargination on posterior margin, longer than previous tergite, covered the following segments. Hypopygium small, obtuse medioposteriorly. Ovipositor shorter or longer than metasoma. Apical part of ovipositor with single or two small dorsal nodes and serrate ventrally.

\section{Diagnosis}

Mimipodoryctes Belokobylskij, 2000 is morphologically similar to Ipodoryctes Granger, 1949 but differs from the latter genus by having immovably fused first and second metasomal tergites in females. This genus differs from Arhaconotus Belokobylskij, 2000, also having immovably fused first and second metasomal tergites, by parallel vein (CU1a) of fore wing not interstitial (vs interstitial), first and second tergites of male not fused immovably (vs fused in male), mesopleuron entirely densely pubescent (vs not entirely and sparsely setose), first abscissa of mediocubital vein ( $\mathrm{M}+\mathrm{CU})$ of hind wing long (vs short), laterotergites entirely coved by long and dense setae (vs almost glabrous) and vertex and mesoscutum striate or rugose-granulate (vs usually granulate).

\section{Composition}

Mimipodoryctes korotyaevi (Belokobylskij, 1996) (OR); M. peregrinus (Belokobylskij, 1994) (OR); M. rokkoensis Belokobylskij \& Maeto, 2006 (PA); M. rubriceps (Cameron, 1909) (OR, AU).

\section{Hosts}

Unknown.

\section{Distribution}

Australasian, Oriental and Palaearctic regions.

\section{Remarks}

Only one species of Mimipodoryctes was included in molecular phylogenetic study by Jasso-Martínez et al. (2019), whereas no specimens of Arhaconotus were examined. However, some morphological characters of these two genera suggest their close relationship.

A key to all described species Mimipodoryctes was published by Belokobylskij \& Maeto (2009: 306).

Mimipodoryctes rubriceps (Cameron, 1909)

Figs 24-25

Rhyssalus rubriceps Cameron, 1909: 114 (BMNH; examined).

Mimipodoryctes robustus Belokobylskij, 2000: 348 (ZISP; examined).

Ipodoryctes rubriceps - Belokobylskij 1996a: 161; 2001: 156.

Mimipodoryctes rubriceps - Belokobylskij \& Maeto 2006: 704; 2009: 306. — Yu et al. 2016. — JassoMartínez et al. 2019: 167.

Mimipodoryctes robustus - Belokobylskij \& Maeto 2006: 704 (as synonym of M. rubriceps); 2009: 305 (as synonym of M. rubriceps). 

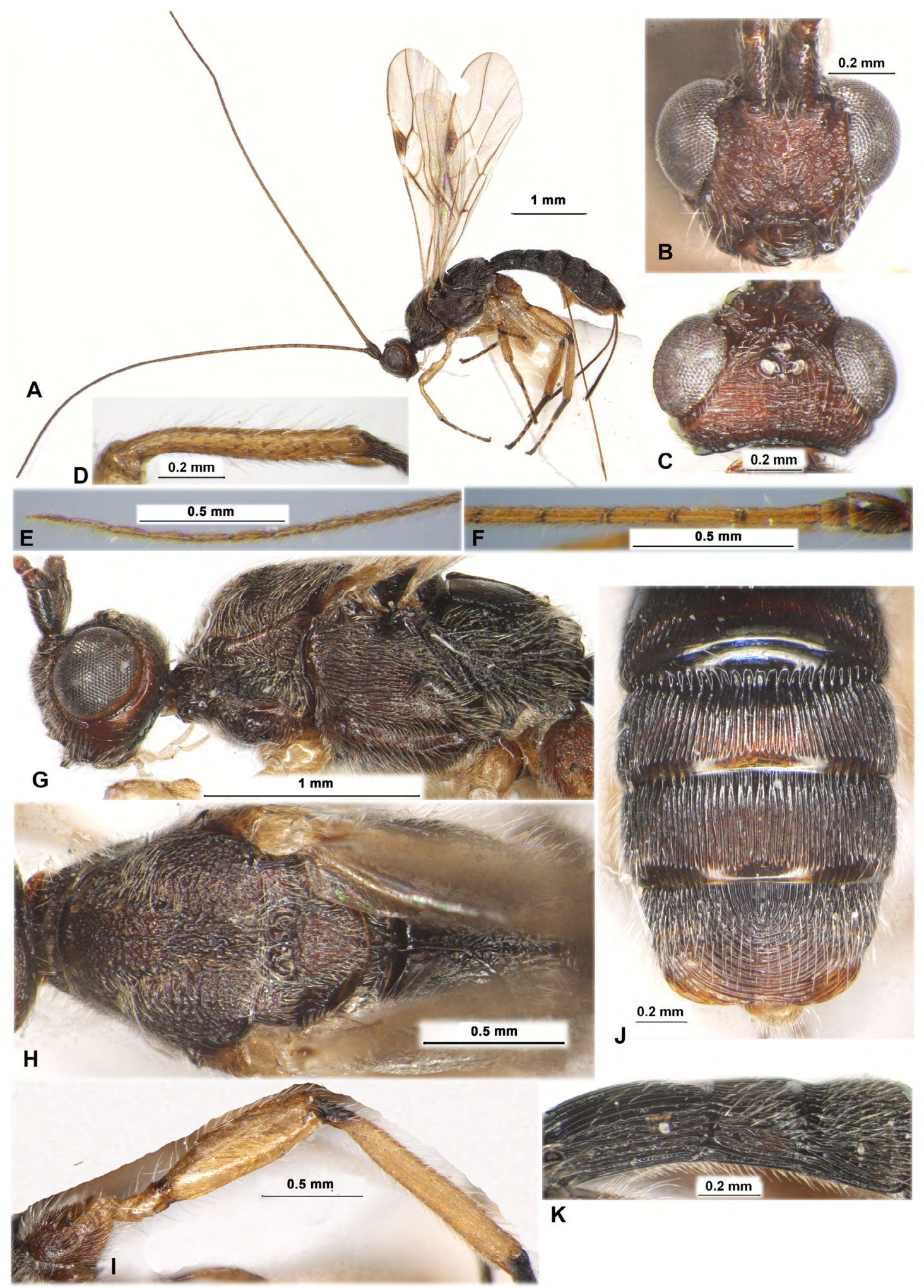

K

Fig. 24. Mimipodoryctes rubriceps (Cameron, 1909),, , holotype of M. robustus Belokobylskij, 2000, ZISP. A. Habitus, lateral view. B. Head, front view. C. Head, dorsal view. D. Fore tibia, lateral view. E. Apical segments of antenna. F. Basal segments of antenna. G. Head and mesosoma, lateral view. H. Mesosoma, dorsal view. I. Hind leg, lateral view. J. Apical metasomal tergites, dorsal view. K. First to third metasomal tergites, lateral view. 
BELOKOBYLSKIJ S.A. \& ZALDÍVAR-RIVERÓN A., Reclassification of the Rhaconotini
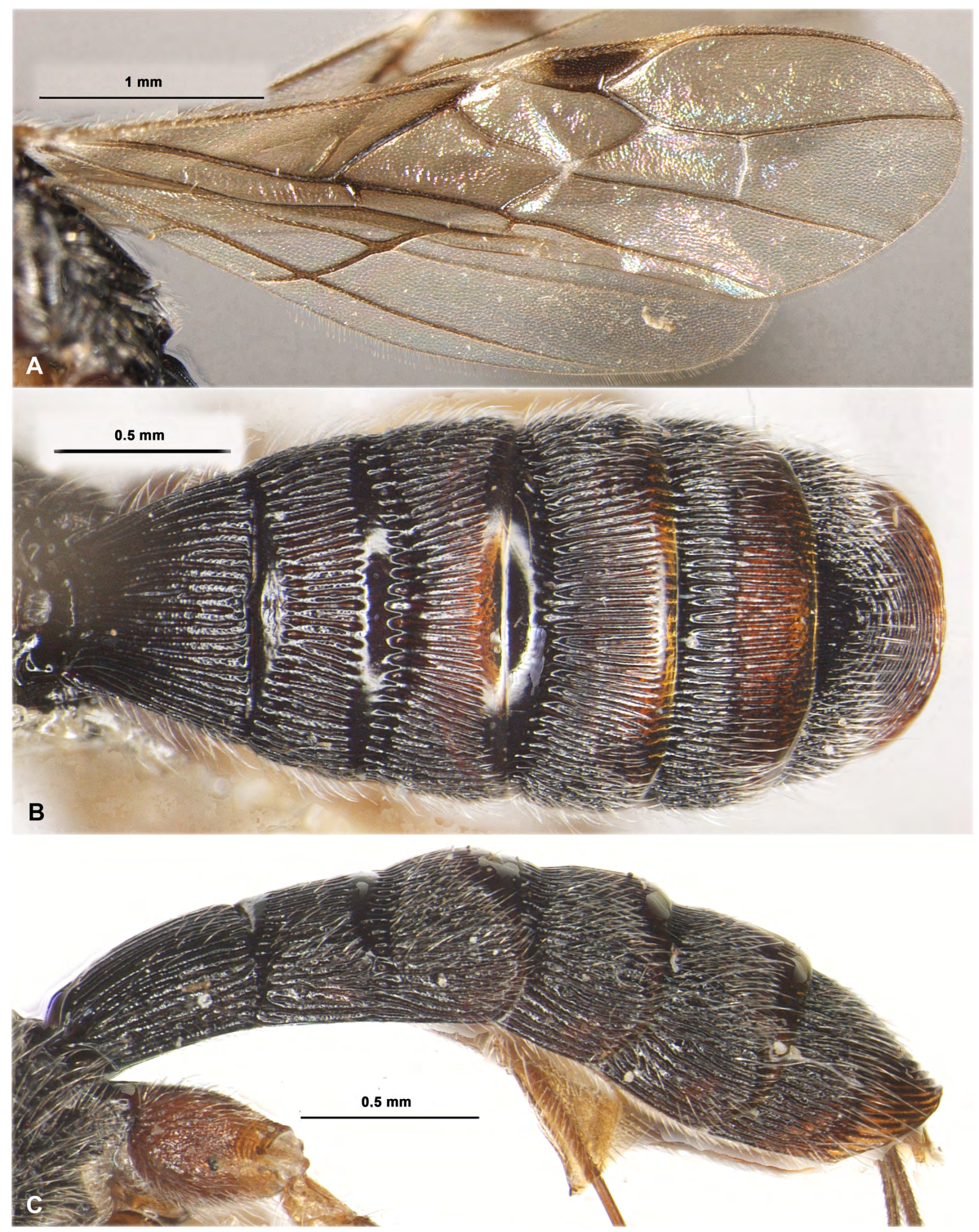

Fig. 25. Mimipodoryctes rubriceps (Cameron, 1909), , , holotype of M. robustus Belokobylskij, 2000, ZISP. A. Wings. B. Metasoma, dorsal view. C. Metasoma, lateral view. 


\section{Material examined}

VIETNAM • 1 q, holotype of M. robustus; "Vietnam, prov. Ha Son Binh, Mai Chau, forest, 31.X.1990, Belokobylskij"; ZISP • 1 ภे, paratype of M. robustus; "Vietnam, prov. Binh Phu, Tam Dao, 700 m, pines, 14.XI.1990, Belokobylskij"; ZISP.

\section{Description}

\section{Female}

MEASUREMENTS. Body length $4.1 \mathrm{~mm}$; fore wing length $3.9 \mathrm{~mm}$.

HEAD. Width 1.6 times its median length, 1.15 times width of mesoscutum. Temple behind eyes (dorsal view) weakly convex anteriorly, strongly and almost linearly narrowed posteriorly. Transverse diameter of eye 2.4 times as long as temple. Ocelli medium-sized, arranged in obtuse triangle with base 1.25 times its sides; POL almost equal to OD, 0.5 times OOL. Eye weakly emarginated opposite antennal sockets, 1.2 times as high as broad. Malar space 0.4 times height of eye, almost equal to basal width of mandible. Face width equal to eye height and 1.1 times height of face and clypeus combined. Upper margin of clypeus situated almost on same level as lower level of eyes. Width of hypoclypeal depression 0.7 times distance from depression to eye, 0.35 times width of face. Vertex weakly convex. Head below eyes (front view) distinctly and almost linearly narrowed Antennae weakly setiform, more than 38-segmented (apical segments missing). Scapus 1.8 times as long as its maximum width, 3.2 times length of pedicel. First flagellar segment 4.3 times as long as its apical width, 1.15 times as long as second segment. Subapical segments 4.5 times as long as their width.

Mesosoma. Length 1.9 times its height. Pronotal carina distinct, situated submedially. Mesoscutum (dorsal view) about as long as wide. Notauli rather deep in anterior half and shallow in posterior half, rather wide, distinctly crenulate-rugose. Median lobe of mesoscutum convex anteriorly, without median longitudinal furrow. Prescutellar depression with high median carina, sparsely and distinctly rugose, weakly curved along posterior margin, 0.3 times as long as scutellum. Scutellum almost as long as its maximum width. Subalar depression coarsely longitudinally striate. Sternaulus almost smooth. Prepectal carina below with very low lobes opposite fore coxae. Metanotum dorso-medially with two high and curved lateral and distinct median carinae (dorsal view). Propodeum weakly arcuately sloping backward (lateral view). Metapleural flange posteriorly with very dense white pubescence.

Wings. Length of fore wing 3.4 times its maximum width. Pterostigma 3.6 times as long as wide. Metacarpus (1-R1) 1.4 times as long as pterostigma. Radial (marginal) cell 3.5 times as long as wide. Radial vein (r) arising from middle of pterostigma. First radial abscissa (r) forming almost straight line with second abscissa (3-SR). Second radial abscissa (3-SR) 1.9 times as long as first abscissa (r), 0.45 times as long as the almost straight third abscissa (SR1), about as long as second radiomedial vein ( $\mathrm{r}-$ m). Second radiomedial (submarginal) cell medium size, very weakly widened apically, its length 2.8 times maximum width, 1.15 times length of the narrow brachial (subdiscal) cell. First medial abscissa $(1-\mathrm{SR}+\mathrm{M})$ distinctly sinuate. Second abscissa of medial vein $(2-\mathrm{SR}+\mathrm{M})$ short. Recurrent vein $(\mathrm{m}-\mathrm{cu})$ 0.85 times as long as first radiomedial vein (2-SR). Mediocubital vein (M+CU1) very weakly curved in apical half. Distance from nervulus (cu-a) to basal (1-M) vein 0.7 times nervulus (cu-a) length. Brachial (subdiscal) cell linearly closed distinctly behind recurrent vein; posterior bulla present but short; posterior abscissa of anal vein (2-1A) (behind brachial vein (CU1b)) present but short. Hind wing 4.7 times as long as wide. Recurrent vein (m-cu) weakly curved, oblique towards base of wing.

LEGS. Hind coxa 1.4 times as long as wide (with tooth). Hind femur about 3.0 times as long as wide. Hind tarsus as long as the thick hind tibia. Hind basitarsus with distinct lower keel and with dense pale setosity. Second segment of hind tarsus 0.5 times as long as basitarsus, almost as long as fifth segment (without pretarsus). 
Metasoma. Length about as long as head and mesosoma combined, with sixth visible tergites, remaining segments almost not protruding behind sixth tergite. First tergite distinctly, almost linearly and evenly widened from base to apex. Maximum width of first tergite almost 2.0 times its minimum basal width; length of first tergite 0.8 times its apical width, 0.9 times length of propodeum. Basal area of second tergite 0.4 times as long as remaining tergite; apical area 0.6 times as long as remaining tergite. Median length of second tergite (with areas) 0.6 times its basal width, 1.4 times median length of third tergite. Posterior margin of sixth tergite weakly and widely emarginated medially, without postero-ventral lobes. Sixth tergite 1.1 times as long as fifth tergite, 1.4 times as long as fourth tergites. Ovipositor sheath 0.8 times as long as metasoma, 1.15 times as long as mesosoma, 0.55 times as long as fore wing.

SCULPTURE AND PUBESCENCE. Vertex coarsely undulately transversally striate, with additional dense rugosity between striae; frons with coarse oblique and irregular striae. Face entirely rugose-striate; temple smooth in anterior half, partly subvertically striate with rugosity in posterior half, with coarse short rugae along occipital carina. Mesonotum entirely coarsely transverse-curvedly striate with dense rugulosity, partly with granulation between rugae. Scutellum entirely rugulose-granulate. Mesopleuron distinctly longitudinally striate in upper two-thirds, smooth posteriorly and below sternaulus. Metapleuron entirely coarsely rugose-reticulate. Propodeum with large, smooth to coriaceous and shortly rugose along carinae basolateral areas; remaining propodeum coarsely rugose-reticulate. Hind coxa densely transverse striate, with fine additional rugulosity and granulation, almost smooth or smooth in ventral half. Hind femur densely striate dorsally, granulate-coriaceous laterally, almost smooth below. First tergite entirely, second tergite mostly (except smooth basal and apical areas), and third-fifth tergites mostly (except smooth posterior $0.20-0.25$ ) longitudinally striate and with fine rugulosity between striae. Sixth tergite entirely densely semi-circularly striate, with additional rugulosity between striae in basal half. Secondsixth tergites laterally densely longitudinally striate, with fine rugulosity between striae. Vertex entirely with densely semi-erect pale setae. Mesonotum entirely with very dense, short and semi-erect yellow setae. Mesopleuron entirely in dense and mixed (short and long) setae. Hind tibia dorsally with dense, numerous and rather long semi-erect short and long setae, length of long setae 0.6-0.8 times maximum width of tibia. Laterotergites of metasoma with dense and rather long pale setae.

Colour. Body black, head dark red or dark reddish brown, infuscate posteriorly, mesothorax and distal half of sixth metasomal tergite with reddish tint. Antenna light reddish brown in basal half, two basal segments and apical half of antenna dark reddish brown to black. Palps pale yellow. Legs yellow to reddish yellow, hind coxa light reddish brown, hind tibia basally and all tarsi (except yellow apical $0.2-0.3$ of first-fourth segments) black or dark brown. Ovipositor sheath dark brown to black. Fore wing distinctly infuscate. Pterostigma dark brown, yellow in basal and apical 0.25.

\section{Male}

Measurements. Body length 2.8-3.9 mm; fore wing length $2.1-2.7 \mathrm{~mm}$.

LEGS. Length of hind femur 2.7-3.0 times its width.

Metasoma. First and second metasomal tergites mobile connected. First tergite $0.9-1.1$ times as long as its apical width. Second tergite 0.7 times as long as its basal width. Apical area of second tergite narrow, $0.2-0.3$ times as long as remaining tergite. Metasoma with seven visible tergites. Seventh tergite short and rather narrow, strongly semi-circularly striate. Otherwise similar to female.

\section{Distribution}

Indonesia, Malaysia, Papua New Guinea, Thailand, Vietnam. 
Genus Neorhaconotus Belokobylskij, Iqbal \& Austin, 2004

Neorhaconotus Belokobylskij, Iqbal \& Austin, 2004: 65.

Neorhaconotus - Belokobylskij 2005: 205. — Belokobylskij \& Austin 2013: 341. — Yu et al. 2016. — Jasso-Martínez et al. 2019: 165.

\section{Type species}

Neorhaconotus rieki Belokobylskij, Iqbal \& Austin, 2004, by original designation (Belokobylskij et al. 2004).

\section{Description (Figs 26-27)}

HEAD. Head transverse. Ocellar arranged in obtuse triangle with base 1.2 times its sides. Frons slightly concave, without median keel, but with distinct curved carina along eyes. Oblique furrow between eye and antennal socket absent. Eye glabrous or with sparse and very short setae, with shallow or very shallow emargination opposite antennal sockets. Distance between antennal sockets wide. Malar suture absent. Clypeal suture distinct and complete. Hypoclypeal depression weakly oval. Postgenal bridge narrow. Occipital carina strong and complete, fused below with hypostomal carina upper base of mandible. Maxillary palps 6-segmented; labial palps 4-segmented, third labial segment long. Scapus of antenna long and wide, not flattened laterally, without apical lobe and basal constriction; length of scapus 1.7-2.0 times its maximum width. First flagellar segment straight, almost as long as second segment.

Mesosoma. Mesosoma not depressed dorso-ventrally. Neck of prothorax long, almost flat dorsally, with distinct pronotal keel, with long smooth anterior lobe which is marginated subapically. Postero-dorsal propleural lobe distinct and wide. Mesonotum subvertical or obliquely curving and distinctly elevated above pronotum. Median lobe of mesoscutum without anterolateral corners. Notauli complete, entirely shallow or sometimes deeper in anterior half and distinctly shallow in posterior half. Prescutellar depression long, rugulose, with five-seven distinct carinae. Metanotum with distinct, obtuse or pointed median tooth (lateral view). Subalar depression shallow, wide and coarsely sculptured. Mesopleural pit present, elongate and deep. Sternaulus (precoxal sulcus) deep, long, almost straight, weakly or distinctly sculptured. Prepectal carina strong. Prepectus with or without lateral carinae. Postpectal carina absent. Metapleural flange long, narrow and densely pubescent at least partly. Propodeum with long areola and basolateral areas distinctly delineated by carinae; with more or less distinct tubercles or without them; propodeal bridge absent

LEGS. Fore tibia with several slender spines arranged in single row. Hind coxa with distinct basoventral corner and tubercle. Fore and middle femora with weak medio-dorsal protuberances, virtually missing on hind femur. Hind femur rather thick, 3.0-3.8 times as long as wide. Inner hind tibial spur 0.25-0.35 times as long as basitarsus. Hind basitarsus $0.6-0.7$ times as long as second-fifth segments combined.

WINGS. Fore wing usually large, but sometimes micropterous. Radial vein (r) arising almost from middle of pterostigma. Radial (marginal) cell not shortened. Both radiomedial veins (2RS, r-m) present. Second radiomedial (submarginal) cell long or median sized. Discoidal (discal) cell petiolate. Recurrent vein (m-cu) postfurcal. Nervulus (cu-a) distinctly postfurcal. Parallel vein (CU1a) interstitial. Brachial (first subdiscal) cell closed apically before recurrent vein (m-cu). Both transverse anal veins $(2 \mathrm{~A}, \mathrm{a})$ absent. Mediocubital vein $(\mathrm{M}+\mathrm{CU})$ entirely sclerotised. Hind wing with three hamuli. Second costal abscissa $(\mathrm{SC}+\mathrm{R})$ and nervulus (cu-a) present. Medial (basal) cell closed anteriorly, distinctly widened distally, its length 6.5-7.0 times maximum width. Radial vein (RS) unsclerotised, without additional transverse 
vein (r). Submedial (subbasal) cell long; first mediocubital abscissa $(\mathrm{M}+\mathrm{CU})$ 0.85-1.0 times as long as second abscissa $(1 \mathrm{M})$. Recurrent vein $(\mathrm{m}-\mathrm{cu})$ present, oblique, curvedly directed to base of wing.

Metasoma. Metasoma with five dorsally visible tergites. First tergite short and wide, with small dorsope; its spiracular tubercles small and situated in basal quarter of tergite; dorsal carinae fine and subparallel. Acrosternite of first segment short, $0.15-0.20$ times as long as first tergite. First and second tergites movably connected, not fused. Second suture deep, wide and sometimes weakly curved laterally. Second tergite without any furrows or defined areas, shorter than third tergite. Third tergite with deep and weakly curved transverse crenulate furrows in basal $0.3-0.5$. Second to fifth tergites with separate laterotergites. Fifth tergite slightly enlarged, 1.2-1.6 times as long as fourth tergite, almost concealing the succeeding tergites. Fourth to sixth tergites with transverse subapical rows of long sparse erect setae. Ovipositor slightly longer or shorter than metasoma; apex of its dorsal valve with two distinct and widely separated nodes, apex of ventral valve serrate.

\section{Diagnosis}

Neorhaconotus is similar to Rhaconotus, but differs from the latter genus by having the submedial (subbasal) cell of the hind wing large (vs distinctly short) and first mediocubital abscissa $(\mathrm{M}+\mathrm{CU})$ 0.85-1.0 times as long as second abscissa ( $1 \mathrm{M})$ (vs $0.3-0.5$ times), the propodeum with elongate areola distinctly delineated by high carinae (vs without areola), the posterior metasomal tergites (started from fourth) mostly smooth (vs mostly sculptures at least basally), and tergites behind fifth one are shortly projecting (vs almost never projected).

\section{Composition}

Neorhaconotus cardaleae Belokobylskij, Iqbal \& Austin, 2004 (AU); N. julieae Belokobylskij \& Austin, 2013 (AU); N. pappi Belokobylskij, Iqbal \& Austin, 2004 (AU); N. rieki Belokobylskij, Iqbal \& Austin, 2004 (AU).

\section{Hosts}

Unknown.

\section{Distribution}

Australasian region.

\section{Remarks}

A key to the described macropterous species of Neorhaconotus was published by Belokobylskij et al. (2004: 66).

Neorhaconotus rieki Belokobylskij, Iqbal \& Austin, 2004

Figs 26-27

Neorhaconotus rieki Belokobylskij, Iqbal \& Austin, 2004: 67.

Neorhaconotus rieki - Belokobylskij \& Austin 2013: 344. — Yu et al. 2016.

\section{Material examined}

Holotype

AUSTRALIA • + ; “12 N Norseman, 25 Nov 1958, E.F. Riek, W.A.”; ANIC.

\section{Distribution}

Australia (Western Australia Province). 




Fig. 26. Neorhaconotus rieki Belokobylskij, Iqbal \& Austin, 2004, + , holotype, ANIC. A. Habitus, lateral view. B. Head, front view. C. Body, dorsal view. D. Body, lateral view. Image copyright of Dr. Olivia Evangelista, Australian National Insect Collection (ANIC-CSIRO). 

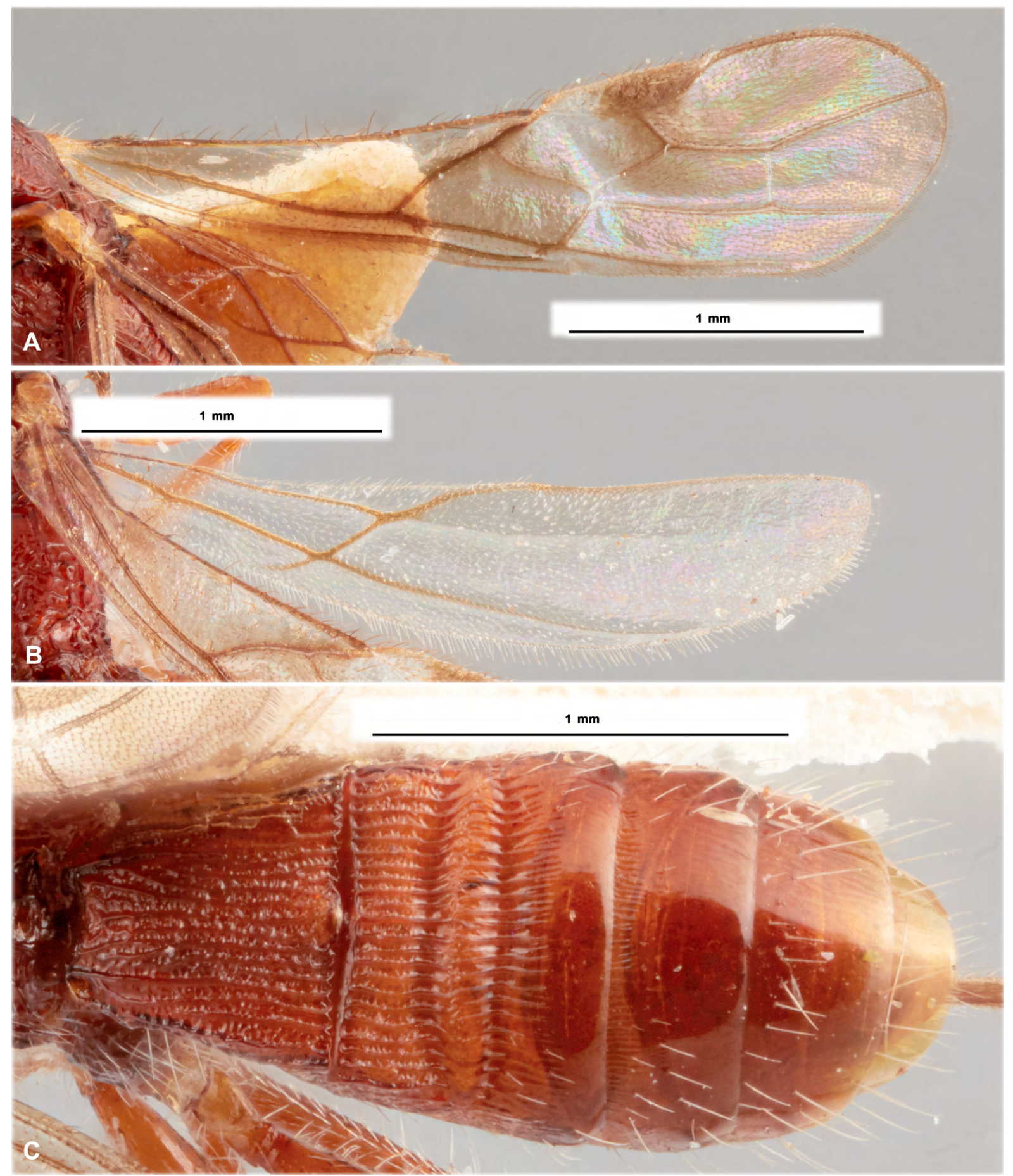

Fig. 27. Neorhaconotus rieki Belokobylskij, Iqbal \& Austin, 2004,, , holotype, ANIC. A. Fore wing. B. Hind wing. C. Metasoma, dorsal view. Image copyright of Dr. Olivia Evangelista, Australian National Insect Collection (ANIC-CSIRO). 


\section{Remarks}

The description of this species was based on a single specimen (holotype) (Belokobylskij et al., 2004). We have not studied any new additional specimen for description of morphological variation of $N$. rieki.

Genus Platyspathius Viereck, 1911

Platyspathius Viereck, 1911: 185.

Spathiohormius Enderlein, 1912: 21 (type species Spathiohormius ornatulus Enderlein, 1912, by original designation).

Platyspathius - Nixon 1943: 423. - Granger 1949: 153. — Shenefelt \& Marsh 1976: 1384. Belokobylskij 1996a: 178. — Belokobylskij \& Ku 2001: 37. — van Achterberg 2003: 283 Belokobylskij et al. 2004: 81. — Belokobylskij \& Maeto 2006: 735; 2009: 402. — Belokobylskij \& Villemant 2015: 113. — Yu et al. 2016. - Jasso-Martínez et al. 2019: 165.

Spathiohormius - Shenefelt \& Marsh 1976: 1384 (as synonym of Platyspathius).

\section{Type species}

Platyspathius pictipennis Viereck, 1911.

\section{Description (Figs 28-37)}

HEAD. Head usually not depressed, high, rather transverse. Vertex entirely very densely granulate. Ocelli arranged in obtuse triangle. Frons weakly concave, without median keel, usually with very fine longitudinal furrow. Eyes often with very short and sparse setae. Occipital carina dorsally complete, obliterate below and not joining ventrally with hypostomal carina. Malar suture absent. Clypeal suture rather distinct and complete. Hypoclypeal depression medium size, round. Palps rather short; maxillary palps 6-segmented, its sixth (apical) segment longer than fifth segment; labial palps short, 4-segmented, its third segment not shortened. Scapus wide, rather short, without apical lobe and basal constriction, its ventral margin (lateral view) shorter than dorsal margin. First flagellar segment subcylindrical, very weakly curved, almost as long as or weakly longer second segment. Apical segment pointed apically and without spine.

Mesosoma. Mesosoma not depressed dorso-ventral and relatively short. Neck of prothorax short. Pronotum dorsally distinctly convex, with fine and submedially situated pronotal carina; pronope absent. Propleural dorsoposterior flange long and narrow. Mesonotum relatively highly and roundly elevated above pronotum, mostly densely and distinctly granulate-coriaceous. Median lobe of mesonotum with very shallow median longitudinal furrow, without anterolateral corners. Notauli incomplete, deep in anterior half and absent or almost absent in posterior half. Tegula short, widened distally, weakly convex along its posterior margin. Prescutellar depression short, distinctly curved, with several carinae. Lateral longitudinal wing-like flanges on the level of prescutellar depression narrow and low. Scutellum more or less distinctly convex, often with lateral carinae. Metanotum with short, wide and pointed apically median tooth (lateral view). Sternaulus (precoxal sulcus) very shallow, narrow, long, running along anterior $0.5-0.6$ of lower part of mesopleuron, more or less straight. Prepectal carina distinct and complete, rather wide below. Postpectal carina absent. Metapleural flange rather long, narrow, rounded apically. Propodeum without areas; lateral tubercles present, but low; propodeal bridge absent. Propodeal spiracles small and round.

Wings. Pterostigma of fore wing rather narrow and long. Radial (marginal) cell not shortened. Both radiomedial veins (2-SR, r-m) present. Second radiomedial (submarginal) cell long and rather wide. Recurrent vein $(\mathrm{m}-\mathrm{cu})$ postfurcal or rarely subinterstitial. Discoidal (discal) cell petiolate anteriorly, petiole (1-SR) long or short. Nervulus (cu-a) almost interstitial or postfurcal, rarely weakly antefurcal, 
oblique towards base of wing. Brachial (subdiscal) cell weakly narrowed apically, gently curvedly closed apically usually before recurrent vein $(\mathrm{m}-\mathrm{cu})$, without antero-posterior corner. Parallel vein (CU1a) always interstitial. Transverse anal veins (2A, a) absent. Hind wing with three hamuli. Radial vein (SR) arising from costal vein (2-SC+R) far separating from basal vein (1r-m). Radial (marginal) cell without transverse vein (r). Medial (basal) cell weakly widened towards apex from base. Nervellus (cu-a) present. Submedial (subbasal) cell short; first abscissa of mediocubital vein (M+CU) 0.5-0.6 times as long as second abscissa $(1-\mathrm{M})$. Recurrent (m-cu) vein long, curved, strongly oblique towards base of wing.

LEGS. Fore and middle tibiae with distinct thickened spines arranged in narrow stripe. All tibiae thick. Middle tarsal segments long. Hind coxa short, wide, subrounded, without basoventral corner, often without basoventral tubercle or sometimes present but small. Inner spur of hind tibia simple, 0.3 times as long as hind basitarsus. Hind femur wide, without distinct dorsal protuberance. Hind basitarsus 0.6 times as long as second-fifth segments combined. Claws short and simple.

Metasoma. First tergite semi-petiolate, long, rather narrow, very densely and very small areolatepunctate. Acrosternite of first tergite weakly elongate, about $0.35-0.40$ times as long as first tergite, its apical margin situated about on or weakly behind level of spiracles. Dorsope of first tergite shallow and rather small; present wide basolateral processes; spiracular tubercles often absent, spiracles situated almost in basal 0.3 of tergite; dorsal carinae absent, but present distinct and complete lateral carinae. Second tergite without basal and often without apical areas delineated by furrows. Second suture usually shallow or very shallow, rather narrow or sometimes wide, complete and evenly curved. Third tergite without transverse depression or furrow. Second and third tergites mainly very densely and very small areolate-punctate. Second to sixth tergites with distinctly separated laterotergites. Six-seven dorsally visible tergites. Fifth tergite weakly enlarged, slightly longer than previous tergite (measured from transverse subbasal furrow), not covered following apical segments. Setae on fourth-sixth tergites widely distributed laterally and almost in single submedian line medially.

\section{Diagnosis}

Platyspathius characterise by having the following combination of diagnostic morphological features: acrosternite of first metasomal segment distinctly (but not strongly) elongate; first tergite usually long and narrow; sculpture of vertex and mesoscutum densely and sometimes finely granulate-areolate; firstthird metasomal tergites mainly or entirely densely areolate-reticulate; hind coxa without basoventral corner and tubercle; all tibiae (especially posterior) thickened; propodeum without any delineated areas, parallel vein (CU1a) of fore wing mostly interstitial, recurrent vein (m-cu) always postfurcal, and metasoma with more than five dorsally visible tergites with usually enlarged fifth tergite.

\section{Composition}

This genus currently consists of two subgenera, Lenticularia van Achterberg, 2003 and Platyspathius s. str. The following species belong to the nominative subgenus: Platyspathius (Platyspathius) bisignatus (Walker, 1860) (OR); P. (P.) clymene Nixon, 1943 (AF); P. (P.) dice Nixon, 1943 (AF); P. (P.) europaeus van Achterberg, 2003 (PA); P. (P.) hospitus Belokobylskij \& Ku, 2001 (PA); P. (P.) ornatulus (Enderlein, 1912) (P. dinoderi Gahan, 1925) (PA, OR, PC); P. (P.) picardi Belokobylskij \& Villemant, 2015 (PA); P. (P.) pictipennis Viereck, 1911 (AF); P. (P.) pyrene Nixon, 1943 (OR); P. (P.) ruiliensis Chao, 1978 (OR); P. (P.) thyone Nixon, 1943 (OR); P. (P.) turneri Nixon, 1943 (AF); P. (P.) venezuelicus sp. nov. (NT). 


\section{Hosts}

Coleoptera: Dinoderus brevis Horn, 1878; D. minutus (Fabricius, 1775), D. ocellaris Stephens, 1830; Sinoxylon anale Lesne, 1897; S. senegalense Karsch, 1881 (Bostrichidae) (Yu et al. 2016); Anobium punctatum (DeGeer, 1774) (Anobiidae) (Belokobylskij \& Villemant, 2015).

\section{Distribution}

Afrotropical, Neotropical, Oceanic, Oriental and Palaearctic regions.

Platyspathius (Platyspathius) pictipennis Viereck, 1911

Figs 28-29

Platyspathius pictipennis Viereck, 1911: 185.

Rhaconotus spathulatus Szépligeti, 1914: 198 (HNHM; not examined).

Platyspathius pictipennis - Nixon 1943: 429. — Granger 1949: 153. — Shenefelt \& Marsh 1976: 1385. — van Achterberg 2003: 286.

Rhaconotus spathulatus - Nixon 1943: 429 (as synonym of P. pictipennis).

\section{Material examined}

\section{Holotype}

MOZAMBIQUE • \; "101", "CW Howard Collector", "Xalasi (Maputo)", "Lor Marquez, Africa, 4.VI.09”, “Type, No 13489, U.S.N.M.”, "Platyspathius pictipennis Vier., Type §’”; USNM.

\section{Description}

\section{Male}

Measurements. Body length $3.9 \mathrm{~mm}$; fore wing length $2.7 \mathrm{~mm}$.

HEAD. Width 1.5 times its median length, 1.2 times width of mesoscutum. Head behind eyes (dorsal view) weakly convex in anterior half and weakly roundly narrowed in posterior half. Transverse diameter of eye 1.15 times length of temple. Ocelli medium-sized, arranged in triangle with base 1.3 times its sides; POL 1.8 times OD, 0.7 times OOL. Eye with short and sparse setae, very weakly emarginated opposite antennal sockets, 1.3 times as high as broad. Malar space 0.65 times height of eye, 1.3 times basal width of mandible. Face convex, its width almost equal to height of eye and 1.2 times height of face and clypeus combined. Clypeus marrow, its upper margin situated distinctly below than lower level of eyes. Hypoclypeal depression round, its width 0.6 times distance from edge of depression to eye, 0.4 times width of face. Occipital carina not fused below with hypostomal carina being obliterated on wide distance. Vertex weakly convex. Head below eyes distinctly and roundly narrowed. Antennae rather thick, filiform, 25-segmented, weakly shorter than body. Scapus 1.5 times as long as its maximum width. First flagellar segment about 5.0 times as long as its apical width, 1.05 times as long as second segment. Penultimate segment 2.2 times as long as wide, 0.5 times as long as first segment, 0.8 times as long as apical segment.

Mesosoma. Length 2.0 times its height. Mesoscutum (dorsal view) 0.9 times as long as maximum width; its median lobe weakly convex anteriorly. Notauli present and crenulate in anterior 0.6 , completely absent in posterior 0.4. Prescutellar depression rather deep, short, distinctly and roundly curved posterolaterally, with seven distinct carinae, smooth between carinae, about 0.3 times as long as scutellum. Sternaulus (precoxal furrow) straight, finely reticulate-coriaceous. Prepectal carina below without widened lobes opposite fore coxae. Subalar depression finely crenulate with reticulation. 

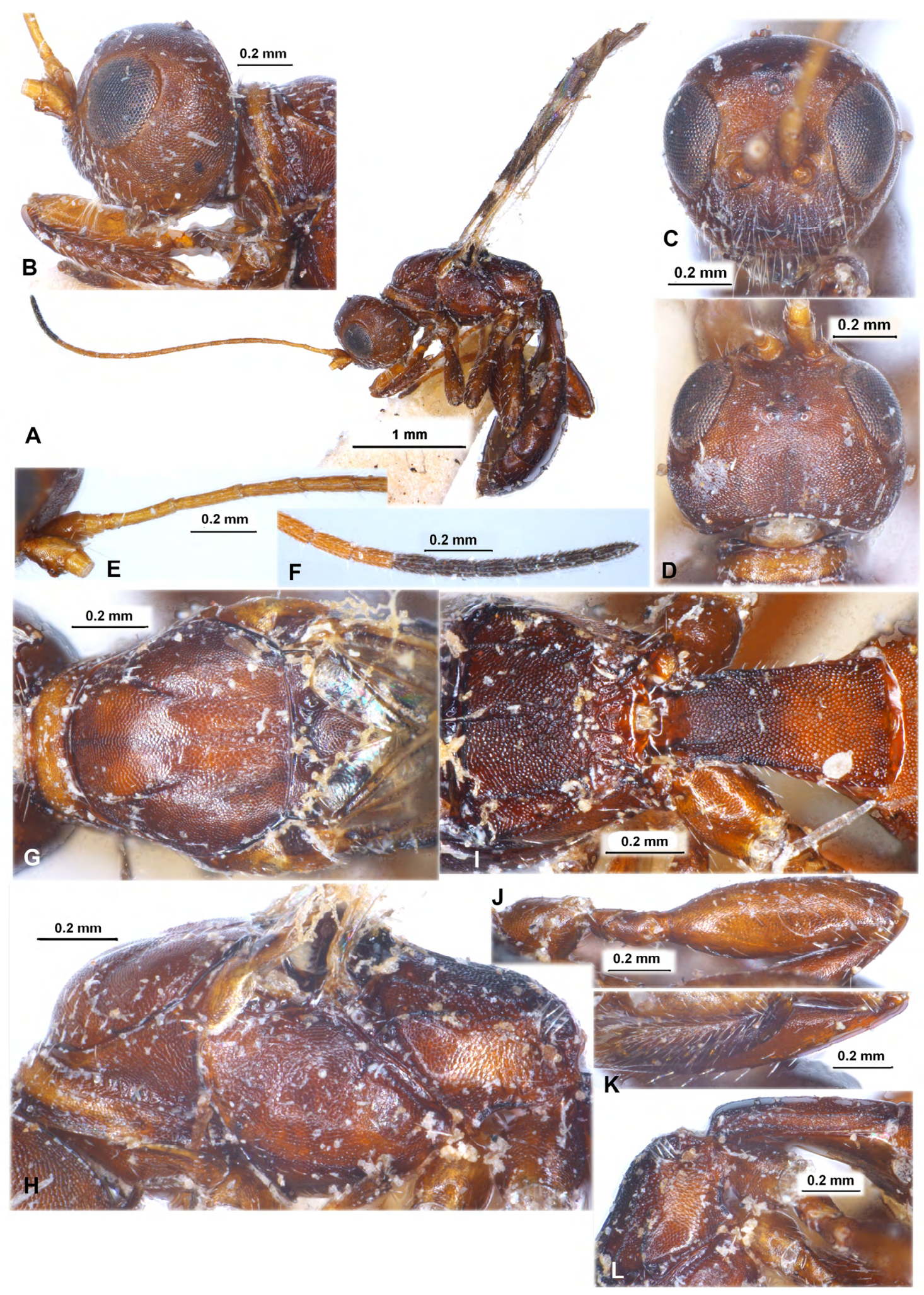

Fig. 28. Platyspathius (Platyspathius) pictipennis Viereck,1911, §̊, holotype, USNM. A. Habitus, lateral view. B. Head, lateral view. C. Head, frontal view. D. Head, dorsal view. E. Basal segments of antenna. F. Apical segments of antenna. G. Mesoscutum, dorsal view. H. Mesosoma, lateral view. I. Propodeum and first metasomal tergite, dorsal view. J. Hind coxa and femur, lateral view. K. Hind tibia, lateral view. L. Propodeum and first metasomal tergite, lateral view. 


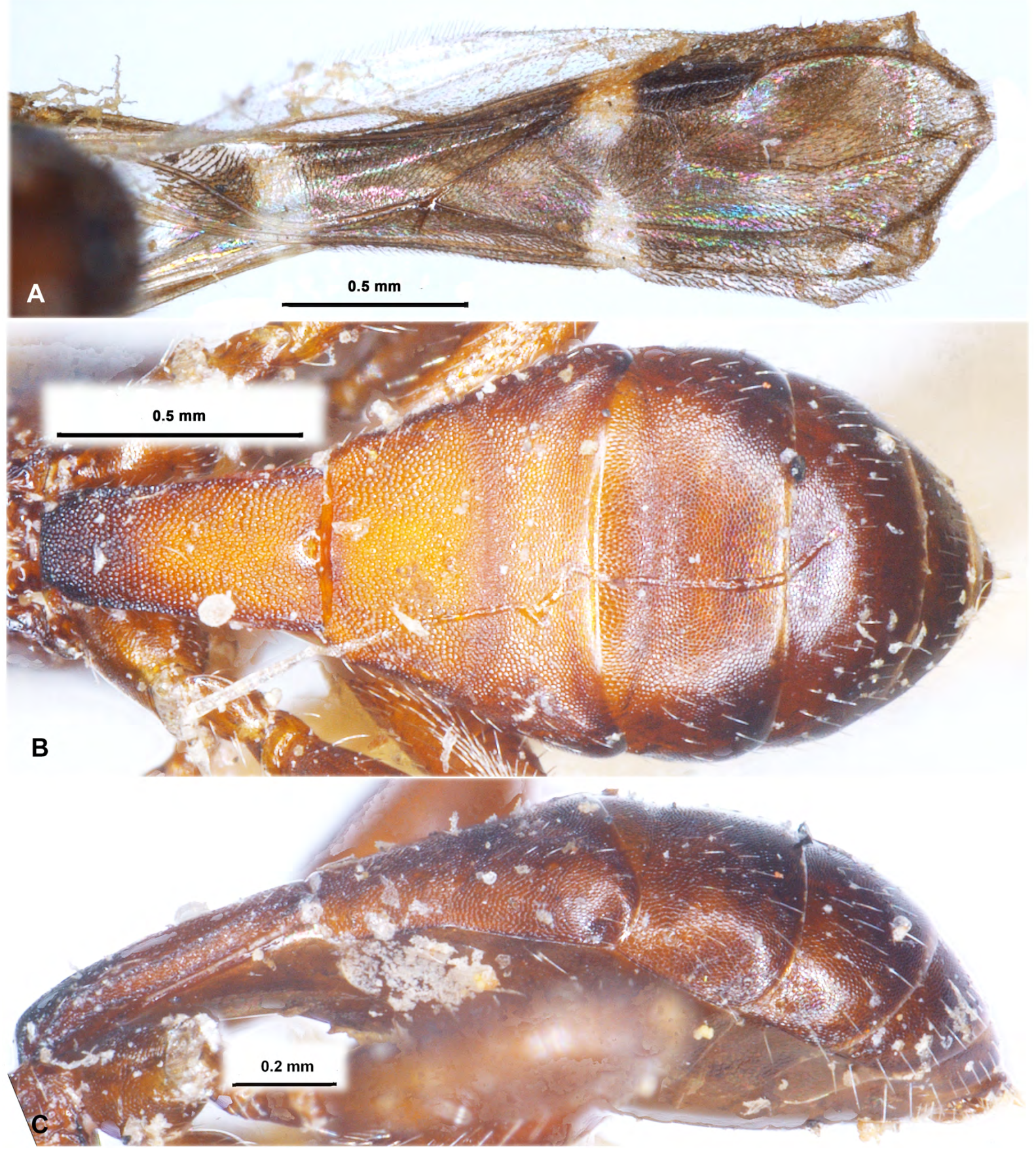

Fig. 29. Platyspathius (Platyspathius) pictipennis Viereck, 1911, Ô, holotype, USNM. A. Wings. B. Metasoma, dorsal view. C. Metasoma, lateral view. 
WINGS. Fore wing 4.3 times its maximum width. Radial (marginal) cell 2.9 times as long as maximum width. Metacarpus (1-R1) 1.1 times as long as pterostigma. First radial abscissa (r) forming obtuse angle with second abscissa (3-SR). Second radial abscissa (3-SR) 2.8 times as long as first abscissa (r), 0.75 times as long as the straight third abscissa (SR1), 1.55 times as long as first radiomedial vein (2-SR). Second radiomedial (submarginal) cell long, weakly narrowed distally, 3.3 times as long as wide, 1.3 times as long as the rather wide basally and narrowed apically brachial (subdiscal) cell. First medial abscissa (1-SR+M) weakly sinuate. Recurrent vein (m-cu) 1.3 times as long as second median abscissa $(2-\mathrm{SR}+\mathrm{M}), 0.35$ times as long as first radiomedial vein (2-SR). Mediocubital vein $(\mathrm{M}+\mathrm{CU} 1)$ strongly curved to anal vein (2-1A) in distal half. Nervulus (cu-a) interstitial. Brachial (subdiscal) cell closed shortly before level of recurrent vein; posterior bulla and posterior abscissa of anal vein (2-1A) (behind brachial vein (CU1b)) absent. Hind wing about 6.5 times as long as wide. Recurrent vein (m-cu) unsclerotised.

Legs. Fore femur about 3.0 times as long as wide. Hind coxa 1.3 times as long as wide. Hind femur almost 3.0 times its maximum width. Hind tibia with dense comb of long setae on inner apical margin. Hind tarsus 0.9 times as long as hind tibia. Second tarsal segment 0.45 times as long as basitarsus, 1.8 times as long as fourth segment, almost as long as fifth segment (without pretarsus).

Metasoma. Length as long as head and mesosoma combined. First tergite weakly and linearly widened towards apex; its maximum width 1.8 times basal width; its length 2.1 times apical width, 1.4 times length of propodeum. Median length of second tergite 0.85 times its basal width, 1.6 times length of third tergite. Second suture shallow, rather narrow, distinctly evenly curved. Fifth and sixth tergite straight on posterior margins, without median emargination and postero-ventral lobes. Six tergites rather distinctly protruding behind fifth tergite.

SCULPTURE AND PUBESCENCE. Vertex distinctly and very densely reticulate-granulate, partly with fine aciculation, without rugosity; frons densely rugulose-aciculate with dense granulation; face entirely, very densely and distinctly granulate with reticulation; temple entirely densely reticulate-granulate, partly upper and below with fine aciculation. Mesoscutum entirely densely and distinctly reticulate-coriaceous (granulation indistinct), finely rugulose on its wide medioposterior area, with distinct median carina in posterior 0.3 of mesoscutum. Scutellum densely and finely reticulate-coriaceous. Mesopleuron entirely finely or very finely and densely reticulate-coriaceous. Metapleuron finely and densely reticulatecoriaceous, additionally rugulose-striate in posterior quarter. Propodeum without areas, with distinct and almost complete lateral carinae and distinct median carina in basal half, entirely densely granulateareolate with sparse and distinct rugosity in posterior half. Hind coxa almost entirely densely and very finely reticulate-coriaceous or reticulate, without rugosity. Hind femur entirely finely or very finely reticulate or reticulate-coriaceous. First and second metasomal tergites with very dense and very small but distinct areolation, with complete and distinctly lateral carinae, with short dorsal carinae in basal 0.2 of first tergite. Second suture very densely and small areolate, without striation. Third and fourth tergites entirely rather finely and very densely small areolate-reticulate; fifth and sixth tergites entirely finely reticulate-coriaceous. Vertex almost entirely with short, sparse or very sparse and semi-erect pale setae, glabrous in wide median part. Mesoscutum mainly glabrous, with sparse, short and semi-erect setae arranged rather widely along notauli and in narrow row marginally. Mesopleuron widely glabrous medially. Hind tibia dorsally with rather short, sparse and semi-erect pale setae; length of these setae $0.2-0.3$ times maximum width of hind tibia.

CoLour. Body reddish brown to dark reddish brown, with light reddish brown areas laterally and dorsally, pronotum and head below brownish yellow or pale brown. Antenna yellow or brownish yellow, six apical segments contrasting black. Palps dark reddish brown. Legs light reddish brown, middle and hind coxae yellowish brown, tarsi (except dark apical segments) light reddish brown of brownish 
yellow, hind tibia basally dark, not paler than remaining part of tibia. Fore wing strongly darkened, less strongly apically, with three hyaline transverse stripes: wide basally (with very short and sparse setae), narrow on middle of basal half (crossing narrowest part of submedian area), narrow and interrupted medially on level of base of pterostigma. Pterostigma mostly dark brown, yellow in its basal 0.3.

\section{Distribution}

Cameroon, Democratic Republic of Congo, Madagascar, Mozambique, South Africa, Sudan, Uganda.

Platyspathius (Platyspathius) venezuelicus sp. nov. urn:1sid:zoobank.org:act:9151CADC-510B-4010-B0E4-96F878ED791E

Figs $30-31$

\section{Etymology}

Named after Venezuela, the country where the type material was collected.

\section{Material examined}

\section{Holotype}

VENEZUELA • +; “Venezuela, Estado de Lara, San Javier, 23.V.2006, CNIN 1192”; IB-UNAM.

\section{Paratypes}

VENEZUELA • 1 \%; same collection data as for holotype, but "CNIN 1627"; ZISP • 1 \%; same collection data as for holotype, but "CNIN 1628"; IB-UNAM • 1 q (metasoma missing); "Venezuela, Estado de Lara, San Javier, Taracuya, 2006”; IB-UNAM.

\section{Description}

\section{Female}

MEASUREMENTs. Body length 2.5-3.9 mm; fore wing length 1.6-2.3 mm.

HEAD. width (dorsal view) 1.3-1.5 times median length, 1.2-1.3 times maximum width of mesoscutum. Head behind eyes (dorsal view) evenly and weakly roundly narrowed; transverse diameter of eye 1.2-1.4 times as long as temple. Ocelli small, arranged in triangle with base 1.2-1.3 times its sides. POL 1.5-1.7 times OD, 0.4-0.5 times OOL. Eye glabrous, 1.15-1.25 times as high as broad. Malar space $0.55-0.70$ times height of eye, 1.1-1.3 times basal width of mandible. Face width $1.15-1.25$ times height of eye and 1.2-1.3 times height of face and clypeus combined. Clypeal suture distinct, but shallow, with additional weak carina. Hypoclypeal depression rather small and round, its width 0.5 times distance from edge of depression to eye, $0.30-0.35$ times width of face. Occipital carina complete dorsally, ventrally not joined with hypostomal carina being obliterated before base of mandible. Postgenal bridge rather wide. Head below eyes (front view) distinctly and weakly-roundly narrowed. Antennae slender, filiform, densely and shortly setose, more than 22-segmented (apical segments missing). Scapus 1.6-1.8 times as long as its maximum width. First flagellar segment 5.5-5.8 times as long as apical width, almost as long as or weakly longer than second segment. Subapical segments 2.8-3.0 times as long as their width.

Mesosoma. Length of mesosoma 2.0-2.1 times its height. Pronotal keel present, distinct, situated near middle of dorsal side of pronotum. Pronotal lateral oblique depressions narrow, rather shallow, densely coriaceous, anteriorly with fine crenulation, posteriorly with short longitudinal striae. Mesoscutum (lateral view) distinctly, uniformly and roundly elevated above pronotum. Notauli narrow, distinct in anterior $0.5-0.7$ and shallow to very shallow in posterior $0.3-0.5$, usually complete, anteriorly crenulate-coriaceous, only finely coriaceous posteriorly. Prescutellar depression short and curved, with four fine or distinct lateral and distinct median carinae, coriaceous between carinae, $0.25-0.30$ times as 


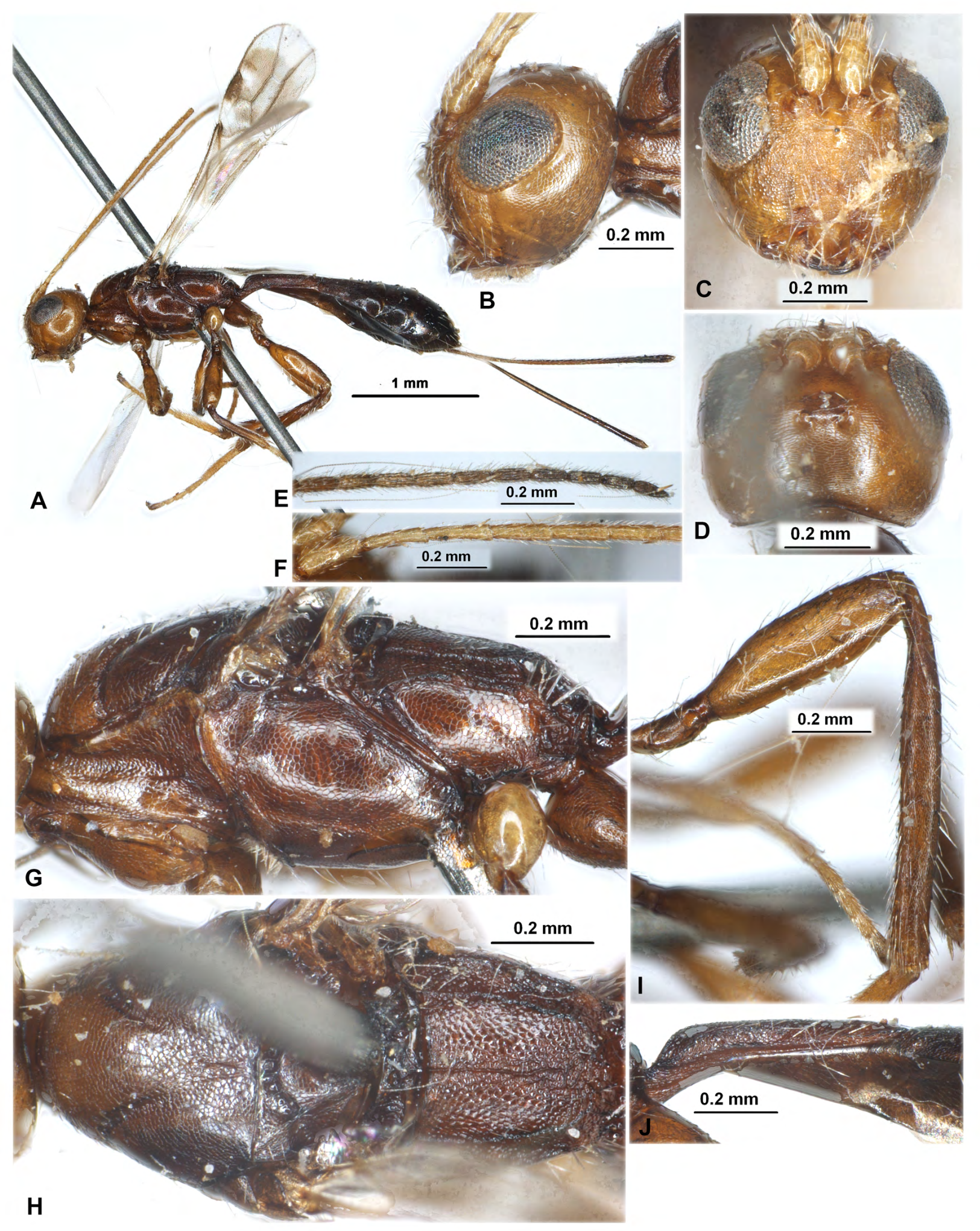

Fig. 30. Platyspathius (Platyspathius) venezuelicus sp. nov.,, , holotype, IB-UNAM. A. Habitus, lateral view. B. Head, front view. C. Head, dorsal view. D. Head, lateral view. E. Basal segments of antenna. F. Mesosoma, dorsal view. G. Mesosoma, lateral view. H. Propodeum and first metasomal tergite, dorsal view. I. First metasomal tergite, lateral view. 

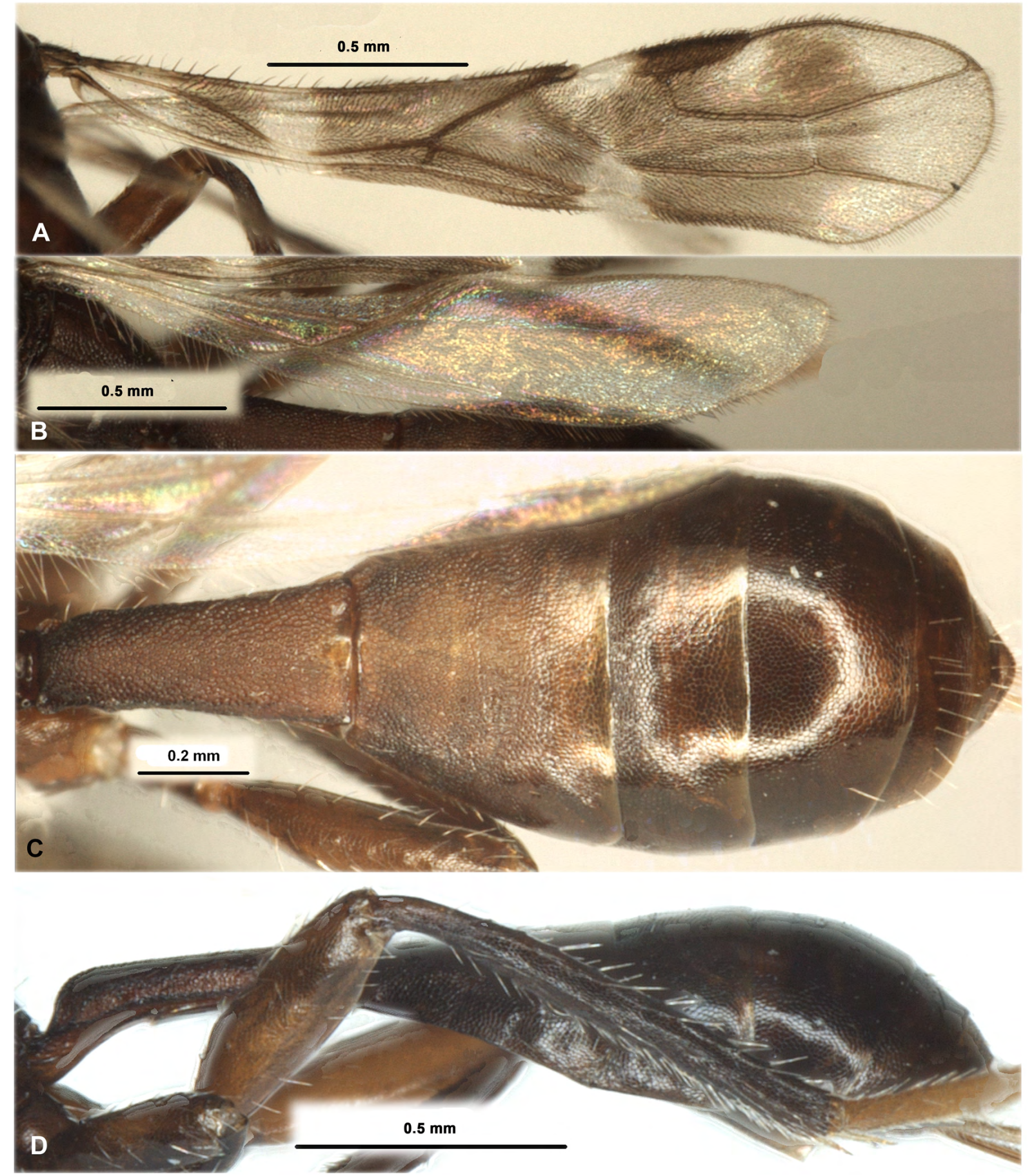

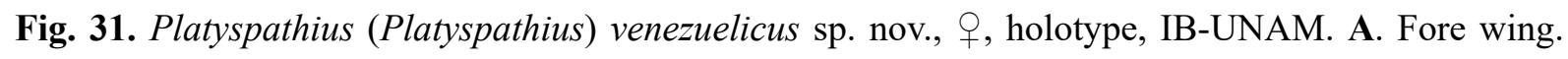
B. Hind wing, C. Metasoma, dorsal view. D. Metasoma, lateral view. 
long as weakly convex scutellum. Scutellum 0.9 times as long as its maximum apical width. Subalar depression shallow, rather wide, entirely coriaceous, without rugosity. Sternaulus (precoxal furrow) very shallow, straight, finely coriaceous and without crenulation, running along anterior 0.6 of lower part of mesopleuron. Metanotal tooth absent. Metapleural antero-lower lobe wide, short, subpointed apically.

WINGS. Fore wing 3.8-4.0 times as long as its maximum width. Radial vein (r) arising weakly before middle of pterostigma, from its 0.45 . Radial (marginal) cell not shortened. Metacarpus (1-R1) 1.1-1.2 times as long as pterostigma. Second radial abscissa (3-SR) 3.3-3.8 times as long as first abscissa (r) and forming with it weakly obtuse angle, $0.70-0.75$ times as long as third abscissa (SR1), 1.41.6 times as long as first radiomedial vein (2-SR). First medial abscissa $(1-\mathrm{SR}+\mathrm{M})$ sinuate. Second radiomedial (submarginal) cell not widened distally, its length 3.0-3.4 times maximum width, 1.351.45 times length of the narrow brachial (subdiscal) cell. Nervulus (cu-a) weakly declivous. Distance from nervulus (cu-a) to basal (1-M) vein about 0.2 times nervulus (cu-a) length. Parallel vein (CU1a) interstitial. Mediocubital vein (M+CU1) submedially strongly curved towards anal vein (2-1A). Brachial (subdiscal) cell narrowed towards apex, apically gently closed on or weakly before level of recurrent vein (m-cu). Hind wing 5.0-6.0 times as long as wide. First abscissa of costal vein $(\mathrm{C}+\mathrm{SC}+\mathrm{R}) 0.55-0.60$ times as long as second abscissa (1-SC+R). First abscissa of mediocubital vein $(\mathrm{M}+\mathrm{CU}) 0.4$ times as long as second and third abscissae combined (1-M). Recurrent vein (m-cu) strongly declivous towards base of wing, antefurcal, sclerotised.

LEGS. Hind coxa with distinct basoventral corner and very small tubercle. Hind femur 3.4-3.5 times as long as wide. Hind tibia with five apical spines on its outside apical margin. Hind tarsus as long as hind tibia. Hind basitarsus 0.7 times as long as second-fifth segments combined. Second segment of hind tarsus 0.5 times as long as basitarsus, 1.2-1.3 times as long as fifth segment (without pretarsus).

Metasoma. Length 1.1-1.3 times as long as head and mesosoma combined. Petiole dorsally distinctly convex in basal $0.20-0.25$, then almost straight (lateral view), more or less evenly and linearly widened from base to apex (dorsal view), with small spiracular tubercles in basal 0.3 of petiole. Acrosternite of first segment rather short, $0.30-0.35$ times as long as petiole tergite. Length of petiole 1.9-2.3 times its apical width, 1.5-1.7 times length of propodeum; maximum apical width of petiole about twice its minimum width. Second tergite without furrows. Median length of second tergite 0.8-0.9 times its basal width, 1.5-1.7 times length of third tergite. Second suture shallow, rather narrow, entirely finely crenulate with reticulation. Ovipositor sheath $0.7-0.8$ times as long as metasoma, 2.3-2.5 times as long as petiole, $1.2-1.3$ times as long as mesosoma, $0.60-0.65$ times as long as fore wing.

SCULPTURE AND PUBESCENCE. Vertex finely and very densely aciculate-coriaceous, sculpture arranged in transverse curved and dense aciculation usually, sometimes partly with reticulation; frons reticulatecoriaceous, rugulose-aciculate medially. Temple entirely coriaceous, fine below. Face densely granulate with reticulation. Mesoscutum densely and finely reticulate-coriaceous, parts of lobes near notauli without rugae, with single or double fine narrow median carina in medioposterior third of mesoscutum. Scutellum densely and finely coriaceous. Mesopleuron very finely reticulate-coriaceous, distinctly coriaceous below sternaulus. Metapleuron finely reticulate-coriaceous, rugose posteriorly at short area. Propodeum densely and distinctly reticulate-rugulose, more coarsely in posterior half, without delineated areas, but with two long lateral and single median carinae in basal $0.7-0.8$ of propodeum. Hind coxae coriaceous. Hind femur coriaceous distinctly dorsally to very weak ventrally. Petiole and second tergite very densely and distinctly reticulate-areolate, areolation very small and regular, second tergite basally without any striae. Second suture distinctly shortly crenulate with fine granulation between striae. Third tergite finely and densely reticulate-coriaceous, very weakly coriaceous posteriorly. Fourth to sixth tergites entirely very weakly areolate-coriaceous. Vertex mainly glabrous. Mesoscutum mainly glabrous, with sparse, short and semi-erect pale setae arranged narrowly along notauli and marginally. 
Hind tibia dorsally with sparse and rather long semi-erect white setae; length of these setae $0.5-0.8$ times maximum width of hind tibia.

Colour. Body dark reddish brown, head almost entirely, mesosoma and metasoma in anterior halves reddish brown or at least paler than rest body. Antenna yellow or yellowish brown in basal third or half, darkened towards apex, dark brown or black in apical part. Palps dark brown. Legs reddish brown, partly dark reddish brown, middle coxa light reddish brown, middle and hind tibiae almost black, all tarsi entirely brownish yellow with dark apical segment; all tibiae without pale subbasal rings. Ovipositor sheath yellow or pale brown in basal third, black on rest part. Fore wing distinctly and rather strongly maculate, with rather narrow hyaline stripes: basally, on level of maximum narrowed submedial (subbasal) cell, under base of pterostigma (interrupted medially), and widely apically; with small subhyaline spots in discoidal (discal) and radial (marginal) cells. Pterostigma mostly dark brown, whitish yellow in its basal 0.3.

\section{Male}

Unknown.

\section{Diagnosis}

We could not distinguish external morphological differences between this new species and the very similar Old World P. ornatulus (Enderlein, 1912) (Figs 32-33). However, the uncorrected COI distances between them are considerably high $(9.4 \%)$, and therefore we have decided to recognize them as separate species. The latter species occurs in the Oriental and Palaearctic regions, whereas $P$. venezuelicus sp. nov. represents the first species of the genus that has been recored for the American continent.

\section{Distribution}

Venezuela.

Platyspathius (Platyspathius) ranomafanus sp. nov. urn:lsid:zoobank.org:act:9FD75B8F-32F5-443D-A431-A7F884FEE605

Figs $34-35$

\section{Etymology}

Named from Ranomafana National Park in Madagascar, where type material was collected.

\section{Material examined}

\section{Holotype}

MADAGASCAR - $\odot$; "Madagascar, Fianarantsoa, Parc Nat. Ranomafana, radio tower forest edge, 7-17/VII/2003, 1130 m. Calif. Acad. Sci. Col. R, Harinhala. Malaise, mixed tropical forest, Caslot 014026, $21^{\circ} 15.05^{\prime} \mathrm{S} 47^{\circ} 24.43^{\prime} \mathrm{E}, \mathrm{MA}-02-09 \mathrm{~B}-66$. CNIN1443”; ZISP.

\section{Paratype}

MADAGASCAR・1 q; "Madagascar, Fianarantsoa, Parc Nat. Ranomafana, radio tower@ forest edge, 15-27/IV/2003, 1130 m. Calif. Acad. Sci. Col., Malaise, mixed tropical forest, R, Harin Hala., Caslot 014026, $21^{\circ} 15.05^{\prime} \mathrm{S} 47^{\circ} 24.43^{\prime} \mathrm{E}, \mathrm{MA}-02-09 \mathrm{~B}-58$. CNIN1351"; without right antenna, fore- and hind wings; IB-UNAM.

\section{Description}

\section{Female}

MeASUREMENTS. Body length 4.0-4.1 mm; fore wing length 2.9-3.0 mm. 


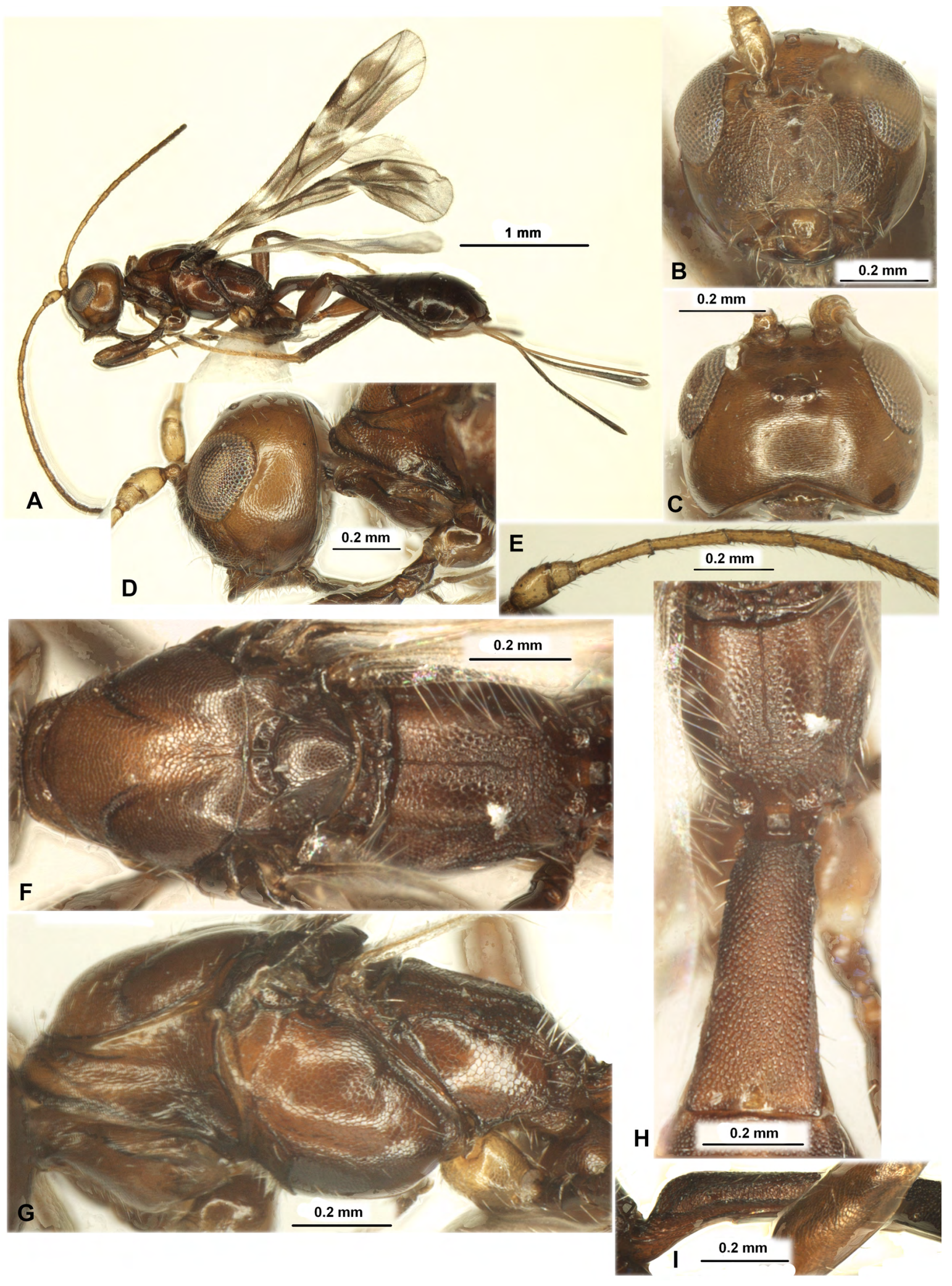

Fig. 32. Platyspathius (Platyspathius) ornatulus Enderlein, 1912, $q$, lectotype, MIZW. A. Habitus, lateral view. B. Head, lateral view. C. Head, front view. D. Head, dorsal view. E. Apical segments of antenna. F. Basal segments of antenna. G. Mesosoma, lateral view. H. Mesosoma, dorsal view. I. Hind femur and tibia, lateral view. J. First metasomal tergite, lateral view. 

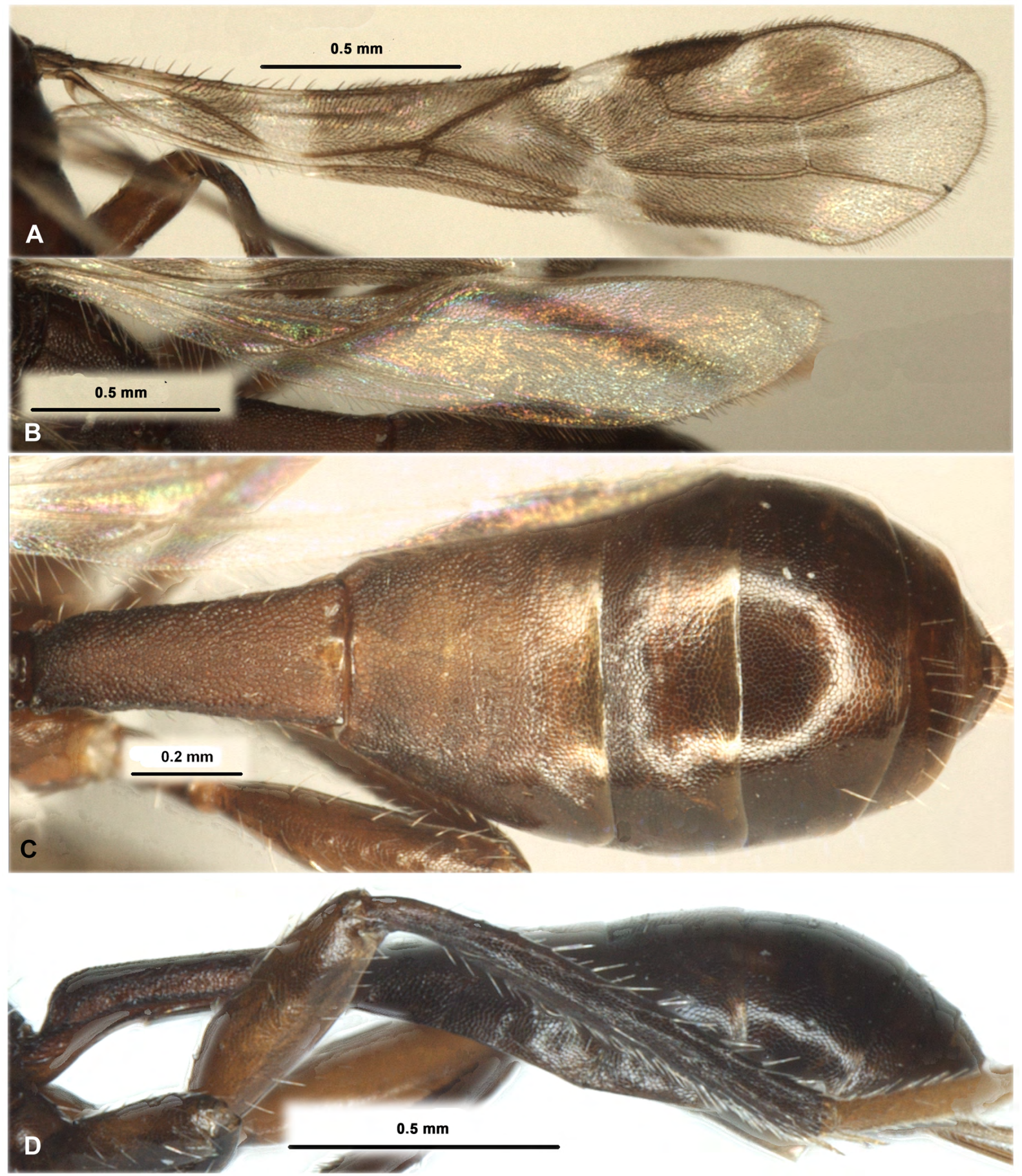

Fig. 33. Platyspathius (Platyspathius) ornatulus Enderlein, 1912, $q$, lectotype, MIZW. A. Fore wing. B. Hind wing, C. Metasoma, dorsal view. D. Metasoma, lateral view. 


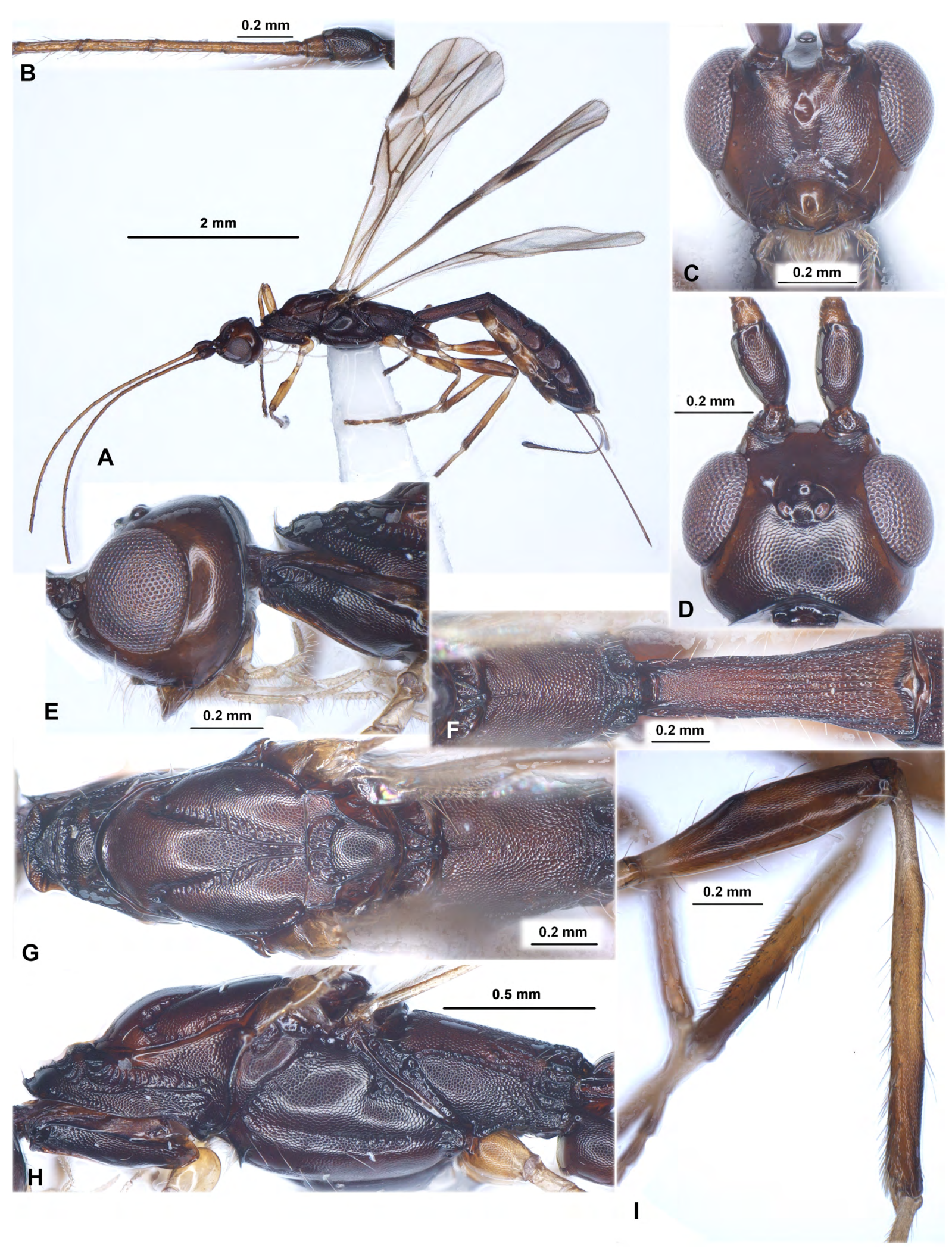

Fig. 34. Platyspathius (Platyspathius) ranomafanus sp. nov., ${ }^{+}$, holotype, ZISP. A. Habitus, lateral view. B. Basal segments of antenna. C. Head, front view. D. Head, dorsal view. E. Head, lateral view. F. Propodeum and first metasomal tergite, dorsal view. G. Mesosoma, dorsal view. H. Mesosoma, lateral view. I. Hind femur and tibia, lateral view. 


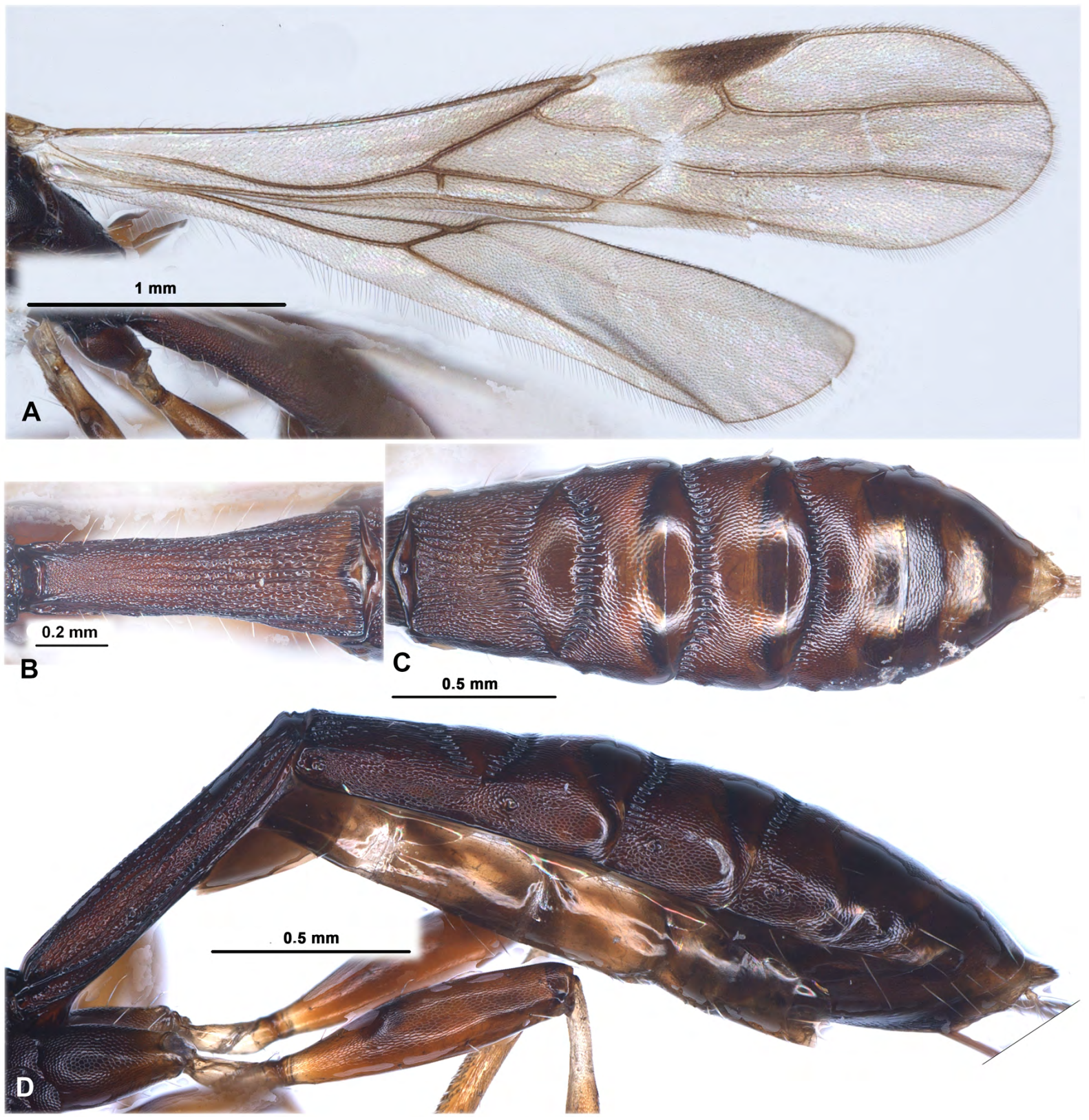

Fig. 35. Platyspathius (Platyspathius) ranomafanus sp. nov. $q$, holotype, ZISP. A. Wings. B. First metasomal tergite, dorsal view., C. Metasoma without first tergite, dorsal view. D. Metasoma, lateral view. 
HeAD. Width (dorsal view) 1.3-1.4 times median length, 1.2-1.3 times maximum width of mesoscutum. Head behind eyes (dorsal view) distinctly, evenly and weakly roundly narrowed; transverse diameter of eye 1.9-2.1 times as long as temple. Ocelli medium-sized, arranged in triangle with base 1.1-1.2 times its sides. POL 1.5-2.0 times OD, 0.7-0.8 times OOL. Frons medially with shallow longitudinal furrow. Eye glabrous, 1.2 times as high as broad. Malar space 0.4 times height of eye, almost equal to basal width of mandible. Face width 1.05-1.10 times height of eye and 1.15-1.20 times height of face and clypeus combined. Clypeal suture distinct laterally, but fine medially, without additional carina. Hypoclypeal depression medium-sized and round, its width 0.7-0.8 times distance from edge of depression to eye, 0.4 times width of face. Occipital carina complete dorsally, ventrally not joined with hypostomal carina being obliterated at short distance before base of mandible. Postgenal bridge narrow. Head below eyes (front view) distinctly and weakly-roundly narrowed. Antennae slender, weakly setiform, rather sparsely and rather long setose, 32-segmented, 1.3 times as long as body. Scapus thickened, 1.6-1.7 times as long as its maximum width. First flagellar segment 5.76.0 times as long as apical width, almost as long as or weakly shorter than second segment. Penultimate segment 4.0 times as long as its width, 0.6 times as long as first segment, 0.9 times as long as apical segment; the latter acuminate apically and without spine.

Mesosoma. Mesosoma distinctly elongate, weakly depressed dorso-ventrally, its length 2.7-2.8 times its height. Pronotal keel distinct, situated near middle of convex dorsal side of pronotum. Pronotal lateral oblique depressions rather wide, rather deep, densely and distinctly or finely crenulate, below in posterior third with rather distinct longitudinal stria. Mesoscutum (lateral view) weakly, uniformly and gently-roundly elevated above pronotum. Notauli wide, distinct, complete, entirely crenulate. Prescutellar depression long, shallow, weakly curved, with distinct median carina, entirely densely and weakly rugulose-reticulate, 0.3 times as long as weakly convex scutellum. Scutellum about as long as its maximum apical width. Subalar depression distinct, narrow, entirely crenulate with dense granulation. Sternaulus (precoxal furrow) deep, narrow, weakly sinuate, coriaceous and with narrow crenulation, running along whole length of lower part of mesopleuron. Metanotal tooth present, rather short, narrow and distinct acuminated apically. Metapleural antero-lower lobe wide, short, rounded apically.

Wings. Fore wing 4.2-4.8 times as long as its maximum width. Radial vein (r) arising weakly behind middle of pterostigma (from its 0.55 ) or submedially. Radial (marginal) cell not shortened. Metacarpus (1-R1) about as long as pterostigma. Second radial abscissa (3-SR) 5.0-5.5 times as long as first abscissa (r) and forming with it weakly obtuse angle, $0.8-0.9$ times as long as the straight third abscissa (SR1), 1.9-2.0 times as long as first radiomedial vein (2-SR). First medial abscissa (1-SR+M) distinctly sinuate. Second radiomedial (submarginal) cell weakly widened distally, its length 3.2-3.7 times maximum width, 1.3-1.7 times length of relatively narrow brachial (subdiscal) cell. Nervulus (cu-a) weakly declivous or perpendicular. Distance from nervulus (cu-a) to basal (1-M) vein 0.7-0.8 times nervulus (cu-a) length. Parallel vein (CU1a) interstitial. Mediocubital vein (M+CU1) submedially distinctly curved towards anal vein (2-1A). Brachial (subdiscal) cell weakly narrowed towards apex, apically gently closed weakly before level of recurrent vein (m-cu). Hind wing 6.0-6.3 times as long as wide. First abscissa of costal vein $(\mathrm{C}+\mathrm{SC}+\mathrm{R})$ 0.35-0.40 times as long as second abscissa (1-SC+R). First abscissa of mediocubital vein $(\mathrm{M}+\mathrm{CU})$ 0.3-0.4 times as long as second abscissa (1-M). Recurrent vein (m-cu) completely absent.

LEGS. Hind coxa with distinct basoventral corner and wide and small tubercle. Hind femur long, 4.0-4.5 times as long as maximum width. Hind tibia without apical spines on its outside apical margin. Hind tarsus as long as hind tibia. Hind basitarsus 0.7 times as long as second-fifth segments combined. Second segment of hind tarsus 0.45 times as long as basitarsus, 1.2-1.4 times as long as fifth segment (without pretarsus). 
Metasoma. Length 1.2 times as long as head and mesosoma combined. Petiole mostly straight dorsally entirely very weakly convex (lateral view), evenly and almost linearly widened from base to apex (dorsal view), without distinct spiracular tubercles. Acrosternite of first segment long, $0.45-0.50$ times as long as tergite. Length of petiole 2.4-2.5 times its apical width, 1.5-1.6 times length of propodeum; maximum apical width of petiole 2.0-2.3 times its minimum width. Second tergite elongate, with distinct convex crenulate furrow in posterior third, delineated wide lenticular apical area, its median length $0.7-0.8$ times remaining part of tergite. Median length of second tergite 1.1-1.2 times its basal width, 1.8-2.3 times length of third tergite. Second suture sinuate, deep, wide, entirely distinctly crenulate. Fifth and sixth tergite weakly enlarged, their posterior margins weakly convex or almost straight; fifth tergite almost equal to fourth and sixth tergites. Ovipositor sheath 0.6-0.7 times as long as metasoma, 1.9-2.0 times as long as petiole, almost equal to mesosoma, $0.45-0.50$ times as long as fore wing.

SCULPTURE AND PUBESCENCE. Vertex entirely finely coriaceous; frons very finely coriaceous to almost smooth partly; face granulate-coriaceous, smooth medially; temple distinctly coriaceous, becoming fine below. Mesoscutum densely and distinctly small reticulate-coriaceous, parts of lobes near notauli without rugae, with double distinct and convergent posteriorly carinae in medioposterior quarter of mesoscutum. Scutellum densely and finely granulate. Mesopleuron entirely densely and small reticulatecoriaceous. Metapleuron distinctly reticulate-granulate in anterior half, granulate-rugose in posterior half. Propodeum mostly and very densely areolate-granulate, coarsely rugose with granulation in posterior quarter, without delineated areas, but with short median longitudinal carina in basal $0.20-0.25$ of propodeum. Hind coxae reticulate-coriaceous; hind femur finely reticulate-coriaceous, almost smooth ventrally. Petiole striate with very dense reticulation between striae. Second tergite curvedly striate in basal half, mostly very densely reticulate-rugulose, apical area finely or very finely coriaceous. Second suture distinctly crenulate and sometimes with fine granulation between striae. Third-fifth tergites finely and densely reticulate-coriaceous becoming finely to distal tergites, their posterior margins very weakly coriaceous to almost smooth. Vertex almost entirely glabrous. Mesoscutum mainly glabrous, with very sparse, short and semi-erect pale setae arranged narrowly along posterior half of notauli and marginally. Hind tibia dorsally with very sparse, long and partly short semi-erect yellowish setae; length of these setae $0.7-1.2$ times maximum width of hind tibia.

CoLour. Body mainly dark reddish brown, metasoma sometimes mostly reddish brown. Antenna light reddish brown or reddish brown, infuscate towards apex, scapus sometimes dark reddish brown. Palps yellow. Fore and middle legs light reddish brown to yellowish brown with several brown sports; hind leg reddish brown to dark reddish brown (especially coxa), trochanter and base of tibia yellow. Ovipositor sheath mostly brown, pale brown basally. Fore wing distinctly and rather evenly infuscate, brownish, without hyaline stripes. Pterostigma mostly dark brown, whitish yellow in basal 0.3 .

\section{Male \\ Unknown.}

\section{Diagnosis}

This new species has a distinct, complete and crenulate notauli and delineated apical lenticular area on second metasomal tergite. By these features it is similar to the Korean P. hospitus Belokobylskij \& $\mathrm{Ku}, 2001$, but distinctly differs from it by having the meso- and metasoma long and slender (vs short and stocky), head subround dorsally (vs transverse), mesoscutum weakly elevated upper pronotum (vs distinctly elevated), and petiole and its acrosternite long (vs short).

\section{Distribution}

Madagascar. 
BELOKOBYLSKIJ S.A. \& ZALDÍVAR-RIVERÓN A., Reclassification of the Rhaconotini

Subgenus Lenticularia van Achterberg, 2003

Platyspathius (Lenticularia) van Achterberg, 2003: 283.

Platyspathius (Lenticularia) - Yu et al. 2016.

\section{Type species}

Doryctes lenticularis Granger, 1949, by monotypy and original designation (van Achterberg 2003).

Description (Figs 36-37)

HEAD. Head weakly depressed and weakly transverse. Vertex entirely densely and distinctly transverse striate. Ocelli arranged in almost equilateral triangle. Eyes glabrous. Occipital carina dorsally complete, obliterate below and not joining ventrally with hypostomal carina. Malar suture rather distinct and complete. Hypoclypeal depression medium size, round. Postgenal bridge narrow. Scapus wide and long. First flagellar segment subcylindrical, almost straight, longer than second segment.

Mesosoma. Mesosoma weakly depressed and long. Neck of prothorax long; pronotum dorsally distinctly convex with submedian shallow cavity, with rather distinct carina situated in anterior 0.3 of pronotum. Mesonotum highly and perpendicularly and later roundly elevated above pronotum, densely and distinctly granulate. Median lobe of mesonotum highly hump-shaped (lateral view), without median longitudinal furrow, without anterolateral corners. Notauli incomplete, deep anteriorly and shallow posteriorly. Prescutellar depression long, with distinct median carina. Scutellum distinctly convex, with distinct lateral carinae. Metanotum without tooth (lateral view). Sternaulus (precoxal sulcus) distinct, running along entire lower length of mesopleuron. Propodeum without areas and carinae; lateral tubercles absent.

WINGs. Wings not shortened. Pterostigma of fore wing rather narrow and long. Radial vein (r) arising from middle of pterostigma. Radial (marginal) cell not shortened. Both radiomedial veins (2-SR, r-m) present. Second radiomedial (submarginal) cell very long, wide, distinctly widened towards apex. Recurrent vein (m-cu) postfurcal. Recurrent (m-cu) and basal (1-M) veins distinctly divergent posteriorly. Nervulus (cu-a) postfurcal, subvertical. Brachial (subdiscal) cell wide, narrowed apically, gently curvedly closed postero-apically on level of recurrent vein (m-cu). Parallel vein (CU1a) almost interstitial. In hind wing, first abscissa of mediocubital vein $(\mathrm{M}+\mathrm{CU}) 0.6$ times as long as second abscissa (1-M).

LEGS. Middle tarsal segments shortened. Hind coxa long and narrow, with distinct basoventral corner and distinct tubercle. Hind femur rather narrow, elongate-oval. Hind tibia thickened. Hind basitarsus 0.75 times as long as second-fifth segments combined.

Metasoma. First tergite semi-petiolate, long, rather narrow, but distinctly widened towards apex; acrosternite of first segment about 0.25 times as long as first tergite. Second tergite without basal and apical area and without furrows. Second suture very shallow, narrow, complete and weakly sinuate. Third tergite without transverse depression or furrow. Present all visible dorsally tergites, fifth and sixth tergites short. Setae on fourth to sixth tergites rather widely distributed laterally and absent medially. Ovipositor sheath about as long as metasoma.

\section{Diagnosis}

The subgenus Lenticularia differs from Platyspathius s. str. by the following characters: 1) vertex entirely densely and distinctly transverse striate; 2) malar suture rather distinct and complete; 3 ) median lobe of mesonotum highly hump-shaped; 4) second radiomedial (submarginal) cell considerably long, wide, and distinctly widened towards apex. 


\section{Composition}

Platyspathius (Lenticularia) lenticularis (Granger, 1949).

\section{Distribution}

Afrotropical region.

Platyspathius (Lenticularia) lenticularis (Granger, 1949)

Figs 36-37

Doryctes lenticularis Granger, 1949: 122.

Platyspathius lenticularis - Shenefelt \& Marsh 1976: 1385.

Platyspathius (Lenticularia) lenticularis - van Achterberg 2003: 283. — Yu et al. 2016.

\section{Material examined}

\section{Holotype}

MADAGASCAR • ' ; "Madagascar, Tsinjoarivo, 21.II.32., A. Seyrig”, "Type", "Doryctes lenticularis Gr., det P. Marsh", "Holotypus Doryctes lenticularis Gr., des. Marsh 1976", "Platyspathius lenticularis (Grang.), det. Belokobylskij '94”; MNHN.

\section{Description}

\section{Female}

Measurements. Body length $5.9 \mathrm{~mm}$; fore wing length $4.5 \mathrm{~mm}$.

HEAD. Width 1.25 times its median length, 1.2 times width of mesoscutum. Head behind eyes (dorsal view) weakly roundly narrowed. Transverse diameter of eye 1.7 times length of temple. Ocelli rather small, arranged in triangle with base almost equal to its sides; POL 1.4 times OD, 0.6 times OOL. Eye 1.2 times as high as broad. Malar space 0.6 times height of eye, 1.15 times basal width of mandible. Face distinctly convex, its width 1.2 times height of eye and 1.6 times height of face and clypeus combined. Clypeus low, its upper margin situated distinctly below than lower level of eyes. Hypoclypeal depression round, its width 0.65 times distance from edge of depression to eye, 0.4 times width of face. Vertex weakly convex. Head below eyes distinctly and weakly-roundly narrowed. Antennae rather slender, almost filiform, more than 7-segmented (most part of segments missing). Scapus 2.0 times as long as its maximum width. First flagellar segment 6.5 times as long as its apical width, 1.25 times as long as second segment.

Mesosoma. Length 2.5 times its height. Mesoscutum (dorsal view) as long as maximum width, its median lobe distinctly convex anteriorly. Notauli complete, entirely rugose-crenulate. Prescutellar depression rather shallow, long, distinctly and almost linearly oblique posterolaterally, entirely and rather densely reticulate-rugose with short striae, 0.4 times as long as scutellum. Sternaulus (precoxal furrow) weakly curved, almost entirely smooth. Prepectal carina belowe with weakly widened ventral lobes opposite fore coxae. Subalar depression coarsely longitudinally striate with granulation marginally.

WiNGs. Fore wing 4.4 times its maximum width. Radial (marginal) cell 4.5 times as long as wide. Metacarpus (1-R1) 1.3 times as long as pterostigma. First radial abscissa (r) forming right angle with second abscissa (3-SR). Second radial abscissa (3-SR) weakly curved, 5.4 times as long as first abscissa (r), 1.8 times as long as almost straight third abscissa (SR1), 2.5 times as long as first radiomedial vein (2-SR). Second radiomedial (submarginal) cell long, weakly widened distally, 3.3 times as long as wide, 1.2 times as long as the narrow brachial (subdiscal) cell. First medial abscissa (1-SR+M) strongly sinuate. Recurrent vein (m-cu) 2.3 times as long as second median abscissa (2-SR+M), 0.6 times as 


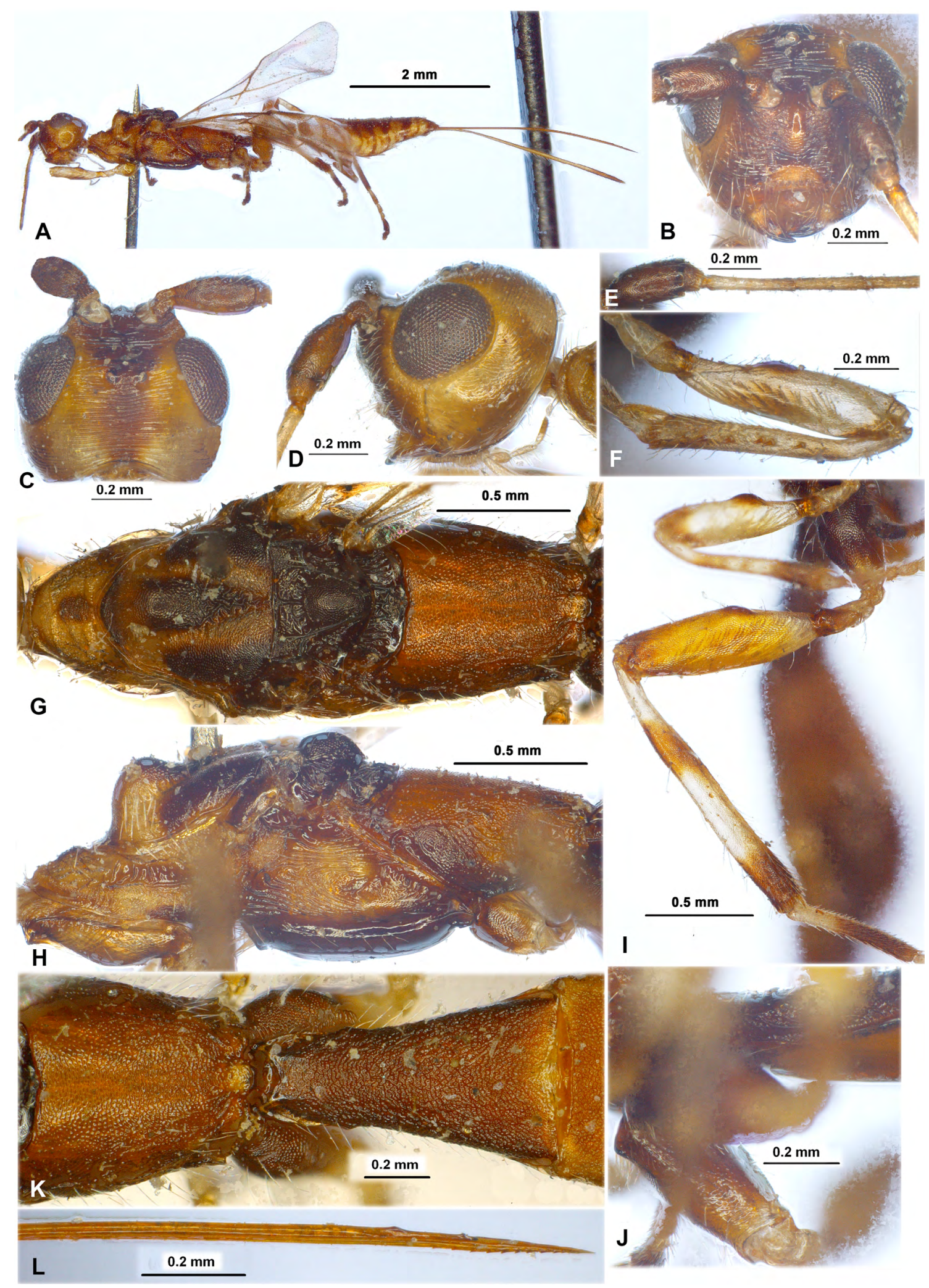

Fig. 36. Platyspathius (Lenticularia) lenticularis (Granger, 1949), ㅇ, holotype, MNHN. A. Habitus, lateral view. B. Head, front view. C. Head, dorsal view. D. Head, lateral view. E. Basal segments of antenna. F. Fore leg. G. Mesosoma, dorsal view. H. Mesosoma, lateral view. I. Hind leg. J. Hind coxa. K. Propodeum and first metasomal tergite, dorsal view. L. Apex of ovipositor, lateral view. 


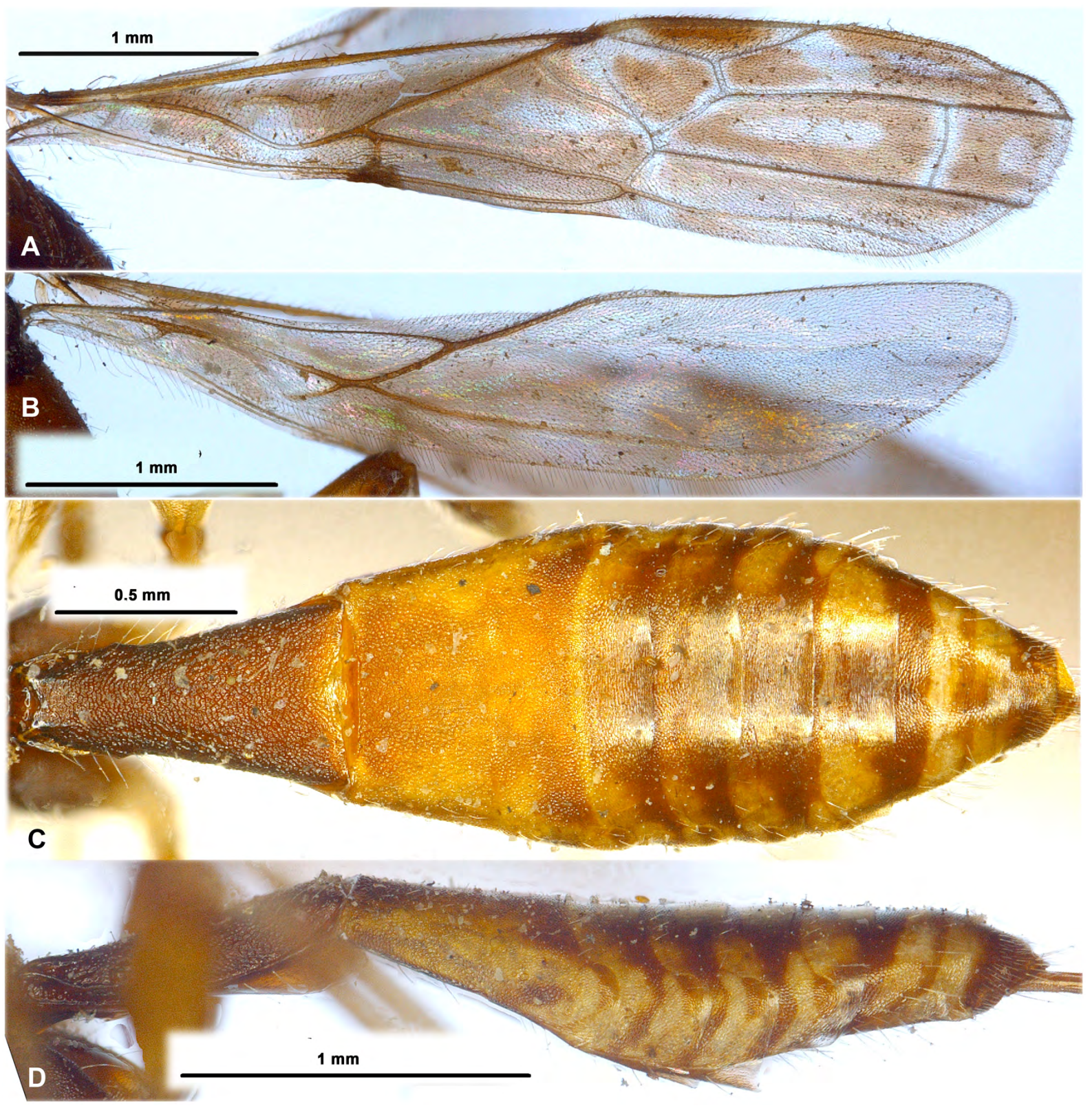

Fig. 37. Platyspathius (Lenticularia) lenticularis (Granger, 1949),, , holotype, MNHN. A. Fore wing. B. Hind wing, C. Metasoma, dorsal view. D. Metasoma, lateral view. 
long as first radiomedial vein (2-SR). Mediocubital vein (M+CU1) strongly curved to anal vein (2-1A) in distal third. Distance between nervulus (cu-a) and basal (1-M) vein 0.4 times nervulus (cu-a) length. Brachial (subdiscal) cell closed shortly behind level of recurrent vein; posterior bulla and posterior abscissa of anal vein (2-1A) (behind brachial vein (CU1b)) absent. Hind wing about 5.0 times as long as wide. Recurrent vein (m-cu) more or less sclerotised, pigmented.

LeGs. Fore femur about 4.0 times as long as wide. Hind coxa 1.5 times as long as wide, 0.75 times as long as propodeum. Hind femur 3.5 times as long as maximum width. Hind tibia with dense comb of rather long pale setae on inner apical margin. Hind tarsus as long as hind tibia. Second tarsal segment 0.4 times as long as basitarsus, 2.7 times as long as fourth segment, as long as fifth segment (without pretarsus).

Metasoma. Length about as long as head and mesosoma combined. First tergite almost entirely flat (lateral view), distinctly and linearly widened towards apex (dorsal view); maximum width of tergite 2.0 times its basal width; its length 1.75 times apical width, 1.35 times length of propodeum. Median length of second tergite 0.65 times its basal width, 0.9 times length of third tergite. Second suture very shallow, narrow, weakly evenly curved. Fifth and sixth tergite short, straight on posterior margins, without any median emargination and postero-ventral lobes. Ovipositor sheath very weakly widened towards subapex, then distinctly narrowed towards apex; about as long as metasoma, 1.4 times as long as mesosoma, 0.7 times as long as fore wing.

SCULPTURE AND PUBESCENCE. Vertex and frons entirely distinctly, densely and weakly sinuate striate; face densely and distinctly curvedly striate with rugulosity between striae in upper half; temple distinctly curvedly striate in upper half, sculpture becoming fine below and almost smooth in lower part. Mesoscutum and scutellum entirely densely granulate, mesoscutum with narrow rugose subtriangular area in its medioposterior half. Mesopleuron mostly longitudinally and partly curvedly striate, with reticulation postero-ventrally, almost smooth below sternaulus. Metapleuron distinctly and small rugosereticulate with granulation, partly with curved longitudinal striae. Propodeum very densely and small reticulate-areolate, posteriorly with additional rugosity. Hind coxa almost entirely densely and distinctly granulate. Hind femur entirely densely and distinctly reticulate-granulate becoming coriaceous in lower part. First, second and most part of third tergites entirely, very densely and small or very small reticulateareolate, without any striation, sculpture becoming finer to end of third tergite. Fourth to sixth tergites very densely and finely or very finely transverse aciculate and coriaceous. Vertex glabrous in anterior half, in sparse, short and semi-erect pale setae in posterior half. Mesoscutum mainly glabrous, with sparse and short semi-erect pale setae in anterolateral corners, along notauli and marginally. Mesopleuron widely glabrous. Hind tibia dorsally with rather long, sparse, semi-erect pale setae; length of these setae 0.6-0.8 times maximum width of hind tibia.

CoLOuR. Head yellow to yellowish brown, with brown longitudinal and rather wide stripe on middle on vertex and frons, face mainly brown. Mesosoma mainly brownish yellow or yellow, mesonotum, lower part of meso- and metapleuron dark reddish brown to almost black. Metasoma reddish brown, second and most part of third tergites as well as sides of rest tergites yellow. Scapus and pedicel of antenna dark reddish brown, basal segments of antenna yellow to brownish yellow. Palps pale yellow. Legs mainly yellow or whitish yellow, middle and especially hind coxae, trochantelli partly, protuberances on femora, subbasal and apical wide stripes on hind tibia, most part of basitarsi and all fifth segments of tarsi brown to dark reddish brown; hind tibia pale yellow basally. Fore wing distinctly infuscate, with numerous small hyaline spots and stripes. Pterostigma brown, pale or whitish yellow in basal and apical 0.4 and along inner margin. 


\section{Male \\ Unknown. \\ Distribution}

Madagascar.

Genus Pseudorhaconotus van Achterberg \& Shaw, 2010

Pseudorhaconotus van Achterberg \& Shaw, 2010: 2.

Pseudorhaconotus - Yu et al. 2016. — Jasso-Martínez et al. 2019: 165.

\section{Type species}

Pseudorhaconotus enervatus van Achterberg \& Shaw, 2010, by monotypy and original designation (van Achterberg \& Shaw 2010).

\section{Description (Figs 38-39)}

HEAD. Head weakly transverse. Ocelli arranged in triangle with base larger than its sides. Frons flat and without median keel. Eyes with sparse and short setae. Occipital carina distinct dorsally, obliterated below at short distance and not fused with hypostomal carina. Malar suture absent. Clypeal suture distinct and complete. Clypeus with short and fine ventral flange. Hypoclypeal depression round. Postgenal bridge narrow. Palps relatively short; maxillary palps 6-segmented, labial palps 4-segmented; third segment of labial palps not shortened. Scapus rather wide and long, without apical lobe. First flagellar segment subcylindrical, almost straight, almost as long as second segment.

Mesosoma. Pronotum dorsally flat, pronotal keel distinct. Mesonotum not highly, gently roundly elevated above pronotum, entirely finely reticulate-granulate. Notauli entirely shallow and fine. Prescutellar depression rather short, with median carina. Scuto-scutellar suture present and distinct. Metanotal tooth almost absent. Sternaulus (precoxal furrow) shallow, long, sculptured. Prepectal carina distinct and complete. Postpectal carina absent. Propodeum with areas mostly delineated by distinct carinae in basal half and fine carinae in apical half, areola narrow; lateral tubercles absent; propodeal bridge absent; spiracles small and round.

LEGS. Fore and middle tibiae with slender several spines arranged in single row. Hind coxa with distinct basoventral corner and basoventral tooth. All femora with fine dorsal protuberances. Hind basitarsus almost 0.5 times as long as second-fifth segments combined.

WINGS. Pterostigma of fore wing narrow; radial vein (r) arising from middle of pterostigma. Radial (marginal) cell rather distinctly shortened. Second radiomedial vein ( $\mathrm{r}-\mathrm{m})$ absent. Recurrent vein (mcu) weakly postfurcal or almost interstitial. Nervulus (cu-a) postfurcal. Discoidal (discal) cell long petiolate. Parallel vein (CU1a) interstitial. Brachial (subdiscal) cell closed at level of recurrent vein (m-cu). Hind wing with three hamuli. Nervellus (cu-a) present. Medial (basal) cell narrow, but weakly widened posteriorly. Submedial (subbasal) cell rather short; first abscissa of mediocubital vein $(\mathrm{M}+\mathrm{CU})$ 0.6 times as long as second abscissa (1-M). Recurrent vein (m-cu) present, but transparent.

Metasoma. First metasomal tergite not petiolate, relatively long and wide, moveably fused with second tergite. Acrosternite $0.15-0.20$ times as long as first tergite, its apical margin situated distinctly before level of spiracles; dorsope of first tergite absent; spiracular tubercles small, dorsal carinae distinct, complete and strongly convergent basally; basolateral lobes absent. Second suture deep, almost straight, without lateral bends. Second tergite often with fine subbasal transverse furrow, with apical narrow area 
delineated anteriorly by deep and rather wide furrow. Present five visible tergites. Second-sixth tergites with separate laterotergites. Fourth and fifth tergite with distinct and crenulate subbasal transverse furrows. Fifth tergite of female enlarged, larger than previous tergite, almost concealed by the succeeding segments, entirely sculptured. Ovipositor much shorter than metasoma.

\section{Diagnosis}

This genus is morphologically similar to Rhaconotus Ruthe, 1854 but differs from the latter genus mainly by the missing second radiomedial vein $(\mathrm{r}-\mathrm{m})$ and second radiomedial (submarginal) cell of fore wing.

\section{Composition}

Pseudorhaconotus enervatus van Achterberg \& Shaw, 2010.

\section{Hosts}

Unknown.

\section{Distribution}

Palaearctic region.

\section{Remarks}

Pseudorhaconotus is a morphologically distinctive monotypic genus that was originally described from southern Spain (van Achterberg \& Shaw 2010). This is one of the two Rhaconotini genera (together with Malagasy Grangerdoryctes Belokobylskij, 2005) that does not have the second radiomedial vein (r-m) and second radiomedial (submarginal) cell. The position of this genus within Rhaconotini is supported by its enlarged fifth metasomal tergite, which almost covers the following segments, the separated laterotergites on the fifth metasomal tergite, interstitial position of parallel vein (CU1a) in fore wing, postfurcal position of recurrent vein (m-cu), and densely granulate-reticulate head and mesosoma.

\section{Pseudorhaconotus enervatus van Achterberg \& Shaw, 2010}

Figs 38-39

Pseudorhaconotus enervatus van Achterberg \& Shaw, 2010: 3.

Pseudorhaconotus enervatus - Yu et al. 2016. — Jasso-Martínez et al. 2019: 167.

\section{Material examined}

\section{Holotype}

SPAIN • O+; "Spain: Granada, Orgiva, 305 VF68, 500 m.", "Interception trap, 25.X.2007, Jose Luis Ruiz de la Cuesta", "Don. A. Ribes, 11223”, “O Pseudorhaconotus gen. nov. enervatus sp. nov., van Achterberg \& Shaw, 2010, Holotype"; NMS.

\section{Description}

\section{Female}

MEASUREMENTS. Body length $4.8 \mathrm{~mm}$; fore wing length $3.0 \mathrm{~mm}$.

HEAD. Width 1.4 times its median length, 1.3 times width of mesoscutum. Head behind eyes (dorsal view) distinctly and roundly narrowed; transverse diameter of eye 1.6 times length of temple. Ocelli small, arranged in triangle with base 1.25 times its sides; POL 2.0 times OD, 0.5 times OOL. Eye very weakly emarginated opposite antennal sockets, 1.2 times as high as broad. Malar space 0.55 times height of eye, equal to basal width of mandible. Face width 1.2 times height of eye and 1.4 times height of face and clypeus combined. Upper margin of clypeus situated distinctly below than lower 


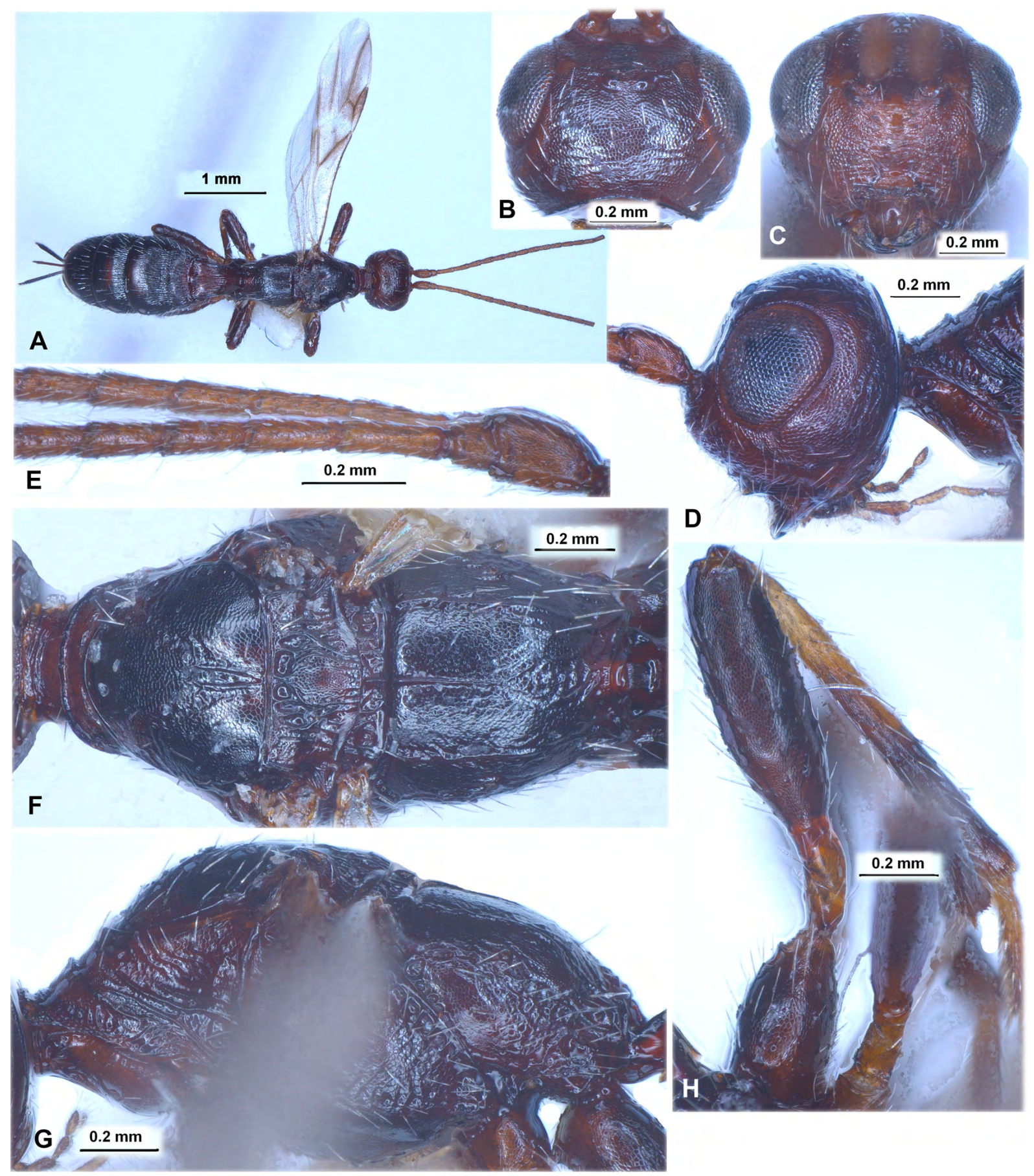

Fig. 38. Pseudorhaconotus enervatus van Achterberg \& Shaw, 2010, + , holotype, NMS. A. Habitus, dorsal view. B. Head, dorsal view. C. Head, front view. D. Head, lateral view. E. Basal segments of antenna. F. Mesosoma, dorsal view. G. Mesosoma, lateral view. H. Hind leg. 
BELOKOBYLSKIJ S.A. \& ZALDÍVAR-RIVERÓN A., Reclassification of the Rhaconotini
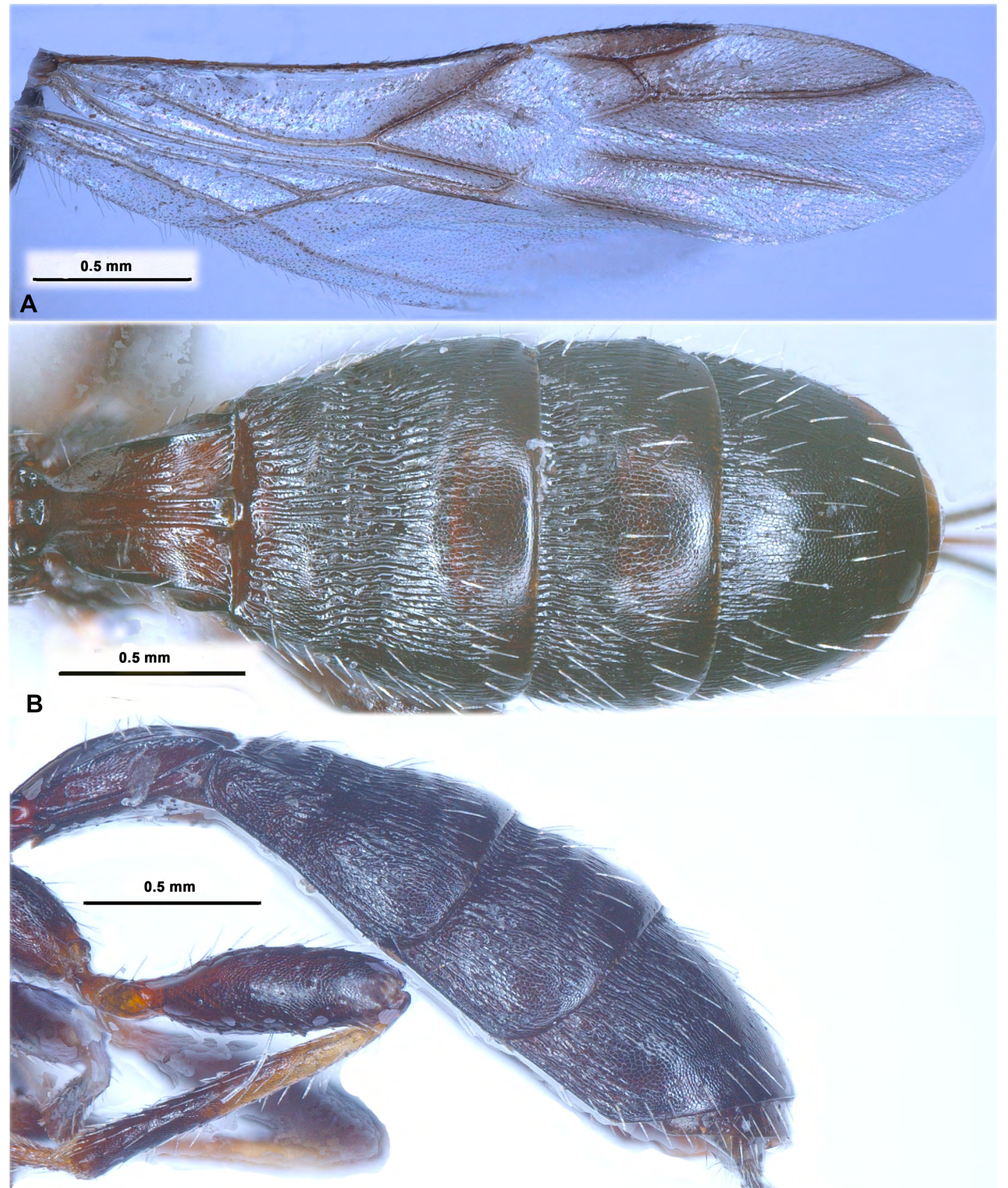

C

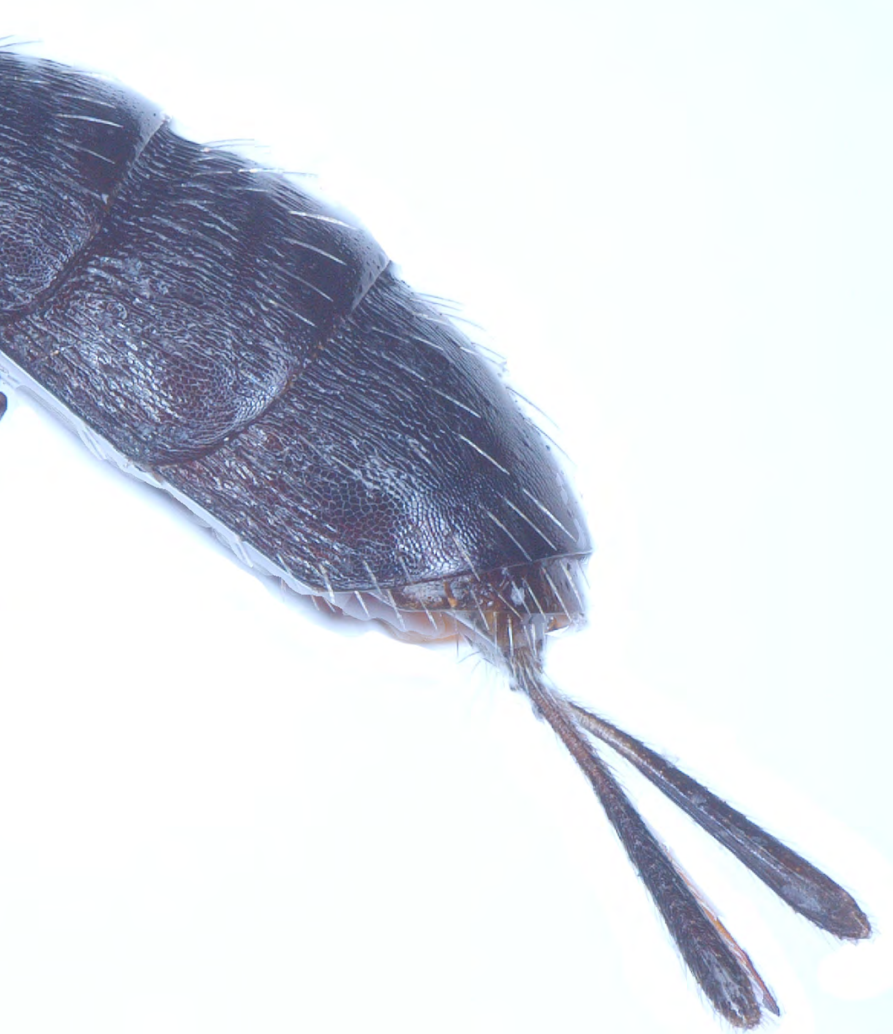

Fig. 39. Pseudorhaconotus enervatus van Achterberg \& Shaw, 2010, $q$, holotype, NMS. A. Wings. B. Metasoma, dorsal view. C. Metasoma and hind leg, lateral view. 
level of eyes. Hypoclypeal depression width 0.6 times distance from edge of depression to eye, 0.3 times width of face. Vertex distinctly convex. Head below eyes distinctly and almost linearly narrowed. Antennae rather slender, weakly setiform, about 32-segmented (accordingly description, in studied holotype kept only 15 segments). Scapus 1.8 times as long as its maximum width. First flagellar segment 3.3 times as long as its apical width, almost as long as second segment. Submedian segment 2.2 times as long as their width.

Mesosoma. Length 2.0 times its height. Distances from coarse pronotal keel to anterior margin of pronotum about 1.7 times larger than distance from keel to posterior margin of pronotum. Mesoscutum 0.9 times as long as its maximum width. Notauli complete, rather narrow, sparsely crenulate-rugulose with fine granulation. Median lobe of mesoscutum with distinct median furrow in its posterior 0.4. Prescutellar depression rather deep, distinctly and almost linearly widened towards lateral margins, with distinct five carinae, smooth or partly finely rugulose between carinae, medially 0.2 times as long as scutellum. Scutellum weakly convex, with lateral carinae, its maximum width 1.1 times median length. Sternaulus (precoxal furrow) shallow, wide, straight, distinctly crenulate with fine reticulation, running along anterior 0.7 of lower part of mesopleuron. Prepectal carina below with weakly widened lobes opposite fore coxae. Subalar depression rather deep, narrow, sparsely and coarsely crenulate with granulation. Mesopleural suture entirely, coarsely, sparsely and shortly crenulate. Metapleural lobe rather long, wide basally, rounded apically, without dense pubescence.

WINGS. Fore wing 4.3 times its maximum width, not coming forward apex of metasoma, apical margin of wing without setae. Radial (marginal) cell weakly shortened, narrow, 4.4 times as long as maximum width. Metacarpus (1-R1) 0.9 times as long as pterostigma, about 2.0 times as long as distance from apex of radial (marginal) cell to apex of wing. First radial abscissa (r) forming very obtuse angle with second abscissa (3-SR). Second radial abscissa (3-SR) very weakly curved, 6.2 times as long as first abscissa (r), 2.6 times as long as first radiomedial vein (2-SR). First medial abscissa (1-SR+M) weakly curved distally. Mediocubital vein (M+CU1) distinctly sinuate, distinctly curved to anal vein $(2-1 \mathrm{~A})$ in distal half. Discoidal (discal) cell narrow, 2.5 times as long as wide. Distance between nervulus (cu-a) and basal (1-M) vein 0.6 times nervulus (cu-a) length. Brachial (subdiscal) cell roundly closed almost on level of recurrent vein; posterior bulla and posterior abscissa of longitudinal anal vein (behind brachial vein (CU1b)) absent. Hind wing 4.6 times as long as wide. First costal abscissa (C+SC+R) 0.6 times as long as second abscissa (1-SC+R). First abscissa of mediocubital vein $(\mathrm{M}+\mathrm{CU}) 0.5$ times as long as second abscissa (1-M). Recurrent vein (m-cu) antefurcal.

LEGS. Hind coxa 1.5 times as long as wide (with tubercle). Hind femur 3.2 times as long as its maximum width. Hind tarsus 0.9 times as long as hind tibia. Second tarsal segment 0.6 times as long as basitarsus, 2.0 times as long as fourth segment, 1.3 times as long as fifth segment (without pretarsus).

Metasoma. Length 1.2 times as long as head and mesosoma combined. First tergite distinctly widened toward apex, with coarse lateral carinae Maximum width of first tergite 2.5 times its minimum width; its length 1.3 times apical width, almost equal to length of propodeum. Second tergite with very narrow basal area weakly delineated posteriorly by shallow furrow; with distinct, narrow, and straight transverse subposterior furrow weakly separated narrow subparallel apical area, median length of apical area 0.5 times length of rest part of tergite. Median length of second tergite 0.6 times its basal width, almost equal to length of third tergite. Second suture rather deep, wide, straight medially and weakly curved laterally. Fifth tergite strongly enlarged, weakly roundly convex in posterior margin, without median emargination, and postero-ventral lobes. Fifth tergite 1.3 times as long as fourth tergite, 1.5 times as long as third tergite. Ovipositor sheath 0.3 times as long as metasoma, 0.7 times as long as hind tibia, 0.5 times as long as mesosoma, 0.25 times as long as fore wing.

SCUlPTURE AND PUBESCENCE. Vertex rater finely and densely granulate, with more or less distinct and weakly undulate transverse striae; frons coarsely curvedly striate with fine granulation between 
striae partly, densely granulate and without striation laterally. Face densely and distinctly granulate, with striation on two narrow vertical submedian stripes, medially finely coriaceous to almost smooth; temple entirely granulate or granulate-coriaceous, additionally vertically coarsely striate in posterior 0.5-0.7, sculpture becoming finer towards its lower part. Mesoscutum entirely densely and rather finely granulate-reticulate, without rugosity along notauli, with two median striae in medioposterior 0.4 of mesoscutum and short rugosity between their and around. Scutellum densely and rather finely granulate-reticulate, laterally widely with distinct curved striation. Mesopleuron entirely densely and finely granulate-reticulate, below sternaulus finely reticulate-coriaceous. Metapleuron finely reticulatecoriaceous in anterior half, rugose-areolate in posterior half. Propodeum with long basolateral areas mainly finely coriaceous and with rugosity along carinae, with distinct and long median carina in basal 0.4 of propodeum; areola narrow, finely and irregularly delineated; propodeum rugose-striate in its posterior 0.3 . Hind coxa finely granulate-reticulate dorsally and reticulate-coriaceous laterally. Hind femur finely and densely reticulate-coriaceous becoming fine laterally, almost smooth below. First tergite densely and finely reticulate-coriaceous and medially with distinct and rather sparse wide striation. Second tergite almost entirely striate, with fine reticulation between striae and only reticulate on two medio-lateral narrow longitudinal stripes. Third tergite in its basal $0.5-0.7$, fourth on its basal $0.3-0.5$, and fifth basally striate, other parts of tergites densely granulate-reticulate becoming finely reticulatecoriaceous towards their posterior margins. Second-fifth tergites laterally entirely densely striate with fine reticulation between striae. Vertex with long, sparse, and almost adpressed pale setae. Mesoscutum with sparse, rather short and semi-erect setae arranged more or less widely along notauli and marginally, widely glabrous medially. Mesopleuron glabrous on narrow medial area. Hind tibia dorsally with rather long, sparse, and semi-erect pale setae; length of these setae 0.5-0.6 times maximum width of hind tibia.

CoLour. Head dark reddish brown, only reddish brown around eyes, anteriorly and below. Mesosoma reddish brown to dark reddish brown, dorsally almost black. Metasoma black with dark reddish brown spots, first tergite reddish brown. Antenna light reddish brown becoming darkened towards its middle. Palps yellowish brown, infuscate basally. Legs reddish brown to dark reddish brown, tarsi light reddish brown, fore and middle tibiae basally and hind tibia in its basal $0.3-0.4$ brownish yellow. Ovipositor sheath black, light brown basally. Fore wing hyaline, with two large darkened stripes: one stripe crossed basal (1-M) vein and brachial (subdiscal) cell, second stripe crossed wing area below its posterior 0.7 of pterostigma and on all radial (marginal) cell. Pterostigma dark brown, yellow in basal quarter.

\section{Male}

Unknown.

\section{Distribution}

Western Palaearctic (Spain).

Genus Rhaconotinus Hedqvist, 1965

Rhaconotinus Hedqvist, 1965: 8.

Rhaconotinus - Shenefelt \& Marsh 1976: 1330. — Belokobylskij 1992: 907 (as synonym of Rhaconotus); 1994a: 340; 2019: 36 (status resurrected). — Yu et al. 2016. — Belokobylskij et al. 2019: 267.

\section{Type species}

Rhaconotinus caboverdensis Hedqvist, 1965

\section{Description (Figs 40-43)}

HEAD. Head usually weakly transverse. Ocelli arranged in obtuse triangle with base larger than its sides. Frons flat and without median keel. Eyes glabrous. Occipital carina complete dorsally, obliterated below 
at short distance and not fused ventrally with hypostomal carina. Malar suture absent. Clypeal suture more or less distinct and usually complete. Clypeus with short ventral flange. Hypoclypeal depression medium size, subround. Postgenal bridge narrow. Palps relatively short; maxillary palps 6-segmented, labial palps 4-segmented; third segment of labial palps not shortened. Scapus rather wide, relatively short, without apical lobe and basal constriction. First flagellar segment subcylindrical, relatively slender, not shorter than second segment.

Mesosoma. Pronotum dorsally more or less distinctly convex, pronotal keel usually distinct. Mesonotum weakly but distinctly and often gently-roundly elevated above pronotum, usually mostly entirely granulate. Notauli deep and complete, sometimes distinctly shallow posteriorly. Scuto-scutellar suture distinct. Prescutellar depression relatively long or short, always with median carina. Sternaulus (precoxal furrow) rather shallow, long, curved. Prepectal carina distinct and complete. Postpectal carina absent. Metanotum with short median subrounded or subpointed tooth. Propodeum with basolateral areas delineated by fine or distinct carinae, without areola; lateral tubercles absent or small; propodeal bridge absent; spiracles small and round.

Wings. Pterostigma of fore wing long and rather wide. Radial vein (r) arising mostly from middle of pterostigma. Radial (marginal) cell long, never shortened. Both radiomedial veins (2-SR, r-m) present. Recurrent vein (m-cu) postfurcal, sometimes only weakly. Discoidal (discal) cell distinctly petiolate. Nervulus (cu-a) always postfurcal. Brachial (subdiscal) cell closed postero-apically usually before recurrent vein (m-cu). Parallel vein (CU1a) usually interstitial, rarely arising from anterior third (behind middle) of vein closing brachial (subdiscal) cell anteriorly. Hind wing with three hamuli. Radial (marginal) cell without additional transverse vein (r). Medial (basal) cell narrow. Nervellus (cu-a) present. Submedial (subbasal) cell relatively short; first abscissa of mediocubital vein $(\mathrm{M}+\mathrm{CU})$ distinctly (0.4-0.6 times) shorter than second abscissa (1-M). Recurrent vein (m-cu) usually present and transparent, rarely almost indistinct.

Legs. Fore and middle tibiae with distinct, rather thick and short spines arranged in often single longitudinal row. Hind coxa always with distinct basoventral corner and tubercle. Hind femur with low dorsal protuberances. Hind basitarsus $0.5-0.6$ times as long as second-fifth segments combined.

Metasoma. Presenting six or seven dorsally visible tergites, but sixth tergite sometimes rather shortly protruding behind fifth one. First tergite not petiolate, wide and relatively short; its dorsope distinct, basolateral lobes small or very small. Acrosternite of first segment about 0.2 times as long as first tergite, its apical margin situated before level of spiracles. Second tergite always without basal area; often with apical lenticular area delineated anteriorly by deep curved furrow and suture posteriorly, sometimes this apical area absent. Second suture distinct, usually regularly curved and crenulate. Second-sixth tergites with separate laterotergites. Sixth or sometimes only fifth tergites often enlarged, usually longer than previous tergite, but not entirely covering following protruding apical segments; usually sculptured at least basally or entirely. Ovipositor usually shorter than metasoma.

\section{Diagnosis}

This genus belongs to the group of genera with at least six dorsally visible tergites. Rhaconotinus is very similar to Ipodoryctes Granger but differs from it by the always missing delineated basal area on the second metasomal tergite and the parallel vein (CU1a) of fore wing mainly interstitial.

\section{Composition}

This genus currently consists of two subgenera, Rhaconotinus s. str. and Hexarhaconotinus subgen. nov. The following species belong to the nominative subgenus: Rhaconotinus (Rhaconotinus) affinis (Belokobylskij \& Chen, 2004) comb. nov. (OR); Rh. (Rh.) andreii (Belokobylskij, 2001) 
comb. nov. (OR); Rh. (Rh.) antennalis (Szépligeti, 1913) comb. nov. (AF); Rh. (Rh.) austrochinensis nom. nov. (Rhaconotus chinensis Chen \& Shi, 2004 (December) nom. praeocc., not Rhaconotus chinensis Belokobylskij \& Chen, 2004 (June)) (OR); Rh. (Rh.) bidens (Belokobylskij, 2001) comb. nov. (OR); Rh. (Rh.) caboverdensis (Hedqvist, 1965) stat. et comb. nov. (AF); Rh. (Rh.) chinensis (Belokobylskij \& Chen, 2004) comb. nov. (OR); Rh. (Rh.) choenobivorus (Rohwer, 1918) comb. nov. (OR); Rh. (Rh.) concinnus (Enderlein, 1912) comb. nov. (OR); Rh. (Rh.) concolor (Szépligeti, 1908) comb. nov. (OR); Rh. (Rh.) dabacus (Belokobylskij, 2001) comb. nov. (OR); Rh. (Rh.) elegans (Belokobylskij, 2001) comb. nov. (OR); Rh. (Rh.) guineensis (Szépligeti, 1914) comb. nov. (AF); Rh. (Rh.) hei (Belokobylskij \& Chen, 2004) comb. nov. (OR); Rh. (Rh.) heterotrichus (Belokobylskij $\&$ Chen, 2004) comb. nov. (OR); Rh. (Rh.) insularis (Belokobylskij, 1988) comb. nov. (OR); Rh. (Rh.) intermedius (Belokobylskij \& Chen, 2004) comb. nov. (OR); Rh. (Rh.) ipodoryctoides (Belokobylskij \& Chen, 2004) comb. nov. (OR); Rh. (Rh.) iterabilis (Belokobylskij \& Chen, 2004) (PA, OR); Rh. (Rh.) lacertosus (Chen \& Shi, 2004) comb. nov. (OR); Rh. (Rh.) luteosetosus (Belokobylskij \& Chen, 2004) comb. nov. (OR); Rh. (Rh.) menippus (Nixon, 1939) comb. nov. (= Rhaconotus decaryi Granger, 1949) (OR, AF); Rh. (Rh.) nadezhdae (Tobias \& Belokobylskij, 1981) (PA, OR); Rh. (Rh.) orientalis (Szépligeti, 1913) comb. nov. (AF); Rh. (Rh.) perakensis (Belokobylskij, 2001) comb. nov. (OR); Rh. (Rh.) persimilis (Szépligeti, 1911) comb. nov. (AF); Rh. (Rh.) philippinensis (Belokobylskij, 2001) comb. nov. (OR); Rh. (Rh.) striatus (Szépligeti, 1911) comb. nov. (AF); Rh. (Rh.) tianmushanus (Belokobylskij \& Chen, 2004) comb. nov. (OR).

\section{Hosts}

Coleoptera: Cylas puncticollis Boheman, 1833, Hypolixus truncatulus (Fabricius, 1798), Lixus sp., Peloropus batatae Marshall, 1927, Pempheres affinis Faust, 1898 (Curculionidae).

Lepidoptera: Chilo suppressalis (Walker, 1863), Scirpophaga incertulas (Walker, 1863) S. nivella (Fabricius, 1794) (Crambidae); Sesamia inferens (Walker, 1856) (Noctuidae).

\section{Distribution}

Afrotropical, Oriental and Palaearctic regions.

\section{Remarks}

This genus was restored in status by Belokobylskij (2019) and Belokobylskij et al. (2019) based on phylogenetic affinity (Jasso-Martínez et al. 2019).

Rhaconotinus caboverdensis Hedqvist, 1965, stat. resurr. Figs 40-41

Rhaconotinus caboverdensis Hedqvist, 1965: 8.

Rhaconotinus caboverdensis - Shenefelt \& Marsh 1976: 1330. — Belokobylskij 1992: 907; 1994a: 340 (as valid species). - Yu et al. 2016 (as incorrect synonym of Rh. concinnus Enderlein). - JassoMartínez et al. 2019: 168.

\section{Material examined}

Holotype

CABO VERDE • ; ; “Ins. Cabo Verde, S. Tiago Lagoa, 15.2.54, Lindberg”, "Holotypus Rhaconotinus caboverdensis gen. n. sp. n., +, K.-J. Hedqvist det. 1962”, "Mus. Zool. H:fors, Spec. type. No. 5613, Rhaconotinus caboverdensis Hedqv."; ZMHU. 


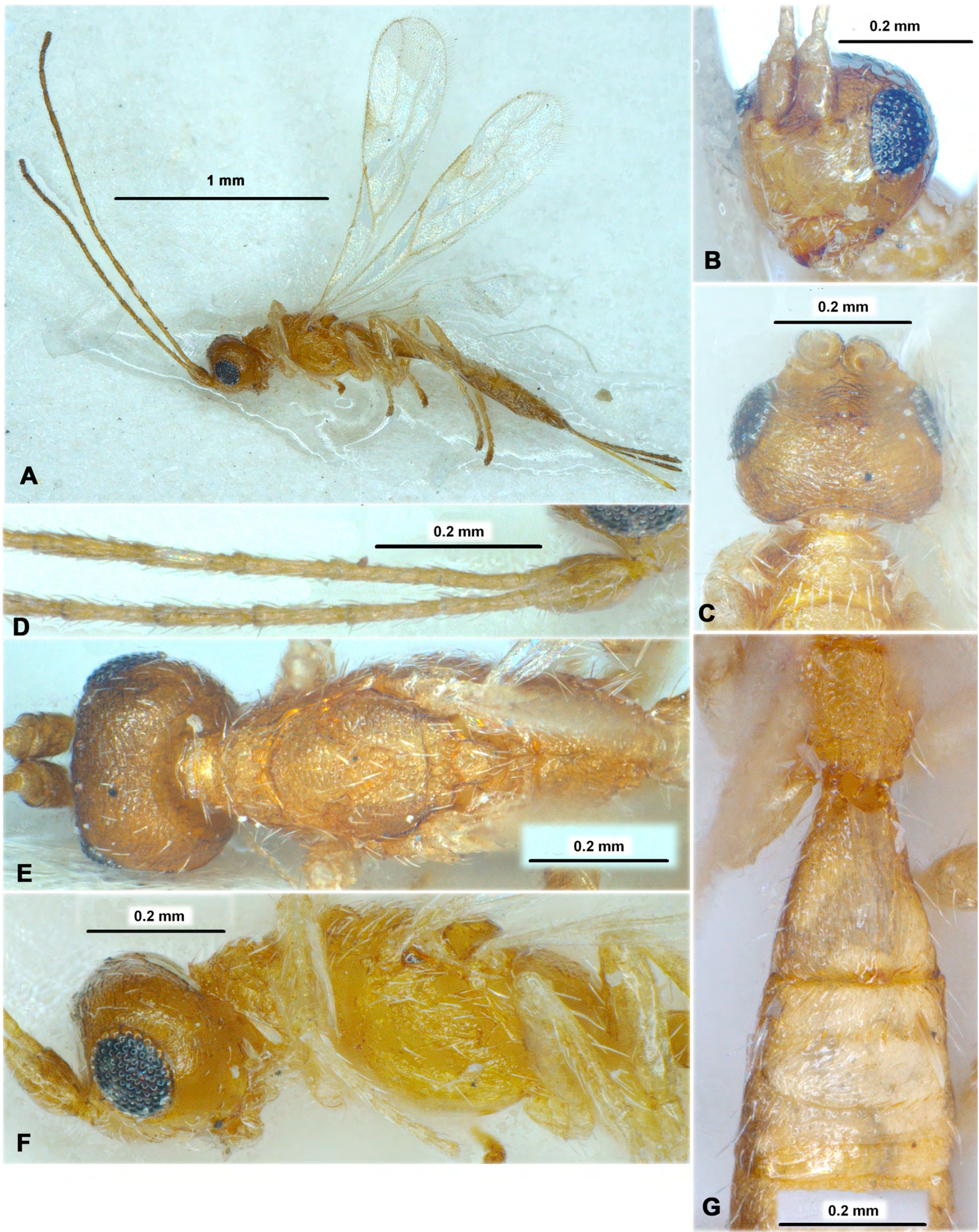

Fig. 40. Rhaconotinus caboverdensis Hedqvist, 1965, + , holotype, ZMHU. A. Habitus, lateral view. B. Head, fronto-lateral view. C. Head, dorsal view. D. Basal segments of antenna. E. Head and mesosoma, dorsal view. F. Head and mesosoma, lateral view. G. Propodeum and first to third metasomal tergites, dorsal view. 
BELOKOBYLSKIJ S.A. \& ZALDÍVAR-RIVERÓN A., Reclassification of the Rhaconotini
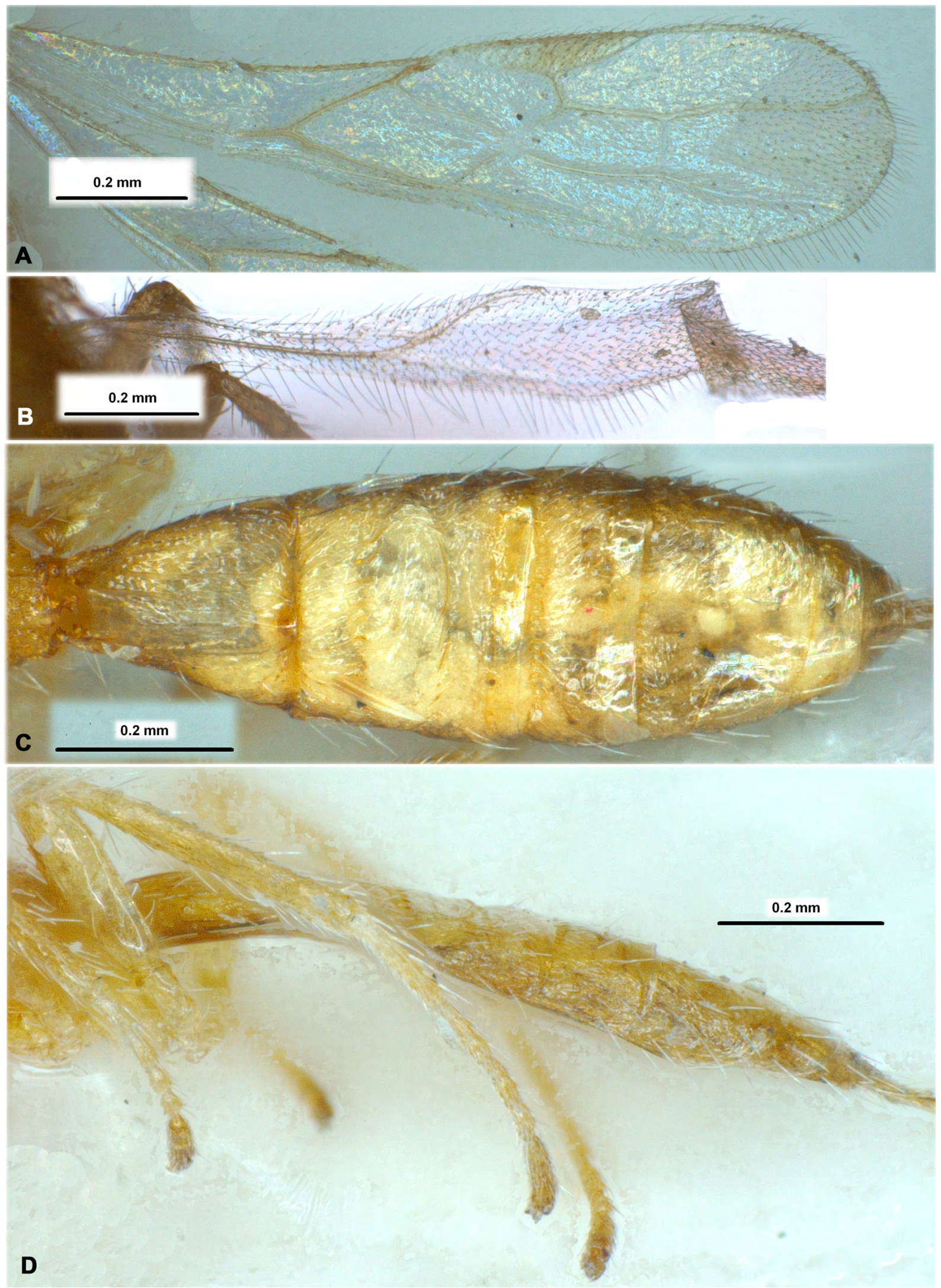

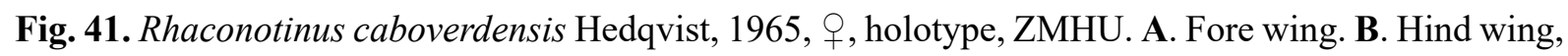
C. Metasoma, dorsal view. D. Metasoma and hind leg, lateral view. 


\section{Description}

\section{Female}

Measurements. Body length $1.7 \mathrm{~mm}$; fore wing length $1.5 \mathrm{~mm}$.

HEAD. Width 1.4 times its median length, 1.5 times width of mesoscutum. Head behind eyes (dorsal view) distinctly and roundly narrowed. Transverse diameter of eye 1.2 times length of temple. Ocelli small; POL 1.7 times OD, 0.6 times OOL. Eye without emargination opposite antennal socket, 1.3 times as high as broad. Malar space 0.7 times height of eye, equal to basal width of mandible. Face width 1.3 times height of eye and height of face and clypeus combined. Upper margin of clypeus situated before lower level of eyes. Hypoclypeal depression small and round, its width almost equal to distance from edge of depression to eye, 0.5 times width of face. Occipital carina not fused ventrally with hypostomal carina being obliterated upper base of mandible. Vertex distinctly convex. Head below eyes distinctly and roundly narrowed. Antennae slender, filiform, more than 18-segmented. Scapus 1.5 times as long as its maximum width. First flagellar segment about 5.0 times as long as its apical width. Subapical segments 4.0-4.3 times as long as width.

Mesosoma. Length 2.1 times its height. Pronotal carina weak, distances from carina to anterior margin of pronotum about 6.0 times distance from carina to posterior margin. Mesoscutum not highly and roundly elevated above pronotum (lateral view), almost as long as median width (dorsal view). Notauli entirely deep, rather wide, finely crenulate. Median lobe of mesoscutum with shallow median furrow. Prescutellar depression rather shallow, distinctly and roundly oblique postero-laterally, entirely distinctly sculptured, 0.5 times as long as the convex scutellum. Sternaulus (precoxal furrow) rather narrow, entirely granulate-coriaceous, running along almost entire length of lower part of mesopleuron. Prepectal carina below without widened lobes opposite fore coxae. Subalar depression rather deep, narrow, crenulate and fine additional reticulation between crenulae. Metanotum with very short and subpointed tooth directed backwards (lateral view). Metapleural lobe short, wide, subpointed apically, without dense pubescence.

WINGS. Fore wing 4.0 times its maximum width. Metacarpus (1-R1) weakly longer than pterostigma. First radial abscissa (r) forming almost straight angle with second abscissa (3-SR). Second radial abscissa (3-SR) 2.7 times as long as first abscissa (r), 0.4 times as long as the weakly sinuate third abscissa (SR1), 1.6 times as long as first radiomedial vein (2-SR). Second radiomedial (submarginal) cell rather long, weakly widened distally, about 3.0 times as long as wide, 1.2 times as long as brachial (subdiscal) cell. First medial abscissa (1-SR+M) weakly sinuate. Discoidal (discal) cell 2.5 times as long as maximum width. Mediocubital vein (M+CU1) weakly curved to anal vein (2-1A) in distal half. Distance between nervulus (cu-a) and basal (1-M) vein 1.3 times nervulus (cu-a) length. Brachial (subdiscal) cell narrow, gently-roundly closed distinctly before level of recurrent vein; posterior bulla and posterior abscissa of longitudinal anal vein (behind brachial vein (CU1b)) absent. Hind wing about 5.0 times as long as wide. First costal abscissa $(\mathrm{C}+\mathrm{SC}+\mathrm{R}) 0.5$ times as long as second abscissa (1-SC+R). First abscissa of mediocubital vein $(\mathrm{M}+\mathrm{CU})$ about 0.4 times as long as second abscissa (1-M).

LeGs. Hind femur with distinct dorsal protuberance, length of femur 2.8 times its maximum width. Hind tarsus almost as long as hind tibia. Hind basitarsus 0.5 times length of second-fifth segments combined. Second tarsal segment 0.55 times as long as basitarsus, 1.1 times as long as fifth segment (without pretarsus). 
Metasoma. Length 1.2 times as long as head and mesosoma combined, with six dorsally visible tergites. First tergite without subbasal lateral processes. Maximum width of first tergite about twice its basal width; its length almost equal to apical width, 1.1 times length of propodeum. Median length of second tergite 0.7 times its basal width, 1.8 times length of third tergite; median length of apical area 1.4 times length of rest part of tergite. Second suture deep and rather wide. Fifth tergite straight in posterior margin, without median emargination and postero-ventral lobes. Fifth tergite 1.4 times as long as fourth tergite, almost twice as long as than third or sixth tergites. Ovipositor sheath 0.6 times as long as metasoma, 1.4 times as long as hind tibia, 0.9 times as long as mesosoma, 0.4 times as long as fore wing.

SCUlPTURE AND PUBESCENCE. Vertex distinctly densely granulate, with additional distinct, weakly undulate and rather dense transverse striae; frons densely granulate and with distinct curved striae directed from lateral sides towards middle; face medially finely granulate-coriaceous, smooth or almost smooth widely laterally; temple granulate with curved striation, finely granulate to coriaceous or smooth in lower third. Mesoscutum entirely densely and distinctly granulate, without rugosity along notauli and laterally, with short rugosity in medio-posterior 0.3 of mesoscutum. Scutellum mostly smooth, coriaceous anteriorly. Mesopleuron entirely densely granulate. Metapleuron rugose-reticulate with granulation. Propodeum small areolate-rugulose with granulation, with granulate and indistinctly delineated by carinae basolateral areas, without areola. Hind coxa mostly smooth, finely granulate dorsally. Hind femur coriaceous in upper half and almost smooth in lower half. First tergite with rather distinct, almost complete and weakly convergent dorsal carinae, distinctly coarsely striate with fine reticulation between striae, smooth in medio-basal 0.4 and in medio-apical small area. Second tergite basally coarsely and curvedly striate, its apical area finely and subtransversely striate. Third and fourth tergites in basal 0.3 (medially) - 0.7 (laterally) distinctly curvedly striate, almost transversely striate medially, smooth in apical parts. Fifth tergite rugulose-striate in basal $0.2-0.3$, finely reticulate to smooth on most part. Second-fourth tergites laterally almost entirely densely striate with reticulation. Vertex entirely with short, sparse and semi-erect pale setae. Mesoscutum with rather sparse, short and semi-erect setae arranged widely along notauli and narrowly marginally, with narrow glabrous median areas on all lobes. Mesopleuron almost entirely covered by sparse pale setae. Hind tibia dorsally with rather long, more or less dense and semi-erect pale setae; length of these setae 0.5-1.0 times maximum width of hind tibia.

Colour. Head light reddish brown, paler ventrally. Mesosoma yellowish brown. Metasoma yellow, reddish brown marginally. Antenna light reddish brown in basal 0.3 and reddish brown on remaining part. Palps yellow. Legs yellow, all femora brown or light brown in apical 0.6-0.8, apical segments of all tarsi dark brown. Ovipositor sheath black, reddish brown in basal 0.3 . Fore wing hyaline. Pterostigma light brown in apical $0.5-0.6$, pale yellow in basal $0.4-0.5$.

\section{Male \\ Unknown.}

\section{Distribution}

Republic of Cabo Verde.

\section{Remarks}

In world Catalogue by $\mathrm{Yu}$ et al. (2016), this species was erroneously considered as a synonym of Rhaconotus concinnus (Enderlein, 1912), giving an incorrect reference for this synonymization (Belokobylskij 1994a). Actually, in the latter study only Rhaconotinus was synonymised with Rhaconotus s. lat., whereas Rh. caboverdensis was only compared with Rh. concinnus. 


\section{Subgenus Hexarhaconotinus subgen. nov. urn:1sid:zoobank.org:act:D0729E6D-6753-4910-9B10-CDFC2C5E7CCC}

Rhaconotus hexatermus species group Belokobylskij, 2001: 134.

\section{Etymology}

From combination of the Greek word "hexa", which means "six", and the generic name "Rhaconotinus", since members of this subgenus have six visible metasomal tergites.

\section{Type species}

Rhaconotus hexatermus Belokobylskij, 1988

\section{Description (Figs 42-43)}

HEAD. Head transverse. Ocelli arranged in weakly obtuse or equilateral triangle with base weakly larger than or almost equal to its sides. Eyes glabrous. Occipital carina complete dorsally, obliterate below and not joining ventrally with hypostomal carina at distinct distance. Malar suture absent. Hypoclypeal depression medium size, subround. Postgenal bridge narrow. Scapus of antenna wide and rather short. First flagellar segment subcylindrical, almost straight, not shorter than second segment. Apical segment acuminate apically.

Mesosoma. Mesosoma not or weakly depressed dorso-ventrally and relatively long. Pronotum dorsally convex, with distinct pronotal carina submedially. Mesonotum usually not highly and gently-roundly elevated above pronotum; mainly granulate. Notauli complete, distinct anteriorly and shallow posteriorly, crenulate. Prescutellar depression rather long, with distinct median carina. Metanotum with short and subrounded median tooth (lateral view). Sternaulus (precoxal furrow) rather deep, long, running along anterior $0.8-0.9$ of lower part of mesopleuron. Propodeum with basolateral areas and often areola distinctly delineated by carinae; lateral tubercles short.

WiNGs. Wings not shortened. Pterostigma of fore wing relatively wide. Radial vein (r) arising almost from middle of pterostigma. Radial (marginal) cell not shortened. Second radiomedial (submarginal) cell relatively short. Recurrent vein (m-cu) postfurcal. Nervulus (cu-a) postfurcal. Parallel vein (CU1a) usually interstitial, but rarely not interstitial and arising from anterior 0.25 of distal margin of brachial (subdiscal) cell. Brachial (subdiscal) cell subvertically closed postero-apically before or on level of recurrent vein $(\mathrm{m}-\mathrm{cu})$ and with marked antero-posteriorly corner. In hind wing, first abscissa of mediocubital vein $(\mathrm{M}+\mathrm{CU})$ 0.4-0.6 times as long as second abscissa (1-M).

Legs. Hind coxa with distinct basoventral corner and tubercle. Hind femur rather wide, with low dorsal protuberance. Hind basitarsus $0.6-0.7$ times as long as second-fifth segments combined.

Metasoma. First tergite not petiolate, rather long and distinctly widened toward apex. Second tergite without basal and apical areas delineated by furrows and second suture. Second suture deep, complete and regularly curved, without sublateral breaks. Sixth tergite at least weakly enlarged, usually longer than previous tergite, entirely coarsely sculptured, usually covered following apical segments, posteriorly evenly curved, without medial emargination. Ovipositor sheath not longer than metasoma.

\section{Diagnosis}

This subgenus differs from the nominative subgenus Rhaconotinus s. str. by the absence of apical area on the second metasomal tergite bordered by second suture and deep anterior transverse additional furrow. 


\section{Composition}

Rhaconotinus (Hexarhaconotinus) ashmeadi (Baker, 1917) comb. nov. (OR); Rh. (H.) brevitergum Belokobylskij, 2001 comb. nov. (OR); Rh. (H.) caophongus (Belokobylskij, 2001) comb. nov. (OR); Rh. (H.) hexatermus (Belokobylskij, 1988) comb. nov. (OR); Rh. (H.) fujianus (Belokobylskij \& Chen, 2004) comb. nov. (OR); Rh. (H.) icterus (Shi \& Chen, 2004) comb. nov. (OR); Rh. (H.) longi (Belokobylskij, 2001) comb. nov. (OR); Rh. (H.) micholitzi (Belokobylskij, 2001) comb. nov. (AU); Rh. (H.) rugosus (Chen \& Shi, 2004) comb. nov. (OR); Rh. (H.) staudingeri (Belokobylskij, 2001) comb. nov. (OR).

\section{Distribution}

Australasian and Oriental regions.

Rhaconotinus (Hexarhaconotinus) hexatermus (Belokobylskij, 1988) comb. nov. Figs $42-43$

Rhaconotus hexatermus Belokobylskij, 1988a: 98.

Rhaconotus hexatermus - Belokobylskij 2001: 134. - Belokobylskij \& Chen 2004: 354. - Yu et al. 2016.

\section{Material examined}

\section{Holotype}

CHINA • + ; "China, Guangzhou (Canton), border of forest, cytrous plantation, Sugonyaev (leg.), 10.XI 1986”; ZISP.

\section{Description}

\section{Female}

MEASUREMENTs. Body length $2.3 \mathrm{~mm}$; fore wing length $1.9 \mathrm{~mm}$.

HEAD. Width 1.5 times its median length, 1.2 times width of mesoscutum. Head behind eyes (dorsal view) distinctly and roundly narrowed. Transverse diameter of eye 2.2 times length of temple. Ocelli small, in almost equilateral triangle; POL almost equal to OD, 0.3 times OOL. Eye with weak emargination opposite antennal socket, 1.1 times as high as broad. Malar space 0.4 times height of eye, almost equal to basal width of mandible. Face width 1.3 times height of eye and 1.7 times height of face and clypeus combined. Upper margin of clypeus situated upper lower level of eyes. Hypoclypeal depression small and round, its width $0.6-0.7$ times distance from edge of depression to eye, 0.3 times width of face. Occipital carina not fused ventrally with hypostomal carina being obliterated upper base of mandible. Vertex weakly convex. Head below eyes distinctly and weakly-roundly narrowed. Antennae slender, weakly setiform, 25-segmented, 1.4 times as long as body. Scapus 1.4 times as long as its maximum width. First flagellar segment 4.5 times as long as its apical width, 1.1 times as long as second segment. Penultimate segment 4.7 times as long as wide, 0.8 times as long as first flagellar segment and 0.9 times as long as apical segment; the latter acuminate apically.

Mesosoma. Length 2.1 times its height. Pronotal carina distinct, distances from carina to anterior margin of pronotum subequal to distance from carina to posterior margin. Mesoscutum weakly, not highly and gently-roundly elevated above pronotum (lateral view), 0.8 times as long as maximum width (dorsal view). Notauli deep anteriorly and shallow in posterior 0.4 , rather wide, finely crenulate with granulation. Median lobe of mesoscutum with shallow median furrow. Prescutellar depression rather shallow, rather long, distinctly and roundly widened postero-laterally, with high median carina, entirely 


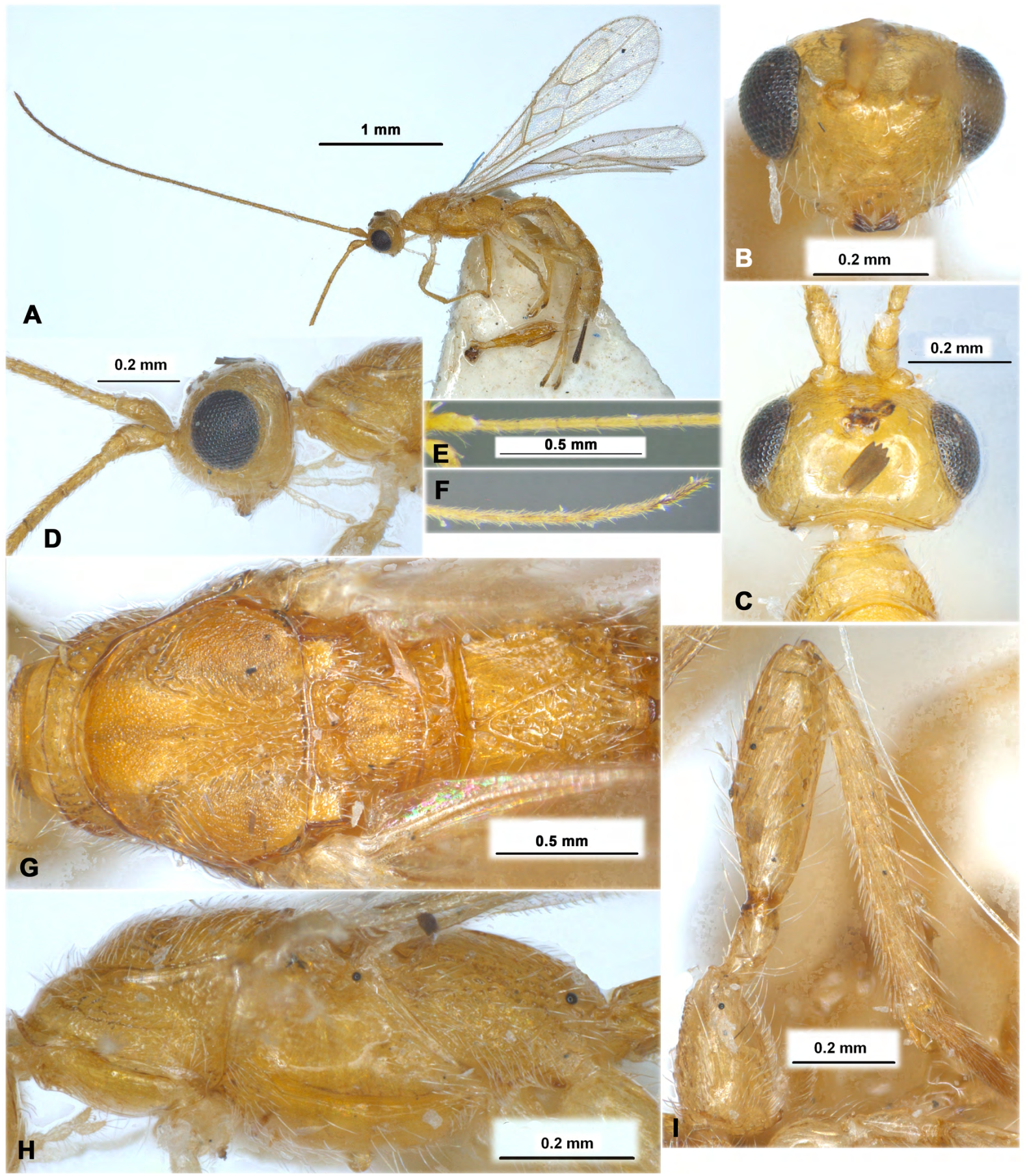

Fig. 42. Rhaconotinus (Hexarhaconotinus) hexatermus (Belokobylskij, 1988) comb. nov.,, , holotype, ZISP. A. Habitus, lateral view. B. Head, front view. C. Head, dorsal view. D. Head, lateral view. E. Basal segments of antenna. F. Apical segments of antenna. G. Mesosoma, dorsal view. H. Mesosoma, lateral view. I. Hind leg. 


\section{BELOKOBYLSKIJ S.A. \& ZALDÍVAR-RIVERÓN A., Reclassification of the Rhaconotini}
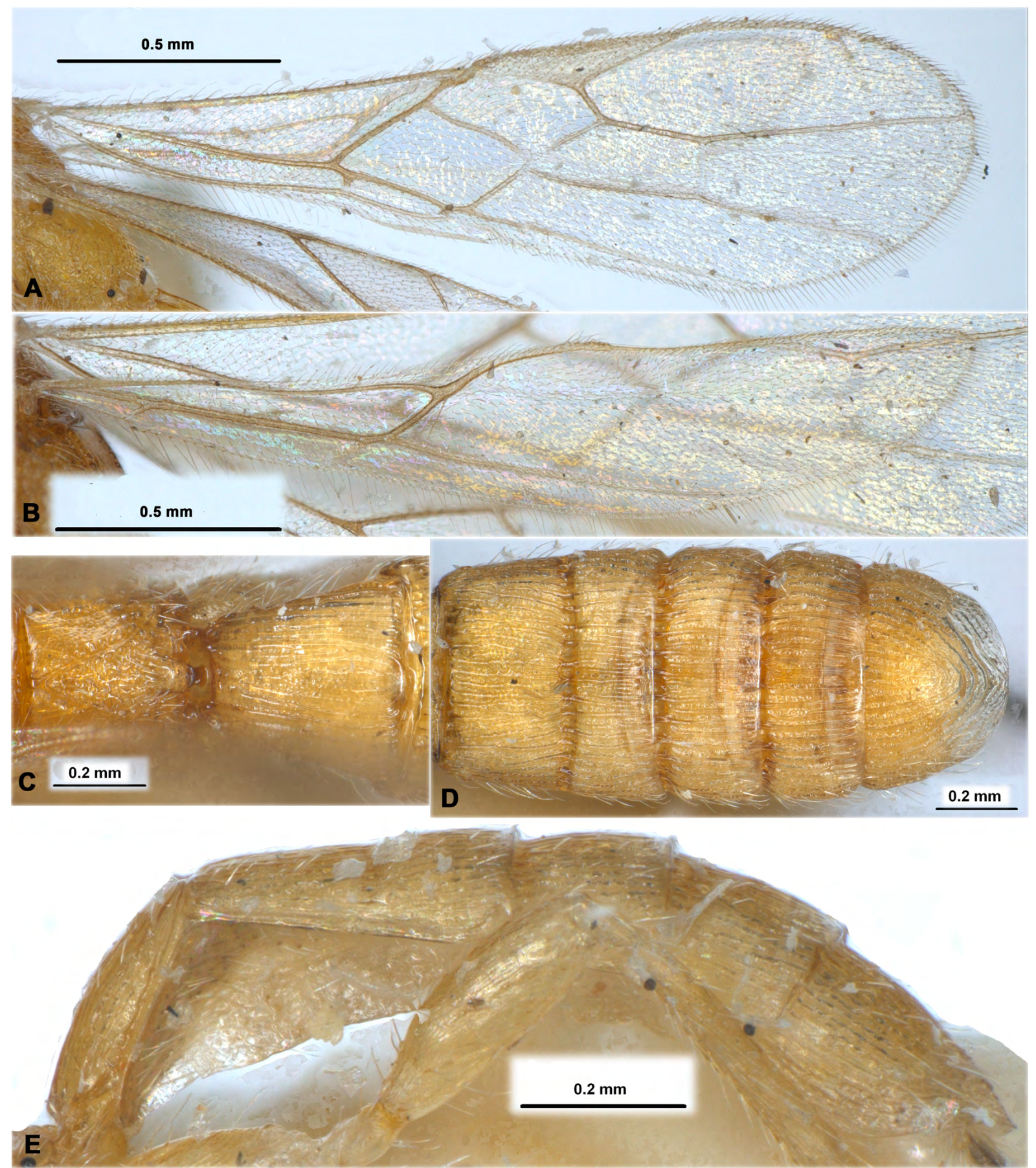

Fig. 43. Rhaconotinus (Hexarhaconotinus) hexatermus (Belokobylskij, 1988) comb. nov., +, holotype, ZISP. A. Fore wing. B. Hind wing. C. Propodeum and first metasomal tergite, dorsal view. D. Metasoma without first tergite, dorsal view. E. Metasoma, lateral view. 
rugulose, 0.6 times as long as weakly convex and transverse scutellum. Sternaulus (precoxal furrow) rather narrow, almost entirely smooth, running along almost entire length of lower part of mesopleuron. Prepectal carina below without widened lobes opposite fore coxae. Subalar depression shallow, rather narrow, finely transverse striate and partly with reticulation. Metanotum with very short and rounded dorsomedial tooth (lateral view). Metapleural lobe relatively long, wide, subrounded apically, without dense pubescence.

Wings. Fore wing 3.6 times its maximum width. Metacarpus (1-R1) 1.4 times as long as pterostigma. First radial abscissa (r) forming distinctly obtuse angle with second abscissa (3-SR). Second radial abscissa (3-SR) 2.5 times as long as first abscissa (r), 0.4 times as long as the straight third abscissa (SR1), 1.4 times as long as first radiomedial vein (2-SR). Second radiomedial (submarginal) cell medium-sized, not widened distally, 3.0 times as long as wide, 1.8 times as long as brachial (subdiscal) cell. First medial abscissa (1-SR+M) distinctly sinuate. Discoidal (discal) cell 2.2 times as long as maximum width. Mediocubital vein (M+CU1) very weakly curved to anal vein (2-1A) in distal half. Distance between nervulus (cu-a) and basal (1-M) vein 0.7 times nervulus (cu-a) length. Brachial (subdiscal) cell relatively narrow, gently-roundly closed rather distinctly before level of recurrent vein (m-cu); posterior bulla absent, posterior abscissa of longitudinal anal vein shortly developed. Hind wing 5.2 times as long as wide. First costal abscissa $(\mathrm{C}+\mathrm{SC}+\mathrm{R}) 0.5$ times as long as second abscissa $(1-\mathrm{SC}+\mathrm{R})$. First abscissa of mediocubital vein (M+CU) 0.5 times as long as second abscissa (1-M). Recurrent vein (m-cu) unsclerotised, antefurcal, strongly curved towards base of wing.

LEGS. Hind femur with very low and wide dorsal protuberance, length of femur 2.9 times its maximum width. Hind tarsus almost as long as hind tibia. Hind basitarsus 0.6 times length of second-fifth segments combined. Second tarsal segment 0.6 times as long as basitarsus, 1.3 times as long as fifth segment (without pretarsus).

Metasoma. Length 1.2 times as long as head and mesosoma combined, with six dorsally visible tergites. First tergite without subbasal lateral processes. Maximum width of first tergite 2.2 times its basal width; length 1.1 times its apical width, 1.25 times length of propodeum. Median length of second tergite 0.7 times its basal width, 1.8 times length of third tergite. Second suture deep, narrow, weakly evenly curved. Sixth tergite strongly convex in posterior margin, without median emargination and posteroventral lobes. Sixth tergite 1.3 times as long as fifth tergite, 1.5 times as long as third or sixth tergites. Ovipositor sheath 0.3 times as long as metasoma, 0.6 times as long as hind tibia, 0.5 times as long as mesosoma, 0.2 times as long as fore wing.

SCULPTURE AND PUBESCENCE. Vertex almost entirely smooth; frons finely granulate and with dense oblique aciculation; face almost entirely finely and densely granulate; temple smooth. Mesoscutum entirely densely granulate, without rugosity along notauli and laterally, with narrow area with rugosity in its medio-posterior 0.3. Scutellum finely granulate. Mesopleuron mostly smooth. Metapleuron rugosereticulate. Propodeum in basolateral areas rugulose-coriaceous, areola large and wide, rugose-reticulate, beginning from anterior 0.2 of propodeum, 1.3 times as long as maximum width. Hind coxa rugulose dorsally, mostly smooth. Hind femur coriaceous in upper half and almost smooth in lower half. First to fifth tergites distinctly densely longitudinally striate with fine reticulation between striae, fourth and fifths tergite almost smooth in narrow posterior areas. Sixth tergite entirely curvedly striate with dense reticulation. Second-fourth tergites laterally almost entirely densely striate with reticulation. Vertex almost entirely with short, very sparse and semi-erect pale setae. Mesoscutum entirely with dense, short and semi-erect yellow setae. Mesopleuron mostly glabrous. Hind tibia dorsally with rather long, relatively sparse and semi-erect yellow setae; length of these setae $0.8-1.0$ times maximum width of hind tibia. 
Colour. Body entirely yellow. Antenna yellow in basal third, light reddish brown in medial half and reddish brown to brown apically. Palps pale yellow. Legs yellow, apical segments of all tarsi brown to dark brown. Ovipositor sheath entirely black. Fore wing hyaline. Pterostigma entirely yellow.

Male

Unknown.

Distribution

China, Vietnam.

Genus Rhaconotus Ruthe, 1854

Rhaconotus Ruthe, 1854: 349.

Hedysomus Foerster, 1863: 238 (type species Hedysomus elegans Foerster, 1863, by monotypy; MHBG; examined).

Hormiopterus Giraud, 1869: 478 (type species Hormiopterus olivieri Giraud, 1869, by monotypy; MNHN; examined).

Rhadinogaster Szépligeti, 1908: 223 (type species Rhadinogaster testacea Szépligeti, 1908, by monotypy; HNHM; examined).

Aptenobracon Marsh, 1965: 675 syn. nov. (type species Aptenobracon formicoides Marsh, 1965, by monotypy and original designation; USNM; examined: Fig. 46).

Pararhacon Belokobylskij, 2005: 208 (as subgenus) (type species Rhaconotus (Pararhacon) haeselbarthi Belokobylskij, 2005, by monotypy and original designation; SSMG; examined).

Rhaconotus - Nixon 1941: 473. — Granger 1949: 126. — Marsh 1965: 694; 1976: 389; 2002: 180. — Shenefelt \& Marsh 1976: 1334. — Belokobylskij \& Tobias 1986: 46. — Belokobylskij 1990a: 145; 1994: 340; 2001: 102. — Belokobylskij \& Chen 2004: 319. — Chen \& Shi 2004: 35. - Yu et al. 2016. - Jasso-Martínez et al. 2019: 165.

Hedysomus - Shenefelt \& Marsh 1976: 1335. — Yu et al. 2016.

Hormiopterus - Shenefelt \& Marsh 1976: 1335. — Yu et al. 2016.

Rhadinogaster - Shenefelt \& Marsh 1976: 1335. — Yu et al. 2016.

Aptenobracon - Yu et al. 2016. — Jasso-Martínez et al. 2019: 165.

Rhaconotus (Pararhacon) - Yu et al. 2016.

\section{Type species}

Rhaconotus aciculatus Ruthe, 1854.

\section{Description (Figs 44-48)}

HEAD. Head not depressed, high, rather transverse. Ocelli arranged in obtuse triangle with base larger than sides. Frons not concave and without median keel or furrow. Eyes glabrous or sometimes with very short and sparse setae. Occipital carina dorsally complete, obliterate below and not joining ventrally with hypostomal carina at least at short distance. Malar suture absent. Clypeal suture distinct and complete. Hypoclypeal depression medium size or small, subround or oval. Postgenal bridge present and narrow. Palps usually rather long; maxillary palps 6-segmented, labial palps 4-segmented, its third segment not shortened. Scapus more or less wide, relatively long, without apical lobe and basal constriction, its ventral margin (lateral view) not longer than dorsal margin. First flagellar segment subcylindrical, usually at least weakly longer than second segment. Apical segment acuminate apically and without spine. 
Mesosoma. Mesosoma not or only weakly depressed and relatively long. Pronotum dorsally more or less distinctly convex, usually with distinct pronotal carina; pronope absent; propleural dorsoposterior flange long and wide. Mesonotum not highly and gently roundly elevated above pronotum. Median lobe of mesonotum without median longitudinal furrow and without anterolateral corner. Notauli usually distinct and complete, sometimes shallow posteriorly. Prescutellar depression relatively long or rarely short, always with distinct median carina. Lateral longitudinal wing-like flanges on the level of prescutellar depression rather wide and high. Scutellum convex, often with distinct lateral carinae. Metanotum with short, wide and usually pointed median tooth (lateral view). Sternaulus (precoxal sulcus) more or less deep, narrow, long, often weakly sinuate. Prepectal carina distinct and complete, rather high below. Postpectal carina absent. Metapleural flange short, wide, rounded apically. Propodeum often with basolateral areas delineated by carinae, always without areola and other areas; lateral tubercles and propodeal bridge absent.

WINGS. Predominantly full-winged, but rarely strongly or completely reduced. Pterostigma of fore wing rather narrow and long. Radial vein (r) arising mostly from middle of pterostigma, but sometimes weakly before or behind it. Radial (marginal) cell usually not shortened, but rarely weakly or distinctly shortened. Both radiomedial veins (2-SR, r-m) present. Second radiomedial (submarginal) cell long or medium-sized, rarely shortened. Recurrent vein (m-cu) basically postfurcal, only sometimes interstitial or weakly antefurcal. Discoidal (discal) cell petiolate anteriorly, petiole (1-SR) short. Nervulus (cu-a) postfurcal. Brachial (subdiscal) cell gently curvedly closed postero-apically on or often before recurrent vein (m-cu), without antero-posterior corner. Parallel vein (CU1a) always interstitial. Transverse anal veins $(2 \mathrm{~A}, \mathrm{a})$ absent. Hind wing with three hamuli. Radial vein $(\mathrm{SR})$ arising from costal vein $(2-\mathrm{SC}+\mathrm{R})$ rather distant from basal vein (1r-m). Submedial (subbasal) cell short. First abscissa of mediocubital vein $(\mathrm{M}+\mathrm{CU})$ 0.3-0.5 times as long as second abscissa (1-M). Recurrent vein (m-cu) present and weakly sclerotised or rarely absent.

LeGs. Fore and middle tibiae with distinct slender spines arranged almost in single row. Middle tarsal segments relatively long. Hind coxa with distinct basoventral corner and tubercle. Fore and middle femora with distinct and wide dorsal protuberances. Hind femur relatively narrow, with low and wide dorsal protuberance. Hind basitarsus 0.6 times as long as second-fifth segments combined. Claws short and simple.

Metasoma. Metasoma always with five dorsally visible, hardly sclerotised and almost entire or sometimes at least basally sculptured tergites, with distinctly separated laterotergites (epipleura). First tergite not petiolate, middle length and rather narrow or wide. Acrosternite of first segment short, about $0.20-0.25$ times as long as first tergite, its apical margin situated before level of spiracles. Dorsope of first tergite deep and rather wide; present weak and directed down short basolateral process; spiracles situated in basal 0.3 of tergite. Second tergite movably connected with first tergite; without basal area; apical lenticular area usually absent or weakly delineated by furrow anteriorly. Second suture deep, complete and regularly curved. Third tergite without transverse depression or furrow. Fifth tergite more or less distinctly enlarged, usually longer than previous tergite, covered following apical segments (in normal condition). Ovipositor sheath often shorter than metasoma.

\section{Diagnosis}

This is the type genus of the tribe Rhaconotini, and it characterises by having the following combination of morphological features: mesosoma of female always with only five dorsally visible tergites, fifth tergite almost always enlarged, at least basally sculptured and entirely or predominantly covered following apical tergites (in males sometimes apical metasomal tergites more or less distinctly projected); first and second tergites never immobile fused; recurrent vein (m-cu) of fore wing almost mostly postfurcal and falling into the second radiomedial (submarginal) cell (except subgenus Pararhacon); second 
BELOKOBYLSKIJ S.A. \& ZALDÍVAR-RIVERÓN A., Reclassification of the Rhaconotini

radiomedial vein (r-m) present; parallel vein (CU1a) always interstitial (very rarely wings strongly shortened or absent); propodeum always without delineated areola, but often with basolateral areas.

\section{Composition}

This genus currently consists of two subgenera, Pararhacon Belokobylskij, 2005 and Rhaconotus s. str. The following species belong to the nominative subgenus: Rhaconotus (Rhaconotus) aciculatus Ruthe, 1854 (Rh. cerdai Docavo Alberti, 1960; Rh. major Tobias, 1964) (PA); Rh. (Rh.) acmaeoderellae Belokobylskij, 1990 (PA); Rh. (Rh.) alpicola (Szépligeti, 1914) (AF); Rh. (Rh.) arabicus Belokobylskij, 2001 (PA); Rh. (Rh.) assander Nixon, 1943 (AF); Rh. (Rh.) atratus Marsh, 1976(NA); Rh. (Rh.) atys Nixon, 1941 (AF); Rh. (Rh.) badius Marsh, 1976 (NA); Rh. (Rh.) barri Marsh, 1976 (NA); Rh. (Rh.) bekilyensis Granger,1949 (AF); Rh. (Rh.) bicoloricornis (Granger,1949) comb. nov. (AF); Rh. (Rh.) bidentatus Granger, 1949 (AF); Rh. (Rh.) bisulcus Chen \& Shi, 2004 (OR); Rh. (Rh.) brachypterus (Hesse, 1934) (AF); Rh. (Rh.) brasiliensis Belokobylskij \& Zaldivar-Riverón, 2015 (NT); Rh. (Rh.) brevicaudus Marsh, 1976 (NA); Rh. (Rh.) canadensis Marsh, 1976 (NA); Rh. (Rh.) capensis (Brues, 1924) (AF); Rh. (Rh.) carinatus Polaszek, 1994 (AF); Rh. (Rh.) caudatus (Szépligeti, 1914) (AF); Rh. (Rh.) chrysochaitus Marsh, 2002 (NT); Rh. (Rh.) colegiomadridi Belokobylskij \& Zaldivar-Riverón, 2015 (NT); Rh. (Rh.) costaricensis Marsh, 2002 (NT); Rh. (Rh.) cressoni Musebeck \&Walkley, 1951 (Hormius aciculatus Cresson, 1872) (NA, NT); Rh. (Rh.) dentatus (Brues, 1924) (AF); Rh. (Rh.) elegans (Foerster, 1863) (PA); Rh. (Rh.) emarginatus Marsh, 2002 (NT); Rh. (Rh.) excavatus Belokobylskij, 2001 (OR); Rh. (Rh.) fasciatus (Ashmead, 1893) (NA); Rh. (Rh.) flavipes (Szépligeti, 1911) (AF); Rh. (Rh.) formicoides (Marsh, 1965) comb. nov. (NA); Rh. (Rh.) fuscipennis (Szépligeti, 1914) (AF); Rh. (Rh.) graciliformis (Viereck, 1911) (NA); Rh. (Rh.) hispanicus Belokobylskij, 2001 (PA); Rh. (Rh.) hyperion Nixon, 1941 (AF); Rh. (Rh.) hypolixi Nixon, 1941 (OR); Rh. (Rh.) iphias Nixon, 1941(AF); Rh. (Rh.) jakhontovi Yuldashev, 2004 (PA) (need verification of the type); Rh. (Rh.) kerzhneri Belokobylskij, 1985 (Rh. asiaticus Belokobylskij,1990, syn. nov.)(PA); Rh. (Rh.) longulus Belokobylskij, 1994 (PA); Rh. (Rh.) maculatus Belokobylskij, 2001 (OR); Rh. (Rh.) magnus Belokobylskij \& Chen, 2004 (OR); Rh. (Rh.) mahensis Wilkinson, 1931 (AF); Rh. (Rh.) manolus Nixon, 1941(AF); Rh. (Rh.) minor Szépligeti, 1914 (AF); Rh. (Rh.) niger Szépligeti, 1914 (AF); Rh. (Rh.) numitor Nixon, 1941 (AF); Rh. (Rh.) ochus Nixon, 1941 (AF); Rh. (Rh.) ollivieri Giraud, 1869 (PA); Rh. (Rh.) oriens Belokobylskij \& Chen, 2004 (PA, OR); Rh. (Rh.) phalarus Marsh, 1976 (NA, NT); Rh. (Rh.) pictipennis (Reinhard,1885) (PA); Rh. (Rh.) polycrates Nixon, 1941 (AF); Rh. (Rh.) republicanus Belokobylskij \& Zaldivar-Riverón, 2015 (NT); Rh. (Rh.) rufescens (Szépligeti, 1914) (AF); Rh. (Rh.) ruficollis Granger, 1949 (AF); Rh. (Rh.) rugosus Marsh, 2002 (NT); Rh. (Rh.) sabinae Belokobylskij \& Zaldivar-Riverón, 2015 (NT); Rh. (Rh.) sauteri (Watanabe, 1934) (R. cleanthes Nixon 1939) (OR); Rh. (Rh.) scaber Kokujev, 1900 (PA); Rh. (Rh.) sciron Nixon, 1941 (AF); Rh. (Rh.) scirpophagae Wilkinson, 1927 (OR, $\mathrm{AF}) ; R h .(R h$.) seyrigi Granger,1949 (AF); Rh. (Rh.) sinuatus Granger,1949 (AF); Rh. (Rh.) striativertex Granger, 1949 (AF); Rh. (Rh.) sudanensis Wilkinson, 1927 (AF); Rh. (Rh.) sulmo Nixon, 1941 (AF); Rh. (Rh.) tergalis Belokobylskij \& Chen, 2004 (OR); Rh. (Rh.) testacea Szépligeti, 1908 (Hormiopterus sulcativentris Enderlein, 1912; Rhaconoptus flavistigma Telenga, 1941; Rh. oryzae Wilkinson, 1929) (PA, OR); Rh. (Rh.) thestor Nixon, 1941 (AF); Rh. (Rh.) troilus Nixon, 1941 (AF); Rh. (Rh.) uzbekistanicus Yuldashev, 2004 (PA) (need verification of the type); Rh. (Rh.) yaoae Belokobylskij \& Chen, $2004(\mathrm{OR})$; Rh. (Rh.) zarudnyi Belokobylskij, 1990 (PA, OR).

\section{Hosts}

Coleoptera: Acmaeodera pulchella (Herbst, 1801), Acmaeoderella zeravshanica Volkovitsh, 1987; Anthaxia lgockii Obenberger, 1917, A. viridis (Linnaeus, 1758), Sphenoptera sp. (Buprestidae); Caryedon sp., Caryedon serratus (Olivier, 1790) (Chrysomelidae: Bruchinae); Conotrachelus sp., Hypolixus sp., Lixus concavus Say, 1831, L. lukjanovitschii Ter-Minasian, 1966, Trichobaris texana Le Conte, 1876 (Curculionidae); Mordellistena sp. (Mordellidae). 
Lepidoptera: Chilo auricilius Dudgeon, 1905, Ch. partellus (Swinhoe, 1885), Ch. saccharifagus (Bojer, 1856), Ch. suppressalis (Walker, 1863), Scirpophaga excerptalis (Walker, 1863), S. incertulas (Walker, 1863), S. nivella (Fabricius, 1794) (Crambidae); Oecocecis guyonella Guinee, 1870 (Gelechiidae); Busseola fusca (Fuller, 1901) (Noctuidae); Maliarpha separatella Ragonot, 1888 (Pyralidae).

\section{Distribution}

Afrotropical, Australasian, Nearctic, Neotropical, Oriental and Palaearctic regions.

\section{Remarks}

This is the largest genus of the tribe Rhaconotini, within which the most species of Rhaconotinus, Rhaconotus, Troporhaconotus and partly Ipodoryctes were previously described. The recent molecular phylogenetic study for the tribe Rhaconotini (Jasso-Martínez et al. 2019) consistently showed that this genus should be only restricted to species of Rhaconotus with five dorsally visible metasomal tergites.

The apterous North American genus Aptenobracon Marsh, 1965 (with type species A. formicoides: Fig. 46) was deeply nested in the phylograms of the above phylogenetic study together with the New World Rhaconotus species. Brachyptery (reduction of the wings plate and venation) has been already recorded in the genus Rhaconotus for two species, Rh. brachypterus (Hesse, 1934) and Rh. hispanicus Belokobylskij, 2001, where such reduction of the wing was associated with transformation of metasomal structures (decreased mesothorax and enlarged prothorax and metathorax together with propodeum). The above phylogenetic affinities lead us to conclude that all diagnostic characters for Aptenobracon are only due to its loss of wings. We therefore consider Aptenobracon Marsh, 1965 syn. nov. as a junior synonym of Rhaconotus Ruthe, 1854.

Study of rather numerous reared material for both sexes from Israel $[3+q$, Tarum, 25.II.1972, A. Goldstein leg., from Caryedon sp.; $2 \widehat{\jmath}$, Israel, II-III.1972, B.T. Southgate, "ex pods Prosopis farcta (Fabaceae), IV.1972”; 1 q, Natiu Ha- lamed Hey, 11.IV.1972, A. Goldstein leg., ex Caryedon sp. (Chrysomelidae: Bruchinae) (all in BMNH); 1 + , 1 , Yizre'el, 40 km SE of Haifa, 6.IV.1971, D. Gerling leg. (ZISP); 1 ○, Sede Terumot, 7 km S of Bet Shean, 29.X.1971, F. Goldstein leg., from Caryedon sp. (ZISP); 1 +, Israel, 25.II.1972, A. Belinsky leg., from Caryedon sp. (ZISP)], Iraq [1 \&, Abu-Ghraib, Baghdad, IV.1979, M. Abdul Rassoul leg. (ZISP); 1 ô, Hawiga, Kirkuk, 26.IV.1977, M. Abdul Rassoul leg. (ZISP)] and Iran [4 우, 4 $\lesssim \widehat{\jmath}$, Yazd Province, Yazd, coll 5.IV.2008 on Prosopis farcta (Fabaceae), A. Muhammadi leg.;

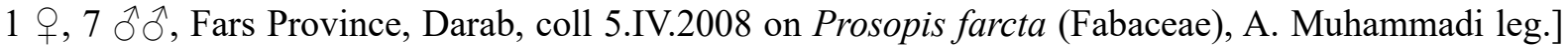
revealed that Rhaconotus asiaticus Belokobylskij, 1990 syn. nov. (was originally described only for females) is a junior synonym of $R h$. kerzhneri Belokobylskij, 1985, which was originally described only for males. Both sexes of this species are characterised by having a weakly shortened radial (marginal) cell of fore wing, but males additionally have a sharp sexual dimorphism, viz. the presence of double smoothed lateral areas on the second-fourth metasomal tergites (always absent in females).

The second metasomal tergite of several Rhaconotus species have transverse more or less lenticular apical area delineated anteriorly the transverse and usually curved sulcus (by different level of its development) and posteriorly by deep second suture. The anterior sulcus of the second tergite is a strongly variable structure (can be deep or shallow to very shallow), inclusive within a single species. The molecular phylogenetic study for the tribe Rhaconotini showed that the members of Rhaconotus s. str. with and without apical area on the second tergite are intermingled with other members of the genus (Jasso-Martínez et al. 2019). 
Rhaconotus (Rhaconotus) aciculatus Ruthe, 1854

Figs 44-45

Rhaconotus aciculatus Ruthe, 1854: 349.

Hormiopterus cerdai Docavo Alberti, 1960: 27.

Rhaconotus major Tobias, 1964: 179.

Rhaconotus aciculatus - Nixon 1941: 473. - Shenefelt \& Marsh 1976: 1335. — Belokobylskij 1990a: 145; 1998b: 69. - Belokobylskij \& Chen 2004: 354. - Yu et al. 2016. - Jasso-Martínez et al. 2019: 165.

Hormiopterus cerdai - Shenefelt \& Marsh 1976: 1336. — Belokobylskij 1990a: 145 (as synonym). — Yu et al. 2016 (as synonym).

Rhaconotus major - Shenefelt \& Marsh 1976: 1338. — Belokobylskij 1990a: 145 (as synonym). — Yu at al. 2016.

\section{Material examined}

Holotype

GERMANY • +; “Type H.T.” (round with red margin), "Ruthe Coll. 59.101”, "59.101. Germany”, "Rh. aciculatus m., Hasenheide, 15/6 53", "Rhaconotus m.", "Holotype" (round with red margin), "B.M. Type Hym.3c.1392”; BMNH.

\section{Other material}

SPAIN • 1 , , holotype of Rh. cerdai; "Ciudad Rial [Province], Pozuelo de Calatrava. 1903. La Fuente" [handwriting], "O+", "Rhaconotus cerdai n. sp., det. Docavo", "Tipo", "Rhaconotus + scaber Kok., det Papp J., 1983”, “MNCN, Cat. Tipos No 11534”; MNCN.

KAZAKHSTAN • 1 q, 1 , , holotype of Rh. major; "Koksengir [mountains], Karagand.[a Province], solenye ozera [salt Lakes], Lepidium latifolium [L. (Brassicaceae)], A. Ponomareva [leg.], 26.VI[1]959"; ZISP.

\section{Description}

Female (holotype of $R h$. aciculatus)

MEASUREMENTs. Body length $3.0 \mathrm{~mm}$; fore wing length $2.2 \mathrm{~mm}$.

HEAD. Width 1.4 times its median length, 1.4 times width of mesoscutum. Head behind eyes (dorsal view) distinctly and roundly narrowed. Transverse diameter of eye 1.7 times length of temple. Ocelli small, arranged in triangle with base 1.1 times its sides; POL 1.4 times OD, 0.4 times OOL. Eye glabrous, very weakly emarginated opposite antennal sockets, 1.2 times as high as broad. Malar space 0.6 times height of eye, 1.3 times basal width of mandible. Face width 1.2 times height of eye and 1.25 times height of face and clypeus combined. Malar suture absent. Upper margin of clypeus situated distinctly below lower level of eyes. Hypoclypeal depression round, its width 0.6 times distance from edge of depression to eye, 0.4 times width of face. Occipital carina complete dorsally, not fused ventrally with hypostomal carina being obliterated shortly upper base of mandible. Vertex distinctly convex. Head below eyes distinctly and weakly-roundly narrowed (front view). Antennae rather slender, filiform, 28-segmented, 1.2 times as long as body. Length of scapus 1.5 times its maximum width. First flagellar segment 4.8 times as long as its apical width, 1.1 times as long as second segment. Penultimate segment 3.0 times as long as wide, 0.5 times as long as first segment, 0.75 times as long as apical segment; the latter acuminate apically. 


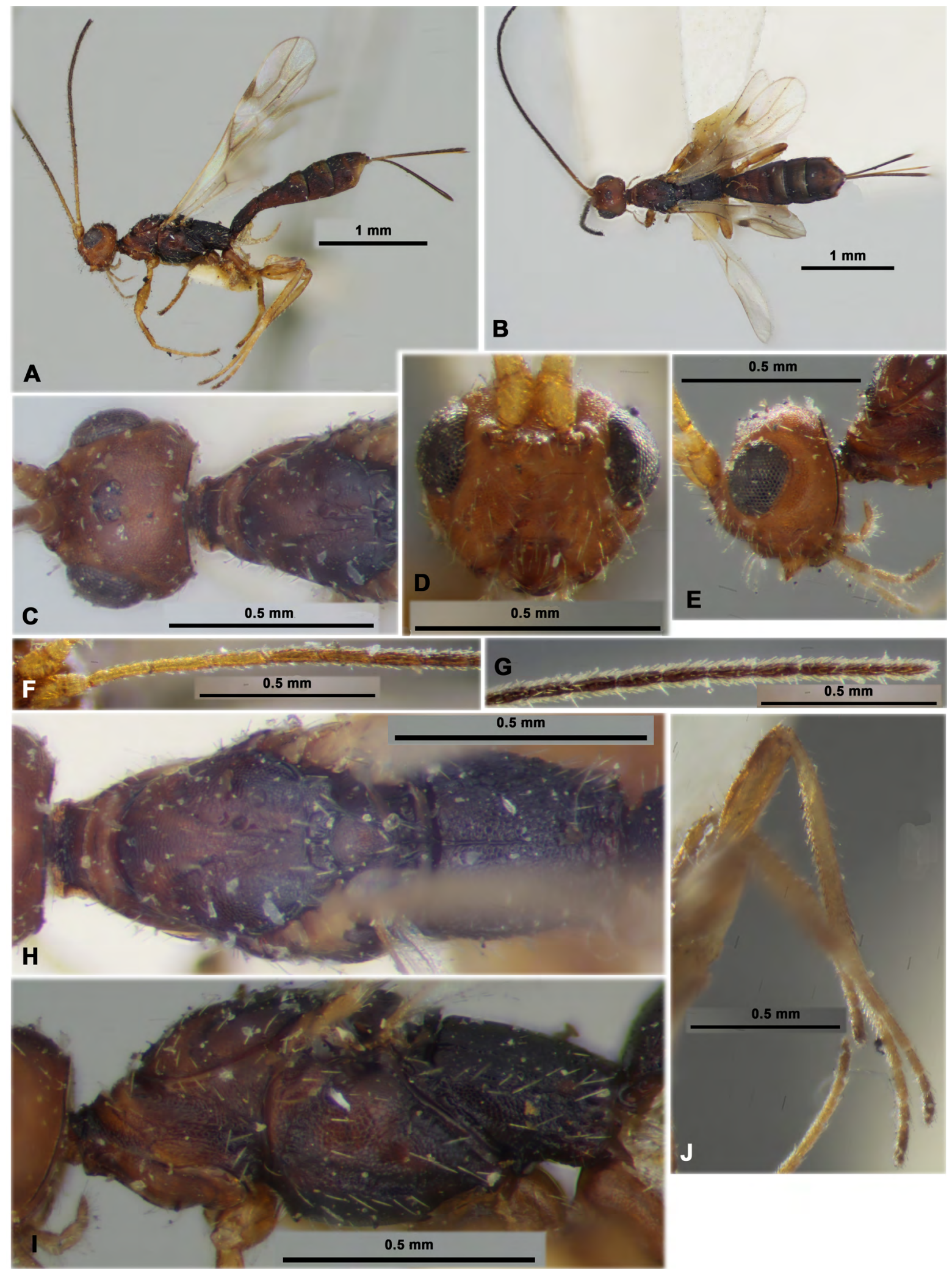

Fig. 44. Rhaconotus (Rhaconotus) aciculatus Ruthe, 1854, $q$, holotype, BMNH. A. Habitus, lateral view. B. Habitus, dorsal view. C. Head and mesoscutum, dorsal view. D. Head, front view. E. Head, lateral view. F. Basal segments of antenna. G. Apical segments of antenna. H. Mesosoma, dorsal view. I. Mesosoma, lateral view. J. Hind leg. 


\section{BELOKOBYLSKIJ S.A. \& ZALDÍVAR-RIVERÓN A., Reclassification of the Rhaconotini}
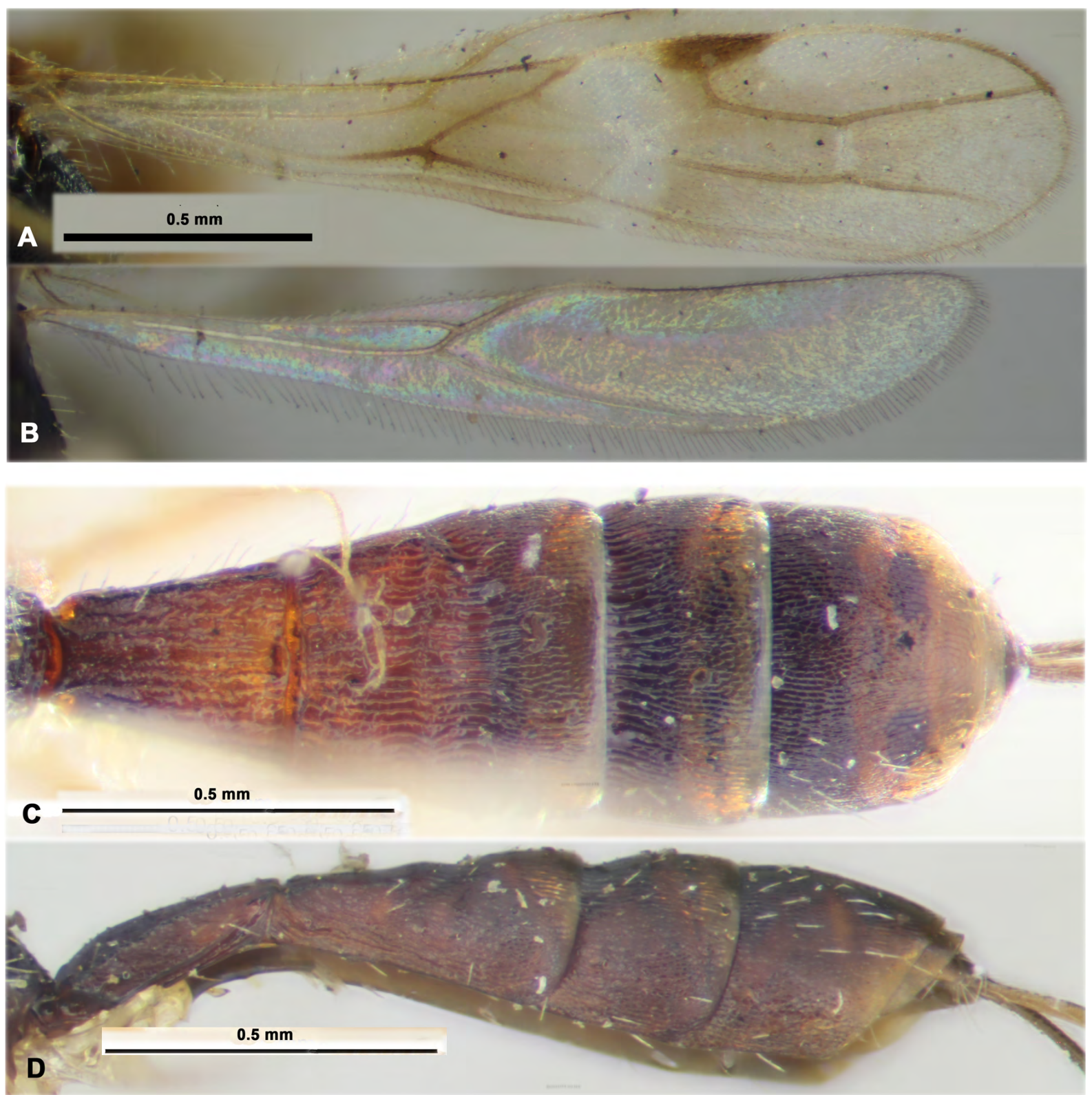

Fig. 45. Rhaconotus (Rhaconotus) aciculatus Ruthe, 1854,, , holotype, BMNH. A. Fore wing. B. Hind wing. C. Metasoma, dorsal view. D. Metasoma, lateral view. 


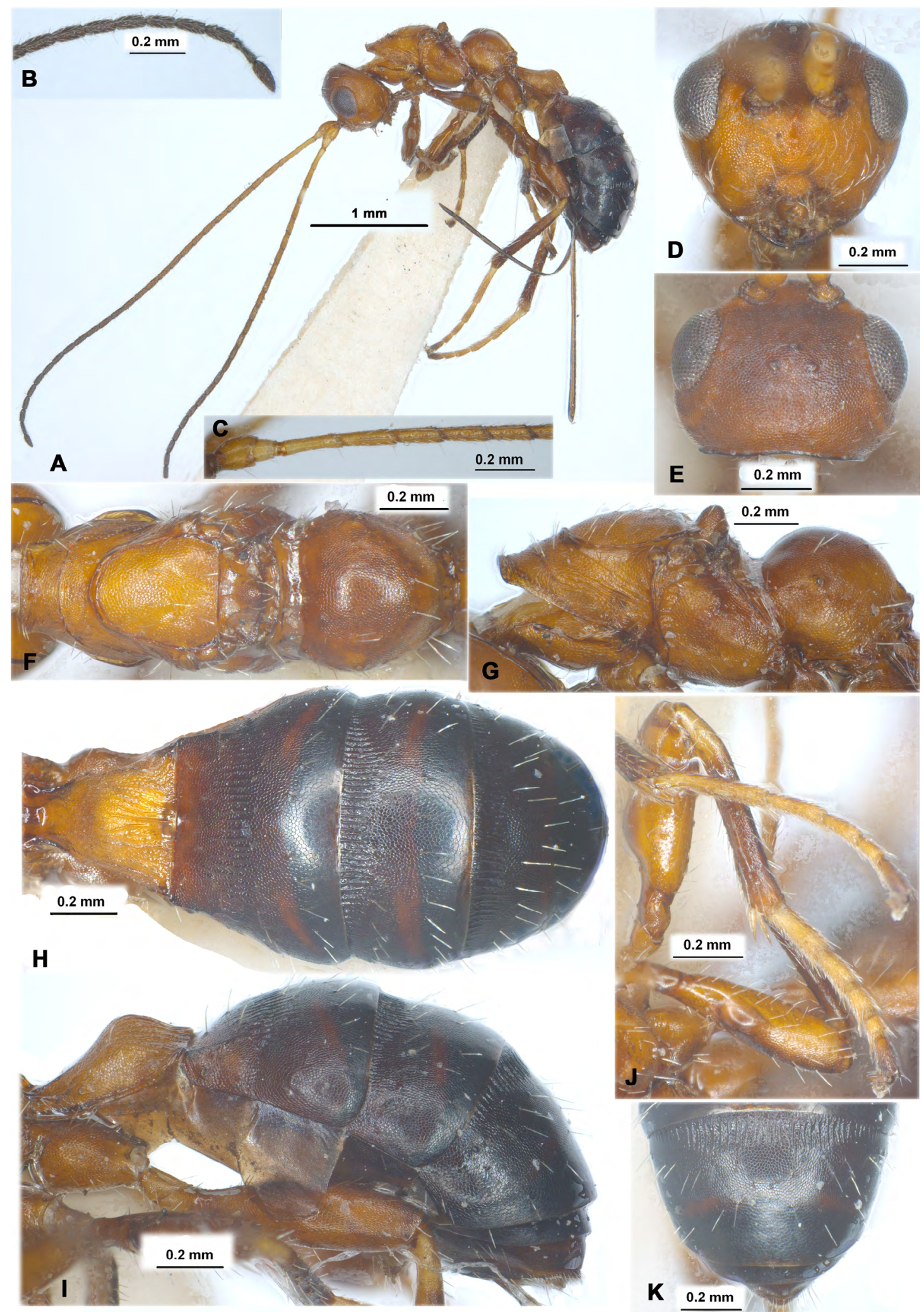

Fig. 46. Rhaconotus (Rhaconotus) formicoides (Marsh, 1965) comb. nov., + , holotype, USNM. A. Habitus, lateral view. B. Apical segments of antenna. C. Basal segments of antenna. D. Head, front view. E. Head, dorsal view. F. Mesosoma, dorsal view. G. Mesosoma, lateral view. H. Metasoma, dorsal view. I. Metasoma, lateral view. J. Hind leg. K. Apex of metasoma, dorsal view. 
Mesosoma. Length 2.3 times its height. Pronotal carina rather distinct, distances from carina to both sides of pronotum subequal. Mesoscutum rather highly and subroundly elevated above pronotum, 1.2 times as long as wide. Notauli more or less deep entirely, complete, rather wide, densely crenulate with fine granulation. Median lobe of mesoscutum without median furrow. Prescutellar depression rather deep, short, distinctly and roundly curved postero-laterally, with high median carina, finely rugulose, 0.3 times as long as convex scutellum. Sternaulus (precoxal furrow) shallow, rather narrow, weakly sinuate, finely crenulate with densely and rather fine granulation, running along almost entire lower part of mesopleuron. Prepectal carina without widened lobes opposite fore coxae. Subalar depression rather deep, narrow, sparsely crenulate with granulation. Metanotum with short, rather thick and pointed tooth directed backward (lateral view). Metapleural lobe rather long, wide, rounded apically, without dense pubescence. Mesopleural suture entirely and very shortly crenulate.

WINGS. Fore wing 4.6 times its maximum width. Radial (marginal) cell weakly shortened. Metacarpus (1-R1) 1.3 times as long as pterostigma. Radial vein (r) arising rather distinctly behind middle of pterostigma, from basal 0.55. First radial abscissa (r) forming obtuse angle with second abscissa (3-SR). Second radial abscissa (3-SR) 3.6 times as long as first abscissa (r), 0.6 times as long as the weakly curved third abscissa (SR1), 1.6 times as long as first radiomedial vein (2-SR). Second radiomedial (submarginal) cell rather long, not widened distally, 3.8 times as long as wide, 1.4 times as long as narrow brachial (subdiscal) cell. First medial abscissa (1-SR+M) weakly sinuate. Recurrent vein (m-cu) distinctly postfurcal. Mediocubital vein $(\mathrm{M}+\mathrm{CU} 1)$ more or less distinctly curved to anal vein $(2-1 \mathrm{~A})$ in distal half. Nervulus (cu-a) postfurcal, distance between nervulus (cu-a) and basal (1-M) vein 0.4 times nervulus (cu-a) length. Brachial (subdiscal) cell gently-roundly closed shortly before level of recurrent vein; posterior bulla and posterior abscissa of anal vein (2-1A) (behind brachial vein (CU1b)) absent. Hind wing 6.2 times as long as wide. First costal abscissa $(\mathrm{C}+\mathrm{SC}+\mathrm{R}) 0.5$ times as long as second abscissa $(1-\mathrm{SC}+\mathrm{R})$. First abscissa of mediocubital vein $(\mathrm{M}+\mathrm{CU}) 0.35$ times as long as second abscissa (1-M). Recurrent vein (m-cu) almost indistinct.

Legs. Hind coxa with distinct basoventral tubercle, 1.5 times as long as wide (with tubercle). Hind femur with very shallow dorsal protuberance, its length 3.3 times maximum width. Hind tarsus 1.1 times as long as hind tibia. Hind basitarsus 0.6 times combined length of second-fifth segments. Second tarsal segment 0.55 times as long as basitarsus, 2.1 times as long as fourth segment, 1.5 times as long as fifth segment (without pretarsus).

Metasoma. Length 1.1 times as long as head and mesosoma combined, with five dorsally visible tergites (very rarely following apical tergites shortly protruding behind fifth one, especially in male). First tergite with very short, pointed and directed ventrally subbasal lateral processes. Maximum width of first tergite about twice its basal width; its length 1.2-1.3 times apical width, 1.1 times length of propodeum. Second tergite without basal area, without or sometimes with weak, relatively wide and curved transverse subposterior furrow weakly separated apical sublenticular area (its median length 0.8 times length of remaining part of tergite). Median length of second tergite 0.8 times its basal width, 1.4 times length of third tergite. Second suture rather deep and narrow, almost straight medially and weakly curved laterally. Fifth tergite enlarged, weakly roundly convex in posterior margin, without median emargination and postero-ventral lobes. Fifth tergite 1.6 times as long as fourth tergite, 1.85 times as long as third tergite. Ovipositor sheath 0.7 times as long as metasoma, 1.3 times as long as hind tibia, 0.9 times as long as mesosoma, 0.45 times as long as fore wing.

SCULPTURE AND PUBESCENCE. Vertex densely distinctly granulate, often without rugulosity; frons densely granulate and without rugae; face densely and rather finely granulate; temple granulate almost entirely. Mesoscutum entirely densely granulate, often granulation anteriorly arranged in semicircular lines, without rugosity along notauli, with two sublongitudinal striae in medioposterior 0.3 of mesoscutum 
and short rugosity on it sides. Scutellum densely and rather finely granulate. Mesopleuron densely and entirely granulate-reticulate. Metapleuron densely granulate, additionally rugulose-striate in posterior half. Propodeum with large basolateral areas delineated by distinct or fine carinae, with distinct and long median and lateral carinae in basal half, densely granulate in anterior half and rugose-reticulate with granulation in posterior half. Hind coxa entirely densely granulate, without rugosity dorsally. Hind femur rather distinctly granulate-coriaceous, only coriaceous in lower half. First tergite with rather distinct and almost complete subparallel dorsal carinae, distinctly coarsely striate with ground reticulation between striae, densely rugulose mediobasally. Second tergite entirely coarsely and undulately striate with dense ground rugulosity. Third and fourth tergites in their basal 0.8 distinctly densely linearly striate with fine ground reticulation, smooth apically on narrow transverse stripe. Fifth tergite densely and rather finely striate in its basal $0.5-0.7$, densely and small reticulate-coriaceous posteriorly becoming finer towards apex, sometimes almost smooth apically. Second-fourth tergites laterally entirely densely granulatereticulate with dense and fine longitudinal striation. Vertex entirely with rather long, sparse, semi-erect pale setae, glabrous in medio-anterior half. Mesoscutum with sparse, rather short and semi-erect setae arranged rather widely along notauli and in a single row marginally, glabrous on wide median areas on all lobes. Mesopleuron glabrous medially. Hind tibia dorsally with short, rather dense and semi-erect pale setae; length of these setae 0.4-0.6 times maximum width of hind tibia.

CoLour. Head light reddish brown, darker dorsally; mesosoma light reddish brown or reddish brown partly, propodeum and metapleuron dark reddish brown to almost black; metasoma dark reddish brown, apical half of first tergites, entirely second and apical half of fifth reddish brown. Antenna dark reddish brown to black, reddish brown in basal quarter, two basal segments brownish yellow. Palps reddish brown. Legs brownish yellow or light reddish brown, tarsi (except for apical segments) yellow, hind femur and tibia apically infuscate. Ovipositor sheath black. Fore wing hyaline, with two large infuscate spots around basal vein (1-M) and second radiomedial (submarginal) cell. Pterostigma mainly dark brown, but yellow in its basal 0.4 .

\section{Distribution}

Palaearctic region (widely distributed in warm territories, from west to east parts).

Subgenus Pararhacon Belokobylskij, 2005

Rhaconotus (Pararhacon) Belokobylskij, 2005: 208.

Rhaconotus (Pararhacon) - Yu et al. 2016.

\section{Type species}

Rhaconotus (Pararhacon) haeselbarthi Belokobylskij, 2005

\section{Description (Figs 47-48)}

HEAD. Head transverse. Eyes with distinct, but sparse and short setae. Occipital carina complete, joining ventrally with hypostomal carina. Clypeal suture distinct and complete. Hypoclypeal depression rather small, subround. Postgenal bridge very narrow. Palps rather short. Scapus of antenna relatively wide and long. First flagellar segment almost straight, weakly longer than second segment. Apical segment pointed apically. Antennae brownish yellow to yellow in apical 0.3 .

Mesosoma. Pronotum with distinct pronotal carina situated medially. Mesonotum rather highly and roundly elevated above pronotum. Notauli complete and coarsely crenulate. Prescutellar depression rather long, with distinct median carina. Metanotum with short and subacuminate median tooth (lateral view). Sternaulus (precoxal furrow) deep and long, running along entire lower length of mesopleuron. 
Prepectal carina distinct and complete. Propodeum with basolateral areas not distinctly delineated by carinae, without areola; lateral tubercles distinct and thick.

WiNGs. Wings weakly shortened. Pterostigma of fore wing rather wide. Radial vein (r) arising distinctly behind middle of pterostigma. Radial (marginal) cell not shortened. Recurrent vein (m-cu) distinctly antefurcal. Nervulus (cu-a) postfurcal. Brachial (subdiscal) cell closed apically distinctly before recurrent vein (m-cu). Parallel vein (CU1a) interstitial. In hind wing, radial (marginal) cell almost parallel-sided. Submedial (subbasal) cell medium size; First abscissa of mediocubital vein $(\mathrm{M}+\mathrm{CU})$ distinctly shorter than second abscissa (1-M). Recurrent vein (m-cu) present and almost straight.

LEGS. Fore and middle tibiae with distinct spines arranged in almost single longitudinal row. Hind femur with fine dorsal protuberances. Hind basitarsus 0.7 times as long as second-fifth segments combined.

Metasoma. First tergite rather short and wide. Second tergite with narrow apical lenticular area delineated by rather shallow furrows. Second suture deep and almost straight. Fifth tergite rather distinctly enlarged, longer than previous tergite, covered following apical segments.

\section{Diagnosis}

This subgenus differs from nominative subgenus Rhaconotus $\mathrm{s}$. str. by the recurrent vein (m-cu) of fore wing weakly antefurcal (vs always postfurcal), the propodeum with distinct lateral tubercles (vs without or sometimes with small tubercles), and the apical segments of antenna contrastingly pale (vs always dark and the same colour as previous segments).

\section{Composition}

Rhaconotus (Pararhacon) haeselbarthi Belokobylskij, 2005.

\section{Hosts}

Unknown.

\section{Distribution}

Afrotropical region.

Rhaconotus (Pararhacon) haeselbarthi Belokobylskij, 2005

Figs $47-48$

Rhaconotus (Pararhacon) haeselbarthi Belokobylskij 2005: 208.

Rhaconotus (Pararhacon) haeselbarthi - Yu at al. 2016.

\section{Material examined}

\section{Holotype}

SOUTH AFRICA • +; “Mariepskop, Pilgrim's Rest dist., Tvl. [Transvaal], 14.iv.1964, E. Haeselbarth”; SSMG.

\section{Description}

Female

MeAsurements. Body length $3.2 \mathrm{~mm}$; fore wing length $2.0 \mathrm{~mm}$. 


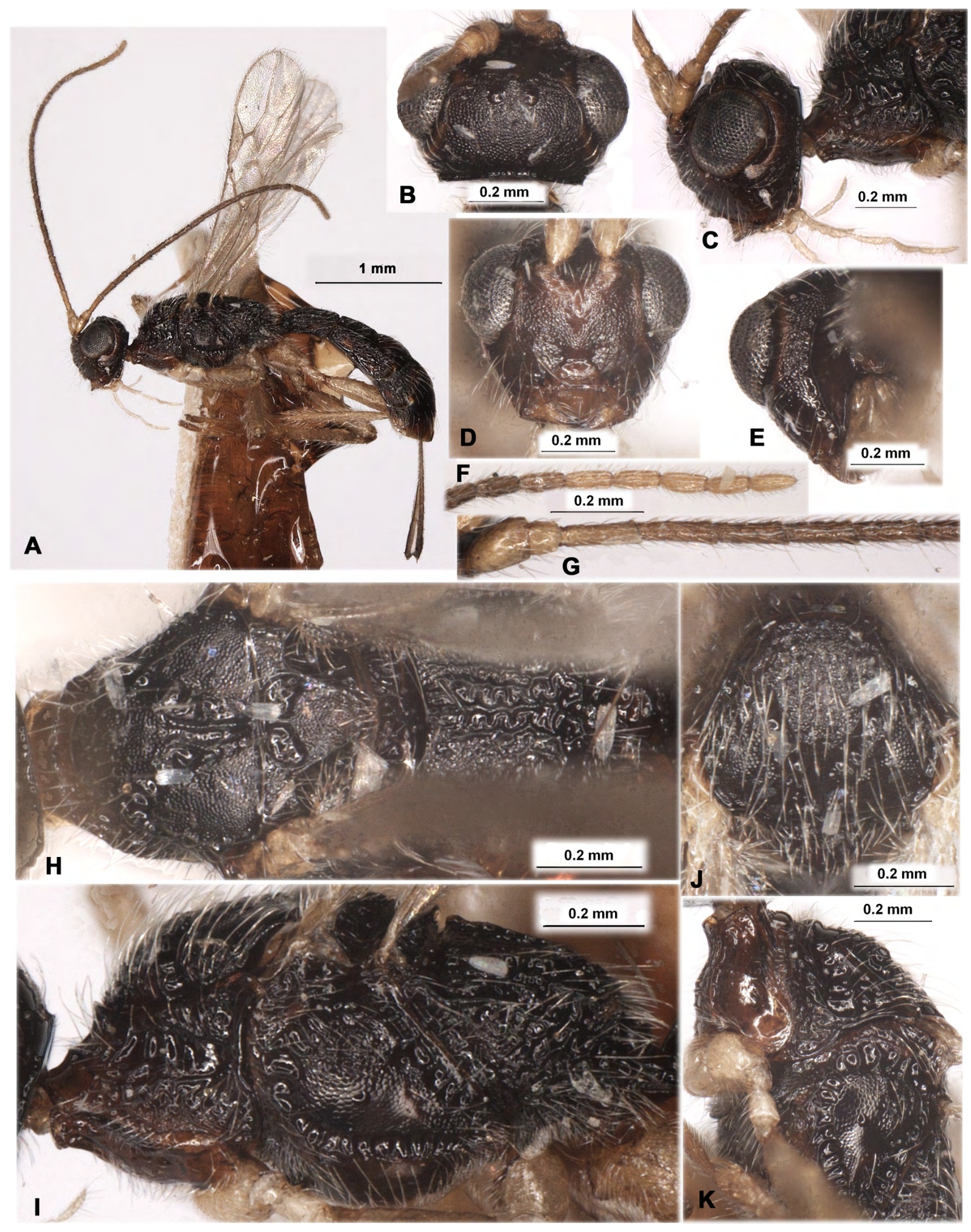

Fig. 47. Rhaconotus (Pararhacon) haeselbarthi Belokobylskij, 2005, + , holotype, SSMG. A. Habitus, lateral view. B. Head, dorsal view. C. Head, lateral view. D. Head, front view. E. Head, posterior view. F. Apical segments of antenna. G. Basal segments of antenna. H. Mesosoma, dorsal view. I. Mesosoma, lateral view. J. Mesoscutum. K. Pro-and mesopleura, lateral view. 
BELOKOBYLSKIJ S.A. \& ZALDÍVAR-RIVERÓN A., Reclassification of the Rhaconotini
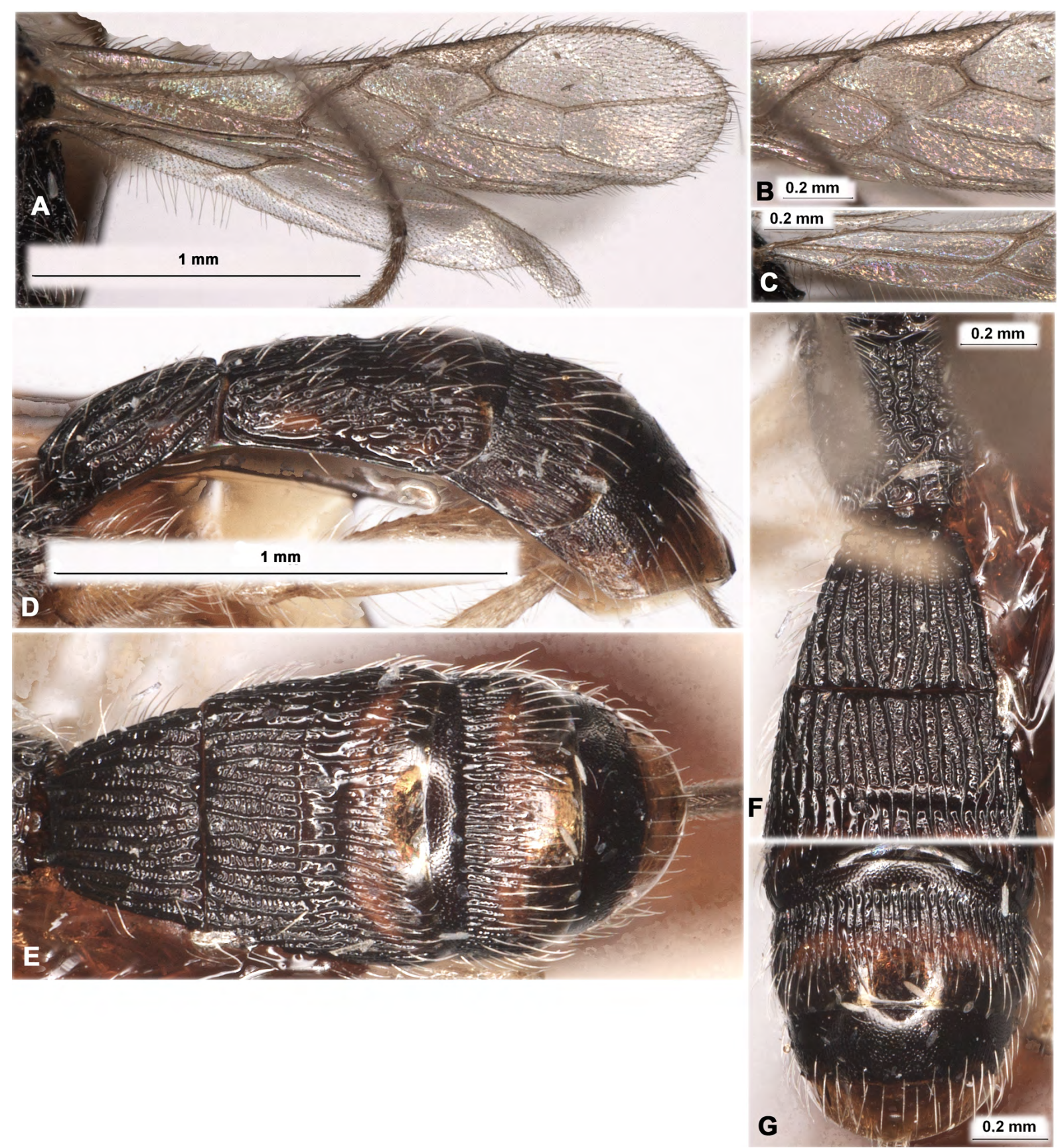

Fig. 48. Rhaconotus (Pararhacon) haeselbarthi Belokobylskij, 2005, $q$, holotype, SSMG. A. Wings. B. Median part of fore wing. C. Basal part of hind wing. D. Metasoma, lateral view. E. Metasoma, dorsal view. F. Propodeum and first to second metasomal tergites, dorsal view. G. Apex of metasoma, dorsal view. 
HEAD. Width 1.5 times its median length, 1.3 times width of mesoscutum (dorsal view). Head behind eyes (dorsal view) distinctly and almost linearly narrowed. Transverse diameter of eye 2.4 times length of temple. Ocelli small, arranged in triangle with base 1.2 times its sides; POL 1.2 times OD, 0.5 times OOL. Eye very weakly emarginated opposite antennal sockets, 1.3 times as high as broad. Malar space 0.65 times height of eye, 1.6 times basal width of mandible. Face width almost equal to height of eye and equal to height of face and clypeus combined. Upper margin of clypeus situated below line on lower level of eyes. Hypoclypeal depression width 0.6 times distance from edge of depression to eye, 0.35 times width of face. Vertex distinctly convex. Head below eyes (front view) distinctly and almost linearly narrowed. Antennae rather slender, filiform, 25-segmented, 0.9 times as long as body. Scapus 1.7 times as long as its maximum width. First flagellar segment 3.3 times as long as its apical width, 1.1 times as long as second segment. Penultimate segment 2.4 times as long as its width, 0.6 times as long as first segment, 0.85 times as long as apical segment.

Mesosoma. Length 1.9 times its height. Pronotal carina distinct, medial distances from carina to both sides of pronotum subequal. Mesoscutum 0.9 times as long as maximum width. Notauli entirely deep, rather wide, coarsely crenulate. Prescutellar depression deep, rather long, distinctly and linearly curved postero-laterally, distinctly rugulose, 0.3 times as long as the weakly convex scutellum. Sternaulus (precoxal furrow) rather deep, wide, weakly curved, distinctly and densely crenulate. Prepectal carina without widened lobes opposite fore coxae. Subalar depression rather shallow, more or less narrow, distinctly crenulate and almost smooth between crenulae. Metapleural lobe long, rather narrow, rounded apically, with dense pubescence posteriorly. Mesopleural suture entirely and coarsely crenulate.

Wings. Fore wing 3.8 times its maximum width. Metacarpus (1-R1) 1.2 times as long as pterostigma. First radial abscissa (r) forming very obtuse angle with second abscissa (3-SR). Second radial abscissa (3-SR) 3.3 times as long as first abscissa (r), 0.4 times as long as straight third abscissa (SR1), 1.15 times as long as first radiomedial vein (2-SR). Second radiomedial (submarginal) cell not widened distally, 2.6 times as long as wide, 1.6 times as long as brachial (subdiscal) cell. First medial abscissa $(1-\mathrm{SR}+\mathrm{M})$ weakly sinuate. Discoidal (discal) cell 2.3 times as long as its maximum width. Mediocubital vein $(\mathrm{M}+\mathrm{CU} 1)$ almost straight. Distance between nervulus (cu-a) and basal (1-M) vein almost equal to nervulus (cu-a) length. Brachial (subdiscal) cell narrow, almost perpendicularly closed distinctly before level of recurrent vein (m-cu); posterior bulla present; posterior abscissa of anal vein (2-1A) (behind brachial vein (CU1b)) indistinct. Hind wing 5.4 times as long as wide. First costal abscissa $(\mathrm{C}+\mathrm{SC}+\mathrm{R})$ 0.8 times as long as second abscissa (1-SC+R). First abscissa of mediocubital vein $(\mathrm{M}+\mathrm{CU}) 0.7$ times as long as second abscissa (1-M). Recurrent vein (m-cu) fine, unsclerotised, curved, weakly antefurcal.

Legs. Hind coxa 1.5 times as long as wide (with tubercle). Hind femur with fine dorsal protuberance, length of femur 3.8 times maximum width. Hind tarsus 0.9 times as long as hind tibia. Hind basitarsus 0.8 times combined length of second-fifth segments. Second tarsal segment 0.4 times as long as basitarsus, 2.0 times as long as fourth segment, 1.3 times as long as fifth segment (without pretarsus).

Metasoma. Length 1.1 times as long as head and mesosoma combined. First tergite with distinct but short subbasal lateral processes. Maximum width of first tergite 3.3 times its basal width; its length almost equal to apical width, 1.4 times length of propodeum. Second tergite with shallow and weakly curved subposterior transverse furrow indistinctly separated narrow apical area; median length of apical area about 0.4 times length of remaining part of tergite. Median length of second tergite 0.55 times its basal width, 1.35 times length of third tergite. Second suture rather wide, almost straight. Fifth tergite weakly convex in posterior margin, without median emargination and postero-ventral lobes. Fifth tergite 1.4 times as long as fourth tergite, 1.7 times as long as third tergite. Ovipositor sheath 0.55 times as long as metasoma, 0.8 times as long as mesosoma, 0.45 times as long as fore wing. 
SCULPTURE AND PUBESCENCE. Vertex entirely densely granulate, without striae or rugae; frons coarsely and irregularly densely rugose, with granulation medio-posteriorly; face densely and rather distinctly granulate, finely granulate to almost smooth medially; temple densely granulate with rugose posteriorly, below finely coriaceous to smooth. Mesoscutum entirely densely and distinctly granulate, with distinct rugosity on median lobe and laterally, with long coarse striae in medio-posterior half of mesoscutum. Scutellum densely and distinctly granulate. Mesopleuron densely and coarsely granulate-rugose, finely and widely coriaceous medially, smooth below sternaulus. Metapleuron coarsely densely rugosereticulate. Propodeum with distinctly delineated and densely granulate-rugose basolateral areas, with three longitudinal median carinae in basal half, coarsely areolate in posterior half. Hind coxa rather densely and distinctly granulate in upper half, rugulose medially and smooth below. Hind femur obliquely striate in upper half and smooth in lower half. First tergite with distinct, complete and subparallel dorsal carinae, distinctly, sparsely and coarsely striate and with dense and fine rugulosity between striae. Second tergite entirely coarsely and linearly striate with very dense and fine rugulosity between striae. Third tergite in basal 0.8 and fourth tergite in basal 0.5 distinctly and rather densely linearly striate and with additional fine or very fine reticulation, smooth apically. Fifth tergite densely and finely areolatecoriaceous in basal half and smooth in apical half. Second-fourth tergites laterally entirely and rather densely striate with granulation. Vertex entirely with long, sparse and semi-erect pale setae. Mesoscutum with rather dense, long and semi-erect setae arranged on all median lobe, along notauli and in single row laterally. Mesopleuron medially widely glabrous. Hind tibia dorsally with long, dense and semi-erect pale setae; length of these setae 1.0-1.2 times maximum width of tibia.

CoLour. Head reddish brown to dark reddish brown, black dorsally, with narrow reddish ring along eye. Mesosoma black, prothorax reddish brown to dark reddish brown. Metasoma reddish brown, almost black basally. Two basal segments of antenna yellow, flagellum mostly reddish brown to dark reddish brown, six apical segments brownish yellow. Palps yellow. Legs brownish yellow, tarsi faintly darker, hind tibia basally brown. Ovipositor sheath brownish yellow in basal 0.6 and brown in apical 0.4. Fore wing evenly faintly infuscate. Pterostigma yellowish brown, pale basally.

\section{Male}

Unknown.

\section{Distribution}

Republic of South Africa.

Genus Rhacontsira Belokobylskij, 1998

Rhacontsira Belokobylskij, 1998b: 2.

Rhacontsira - Belokobylskij et al. 2004: 89. — Belokobylskij \& Maeto 2006: 739; 2009: 453. - Yu et al. 2016. - Jasso-Martínez et al. 2019: 165.

\section{Type species}

Ontsira heterospiloides Belokobylskij, 1988

\section{Description (Figs 49-57)}

HEAD. Head transverse, not depressed. Ocelli arranged in obtuse triangle with base usually larger than their sides, but rarely in almostequilateral triangle. Frons not concave. Eyes glabrous. Occipital carina complete, lower often fused with hypostomal carina upper base of mandible. Malar suture usually absent, but sometimes present and and shallow or very shallow. Clypeal suture distinct and almost completre. Clypeus with distinct flange along lower margin. Hypoclypeal depression rather small, oval or round. Postgenal bridge present but very narrow. Palps rather long; maxillary palps 6-segmented, labial palps 
4-segmented. Scapus of antenna rather thick and short, without apical lobe and basal constriction. Pedicel relatively enlarged. Flagellum slender; first flagellar segment sometimes shorter than second segment, but usually subequal or weakly longer than it.

Mesosoma. Neck of prothorax short; pronotal keel usually distinct, but not connected with anterior margin of mesoscutum; dorsoposterior propleural lobe distinct. Mesonotum usually highly and almost vertically elevated above pronotum. Median lobe of mesoscutum without anterolateral corners and usually without medial longitudinal furrow. Notauli complete, sculptured, deep entirely or sometimes in anterior half only. Prescutellar depression relatively long, sculptured or smooth, with median carina. Scutellum weakly convex, without lateral carinae. Metanotum with short and pointed or obtuse median tooth (lateral view). Mesopleural pit distinct. Sternaulus (precoxal furrow) shallow, usually long, almost straight or sunuate, mostly crenulate. Prepectal carina distinct and complete. Propodeum with basolateral areas and usually with areola deliniated by distinct carinae; lateral tubercles and propodeal bridge absent. Propodeal spiracles small and round.

Wings. Pterostigma of fore wing rather wide. Radial vein (r) arising from or behind middle of pterostigma. Radial (marginal) cell not shortened. Both radiomedial veins (2-SR, r-m) present. Recurrent vein (mcu) antefurcal, rarely almost interstitial. Nervulus (cu-a) postfurcal. Discoidal (discal) cell petiolate, petiole (1-SR) short. Parallel vein (CU1a) not interstitial, arising from anterior third or almost middle of distal margin of brachial (subdiscal) cell. Brachial (subdiscal) cell narrow, closed distally behind recurrent vein $(\mathrm{m}-\mathrm{cu})$. Transverse anal veins $(2 \mathrm{~A}$, a) absent. Longitudinal anal vein $(2-1 \mathrm{~A})$ with short abscissa behind brachial vein (CU1b). In hind wing, radial (marginal) cell almost parallel-sided, without additional transverse vein (r). Medial (basal) cell rather narrow or wide distally, $0.4-0.5$ times as long as wing. Submedial (subbasal) cell short; first abscissa of mediocubital vein (M+CU) 0.5-0.7 times as long as second abscissa (1-M). Recurrent vein (m-cu) present, but usually finely sclerotised.

Legs. Fore and middle tibiae with numerous and small relatively dispersed spines. Hind coxa with distinct basoventral corner and tubercle. All femora with lower dorsal protuberances. Hind femur widened. Hind basitarsus $0.6-0.8$ times as long as second-fifth segments combined.

Metasoma. Metasoma with at least six visible tergites, following tergites shortly protruding behind sixth one. First tergite usually rather short and wide; dorsope distinct; present small and narrow basolateral lobes; spiracular tubercles indistinct or small, spiracles placed in basal third of tergite. Acrosternite of first segment about $0.20-0.25$ times as long as first tergite, its apical margin situated distinctly before spiracles. Second suture distinct and straight. Second tergite with almost straight, shallow of rather deep transverse furrow in apical third separating apical sublenticular area. Second-sixth tergites with separate laterotergites. Sometimes fourth or fifth segment at least weakly largened. Ovipositor shorter or longer than metasoma.

\section{Diagnosis}

Rhacontsira is morphologically similar to Rhaconotinus, but differs from it by having the recurrent vein (m-cu) of fore wing antefurcal to the first radiomedial vein (2-SR) (vs always postfurcal); the parallel vein (CU1a) not interstitial and arising from the middle or anterior third of the vein (3-CU1) closed brachial (subdiscal) cell distally (vs predominantly interstitial); metasoma behind third tergite mainly or entirely smooth (vs posterior tergites often sculptured at least basally); the first flagellar segment of antenna shorter, almost equal or weakly longer than second segment (vs always longer than second segment).

\section{Composition}

Rhacontsira haeselbarthi sp. nov. (AF); Rh. heterospiloides (Belokobylskij, 1988) (PA); Rh. insulicola Belokobylskij \& Maeto, 2006 (PA); Rh. mozambiquensis sp. nov. (AF); Rh. nana Belokobylskij, 1998 
(OR); Rh. saigonensis sp. nov. (OR); Rh. sculpturator Belokobylskij, 1998 (OR); Rh. toamasina sp. nov. (AF); Rh. toyota Belokobylskij \& Maeto, 2006 (PA); Rh. variegata Belokobylskij, Iqbal \& Austin, 2004 (AU); Rh. yamagishii Belokobylskij \& Maeto, 2006 (PA).

\section{Hosts}

Unknown.

\section{Distribution}

Afrotropical, Australasian Oriental and Palaearctic regions.

\section{Key to species of Rhacontsira}

1. Vertex entirely finely coriaceous (Fig. 56C). Apical segments of antenna white, contrastingly lighter than previous segments (Figs 56A, E). Second metasomal tergite with very short and smooth basal area (Fig. 57B). Body length $5.0 \mathrm{~mm}$. Madagascar Rh. toamasina sp. nov.

- Vertex at least partly striate, rarely mainly smooth, never coriaceous (Figs 49C, 51B, 52C, G, 54C). Apical segments of antenna dark, same colour as previous segments (Figs 51F, 52F, 54E). Second metasomal tergite without distinct basal area (Figs 50C, 51H, 53B, 55B)

2. Head (dorsal view) less distinctly and convex-roundly narrowed behind eyes (Figs 49C, 51B). Temple long, transverse diameter of eye 1.5-1.6 times as long as temple (Figs 49C, 51B). First flagellar segment $0.80-0.85$ times as long as second segment (Figs 49D, 51E) …......................... 3

- Head (dorsal view) rather strongly and weakly-roundly narrowed behind eyes (Figs 52C, G, 54C). Temple short, transverse diameter of eye 1.8-2.2 times as long as temple (Figs 52C, G, 54C). First flagellar segment almost as long as or only a little shorter than second segment (Figs 52E, 54D) ....

3. Malar space 0.3 times height of eye and 0.5-0.6 times basal width of mandible (Fig. 49B). Pronotal carina situated submedially on pronotum (dorsal view) (Fig. 49E). Second radial abscissa (3-SR) of fore wing 0.80-0.85 times as long as first radiomedial vein (2-SR) (Fig. 50A). Hind femur 3.2 times as long as wide (Fig. 49G). Ovipositor sheath 2.0 times as long as mesosoma and 0.75 times as long as fore wing (Fig. 49A). Body length 2.1-2.2 mm. Eastern Palaearctic: Japan, Russia

Rh. heterospiloides (Belokobylskij, 1988)

- Malar space 0.45 times height of eye and 0.9 times basal width of mandible (Fig. 51D). Pronotal carina situated on pronotum more closely to mesoscutum (dorsal view). Second radial abscissa (3SR) of fore wing 1.1 times as long as first radiomedial vein (2-SR) (Fig. 51I). Hind femur 3.7 times as long as wide. Ovipositor sheath 1.3 times as long as mesosoma and 0.55 times as long as fore wing (Fig. 51A). Body length $1.8 \mathrm{~mm}$. Afrotropics: South Africa

Rh. haeselbarthi sp. nov.

4. Frons entirely distinctly and densely striate (Figs 52B, C, G). Mesosoma 1.4-1.6 times as long as high (Fig. 52H). Metapleural flange posteriorly with very dense pubescence (Fig. 52H). Second radiomedial (submarginal) cell 1.2-1.4 times as long as brachial (subdiscal) cell. Second radial abscissa (3-SR) 0.7-0.9 times as long as first radiomedial vein (2-SR) (Figs 52K, 53A). First tergite shorter or almost as long as its apical width (Fig. 53B). Areola of propodeum almost indistinct (Fig. 52I)

- Frons at least partly or almost entirely rugulose-reticulate with punctation or granulation, often medially or laterally smooth (Figs 54B-C, F). Mesosoma 1.7-1.9 times as long as high (Fig. 54G). Metapleural flange posteriorly with sparce pubescence (Fig. 54G). Second radiomedial (submarginal) cell 1.4-1.8 times as long as brachial (subdiscal) cell. Second radial abscissa (3-SR) 0.9-1.2 times as long as first radiomedial vein (2-SR) (Fig. 55A). First tergite 1.1-1.5 times as long as its apical width (Fig. 55B). Areola of propodeum distinctly delineated (Fig. 54F) 
5. First flagellar segment thick, 3.8-4.3 times as long as its apical width and not longer than second segment. Ocelli arranged in almost equilateral triangle. Frons entirely distinctly and densely oblique striae (Figs.). First metasomal tergite almost as long as its maximum width. Body length 1.5-3.0 mm. Eastern Palaearctic: Japan

Rh. insulicola Belokobylskij \& Maeto, 2006

- First flagellar segment slender, 5.2 times as long as its apical width and 1.1 times as long as second segment (Fig. 52E). Ocelli arranged in triangle with base 1.3 times its sides (Fig. 52C). Frons rugose-granulate and with curved longitudinal carinae laterally (Fig. 52C). First metasomal tergite 0.8 times as long as its maximum width (Fig. 53B). Body length 3.3-3.6 mm. Afrotropics: Mozambique

Rh. mozambiquensis sp. nov.

6. Frons mostly smooth, only narrowly anteriorly with fine sculpture. Prescutellar depression smooth or almost smooth. Hind femur 3.6-4.0 times as long as its width

- Frons entirely or at least laterally and widely anteriorly densely striate or rugulose-reticulate with punctation (Figs 54B, C). Prescutellar depression distinctly sculptured (Fig. 54F). Hind femur 3.23.6 times as long as its width (Fig. $54 \mathrm{H}$ )

7. Ovipositor sheath shorter, as long as metasoma, 1.3 times as long as mesosoma, 0.5 times as long as fore wing. Length of first metasomal tergite of female 1.5 times its apical width. Body length 1.7 $\mathrm{mm}$. Vietnam

Rh. nana Belokobylskij, 1998

- Ovipositor sheath longer, 1.3-1.5 times as long as metasoma, twice longer than mesosoma, 0.800.85 times as long as fore wing. Length of first metasomal tergite of female 1.2-1.3 times its apical width. Body length 1.6-2.0 mm. Japan Rh. yamagishii Belokobylskij \& Maeto, 2006

8. Body entirely or mainly brownish yellow (Fig. 54A). Fore wing hyaline (Fig. 55A). Antenna 18-20-segmented (Fig. 54A). Body length $1.7 \mathrm{~mm}$. Vietnam Rh. saigonensis $\mathrm{sp}$. nov.

- Body entirely or mainly black, partly with reddish tint, rarely head below and mesonotum partly lightly reddish brown. Fore wing infuscate. Antenna 22-23-segmented. Body length $2.1-2.9 \mathrm{~mm}$

9. Temple almost entirely rugulose-reticulate. Parallel vein (CU1a) of fore wing arising almost from middle of distal margin of brachial (subdiscal) cell. Pterostigma entirely dark brown. Body length $2.9 \mathrm{~mm}$. Vietnam Rh. sculpturator Belokobylskij, 1998

- Temple entirely smooth. Parallel vein (CU1a) of fore wing arising distinctly before middle of distal margin of brachial (subdiscal) cell. Pterostigma brown, yellow or pale brown basally and sometimes apically

10. Vertex mainly finely striate-coriaceous, only coriaceous laterally, almost smooth in small anterolateral areas. Frons without longitudinal median carina. First flagellar segment 4.6 times as long as its apical width. Pronotal keel distinct. Mesopleuron behind deep posterior part of sternaulus till middle coxa with shallow, narrow and longitudinally striate furrow. Second radiomedial (submarginal) cell 2.6 times as long as maximum width. Second segment of hind tarsus 0.3 times as long as basitarsus. Body length $2.4 \mathrm{~mm}$. Japan Rh. toyota Belokobylskij \& Maeto, 2006

- Vertex finely transverse striate in anterior $0.5-0.7$, smooth posteriorly. Frons with longitudinal median carina. First flagellar segment 5.0-5.5 times as long as its apical width. Pronotal keel very fine. Mesopleuron behind deep posterior part of sternaulus without furrow. Second radiomedial (submarginal) cell 3.5 times as long as maximum width. Second segment of hind tarsus $0.45-0.50$ times as long as basitarsus. Body length $2.1-2.8 \mathrm{~mm}$. Australia 
BELOKOBYLSKIJ S.A. \& ZALDÍVAR-RIVERÓN A., Reclassification of the Rhaconotini

Rhacontsira heterospiloides (Belokobylskij, 1988)

Figs 49-50

Ontsira heterospiloides Belokobylskij, 1988b: 627.

Rhacontsira heterospiloides - Belokobylskij 1998a: 4. — Belokobylskij \& Maeto 2006: 739; 2009: 453. - Yu et al. 2016.

\section{Material examined}

Holotype

RUSSIA • P; "Primorskiy krai [Territory], Brovnichi, shir.[okolistvennyi] les u reki [broadleaf forest near river], Belokobylskij [leg.], 18 VII 1984", "Holotypus Ontsira heterospiloides Belokobylskij"; ZISP.

\section{Description}

\section{Female}

Measurements. Body length $2.2 \mathrm{~mm}$; fore wing length $1.9 \mathrm{~mm}$.

HEAD. Width 1.5 times its median length, 1.2 times width of mesoscutum. Head behind eyes (dorsal view) distinctly and roundly narrowed. Transverse diameter of eye 1.6 times length of temple (dorsal view). Ocelli medium-sized, arranged in triangle with base 1.2 times its sides; POL 1.3 times OD, 0.5 times OOL. Eye glabrous, weakly emarginated opposite antennal sockets, 1.2 times as high as broad. Malar space 0.3 times height of eye, 0.55 times basal width of mandible. Face width almost equal to height of eye and 1.1 times height of face and clypeus combined. Hypoclypeal depression round, its width almost equal to distance from edge of depression to eye, 0.5 times width of face. Occipital carina not fused ventrally with hypostomal carina being obliterated shortly upper base of mandible. Vertex distinctly convex. Head below eyes distinctly and weakly-roundly narrowed (front view). Antennae slender, filiform, 18-segmented, as long as body. Scapus 1.8 times as long as its maximum width and pedicel. First flagellar segment 4.5 times as long as its apical width, 0.9 times as long as second segment. Penultimate segment 4.0 times as long as wide, 0.9 times as long as first segment, almost equal to apical segment.

Mesosoma. Length 1.8 times its height. Pronotal carina distinct, distances from carina to both sides of pronotum subequal. Mesoscutum 0.9 times as long as wide. Notauli deep anteriorly and weakly shallow posteriorly, rather wide, crenulate with fine rugulosity. Prescutellar depression deep, very weakly and linearly oblique postero-laterally, with high median and two lateral carinae, almost smooth, 0.45 times as long as the convex scutellum. Sternaulus (precoxal furrow) distinctly and densely rugose-reticulate. Subalar depression sparsely and coarsely crenulate and with fine rugulosity between striae. Metanotum with short tooth directed backward. Metapleural lobe rather long, narrow, rounded apically, with relatively dense pubescence. Propodeum (lateral view) distinctly convex.

WINGs. Fore wing 3.0 times its maximum width. Pterostigma 4.2 times as long as its maximum width. Metacarpus (1-R1) 1.2 times as long as pterostigma. Radial vein (r) arising from middle of pterostigma. First radial abscissa (r) forming obtuse angle with second abscissa (3-SR). Second radial abscissa (3SR) 1.8 times as long as first abscissa (r), 0.3 times as long as the straight third abscissa (SR1), 0.8 times as long as first radiomedial vein (2-SR). Second radiomedial (submarginal) cell weakly narrowed distally, 2.7 times as long as wide, 1.5 times as long as the narrow brachial (subdiscal) cell. First medial abscissa (1-SR+M) almost straight. Recurrent vein (m-cu) 4.5 times as long as second medial abscissa $(2-\mathrm{SR}+\mathrm{M}), 0.6$ times as long as first radiomedial vein (2-SR). Mediocubital vein $(\mathrm{M}+\mathrm{CU} 1)$ almost straight. Nervulus (cu-a) postfurcal, distance between nervulus (cu-a) and basal (1-M) vein equal to 


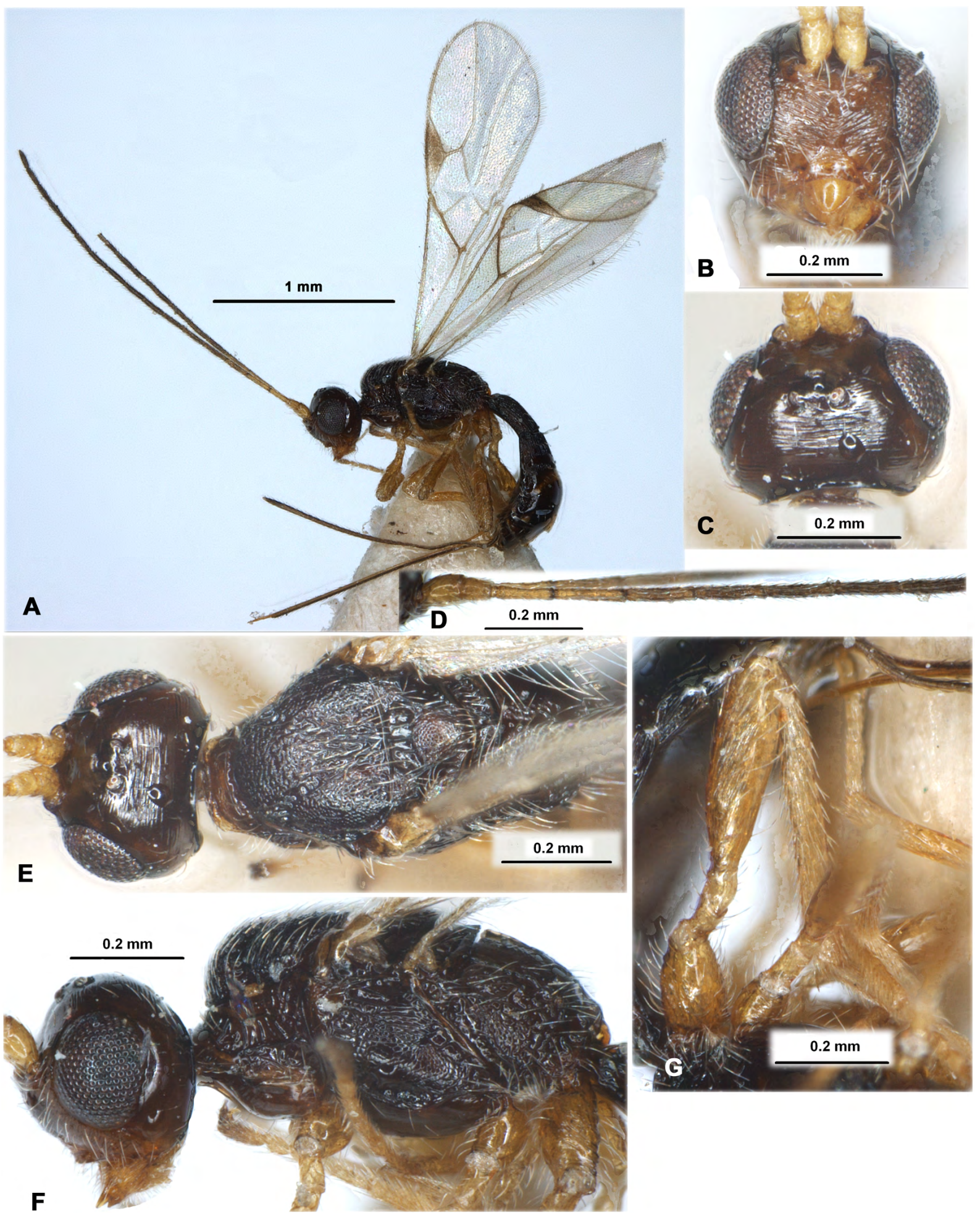

Fig. 49. Rhacontsira heterospiloides (Belokobylskij, 1988),, , holotype, ZISP. A. Habitus, lateral view. B. Head, front view. C. Head, dorsal view. D. Basal segments of antenna. E. Head and mesosoma, dorsal view. F. Head and mesosoma, lateral view. G. Hind leg, lateral view. 
BELOKOBYLSKIJ S.A. \& ZALDÍVAR-RIVERÓN A., Reclassification of the Rhaconotini
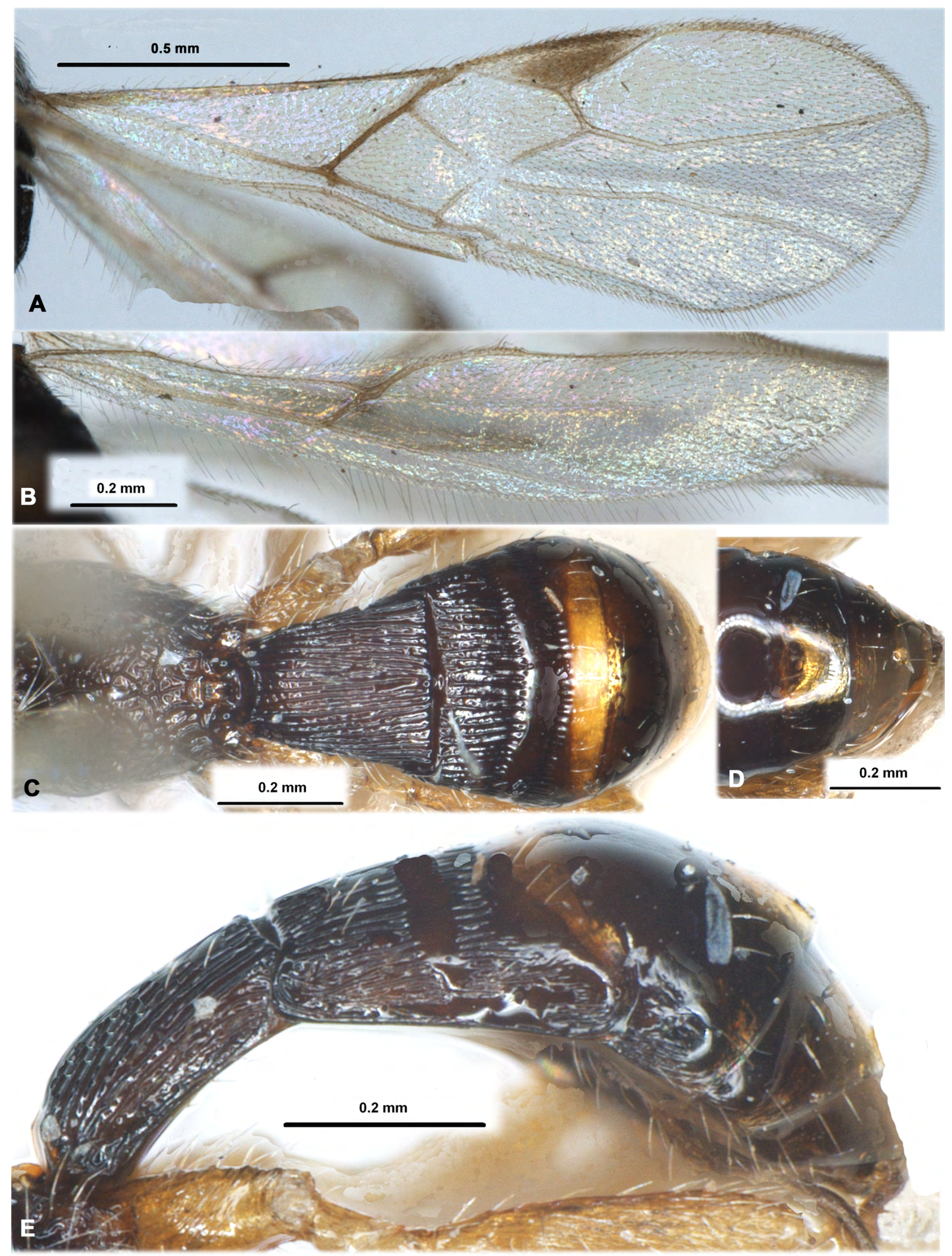

Fig. 50. Rhacontsira heterospiloides (Belokobylskij, 1988),, , holotype, ZISP. A. Fore wing. B. Hind wing, C. Propodeum and metasoma, dorsal view. D. Apex of metasoma, dorsal view. E. Metasoma, lateral view. 
nervulus (cu-a) length. Hind wing 5.4 times as long as wide. First abscissa of mediocubital vein $(\mathrm{M}+\mathrm{CU})$ 0.7 times as long as second abscissa (1-M). Recurrent vein (m-cu) antefurcal.

LeGs. Hind coxa 1.5 times as long as wide (with basoventral tubercle). Hind femur 3.2 times as long as its maximum width. Hind tarsus 0.9 times as long as hind tibia. Second tarsal segment 0.45 times as long as basitarsus, almost as long as fifth segment (without pretarsus).

Metasoma. Length 1.1 times as long as head and mesosoma combined. First tergite distinctly and almost linearly widened towards apex. Maximum width of first tergite 2.2 times its basal width; length 1.1 times apical width, 1.2 times length of propodeum. Second tergite with distinct shallow and transverse furrow in posterior third separating rather short transverse apical area. Length of second tergite 0.6 times its basal width, 1.7 times length of third tergite. Second suture rather deep, weakly curved and entirely crenulate. Fourth tergite enlarged, weakly convex in posterior margin, without median emargination and postero-ventral lobes, 1.5 times as long as third tergite, 1.7 times as long as fifth tergite. Ovipositor sheath 1.2 times as long as metasoma, about 2.0 times as long as mesosoma, 0.75 times as long as fore wing.

SCUlPTURE AND PUBESCENCE. Vertex distinctly and densely transverse striate, smooth laterally and posteriorly; frons finely transverse curvedly aciculate, partly smooth; face densely and distinctly obliquely striate, with very fine rugulosity between striae; temple smooth. Mesoscutum entirely densely granulate, median lobe additionally with small rugulosity, without rugosity along notauli, rugosereticulate in narrow area in its medio-posterior 0.3. Scutellum densely and rather finely granulate, granulate-coriaceous posteriorly. Mesopleuron widely reticulate-coriaceous. Metapleuron densely rugose-reticulate and partly with granulation. Basolateral areas of propodeum large, reticulate-rugulose, but finely coriaceous to almost smooth in their medio-anterior 0.3 , with distinct median carina in basal 0.3 of propodeum; remainder of propodeum reticulate-rugose. Hind coxa densely rugose with striation partly in dorsal half, smooth in ventral half. Hind femur densely obliquely striate in dorsal half and smooth or almost smooth in lower half. First tergite entirely, second mostly (except smooth apical area), third and fourth tergite narrow laterally distinctly and densely longitudinally striate, with fine or very fine reticulation between striae. Remainder of tergites smooth. Vertex with very sparse, short and semierect pale setae, glabrous in medio-anterior part. Mesoscutum entirely with rather dense, short and semierect setae. Mesopleuron glabrous on wide median area. Hind tibia dorsally with short, rather dense and semi-erect pale setae; length of these setae $0.3-0.5$ times maximum width of tibia.

CoLour. Body dark reddish brown, head paler ventrally, reddish brown to light reddish brown; third tergite of metasoma brownish yellow in transverse posterior half. Antenna dark brown to black, four basal segments yellow to brownish yellow. Palps yellow. Legs brownish yellow or light reddish brown, hind tibia pale basally. Ovipositor sheath black. Fore wing very faintly and evenly infuscate. Pterostigma brown, yellow in basal quarter and apically.

Male

Unknown.

\section{Distribution}

Japan (Honshu), Russia (S of Far East). 


\section{Rhacontsira haeselbarthi sp. nov. urn:1sid:zoobank.org:act:D2A46876-0B35-4BDE-B49F-FB7927B69864}

Fig. 51

\section{Etymology}

Named after Dr E. Haeselbarth, well-known German hymenopterist and collector of the holotype specimen.

\section{Material examined}

Holotype

REPUBLIC OF SOUTH AFRICA • +; "Entabeni Forest, Soutpansberg, Tvl. [Transvaal], 4-5000', 3.7.6.[19]64, E. Haeselbarth"; SSMG.

\section{Description}

\section{Female}

Measurements. Body length $1.8 \mathrm{~mm}$; fore wing length $1.7 \mathrm{~mm}$.

HEAD. Width 1.4 times its median length, 1.2 times width of mesoscutum. Head behind eyes (dorsal view) roundly narrowed. Transverse diameter of eye 1.6 times as long as temple (dorsal view). Ocelli small, arranged in triangle with base 1.2 times its sides. POL 1.5 times OD, 0.5 times OOL. Eye glabrous, very weakly emarginated opposite antennal sockets, 1.25 times as high as broad. Malar space 0.45 times height of eye and 0.9 times basal width of mandible. Face width 1.1 times height of eye and 1.3 times height of face and clypeus combined. Clypeal suture distinct and complete. Hypoclypeal depression round, its width almost equal to distance from edge of depression to eye, 0.4 times width of face. Occipital carina complete dorsally, fused below with hypostomal carina above base of mandible. Hypostomal flange distinct. Vertex distinctly convex. Head below eyes distinctly and roundly narrowed (front view). Antennae slender, filiform, 19-segmented, 1.3 times as long as body. Scapus 1.8 times as long as maximum width. First flagellar segment 4.5 times as long as its apical width, 0.85 times as long as second segment. Penultimate segment 4.0 times as long as width, as long as first flagellar and apical segments; the latter distinctly pointed.

Mesosoma. Mesosoma not depressed. Length almost twice its height. Pronotum with distinct pronotal carina situated more closely to mesoscutum, but not fused with it. Mesoscutum highly and almost perpendicularly raising above pronotum (lateral view), almost as long as maximum width. Median lobe of mesoscutum not protruding forward. Notauli deep anteriorly, shallow posteriorly, narrow, crenulategranulate. Prescutellar depression rather deep and long, weakly curved postero-laterally, with distinct median carina, finely rugulose, about 0.5 times as long as weakly convex scutellum. Subalar depression shallow, wide, rugose-granulate. Sternaulus (precoxal furrow) distinct, rather deep, finely crenulategranulate, connected with prepectal carina anteriorly, running along entire length of lower part of mesopleuron, weakly curved medially. Metanotum without dorsal tooth (lateral view). Metapleural lobe short, widened, rounded apically, with sparse pubescence.

WING. Fore wing 3.2 times as long as its maximum width. Pterostigma 4.5 times as long as wide. Metacarpus (1-R1) 1.3 times as long as pterostigma. Radial vein (r) arising almost from middle of pterostigma. First radial abscissa (r) forming obtuse angle with second abscissa (3-SR). Second radial abscissa (3-SR) 2.4 times first abscissa (r), 0.4 times the straight third abscissa (SR1), 1.1 times first radiomedial vein (2-SR). Second radiomedial (submarginal) cell not narrowed distally, about 3.0 times as long as maximum width, 1.4 times as long as the narrow brachial (subdiscal) cell. First medial abscissa $(1-\mathrm{SR}+\mathrm{M})$ weakly sinuate. Recurrent vein $(\mathrm{m}-\mathrm{cu})$ about 4.0 times as long as second medial abscissa, 0.6 times as long as first radiomedial vein (2-SR). Mediocubital vein (M+CU1) almost straight. Distance 

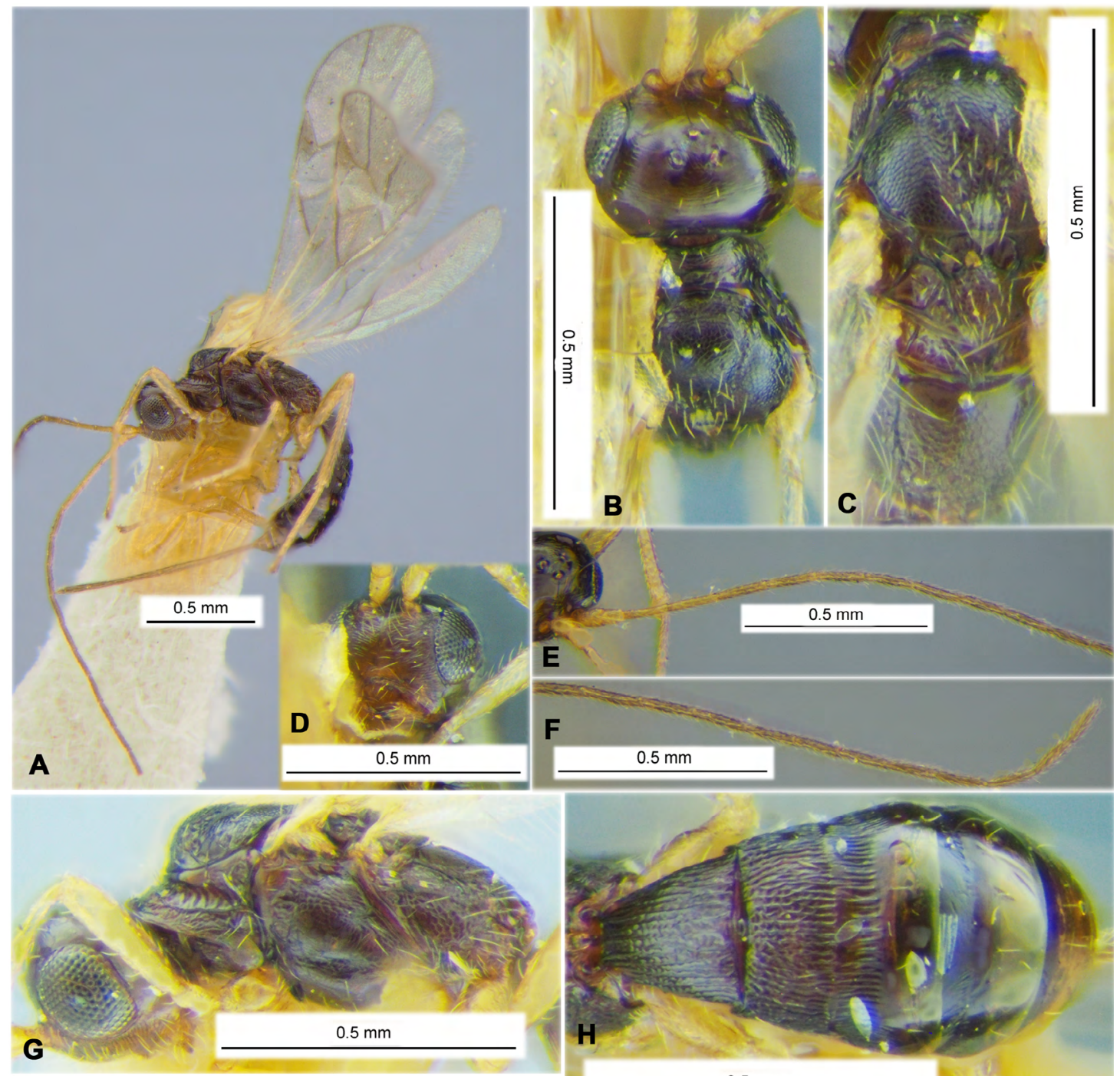

E
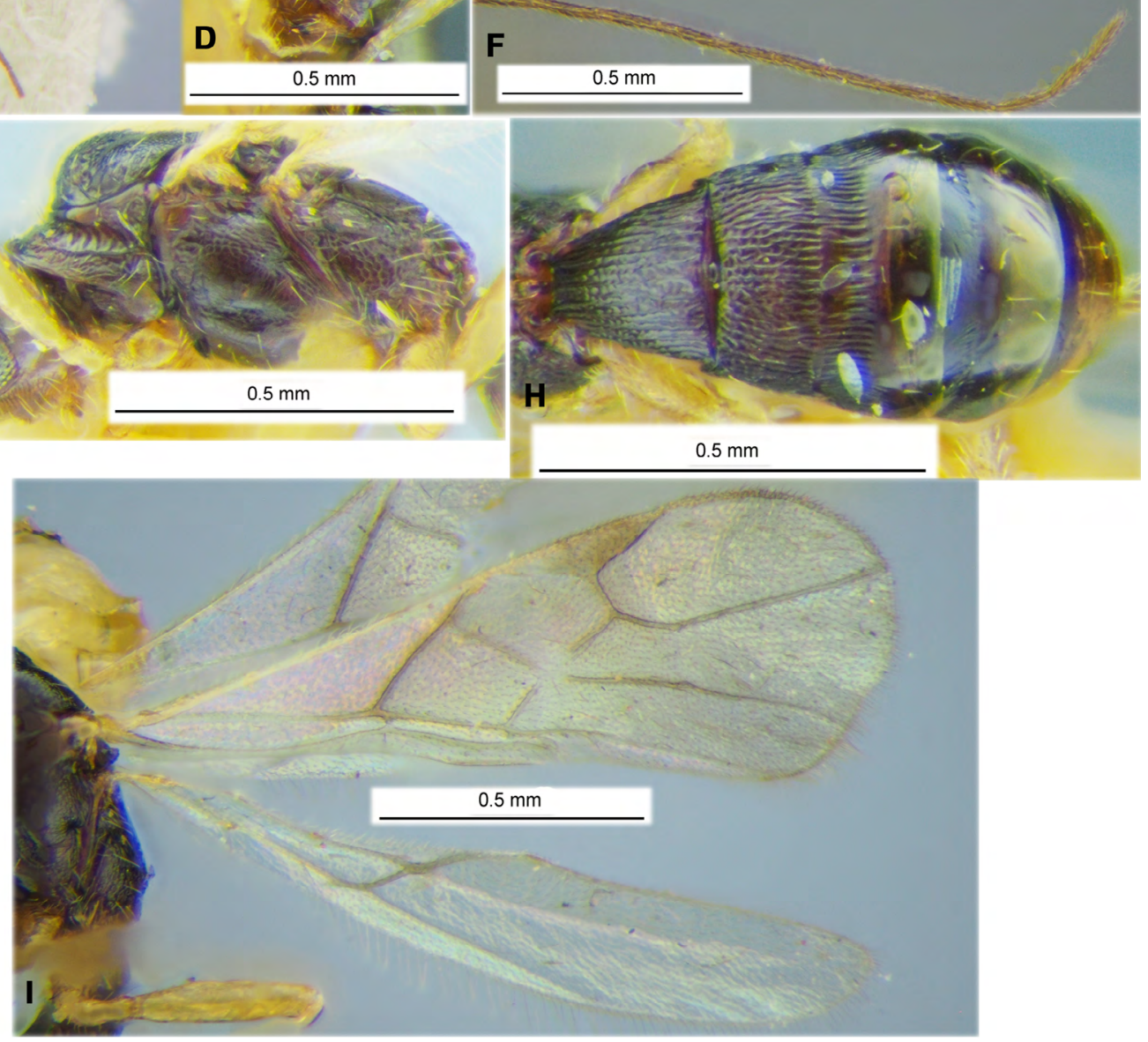

Fig. 51. Rhacontsira haeselbarthi sp. nov.,, , holotype, SSMG. A. Habitus, lateral view. B. Head and mesoscutum, front view. C. Mesosoma, dorsal view. D. Head, front view. E. Basal segments of antenna. F. Apical segments of antenna. G. Head and mesosoma, lateral view. H. Metasoma, dorsal view. I. Wings. 
from nervulus (cu-a) to basal (1-M) vein almost equal to nervulus (cu-a) length. Hind wing 5.6 times as long as wide. First costal abscissa $(\mathrm{C}+\mathrm{SC}+\mathrm{R}) 0.6$ times as long as second abscissa (1-SC+R). First abscissa of mediocubital vein $(\mathrm{M}+\mathrm{CU}) 0.7$ times as long as second abscissa (1-M). Recurrent vein (m$\mathrm{cu}$ ) weakly curved, strongly oblique, strongly antefurcal.

Legs. Hind coxa 1.6 times as long as wide (with basoventral tubercle). Hind femur 3.7 times as long as wide. Hind tarsus 0.9 times as long as hind tibia. Hind basitarsus 0.75 times as long as secondfifth segments combined; second tarsal segment 0.4 times as long as basitarsus, almost as long as fifth segment (without pretarsus).

Metasoma. Length almost as long as mesosoma and head combined. First tergite with distinct pointed and directed downwards basolateral processes, without spiracular tubercles, distinctly and linearly widened from base to apex. Maximum width of first tergite 2.5 times minimum width; length 1.2 times its apical width, 1.1 times length of propodeum. Second tergite with rather distinct shallow and transverse furrow in posterior quarter separating rather short transverse apical area. Length of second tergite 0.7 times its basal width, almost twice length of third tergite. Second suture rather deep, almost straight, weakly curved laterally. Fourth tergite enlarged, weakly convex in posterior margin, without median emargination and postero-ventral lobes, 1.7 times as long as third tergite, 1.3 times as long as fifth tergite. Ovipositor sheath 1.1 times as long as metasoma, 1.3 times as long as mesosoma, 0.55 times as long as fore wing.

SCULPTURE AND PUBESCENCE. Vertex very finely transversely striate, almost smooth in posterior third; frons mostly smooth, finely rugulose-coriaceous in anterior third; face distinctly densely striate, smooth medially and below; temple smooth. Mesoscutum entirely densely granulate, without rugosity along notauli, rugose on its small medio-posterior area. Scutellum densely granulate. Mesopleuron finely coriaceous, granulate-coriaceous upper, very finely coriaceous to smooth near sternauli. Metapleuron densely rugose-reticulate, finer sculptured in anterior third. Propodeum with rugulose-granulate at most part and almost smooth basally basolateral areas distinctly delineated by carinae; with distinct median carina in its basal 0.5 ; remainder of propodeum rugose-reticulate with granulation; areola indistinct. Hind coxa granulate, finely coriaceous below. Hind femur finely and densely striate, smooth below. First and second tergites densely longitudinally striate with dense ground reticulation between striae; apical area of second tergite finer and irregularly striate. Third tergite laterally in basal two-thirds and fourth tergite laterally in basal half densely striate, these tergites medially very shortly striate or rugulose basally. Remaining parts of tergites smooth. Vertex with very sparse short semi-erect setae. Mesoscutum with sparse, rather short and semi-erect setae arranged along notauli and marginally. Mesopleuron glabrous on wide median area.Hind tibia with short and dense semi-erect setae; length of these setae about half maximum width of hind tibia.

CoLour. Body black with reddish tint. Antenna mainly almost black, five basal segments yellow or light brown. Palpi yellow. Legs brownish yellow, coxae faintly infuscate. Ovipositor sheath brown, almost black apically. Fore wing faintly and evenly infuscate. Pterostigma brown, yellow in basal third.

\section{Male \\ Unknown.}

\section{Diagnosis}

This new species is morphologically very similar to the Eastern Palaearctic Rh. heterospiloides (Belokobylskij), but differs from the latter by having the malar space 0.45 times height of eye and 0.9 times basal width of mandible (vs 0.3 and 0.55 times, correspondingly), pronotal carina situated more closely to mesoscutum (vs situated submedially), second radial abscissa (3-SR) of fore wing 1.1 times 
as long as first radiomedial vein (2-SR) (vs only 0.8 times), hind femur 3.7 times as long as wide (vs 3.2 times), ovipositor sheath 1.3 times as long as mesosoma and 0.55 times as long as fore wing (vs about 2.0 and 0.75 times, correspondingly), and it is also distributed in the Afrotropical region (vs the Eastern Palaearctic ).

\section{Distribution}

Republic of South Africa.

Rhacontsira mozambiquensis sp. nov. urn:Isid:zoobank.org:act:93E97C3E-D8C9-4EE1-B153-9755937A34CD

Figs $52-53$

\section{Etymology}

Named after the country where the type material was collected, Mozambique.

\section{Material examined}

Holotype

MOZAMBIQUE • + ; "Museum Paris, Mozambique, Haut Sangadze, Inhafounè pres Canxixe, P. Lesne, 1928", "Juin"; MNHN.

\section{Paratype}

MOZAMBIQUE $\bullet 1$ \& (head partly damaged in right side); same collection data as for holotype; ZISP.

\section{Description}

Female

Measurements. Body length 3.3-3.6 mm; fore wing length 2.7-3.0 mm.

HeAD. Width 1.6-1.8 times its median length, 1.1-1.2 times maximum width of mesoscutum. Head behind eyes (dorsal view) strongly roundly narrowed. Transverse diameter of eye 2.4-2.5 times as long as temple (dorsal view). Ocelli medium-sized, arranged in triangle with base 1.2-1.3 times its sides. POL 1.5-1.7 times OD, 0.5-0.7 times OOL. Eye glabrous, 1.2 times as high as broad. Malar space about 0.4 times height of eye and 1.0-1.1 times basal width of mandible. Face width equal to height of eye and almost equal to height of face and clypeus combined. Malar suture absent. Clypeus rather high, with short lower flange. Clypeal suture distinct. Hypoclypeal depression round, its width 0.9 times distance from edge of depression to eye, 0.4 times width of face. Occipital carina coarse, complete dorsally, fused below with hypostomal carina above base of mandible. Hypostomal flange relatively wide. Head (front view) distinctly and almost linearly narrowed below eyes. Antennae slender, setiform, 29-segmented, 1.2 times as long as body. Scapus 1.5-1.7 times as long as maximum width. First flagellar segment 5.0-5.2 times as long as its apical width, about 1.1 times as long as second segment. Penultimate segment 5.5 times as long as width, as long as first flagellar and apical segments; the latter distinctly acuminate.

Mesosoma. Mesosoma not depressed, 1.4-1.6 times as long as its high. Pronotum with distinct pronotal carina situated more closely to mesoscutum, but not fused with it. Mesoscutum highly and almost perpendicularly elevated above pronotum. Median lobe of mesoscutum not protruding forward. Notauli complete, distinct anteriorly, shallow posteriorly, coarsely crenulate-rugose. Prescutellar depression deep, rather short, with high median and two-three lateral carinae, finely rugulose to smooth between carinae, $0.3-0.4$ times as long as very weakly convex scutellum. Metanotum with short, thick and subacuminate dorsal tooth (lateral view). Subalar depression shallow, narrow, rugose-striate. Sternaulus 


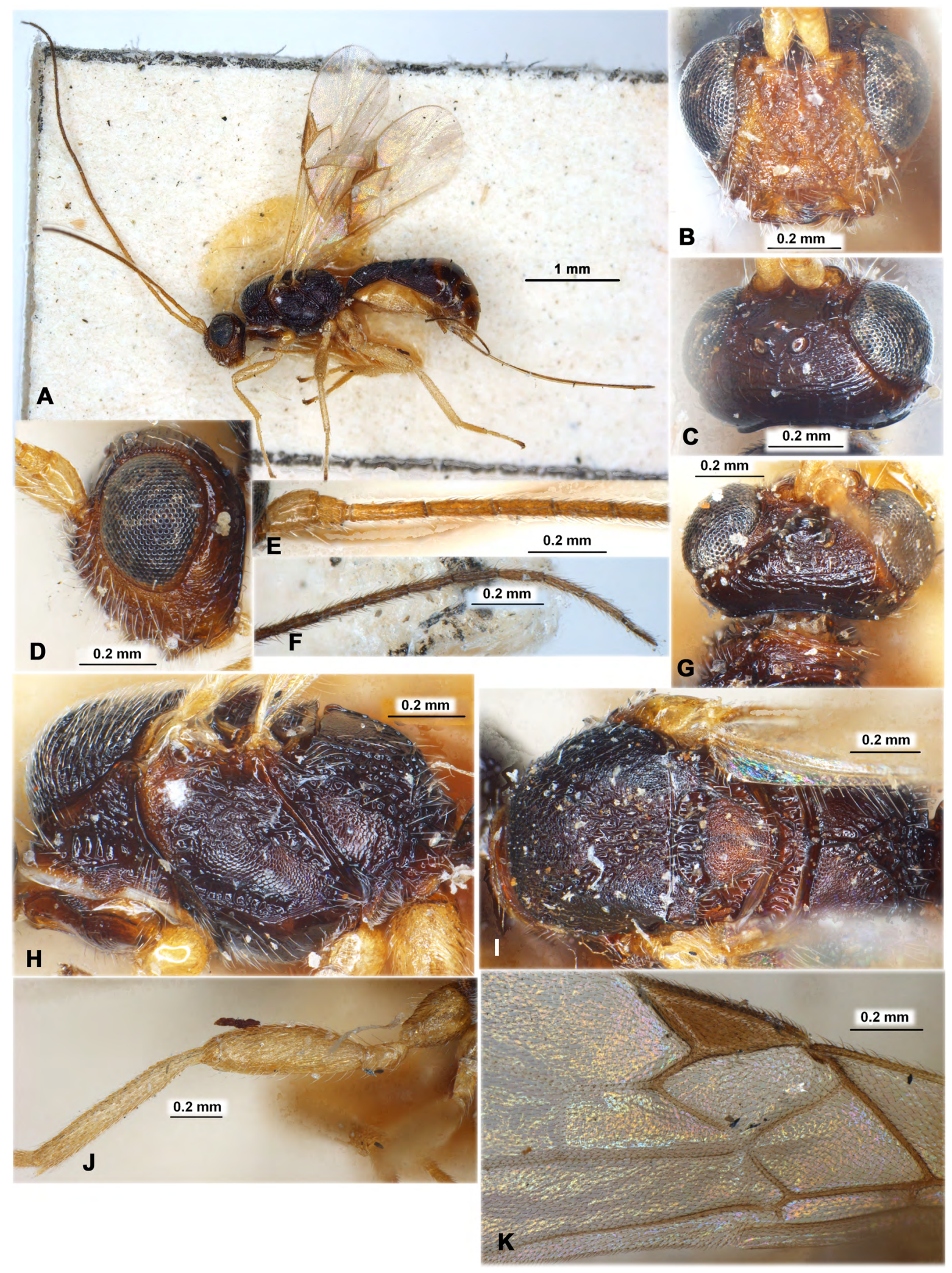

Fig. 52. Rhacontsira mozambiquensis sp. nov.,, , holotype, MNHN (A-F, H-K) and paratype, ZISP (G). A. Habitus, lateral view. B. Head, front view. C, G. Head, dorsal view. D. Head, lateral view. E. Basal segments of antenna. F. Apical segments of antenna. H. Mesosoma, lateral view. I. Mesosoma, dorsal view. J. Hind leg, lateral view. K. Median part of fore wing. 

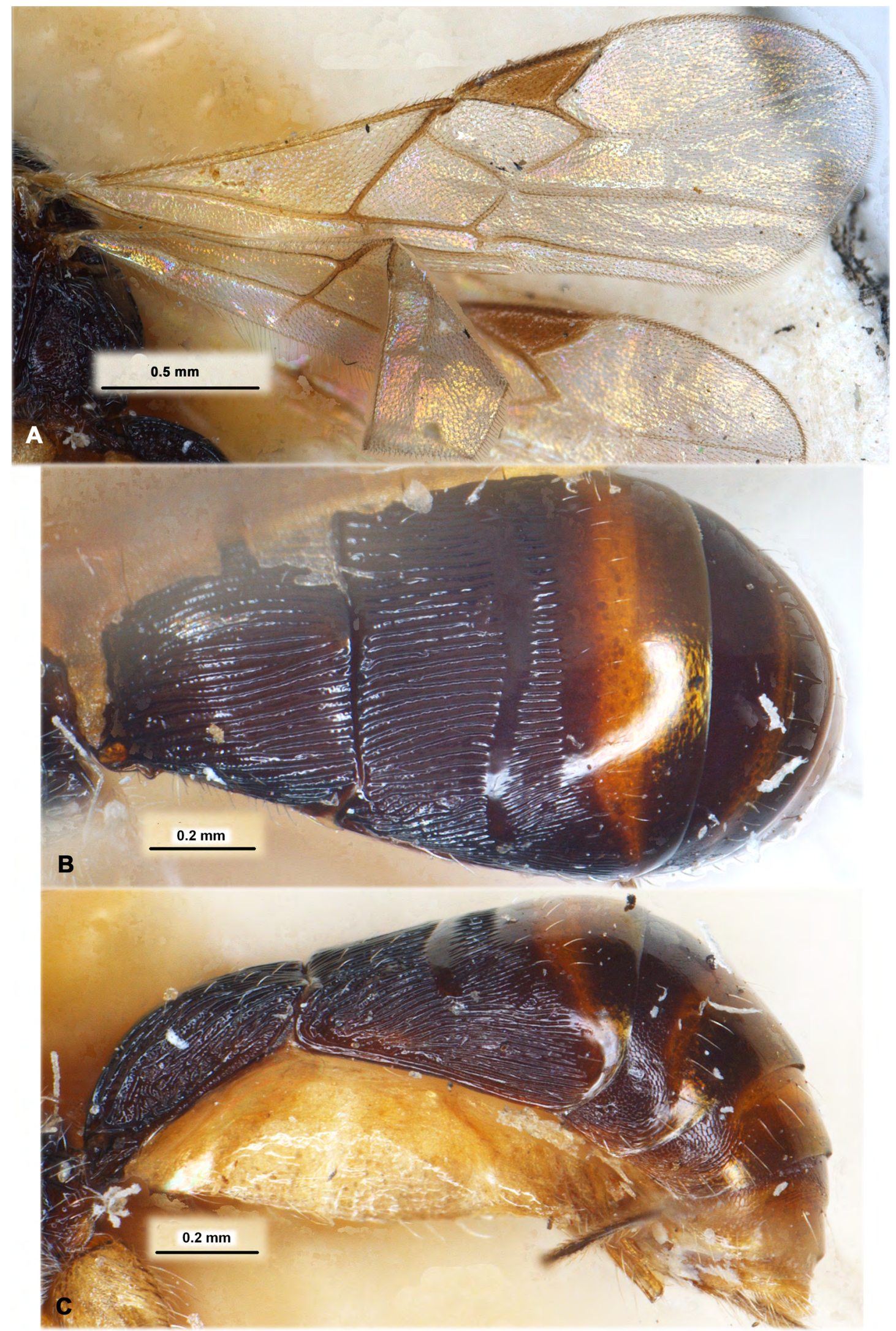

Fig. 53. Rhacontsira mozambiquensis sp. nov., + , holotype, MNHN. A. Wings. B. Metasoma, dorsal view. C. Metasoma, lateral view. 
distinct, shallow, crenulate with rugulosity, straight, oblique, connected with prepectal carina anteriorly, running along anterior 0.6 length of lower part of mesopleuron.

WING. Fore wing 2.8-3.0 times as long as its maximum width. Pterostigma 3.1-3.5 times as long as wide. Radial vein (r) arising almost from middle of pterostigma or weakly before it. Metacarpus (1-R1) 1.5 times as long as pterostigma. Second radial abscissa (3-SR) 1.8-2.0 times as long as first abscissa (r), 0.40-0.45 times as long as the straight third abscissa (SR1), 0.9 times as long as first radiomedial vein (2-SR). Second radiomedial (submarginal) cell not widened towards apex, 2.5-2.7 times as long as maximum width, 1.3-1.4 times as long as narrow brachial (subdiscal) cell. Recurrent vein (m-cu) weakly antefurcal. Parallel vein (CU1a) arising almost from middle of distal margin of brachial (subdiscal) cell. Distance from nervulus (cu-a) to basal (1-M) vein 0.75 times nervulus (cu-a) length. Hind wing 4.3-4.6 times as long as wide. First costal abscissa (C+SC+R) 0.7-0.8 times as long as second abscissa $(1-\mathrm{SC}+\mathrm{R})$. First abscissa of mediocubital vein $(\mathrm{M}+\mathrm{CU})$ 0.8-0.9 times as long as second abscissa (1-M). Recurrent vein $(\mathrm{m}-\mathrm{cu})$ weakly curved, strongly oblique, weakly antefurcal or postfurcal.

LEGS. Hind femur 3.0-3.2 times as long as wide. Hind tarsus almost as long as hind tibia. Hind basitarsus 0.7 times as long as second-fifth segments combined; second tarsal segment $0.4-0.5$ times as long as basitarsus, 1.1-1.2 times as long as fifth segment (without pretarsus).

Metasoma. Length 1.1-1.2 times as long as mesosoma and head combined. First tergite with distinct dorsope, with short subpointed and directed downwards basolateral processes, without spiracular tubercles, distinctly and linearly widened from base to subapex. Maximum width of first tergite 2.4-2.8 times minimum width; length $0.8-0.9$ times its apical width. Second tergite with very shallow and transverse sinuate furrow in posterior quarter separating short transverse apical area. Length of second tergite about 0.5 times its basal width, almost equal to length of third tergite. Second suture shallow, weakly curved and crenulate. Ovipositor sheath 1.2-1.6 times as long as metasoma, 1.8-2.0 times as long as mesosoma, $0.8-0.9$ times as long as fore wing.

SCULPTURE AND PUBESCENCE. Vertex distinctly curvedly transverse striate with fine rugulosity between striae; frons rugose-granulate, with curved longitudinal carinae laterally and posteriorly; face entirely coarsely rugose-reticulate with granulation. Mesoscutum entirely densely granulate and partly with fine rugulosity, partly granulae situated in curved transverse lines; rugose-reticulate and sometimes with undulate longitudinal striation in medio-posterior 0.3 of mesoscutum. Scutellum entirely densely granulate. Mesopleuron densely granulate, with rugosity in upper quarter. Propodeum with densely granulate with rugulosity (along carinae) basolateral areas delineated by distinct carinae; rest part of propodeum coarsely rugose-reticulate; areola indistinct. Hind coxa densely granulate, dorsally with distinct transverse curved striation. Hind femur finely and very densely granulate. First tergites entirely and second mostly (except smooth apical area) distinctly and densely longitudinally striate with fine reticulation between striae sometimes. Third tergite laterally almost entirely densely striate and partly with reticulation, its remainder smooth; fourth and fifth tergites laterally densely reticulate-coriaceous; these tergites medially widely smooth. Remaining tergites smooth. Vertex in sparse and short semi-erect setae, almost glabrous anteriorly. Mesoscutum entirely with short, dense and semi-erect white setae. Metapleural flange posteriorly with very dense pale pubescence. Hind tibia with short and dense semierect white setae; length of these setae about half maximum width of hind tibia.

CoLour. Body black to dark reddish brown; prothorax or only propleuron and posterior half of metasoma reddish brown. Head mostly dark reddish brown, face and malar space pale brown. Antenna yellow to brownish yellow in basal half, brown to dark brown in apical half. Palpi yellow to pale yellow. Legs entirely yellow. Fore wing hyaline. Pterostigma brown, paler apically. 
Male

Unknown.

\section{Diagnosis}

This Afrotropical species is very similar to the Eastern Palaearctic Rh. insulicola Belokobylskij \& Maeto, but differs from it by having the first flagellar segment 5.2 times as long as its apical width and 1.1 times as long as second segment (vs only 3.8-4.3 times as long as apical width and not longer than second segment), ocelli arranged in triangle with base 1.3 times its sides (vs in almost equilateral triangle), frons rugose-granulate and with curved longitudinal carinae laterally (vs densely obliquely striate and without lateral carinae), and the first metasomal tergite 0.8 times as long as its maximum width (vs almost as long maximum width).

\section{Distribution}

Mozambique.

Rhacontsira saigonensis sp. nov. urn:1sid:zoobank.org:act:3561576B-7C4D-428E-B210-B389648FD1F3

Figs $54-55$

\section{Etymology}

Named after the type locality of the species, Saigon (former name for Ho Chi Minh City).

\section{Material examined}

\section{Holotype}

VIETNAM • $q$; “Saigon [= Ho Chi Minh City], IV.[19]50, J. Barbier”, “2305”; MNHN.

\section{Paratypes}

VIETNAM • 2 우; same collection data as for holotype, but numbers 2304, 2309; MNHN, ZISP・ 1 ○; same collection data as for holotype, but number 2308; MNHN.

\section{Description}

\section{Female}

Measurements. Body length $1.7 \mathrm{~mm}$; fore wing length 1.4-1.5 mm.

HEAD. Width 1.5-1.7 times its median length, 1.3-1.4 times maximum width of mesoscutum. Head behind eyes (dorsal view) distinctly roundly narrowed. Transverse diameter of eye 2.3-2.4 times as long as temple (dorsal view). Ocelli medium-sized, arranged in triangle with base 1.2-1.3 times its sides. POL about 1.5 times OD, 0.4-0.5 times OOL. Eye glabrous, 1.20-1.25 times as high as broad. Malar space $0.30-0.35$ times height of eye and almost equal to basal width of mandible. Face width 0.85-0.90 times height of eye and 1.0-1.1 times height of face and clypeus combined. Malar suture absent. Clypeus rather high, with distinct lower flange. Clypeal suture distinct. Hypoclypeal depression round, its width 0.9-1.0 times distance from edge of depression to eye, 0.4 times width of face. Occipital carina coarse, complete dorsally, fused below with hypostomal carina above base of mandible. Hypostomal flange rather wide. Head in front view distinctly and weakly-roundly narrowed below eyes. Antennae slender, filiform, 18-20-segmented, 1.2-1.3 times as long as body. Scapus 1.4-1.6 times as long as its maximum width. First flagellar segment 5.0-6.0 times as long as its apical width, 0.9-1.0 times as long as second segment. Penultimate segment 4.0-4.5 times as long as width, 0.8-0.9 times as long as first flagellar and apical segments; the latter weakly acuminate apically. 
BELOKOBYLSKIJ S.A. \& ZALDÍVAR-RIVERÓN A., Reclassification of the Rhaconotini

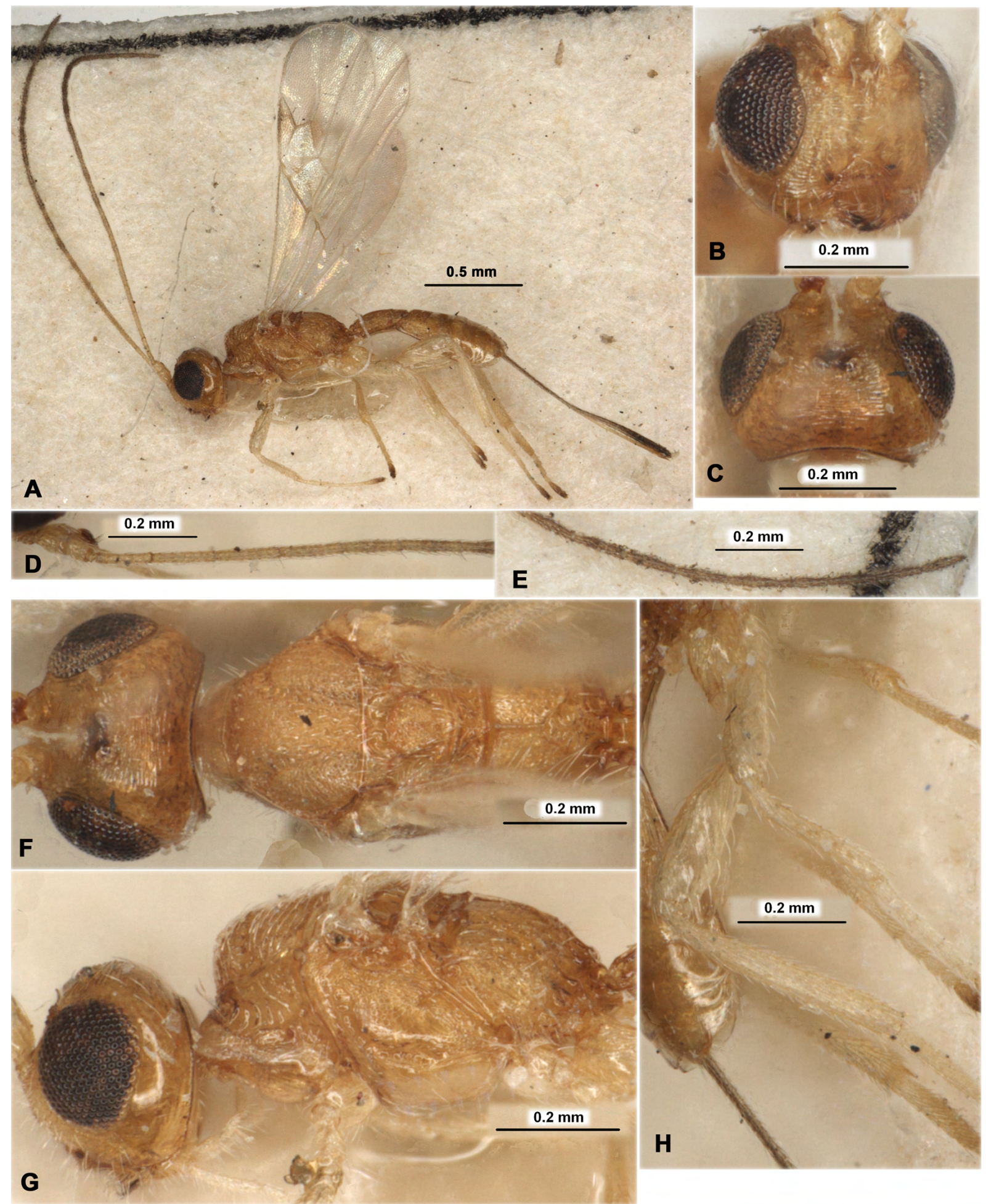

Fig. 54. Rhacontsira saigonensis sp. nov.,, , holotype, MNHN. A. Habitus, lateral view. B. Head, front view. C. Head, dorsal view. D. Basal segments of antenna. E. Apical segments of antenna. F. Head and mesosoma, dorsal view. G. Head and mesosoma, lateral view. H. Hind leg, lateral view. 


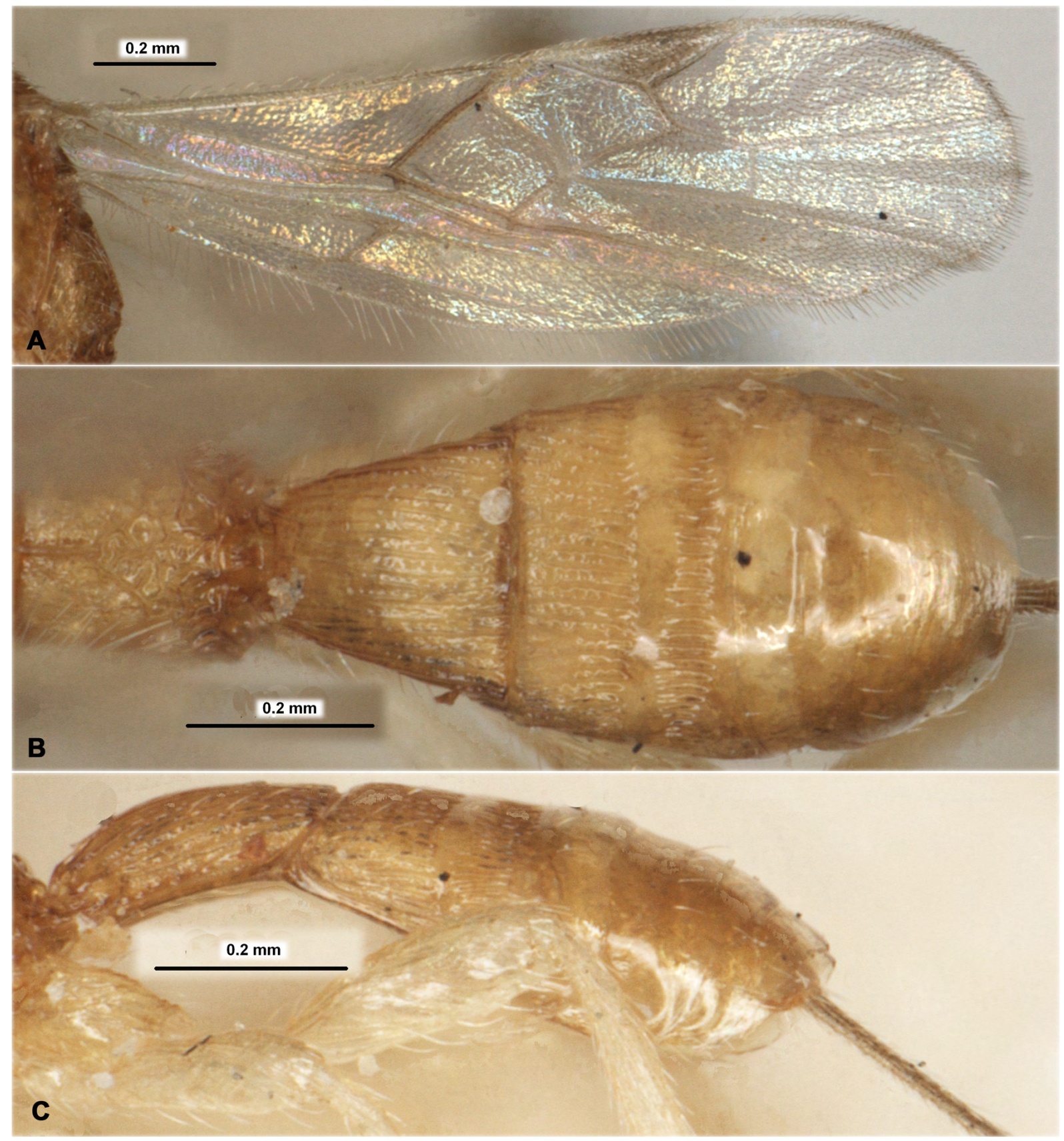

Fig. 55. Rhacontsira saigonensis sp. nov.,, , holotype, MNHN. A. Wings. B. Propodeum and metasoma, dorsal view. C. Metasoma, lateral view. 
Mesosoma. Mesosoma not depressed, 1.7-1.9 times as long as its high. Pronotum with distinct pronotal carina situated more closely to mesoscutum, but not fused with it. Mesoscutum highly and almost perpendicularly elevated above pronotum. Median lobe of mesoscutum not protruding forward. Notauli complete, rather deep anteriorly and shallow posteriorly, crenulate-rugulose. Prescutellar depression rather deep, short medially and longer laterally, with high median and two weak and curved lateral carinae, almost smooth between carinae, $0.3-0.4$ times as long as weakly convex scutellum. Metanotum with very short and subpointed dorsal tooth (lateral view). Subalar depression shallow, wide, rugosestriate. Sternaulus distinct, rather deep, crenulate and with fine rugulosity, weakly curved, oblique, connected anteriorly with prepectal carina, running along anterior 0.6 of lower part of mesopleuron.

WING. Fore wing 3.2-3.3 times as long as its maximum width. Pterostigma 3.6-4.2 times as long as wide. Radial vein (r) arising almost from middle of pterostigma. Metacarpus (1-R1) 1.3-1.5 times as long as pterostigma. Second radial abscissa (3-SR) 1.8-2.3 times as long as first abscissa (r), 0.35 times as long as the straight third abscissa (SR1), 0.9-1.0 times as long as first radiomedial vein (2-SR). Second radiomedial (submarginal) cell not widened towards apex, 2.8-3.0 times as long as maximum width, 1.4-1.5 times as long as narrow brachial (subdiscal) cell. Recurrent vein (m-cu) weakly antefurcal. Parallel vein (CU1a) arising distinctly before middle of distal margin of brachial (subdiscal) cell, sometimes almost interstitial. Distance from nervulus (cu-a) to basal (1-M) vein $0.5-0.7$ times nervulus (cu-a) length. Hind wing 6.2-6.5 times as long as wide. First costal abscissa $(\mathrm{C}+\mathrm{SC}+\mathrm{R}) 0.5-0.7$ times as long as second abscissa (1-SC+R). First abscissa of mediocubital vein $(\mathrm{M}+\mathrm{CU})$ about 0.7 times as long as second and third abscissae combined (1-M). Recurrent vein (m-cu) weakly curved, strongly oblique, distinctly antefurcal.

LeGs. Hind femur 3.2-3.5 times as long as wide. Hind tarsus $0.9-1.0$ times as long as hind tibia. Hind basitarsus 0.7 times as long as second-fifth segments combined; second tarsal segment $0.45-0.50$ times as long as basitarsus, 1.1 times as long as fifth segment (without pretarsus).

Metasoma. Length 0.9-1.0 times as long as mesosoma and head combined. First tergite with distinct dorsope, with very short rounded and directed downwards basolateral processes, without spiracular tubercles, distinctly and linearly widened from base to apex. Maximum width of first tergite 2.0-2.3 times its minimum width; length 1.0-1.1 times its apical width. Second tergite with rather distinct shallow and transverse furrow in posterior third separating relatively long transverse apical area. Length of second tergite 0.6 times its basal width, almost equal to length of third tergite. Second suture rather deep, weakly curved and crenulate. Ovipositor sheath 1.3-1.5 times as long as metasoma, 1.6-1.9 times as long as mesosoma, $0.6-0.8$ times as long as fore wing.

SCULPTURE AND PUBESCENCE. Vertex entirely distinctly curvedly transverse striate, sometimes with additional weak rugulosity between striae, finely striate to smooth posteriorly; frons laterally weakly transversely striate with rugulosity between striae, widely smooth medially; area around ocelli sometimes reticulate-granulate. Face entirely transversely striate, sometimes smooth in median vertical stripe; temple smooth. Mesoscutum densely granulate with reticulation, rugose with longitudinal undulate striation in its medio-posterior third. Scutellum entirely densely granulate. Mesopleuron densely rugose-reticulate, becoming finer towards its medial part or almost smooth on small or large median area. Propodeum with areas delineated by distinct carinae, basolateral areas densely granulate-reticulate with rugulosity along carinae or sometimes weakly reticulate-coriaceous; areola long and rather narrow or wide and reticulate; rest part of propodeum coarsely rugose-reticulate. Hind coxa densely curvedly transversely striae with reticulation. Hind femur mainly smooth, dorsally finely reticulate-coriaceous and sometimes with striation. First tergites entirely and second mostly (except smooth apical area) evenly and densely longitudinally striate with weak reticulation between striae. Third tergite laterally in basal half or two thirds densely striate, smooth on remaining part. Remaining tergites smooth. Vertex with very sparse 
short semi-erect white setae, almost glabrous anteriorly. Mesoscutum entirely with short, dense and semi-erect white setae. Metapleural flange posteriorly with sparse white pubescence. Hind tibia with short and dense semi-erect white setae; length of these setae about half maximum width of hind tibia.

CoLour. Body entirely brownish yellow. Antenna yellow in basal quarter or half, brownish yellow to brown on other part. Palpi pale yellow. Legs entirely yellow, apical segments of tarsi mainly dark brown. Fore wing hyaline. Pterostigma brown, pale in basal third and apically.

\section{Male}

MeASuREMENTs. Body length $1.7 \mathrm{~mm}$; fore wing length $1.3 \mathrm{~mm}$.

HEAD. Antennae weakly setiform, 19-segmented.

SCULPTURE AND PUBESCENCE. Vertex almost smooth in posterior third.

Metasoma. First tergite longer and narrow; its length 1.4 times apical width. Length of second tergite 0.75 times its basal width, 0.9 times length of third tergite. Fourth tergite shortly crenulate basally. Otherwise very similar to female.

\section{Diagnosis}

This new species is similar to the Vietnamese Rh. sculpturator, but distinctly differs from it by having the vertex evenly transverse striate and with fine reticulation (vs undulately striate with coarse reticulation), temple smooth (vs rugose-reticulate), parallel vein (CU1a) of fore wing arising distinctly before middle of distal margin of brachial (subdiscal) cell (vs almost from middle), hind femur mainly smooth, only dorsally finely reticulate-coriaceous and sometimes with striation (vs densely striate), fourth and fifth metasomal tergite laterally almost smooth (vs distinctly widely striate), body entirely brownish yellow (vs mainly black with reddish tint), and pterostigma brown, pale in basal third and apically (vs almost entirely dark brown).

\section{Distribution}

Vietnam.

Rhacontsira toamasina sp. nov. urn:1sid:zoobank.org:act:3C18B949-1829-4E3B-82AB-E3D148091ABB

Figs $56-57$

\section{Etymology}

Named after the type locality of the species, Toamasina Mts in Madagascar.

\section{Material examined}

Holotype

MADAGASCAR • + ; “Madagascar, Toamasina Montagne, D’Anjanaharibe, 7-6 km $341^{\circ} \mathrm{N} \mathrm{NW}$ Amananitelo. 8-12/III/2003, elv. 470 m. Calif. Acad. Sci. 15¹1'18" S 49³6'54" E, BLF 8005, Caslot 014065, Pan. Tr., rain forest. Coll. Fisher, Griswold et al., CNIN1235”; ZISP.

\section{Description}

Female

MeASurements. Body length $5.0 \mathrm{~mm}$; fore wing length $3.5 \mathrm{~mm}$. 


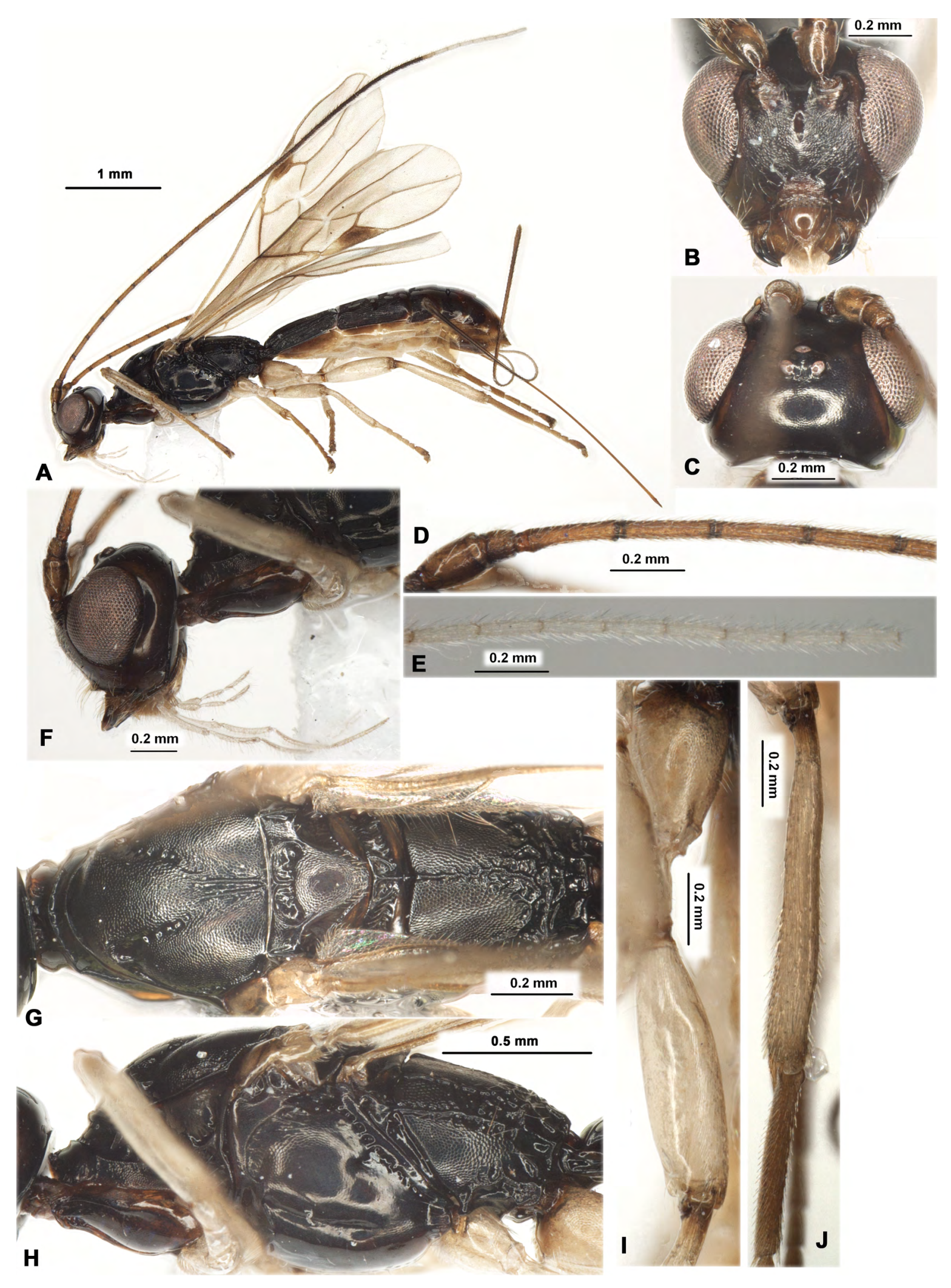

Fig. 56. Rhacontsira toamasina sp. nov., + , holotype, ZISP. A. Habitus, lateral view. B. Head, front view. C. Head, dorsal view. D. Basal segments of antenna. E. Apical segments of antenna. F. Head, lateral view. G. Mesosoma, dorsal view. H. Mesosoma, lateral view. I. Hind coxa and femur, lateral view. J. Hind tibia and basitarsus, lateral view. 


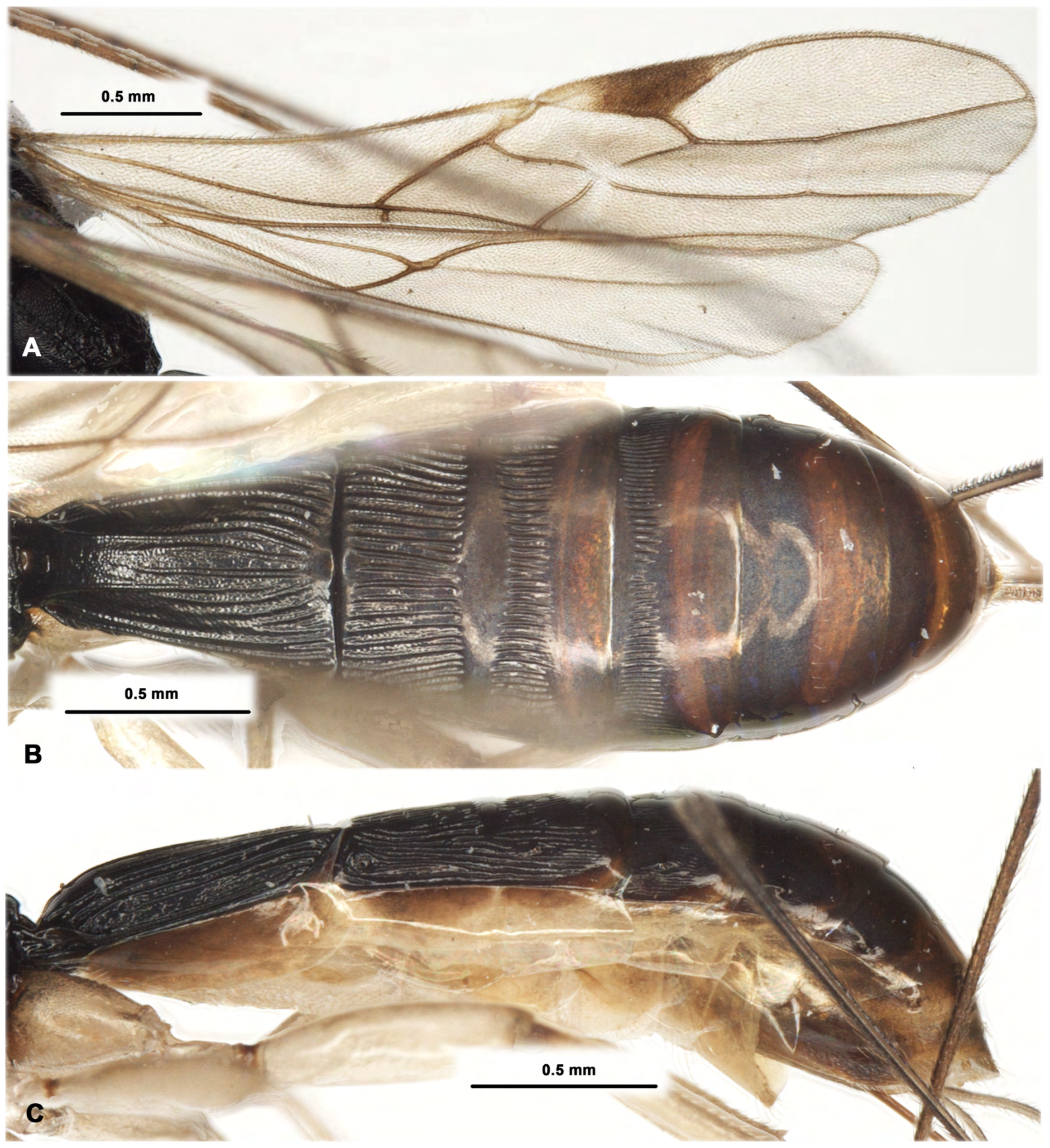

Fig. 57. Rhacontsira toamasina sp. nov., $q$, holotype, ZISP. A. Wings. B. Metasoma, dorsal view. C. Metasoma, lateral view. 
HeAD. Width 1.5 times its median length, 1.2 times maximum width of mesoscutum. Head behind eyes (dorsal view) distinctly roundly narrowed. Transverse diameter of eye 2.3 times as long as temple (dorsal view). Ocelli medium-sized, arranged in triangle with base 1.3 times its sides. POL almost equal to OD, 0.4 times OOL. Eye glabrous, 1.2 times as high as broad. Malar space 0.3 times height of eye and 0.8 times basal width of mandible. Face width 0.8 times height of eye and almost equal to height of face and clypeus combined. Clypeus rather high. Clypeal suture distinct and complete. Hypoclypeal depression round, its width 0.8 times distance from edge of depression to eye, 0.4 times width of face. Occipital carina coarse, complete dorsally, fused below with hypostomal carina above base of mandible. Hypostomal flange relatively wide. Head in front view distinctly and weakly-roundly narrowed below eyes. Antennae slender, filiform, more than 36-segmented (apical segments missing). Scapus 1.6 times as long as maximum width. First flagellar segment 4.7 times as long as its apical width, 1.1 times as long as second segment. Subapical segments 3.5 times as long as their width.

Mesasoma. Mesosoma not depressed, weakly elongate, 2.1 times as long as its high. Pronotum with distinct pronotal carina situated submedially. Mesoscutum distinctly and gently-roundly elevated above pronotum. Median lobe of mesoscutum weakly protruding forward, with short but distinct anterolateral corners, without longitudinal furrow. Notauli complete, distinct anteriorly, shallower in posterior quarter, sparsely crenulate. Prescutellar depression rather shallow, short, with distinct three carinae, finely rugulose to smooth between carinae, about 0.3 times as long as very weakly convex scutellum. Metanotum with short, thickened and apically rounded tooth (lateral view). Subalar depression rather shallow, partly crenulate and densely coriaceous. Sternaulus distinct, shallow, narrow, smooth, almost straight, weakly oblique, connected with prepectal carina anteriorly, running along anterior 0.7 length of lower part of mesopleuron.

WING. Fore wing 4.2 times as long as its maximum width. Pterostigma 4.0 times as long as wide. Radial vein (r) arising weakly behind middle of pterostigma. Metacarpus (1-R1) 1.3 times as long as pterostigma. Second radial abscissa (3-SR) 3.0 times as long as first abscissa (r), 0.6 times as long as the straight third abscissa (SR1), 1.3 times as long as first radiomedial vein (2-SR). Second radiomedial (submarginal) cell not widened towards apex, 3.5 times as long as maximum width, 1.3 times as long as narrow brachial (subdiscal) cell. Recurrent vein (m-cu) weakly antefurcal. Parallel vein (CU1a) arising from upper 0.25 of distal margin of brachial (subdiscal) cell. Distance from nervulus (cu-a) to basal (1-M) vein 0.4 times nervulus (cu-a) length. Hind wing 5.3 times as long as wide. First costal abscissa $(\mathrm{C}+\mathrm{SC}+\mathrm{R}) 0.7$ times as long as second abscissa $(1-\mathrm{SC}+\mathrm{R})$. First abscissa of mediocubital vein $(\mathrm{M}+\mathrm{CU})$ 0.7 times as long as second abscissa $(1-\mathrm{M})$. Recurrent vein $(\mathrm{m}-\mathrm{cu})$ weakly curved, strongly oblique, interstitial.

LEGs. Hind femur 3.6 times as long as wide. Hind tarsus 1.15 times as long as hind tibia. Hind basitarsus 0.8 times as long as second-fifth segments combined; second tarsal segment 0.4 times as long as basitarsus, almost as long as fifth segment (without pretarsus).

Metasoma. Length 1.3 times as long as mesosoma and head combined. First tergite with distinct dorsope, with short rounded and directed downwards basolateral processes, with very small spiracular tubercles, distinctly and linearly widened from base to subapex. Maximum width of first tergite 2.6 times minimum width; length 1.4 times its apical width, 1.5 times length of propodeum. Second tergite with shallow and weakly curved transverse furrow in posterior third separating rather short transverse lenticular apical area. Length of second tergite 0.7 times its basal width, almost twice length of third tergite. Second suture shallow, weakly curved and widely crenulate. Ovipositor sheath 0.9 times as long as metasoma, 1.4 times as long as mesosoma, 0.7 times as long as fore wing. 
SCULPTURE AND PUBESCENCE. Vertex mainly finely coriaceous, partly smooth; frons smooth; face mainly densely granulate, below becoming coriaceous to smooth; frons smooth. Mesoscutum entirely densely and rather finely granulate, without rugulosity along notauli, with two subparallel striae in its medio-posterior quarter. Scutellum entirely densely granulate. Mesopleuron mostly smooth, coriaceous to finely granulate dorsally. Propodeum with long granulate basolateral areas delineated by distinct carinae; posterior part of propodeum coarsely rugose-reticulate; areola indistinct. Hind coxa densely and finely granulate in dorsal half, coriaceous to almost smooth in lateral and ventral parts. Hind femur finely and obliquely aciculate dorsally, mostly smooth. First tergite mostly curvedly striate and densely coriaceous between striae, almost smooth medio-posteriorly. Second tergite mostly distinctly striate, smooth or almost smooth narrow basally and in apical area. Third and fourth tergites medially in basal $0.2-0.3$ and laterally in basal $0.7-0.5$ distinctly striate, smooth on remaining parts. Remainder tergites smooth. Vertex in entirely glabrous. Mesoscutum widely along notauli and very sparsely laterally with very short pale setae, glabrous on wide median areas on all lobes. Mesopleuron almost entirely glabrous. Metapleural flange without pubescence. Hind tibia with very short and dense semi-erect yellowish setae; length of these setae $0.2-0.3$ times maximum width of hind tibia.

CoLOuR. Body mostly black and partly with reddish tint or areas; metasoma dark reddish brown in posterior half dorsally, ventrally widely brownish yellow to yellow. Antenna reddish brown in basal third, almost black submedially, with more than nine white apical segments. Palpi pale yellow. Legs mostly yellow, all tarsi brownish with dark apical segments. Fore wing faintly and evenly infuscate. Pterostigma dark brown, yellow to pale brown in basal third.

\section{Male \\ Unknown. \\ Diagnosis}

This new species morphologically resembles Rh. heterospiloides Belokobylskij, 1988 but distinctly differs from it by the elongate body (vs robust), vertex finely coriaceous (vs mainly transversely striate), apical segments of antenna white (vs black), second metasomal tergite with very short and smooth basal area (vs without area).

\section{Distribution}

Madagascar.

Genus Troporhaconotus gen. nov. urn:1sid:zoobank.org:act:6D821B9C-95EC-4CAC-873F-1FA1F40902A6

Rhaconotus jacobsoni species group - Belokobylskij 2001: 134. — Qanh \& Long 2019: 37.

\section{Etymology}

From "tropics", the climatic region where this taxon appears to be restricted, and the generic name "Rhaconotus". Gender: masculine.

\section{Type species}

Pseudospathius jacobsoni Szépligeti, 1908.

\section{Description (Figs 58-59)}

HEAD. Head subglobular. Vertex densely and finely granulate or granulate-reticulate. Ocelli arranged often in almost equilateral triangle. Frons almost flat and without median keel. Eyes with distinct 
emargination opposite eyes, glabrous. Occipital carina complete dorsally, not joining ventrally with hypostomal carina being obliterated at short distance. Malar suture absent. Clypeal suture distinct and complete. Clypeus with more or less distinct ventral flange. Hypoclypeal depression medium size, round. Postgenal bridge rather wide. Palps rather short; maxillary palps 6-segmented, labial palps 4-segmented. Scapus wide and rather short, without apical lobe or basal constriction. First flagellar segment subcylindrical, almost straight, always longer than second segment.

Mesosoma. Mesosoma long and narrow, 2.5-3.0 times as long as its high. Pronotum dorsally distinctly convex, with more or less distinct pronotal carina in anterior 0.3-0.4. Mesonotum not highly and weakly gently-roundly elevated above pronotum (lateral view); its all three lobes medially widely glabrous. Notauli complete, deep, crenulate. Prescutellar depression relatively long, with median carina, rugulose. Scuto-scutellar suture rather distinct. Scutellum with distinct lateral carinae at least in anterior half. Metanotum often with long and acuminate median tooth (lateral view). Sternaulus (precoxal furrow) distinct, relatively deep, long, running along all length of lower part of mesopleuron, distinctly crenulate. Prepectal carina complete, enlarged below opposite fore coxa. Postpectal carina absent. Propodeum at least with long median and two lateral carinae, often basolateral areas not delineated posteriorly, areola indistinct; lateral tubercles and propodeal bridge absent.

Wings. Pterostigma bicolorated, mostly dark brown with pale its basal part. Radial vein (r) arising usually from or more or less behind middle of pterostigma. Radial (marginal) cell not shortened. Both radiomedial veins (2-SR, r-m) present. Recurrent vein (m-cu) distinctly postfurcal. Discoidal (discal) cell petiolate, narrow and long. Nervulus (cu-a) weakly postfurcal or sometimes subinterstitial. Mediocubital vein (M+CU1) rather distinctly curved distally to anal vein (2-1A). Parallel vein (CU1a) always interstitial. Brachial (subdiscal) cell closed usually before or (rarely) on level of recurrent vein (m-cu). Hind wing with three hamuli. Radial (marginal) cell without additional transverse vein (r). Medial (basal) cell rather narrow, but distinctly widened apically. Nervellus (cu-a) present. Submedial (subbasal) cell small. First abscissa of mediocubital vein $(\mathrm{M}+\mathrm{CU})$ distinctly shorter than second abscissa (1-M). Recurrent vein (m-cu) present, but often not pigmented.

LEGS. Fore tibiae with several distinct spines arranged in almost single line. Middle leg short and with short tarsal segments. Hind coxa with distinct basoventral corner and distinct, but small tubercle. Hind femur with rather distinct dorsal protuberances; femur of male distinctly broaded. Hind tibia with long, more or less dense and semi-erect setae. Hind basitarsus 0.6-0.7 times as long as second-fifth segments combined. Legs usually with dark and pale colour pattern.

Metasoma. Six dorsally visible and hardly sclerotised tergites. First tergite not petiolate, but very long, its dorsope rather large, basolateral lobes absent. Acrosternite of first segment 0.15-0.2 times as long as first tergite, its apical margin situated distinctly before level of spiracles. First and second tergites not fused immovably. Second tergite without basal area delineated posteriorly by furrow; apical area of second tergite present, long, coarsely sculptured, delineated anteriorly by relatively shallow and wide crenulate furrow and posteriorly by rather deep and wide curved second suture. Second-sixth tergites with separated laterotergites. Sixth tergite of female always enlarged, distinctly longer than fifth tergite, covered following apical segments, with usually distinctly convex its posterior margin, but only sometimes with more or less distinct incurvation posteriorly.

\section{Diagnosis}

This new genus differs from the remaining Rhaconotini genera by having more than five dorsally visible metasomal tergite and with distinctly enlarged sixth tergite covering following tergites, first metasomal tergite long, more than 2.0 times as long as its apical width, second tergite without basal area and always 
with apical lenticular area rather distinctly delineated anteriorly by deep and rather wide furrow, and mesosoma long with mesoscutum weakly and gently elevated above pronotum.

\section{Composition}

Troporhaconotus anivoranensis (Granger, 1949) comb. nov. (AF); T. asulcus (Shi \& Chen, 2004) comb. nov. (OR); T. ceylonicus (Belokobylskij, 2001) comb. nov. (OR); T. chinensis (Belokobylskij \& Chen, 2004) comb. nov. (OR); T. directus (Long, 2019) comb. nov. (OR); T. glaphyrus (Chen \& Shi, 2004) comb. nov. (OR); T. jacobsoni (Szépligeti, 1908) [= Rhaconotus filicornis (Enderlein, 1912)] comb. nov. (OR); T. laevigatus (Long, 2019) comb. nov. (OR); T. longithorax (Belokobylskij, 2001) comb. nov. (OR); T. robustus (Long, 2019) comb. nov. (OR); T. simulatus (Long, 2019) comb. nov. (OR); T. thayi Belokobylskij, 2001 comb. nov. (OR).

\section{Hosts}

Unknown.

\section{Distribution}

Afrotropical and Oriental regions.

\section{Remarks}

In Jasso-Martínez et al. (2019), the only included species of this genus, T. thayi (Belokobylskij, 2001), was nested at the base of a large clade with taxa having mainly five-tergited metasoma. Despite its absence of sharp morphological diagnostic characters, the above combination of features together with its phylogenetic affinities allowed us to propose the erection of this new genus.

Troporhaconotus jacobsoni (Szépligeti, 1908) gen. et comb. nov.

Figs $58-59$

Pseudospathius jacobsoni Szépligeti, 1908: 222.

Spathiohormius filicornis Enderlein, 1912: 22.

Pseudospathius jacobsoni - Nixon 1943: 437 (as probably Rhaconotus). - Shenefelt \& Marsh 1976: 1386.

Rhaconotus jacobsoni - Belokobylskij 2001: 133. - Belokobylskij et al. 2004: 89. - Yu et al. 2016. Platyspathius filicornis - Shenefelt \& Marsh 1976: 1385. — Yu et al. 2016.

Rhaconotus filicornis - Belokobylskij 1996a: 158; 1998b: 69; 2001: 135 (as synonym).

\section{Material examined}

\section{Holotype}

INDONESIA • + , holotype of Pseudospathius jacobsoni Szépligeti, 1908; "E. Jacobson, Semarang, Java, 1905", "Holotypus +, Pseudospathius jaconsoni Szepl. 1908”, "Hym. Typ. No 1620. Mus. Budapest", "Pseudospath Jacobsoni" [handwriting by Szépligeti]; HNHM.

\section{Other material}

SRI LANKA • 1 q, holotype of Spathiohormius filicornis Enderlein, 1912; “Ceylon, Paradna, W. Horn”, "Holotypus", "Spathiohormius filicornis Enderl. क, type, Dr. Enderlein det. 1912", "Type", "Rhaconotus filicornis (End.), det. Belokobylskij, 1992”, "Rhaconotus jacobsoni (Sz.), det. Belokobylskij, 2001”; SDEI. 


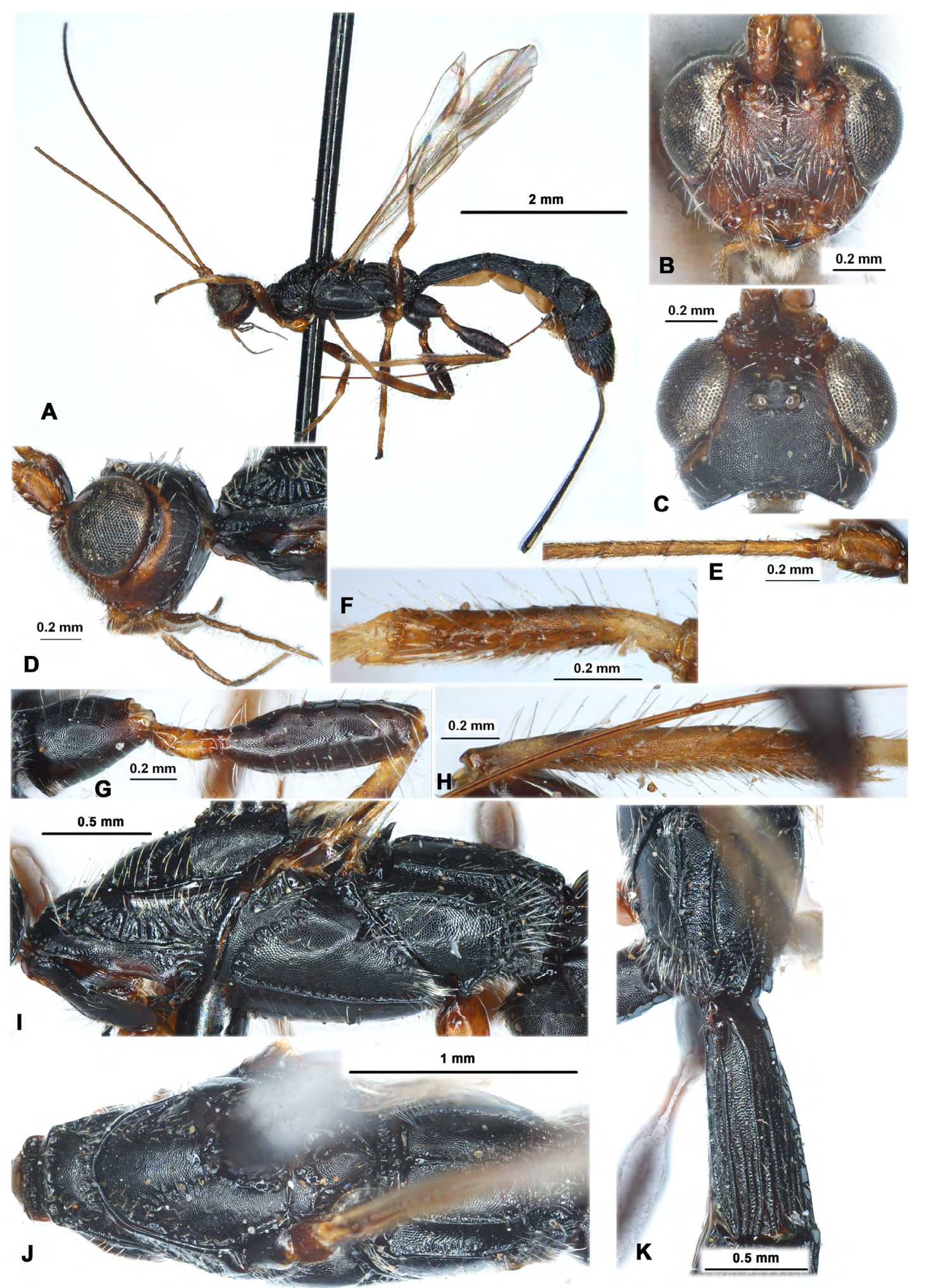

Fig. 58. Troporhaconotus jacobsoni (Szépligeti, 1908) gen. et comb. nov., + , holotype, HNHM. A. Habitus, lateral view. B. Head, front view. C. Head, dorsal view. D. Head, lateral view. E. Basal segments of antenna. F. Fore tibia. G. Hind coxa and femur, lateral view. H. Hind tibia, lateral view. I. Mesosoma, lateral view. J. Mesosoma, dorsal view. K. Propodeum and first metasomal tergite, dorsal view. 

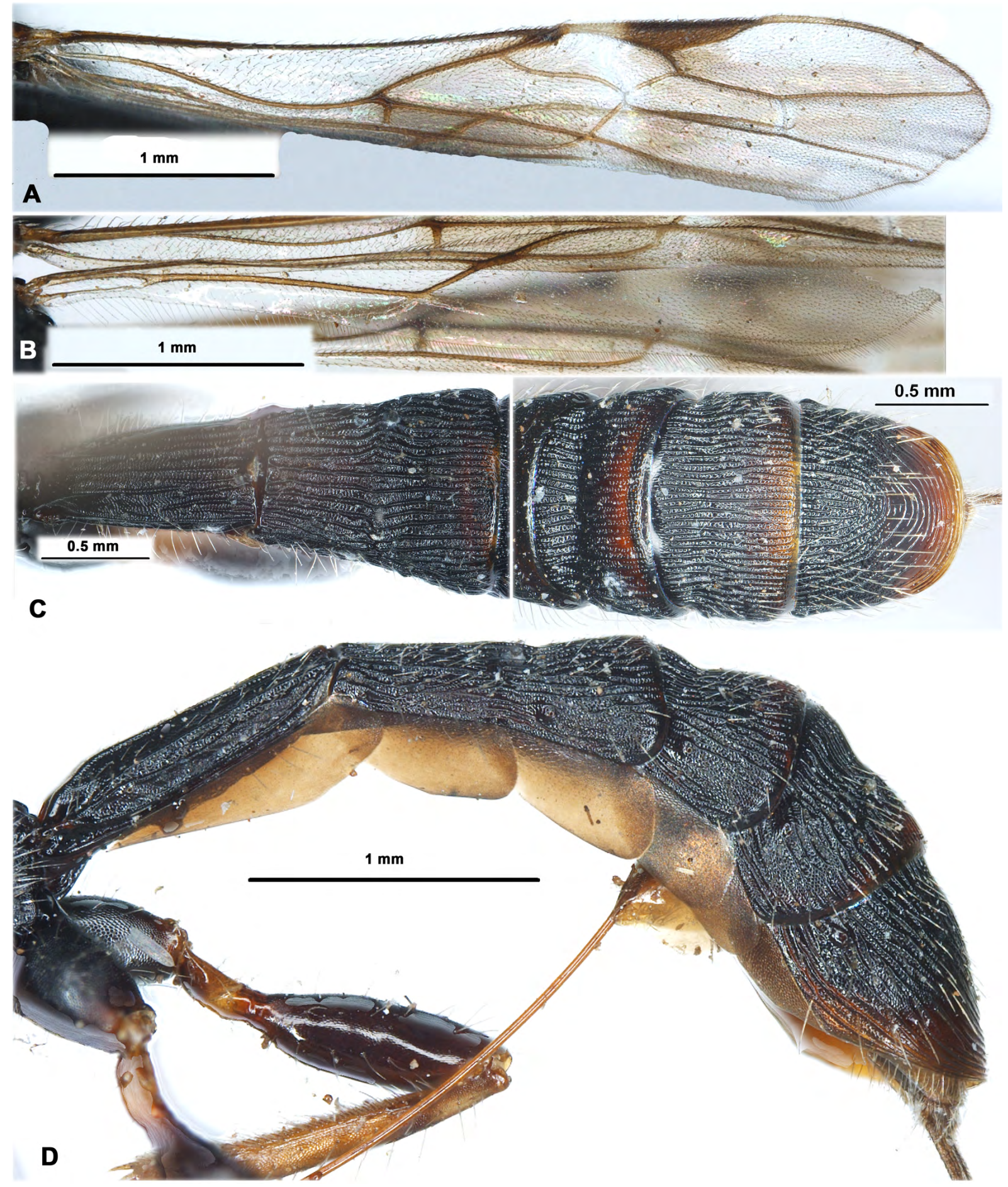

Fig. 59. Troporhaconotus jacobsoni (Szépligeti, 1908) gen. et comb. nov., + , holotype, HNMN. A. Fore wing. B. Hind wing. C. Metasoma, dorsal view. D. Metasoma, lateral view. 


\section{Description}

\section{Female}

MeASUREMENTS. Body length 7.0-7.5 mm; fore wing length 4.2-4.4 mm.

HEAD. Width 1.4-1.5 times its median length, 1.2-1.3 times width of mesoscutum. Head behind eyes (dorsal view) roundly narrowed. Transverse diameter of eye about 2.0 times as long as temple. Ocelli medium-sized, arranged in triangle with base 1.1-1.2 times its side; POL 0.7-1.0 times OD, 0.5-0.7 times OOL. Eye distinctly emarginated opposite antennal sockets, 1.15 times as high as broad. Malar space $0.30-0.35$ times height of eye, about 0.8 times basal width of mandible. Face width 0.9 times eye height and 1.2-1.3 times height of face and clypeus combined. Upper margin of clypeus situated distinctly higher than lower level of eyes. Width of hypoclypeal depression 0.7-0.9 times distance from depression to eye, 0.35-0.40 times width of face. Vertex weakly convex. Head below eyes (front view) distinctly and almost linearly narrowed. Antennae slender, weakly setiform, more then 35-segmented (apical segments missing). Scapus 1.6-1.7 times as long as its maximum width, about 4.0 times length of pedicel. First flagellar segment 4.8-5.0 times as long as its apical width, almost as long as second segment. Submedian segments 3.3-3.5 times as long as wide.

Mesosoma. Length 2.4-2.6 times its height. Pronotal carina high and situated on or weakly behind middle of pronotum (dorsal view). Mesoscutum (dorsal view) 1.1 times as long as wide. Notauli rather deep in anterior 0.3 , shallow on remaining part, rather wide, densely and distinctly crenulate with additional small and very dense granulation almost entirely. Median lobe of mesoscutum distinctly convex anteriorly. Prescutellar depression with three distinct carinae, finely rugulose to smooth on remaining parts, distinctly oblique from middle to both sides along its posterior margin, medially $0.2-0.3$ times as long as scutellum. Scutellum distinctly convex, its maximum width 1.2 times median length. Subalar depression rather densely and distinctly crenulate, below fused with anterior margin of sternaulus. Sternaulus (precoxal furrow) entirely distinctly and more or less densely crenulate. Propodeum weakly and almost linearly sloping backward (lateral view). Metapleural flange posteriorly with rather dense white setae.

WINGS. Length of fore wing 4.7-4.8 times its maximum width. Pterostigma 5.5 times as long as wide. Metacarpus (1-R1) 1.1 times as long as pterostigma. Radial (marginal) cell 3.7 times as long as wide. Radial vein (r) arising almost from middle of pterostigma. First radial abscissa (r) forming very obtuse angle with second abscissa (3-SR). Second radial abscissa (3-SR) 3.1-3.3 times as long as first abscissa (r), about 0.5 times as long as the straight third abscissa (SR1), 1.5-1.7 times as long as second radiomedial vein $(\mathrm{r}-\mathrm{m})$. Second radiomedial (submarginal) cell not widened distally, 3.4-3.5 times as long as wide, 0.8-0.9 times as long as the rather narrow brachial (subdiscal) cell. First medial abscissa $(1-\mathrm{SR}+\mathrm{M})$ distinctly sinuate. Recurrent vein $(\mathrm{m}-\mathrm{cu})$ 4.8-5.0 times as long as second abscissa of medial vein $(2-\mathrm{SR}+\mathrm{M}), 0.6-0.7$ times as long as first radiomedial vein (2-SR). Mediocubital vein $(\mathrm{M}+\mathrm{CU} 1)$ strongly curved in apical half. Medial (basal) cell sparsely setose. Distance from nervulus (cu-a) to basal (1-M) vein 0.2-0.3 times nervulus (cu-a) length. Brachial (subdiscal) cell closed weakly before recurrent vein $(\mathrm{m}-\mathrm{cu})$; posterior bulla present but short; posterior abscissa of anal vein $(2-1 \mathrm{~A})$ (behind brachial vein (CU1b)) absent. Hind wing 6.5-6.7 times as long as wide. Recurrent vein (m-cu) weakly curved, strongly oblique towards base of wing.

LEGS. Hind coxa 1.5-1.6 times as long as wide (with tubercle), 0.75 times as long as propodeum, with very dense white setae around tubercle. Hind femur about 3.0 times as long as wide. Hind tarsus 0.9 times as long as hind tibia. Hind basitarsus with lower keel and with dense long setosity along keel. Second segment of hind tarsus $0.40-0.45$ times as long as basitarsus, about as long as fifth segment (without pretarsus). 
Metasoma. Length 1.2-1.3 times as long as head and mesosoma combined, with sixth visible tergites, remaining segments not protruding behind sixth tergite. First tergite long, distinctly, almost linearly and evenly widened from base to apex; its maximum width 1.6-1.8 times minimum basal width; length 2.4-2.6 times its apical width, 1.3-1.4 times length of propodeum. Apical area of second tergite $0.5-0.6$ times as long as remaining part of tergite. Median length of second tergite (with area) 1.2 times its basal width, about 2.0 times median length of third tergite. Sixth tergite strongly enlarged, weakly curved on posterior margin, without median emargination posteriorly and postero-ventral lobes. Sixth tergite 1.7-1.8 times as long as fifth tergite (till subbasal transverse furrow) and 1.8-2.0 times as long as fourth tergites. Ovipositor sheath $0.6-0.8$ times as long as metasoma, 1.0-1.3 times as long as mesosoma, $0.5-0.7$ times as long as fore wing.

SCULPTURE AND PUBESCENCE. Vertex densely granulate; frons densely granulate in posterior third, densely rugose in anterior two thirds; face densely granulate, transverse striate additionally on narrow median area; temple granulate-coriaceous in upper half and finely reticulate to almost smooth in lower half. Mesoscutum and scutellum very densely granulate; mesopleuron and metapleuron densely and small areolate-coriaceous, metapleuron coarsely rugose in posterior third. Propodeum densely granulatereticulate in anterior 0.5 (medially) or 0.7 (laterally), distinctly rugose, with distinct median (in basal half) and two lateral (almost complete) strong carinae. Hind coxa and femur densely granulate-reticulate. First tergite with distinctly separate and almost parallel dorsal carinae, with coarse and complete lateral carinae; coarsely striate with very dense and fine transverse striation or reticulation between striae, only granulate-reticulate basally on short area and laterally in basal half. Second-fifth tergites entirely distinctly striate with very dense and fine transverse striation or reticulation between striae. Sixth tergites in semi-round coarse striation, with additional small and dense reticulation in its basal half. Second-sixth tergites laterally distinctly striate with additional very dense reticulation. Vertex medially widely glabrous, with rather dense, long and semi-erect white setae arranged along posterior margin and laterally. Mesonotum glabrous on wide median areas on all lobes, with sparse, short semi-erect white setae arranged widely along notauli and in single line marginally. Mesopleuron mostly glabrous, with narrow line of long, rather dense and white setae along posterior margin. Hind tibia in inner ventral half with dense yellow setae, dorsally with rather sparse numerous long pale setae, these setae 0.7-1.0 times as long as maximum width of tibia. Laterotergites mainly glabrous.

CoLOUR. Body black, head around eyes rather narrowly posteriorly and widely below and anteriorly red, sometimes third-sixth metasomal tergites apically reddish. Antenna light reddish brown or reddish brown basally, evenly darkened toward apex. Palps reddish brown or dark brown, becoming pale apically. Fore and middle legs reddish brown or light reddish brown, coxae paler, trochanters and trochantelli, base of tibiae and tarsi (except dark fifth segments) yellow. In hind leg, coxa and femur black, trochanters, trochantellus and most part of tibia yellowish brown or light reddish brown, tibia yellow basally and distinctly infuscate subasally and apically; tarsus yellow, fifth segment mainly almost black. Ovipositor sheath black, brown basally. Fore wing distinctly and widely maculate, hyaline in basal third, in rather narrow transverse stripes below base of pterostigma, in anterior half of radial (marginal) cell and in apical margin of wing. Pterostigma dark brown, yellow in basal third.

Male

Unknown.

\section{Distribution}

Indonesia, Sri Lanka. 


\section{Discussion}

Here we have proposed a generic reclassification for the doryctine tribe Rhaconotini based both on phylogenetic relationships obtained in a previous molecular phylogenetic study (Jasso-Martínez et al. 2018), as well as on the subsequent examination of external morphological features, especially those that have been traditionally employed to define genera in the group. This updated phylogenetic-based classification for the tribe is thus supported by an estimate of phylogeny that can be tested in further studies including additional taxa, morphological features and/or genetic markers.

Our comprehensive morphological revision of all currently known rhaconotine taxa led us to recognise the following 15 genera within the tribe: Antespathius Belokobylskij, 1995, Arhaconotus Belokobylskij, 2000, Bathycentor Saussure, 1892, Euryphrymnus Cameron, 1910, Grangerdoryctes Belokobylskij, 2005, Ipodoryctes Granger, 1949 (with two subgenera, Afroipodoryctes subgen. nov. and Ipodoryctes s.str.), Leptorhaconotus Granger, 1949, Mimipodoryctes Belokobylskij, 2000, Neorhaconotus Belokobylskij, Iqbal \& Austin, 2004, Platyspathius, Viereck, 1911 (with two subgenera, Lenticularia van Achterberg, 2003 and Platyspathius s. str.), Pseudorhaconotus van Achterberg \& Shaw, 2010, Rhaconotinus Hedqvist, 1965 (with two subgenera, Hexarhaconotinus subgen. nov. and Rhaconotinus s. str.), Rhaconotus Ruthe, 1854 (with two subgenera, Pararhacon Belokobylskij, 2005 and Rhaconotus s. str.), Rhacontsira Belokobylskij ,1998 and Troporhaconotus gen. nov.

The main distinctive feature that characterises the Rhaconotini is the presence of a hardly sclerotized metasoma, which has separate laterotergites in the first five or six tergites that are usually entirely or occasionally only basally sculptured, with the fifth or sixth tergites usually covering the remaining ones. This perhaps represents the most distinctive morphological synapomorphy for the tribe. Members of the Rhaconotus s. str., Euryphrymnus, Neorhaconotus and Pseudorhaconotus, on the other hand, are the genera within the Rhaconotini with only five dorsally visible and usually widely sculptured metasomal tergites, with the fifth one distinctly enlarged. Our previous molecular phylogenetic study (Jasso-Martínez et al. 2018) helped us to confirm that the species with this unique, highly modified feature form a monophyletic group, which led us here to restrict the generic name Rhaconotus to most of them.

Arhaconotus and Mimipodoryctes have the first and second metasomal tergites fused and immovable (Belokobylskij 2001). This feature can be also observed in all members of the subfamilies Adeliinae and Cheloninae, as well as in a few braconid genera belonging to the subfamilies Doryctinae, Rogadinae, Lysiterminae, Braconinae, Gnamptodontinae and Brachistinae (Quicke \& van Achterberg 1990; van Achterberg 1993; Belokobylskij 1996b; Belokobylskij et al. 2015). Antespathius, on the other hand, was originally placed within the Spathiini (Belokobylskij, 1995) based on the main feature that was used to distinguish members of this tribe, a considerable petiolate first metasomal tergite with strongly elongated acrosternite (equal to $0.6-0.7$ times length of tergite). This feature, however, has been shown to have independently evolved in various doryctine genera (Zaldívar-Riverón et al. 2007, 2008). Within the Rhaconotini, an elongated acrosternite also occurs in species of Leptorhaconotus and Platyspathius, though it is not as long as in Antespathius.

The genus Leptorhaconotus was previously placed within the monotypic tribe Leptorhaconotini (van Achterberg 1984; Belokobylskij 1992). Recent molecular phylogenetic studies (Zaldívar-Riverón et al. 2008; Jasso-Martínez et al. 2019) consistently showed that it actually belongs to the Rhaconotini. However, Leptorhaconotus species are characterized by various exclusive morphological features (see remarks in the Leptorhaconotus redescription), which led us to maintain for them the suprageneric name Leptorhaconotina. 
A morphological feature that occurs in species of several rhaconotine genera (Arhaconotus, Bathycentor, Euryphrymnus, Ipodoryctes, Mimipodoryctes, Neorhaconotus, Platyspathius, Rhaconotinus, Rhaconotus and Troporhaconotus) is the presence of distinctly delineated basal (anterior) and/or apical (posterior) areas on the second metasomal tergite (Belokobylskij 2001). Such basal area usually is short and predominantly isolated from other tergite surface only different types of sculpture (Ipodoryctes and Mimipodoryctes), but sometimes it is wide and delineated by a deep and curved transverse groove (Arhaconotus, Bathycentor and Euryphrymnus) (Belokobylskij 2000, 2001). An apical lenticular or (rarely) subparallel area on the second tergite is also present in species of five genera (Arhaconotus, Neorhaconotus and numerous species of Rhaconotinus, Platyspathius and Troporhaconotus), being delineated anteriorly by a transverse, curved and usually crenulate groove, and posteriorly by a second suture. Interestingly, the latter anterior groove on the apical area, though usually shallow and wide, is also present in some species of Rhaconotus (including the genotype Rh. aciculatus Ruthe). Narrow apical areas on the second tergite often delineated anteriorly by different types of sculpture (usually striate before smooth area) are present in various species of Ipodoryctes and Mimipodoryctes (Belokobylskij 2001; Belokobylskij \& Chen 2004).

Members of the Rhaconotini are also characterised by some variable but important features in fore wing venation. In previous classifications, the main diagnostic character for this group was the interstitial position of parallel vein (CU1a) in the fore wing (Belokobylskij, 1992; van Achterberg \& Shaw, 2010). However, this feature is already not present in species of Bathycentor, Ipodoryctes, Mimipodoryctes, Rhacontsira and some species of Rhaconotinus, where the parallel vein (CU1a) arises before the middle or sometimes behind middle of distal vein (3-CU1) of brachial (first subdiscal) cell. Moreover, in species of the monotypic Grangerdoryctes and Pseudorhaconotus there is a loss of the second radiomedial vein $(\mathrm{r}-\mathrm{m})$, which is always present in the remaining members of the Rhaconotini.

This new generic classification and species composition update for the Rhaconotini will serve as a basis to improve the generic classification of other tribes within the Doryctinae.

\section{Acknowledgements}

We are very thankful to the former and current curators of the Natural History Museums in Berlin, Budapest, Canberra, Edinburgh, Helsinki, London, Madrid, Munich, Müncheberg, Ottawa, Paris, Stockholm and Washington for their valuable help and support during our visits to these museums and for their loan of specimens, including type material. We also thank to Olivia Evangelista (ANIC, Canberra) for sending digital images of the holotype of Neorhaconotus rieki. The authors are also grateful to Dr Yves Braet (Gembloux, Belgium) and an unknown reviewer for their very useful comments on the first version of the manuscript. This work was in part funded by grants given by of the Russian Foundation for Basic Research (project No. 19-04-00027) and the Russian State Research Project No. AAAA-A19-119020690101-6 to SAB, and by a grant given by Universidad Nacional Autónoma de México, Dirección General de Asuntos del Personal Académico (UNAM-DGAPA) (Convocatoria 2019, proyecto PAPIIT No 201119) to AZR.

\section{References}

Belokobylskij S.A. 1988a. Two new species of Rhaconotus Ruthe (Hymenoptera, Braconidae) from southeast Asia. Proceedings of the Zoological Institute of the USSR Academy of Sciences 178: 98-103. [In Russian.]

Belokobylskij S.A. 1988b. Three new braconid species of the subfamily Doryctinae (Hymenoptera, Braconidae) from Primorskiy Territory. Zoologicheskiy Zhurnal 67 (1): 625-630. [In Russian.] 
BELOKOBYLSKIJ S.A. \& ZALDÍVAR-RIVERÓN A., Reclassification of the Rhaconotini

Belokobylskij S.A. 1990a. Review of braconid wasps of the genus Rhaconotus Ruthe (Hymenoptera, Braconidae) of the Palaearctic Region. Entomologicheskoe Obozrenie 69 (1): 144-163. [In Russian.]

Belokobylskij S.A. 1990b. Tribe Rhaconotini (Hymenoptera, Braconidae, Doryctinae): a new genus from Vietnam. Proceedings of the Zoological Institute of the USSR Academy of Sciences 209: 141-144. [In Russian.]

Belokobylskij S.A. 1992. On the classification and phylogeny of the braconid wasps subfamilies Doryctinae and Exothecinae (Hymenoptera, Braconidae) Part 1. On the classification. Entomologicheskoe Obozrenie 71 (4): 900-927. [In Russian. English translation: Entomological Review 72 (6): 109-137.]

Belokobylskij S.A. 1994a. Addition to revision of the genus Rhaconotus Ruthe (Hymenoptera, Braconidae, Doryctinae) of the Palaearctic Region. Entomologicheskoe Obozrenie 73 (2): 340-351. [In Russian.]

Belokobylskij S.A. 1994b. A new Oriental species of the genus Ipodoryctes Granger (Hymenoptera, Braconidae, Doryctinae). Zoosystematica Rossica 3 (1): 129-140.

Belokobylskij S.A. 1995. Two new genera and two new subgenera of the braconid wasps from Old Word (Hymenoptera: Braconidae: Exothecinae, Doryctinae). Zoologische Mededlungen 69 (3): 37-52.

Belokobylskij S.A. 1996a. A contribution to the knowledge of the Doryctinae of Taiwan (Hymenoptera: Braconidae). Zoosystematica Rossica 5 (1): 153-191.

Belokobylskij S.A. 1996b. Parasitism on the beetles (Coleoptera) as important stage in the evolution of braconide wasps (Hymenoptera, Braconidae). Part II. Entomologicheskoe Obozrenie 75 (4): 790-814. [In Russian.]

Belokobylskij S.A. 1998a. Three new genera of the Braconidae (Hymenoptera) from East Asia. Far Eastern Entomologist 54: 1-14.

Belokobylskij S.A. 1998b. Fam. Braconidae. Subfam. Doryctinae. In: Lehr P.A. (ed.). Key to Insects of the Russian Far East. Neuropteroidea, Mecoptera, Hymenoptera 4 (3): 50-109. Dal'nauka, Vladivostok. [In Russian.]

Belokobylskij S.A. 2000. Two new Oriental-Australian genera of Doryctinae (Hymenoptera: Braconidae) with immovably fused first three metasomal tergites. Russian Entomological Journal 9 (4): 345-351.

Belokobylskij S.A. 2001. New species of the genera Rhaconotus Ruthe, Ipodoryctes Granger and Arhaconotus Blkb. from the Oriental Region (Hymenoptera: Braconidae, Doryctinae). Zoosystematica Rossica 10 (1): 101-162.

Belokobylskij S.A. (2004) 2005. Two new taxa of subtribe Rhaconotina (Hymenoptera: Braconidae, Doryctinae, Doryctini) from Africa, with a key to subtribe genera. Annales de la Société entomologique de France (N.S.) 40 (2): 205-210. https://doi.org/10.1080/00379271.2004.10697418

Belokobylskij S.A. 2018. Review of the braconid wasp genus Bathycentor Saussure, 1892 (Hymenoptera, Braconidae: Doryctinae) with description of a new species from Central Africa. Entomologicheskoe Obozrenie 97 (3): 502-515. [In Russian. English translation: Entomological Review 98 (6): 765-775. https://doi.org/10.1134/S0013873818060143]

Belokobylskij S.A. 2019. Some taxonomical corrections and new faunistic records of the species from the family Braconidae (Hymenoptera) in the fauna of Russia. Proceedings of the Russian Entomological Society 90: 33-53. https://doi.org/10.47640/1605-7678_2019_90_33

Belokobylskij S.A. \& Austin A.D. 2013. New species of flightless doryctine parasitic wasps (Hymenoptera: Braconidae: Doryctinae) from Australia and New Zealand. Australian Journal of Entomology 52 (4): 338-355. https://doi.org/10.1111/aen.12042 
Belokobylskij S.A. \& Chen X.-X. 2004. The species of the genus Rhaconotus Ruthe, 1854 (Hymenoptera: Braconidae: Doryctinae) from China with a key to species. Annales Zoologici 54 (2): 319-359.

Belokobylskij S.A. \& Ku D.-S. 2001. A new species of the genus Platyspathius Viereck (Hymenoptera, Braconidae, Doryctinae) from Korea. Insecta Koreana 18 (1): 37-40.

Belokobylskij S.A. \& Maeto K. 2006. Review of ten genera of the subfamily Doryctinae (Hymenoptera: Braconidae) new for Japan. Annales Zoologici 56 (4): 587-664.

Belokobylskij S.A. \& Maeto K. 2009. Doryctinae (Hymenoptera, Braconidae) of Japan. Fauna mundi. Vol. 1. Warshawska Drukarnia Naukowa, Warszawa.

Belokobylskij S.A. \& Tobias V.I. 1986. Subfam. Doryctinae. In: Medvedev G.S. (ed.) Opredelitel' nasekomykh evropeyskoy chasti SSSR. Pereponchatokrylye [Keys to the insects of the European part of USSR. Hymenoptera] 3 (4): 21-72. Nauka, Leningrad. [In Russian.]

Belokobylskij S.A. \& Villemant C. 2015. Platyspathius picardi sp. nov., a new European species of the genus Platyspathius Viereck, 1911 (Hymenoptera: Braconidae: Doryctinae). Zoosystematica Rossica 24 (1): 113-120. https://doi.org/10.31610/zsr/2015.24.1.113

Belokobylskij S.A., Iqbal M. \& Austin A.D. 2004. Systematics, distribution and diversity of the Australasian doryctine wasps (Hymenoptera, Braconidae, Doryctinae). Records of the South Australian Museum Monograph Series 8: 1-150.

Belokobylskij S.A., Samaca-Saez. E. \& Zaldívar-Riverón A. 2015. Mexiare gen. nov., a new Doryctinae genus (Hymenoptera: Braconidae) from Mexico with fused first and second metasomal tergites. Zootaxa 3914 (2): 122-130. https://doi.org//10.11646/zootaxa.3914.2.2

Belokobylskij S.A., Kotenko A.G. \& Samartsev K.G. 2019. Family Braconidae. In: Belokobylskij S.A., Samartsev K.G. \& Il'inskaya A.S. (eds) Annotated catalogue of the Hymenoptera of Russia. Volume II. Apocrita: Parasitica. Proceedings of the Zoological Institute of the Russian Academy of Sciences. 323 Supplement 8: 200-329. https://doi.org/10.31610/trudyzin/2019.supl.8.5

Cameron P. 1909. On some new Bornean species of Braconidae. Societas Entomologica 24: 114.

Cameron P. 1910. On some Asiatic species of the subfamilies Spathiinae, Doryctinae, Rhogadinae, Cardiochilinae and Macrocentrinae in the Royal Berlin Zoological Museum. Wiener Entomologische Zeitschrift 29: 93-100. https://doi.org/10.5962/bhl.part.23337

Chen J. \& Shi Q. 2004. Systematic Study on Doryctinae of China (Hymenoptera: Braconidae). Fujian Sciences and Technology Publishing House, Fuzhou.

Docavo Alberti I. 1960. Especies nuevas de Braconinae de España (Hym. Braconidae). Eos 36: 25-39.

Enderlein G. 1912. Zur Kenntnis der Spathiinen und einiger verwandter Gruppen. Archiv für Naturgeschichte 78 (A): 1-37.

Fahringer J. 1928. Opuscula braconologica. Band II. Aethiopische Region. Lieferung 1-3. Opuscula braconologica: $1-224$.

Foerster A. (1862) 1863. Synopsis der Familien und Gattungen der Braconiden. Verhandlungen des Naturhistorischen Vereins der Preussischen Rheinlande und Westfalens 19: 225-288.

Giraud J. 1869. Observations hyménoptèrologiques. Annales de la Société entomologique de France 4 (9): 469-488.

Granger C. 1949. Braconides de Madagascar. Mémoires de l'Institut scientifique de Madagascar. (A), 2: $1-428$. 
Hedqvist K.J. 1965. Braconidae from the Cape Verde Islands. Commentationes Biologicae (Helsinki) 28: $1-28$.

Jasso-Martínez J.M., Belokobylskij S.A. \& Zaldívar-Riverón A. 2019. Molecular phylogenetics and evolution of generic diagnostic morphological features in the doryctine wasp tribe Rhaconotini (Hymenoptera: Braconidae). Zoologischer Anzeiger 279: 164-171.

https://doi.org/10.1016/j.jcz.2019.02.002

Long K.D. \& Belokobylskij S.A. 2011. Vietnamese species of the genus Spathius Nees (Hymenoptera: Braconidae: Doryctinae) with reduced first radiomedial vein of the forewing. American Museum Novitates 3721: 1-26. https://doi.org/10.1206/3721.2

Marsh P.M. 1965. The Nearctic Doryctinae I. A review of the subfamily with a taxonomic revision of the tribe Hecabolini (Hymenoptera: Braconidae). Annals of the Entomological Society of America 58: 668-699. https://doi.org/10.1093/aesa/58.5.668

Marsh P.M. 1976. The Nearctic Doryctinae, X. The genus Rhaconotus Ruthe (Hymenoptera: Braconidae). Proceedings of the Entomological Society of Washington 78 (4): 389-403.

Marsh P.M. 2002. The Doryctinae of Costa Rica (excluding the genus Heterospilus). Memoirs of the American Entomological Institute 70: 1-319.

Nixon G.E.J. 1941. The Indian and African species of Rhaconotus Ruthe (Hym., Braconidae). Annals and Magazine of Natural History 42 (7): 473-503. https://doi.org/10.1080/03745481.1941.9723712

Nixon G.E.J. 1943. A revision of the Spathiinae of the Old World (Hymenoptera, Braconidae). Transactions of the Royal Entomological Society of London 93: 173-495. https://doi.org/10.1111/j.1365-2311.1943.tb00434.x

Qanh N.T. \& Long K.D.2019. New species of the Rhaconotus jacobsoni group (Hymenoptera, Braconidae, Doryctinae) from Vietnam. ZooKeys 853: 37-55. https://doi.org/10.3897/zookeys.853.33938

Quicke D.L.J. 1996. First record of Leptorhaconotus Granger (Hymenoptera: Braconidae) from South Africa, with the description of a remarkable new species and a discussion of the subfamilian placement of the genus. African Entomology 4 (2): 111-116.

Quicke D.L.J. \& van Achterberg C. 1990. Phylogeny of the subfamilies of the family Braconidae (Hymenoptera: Ichneumonidae). Zoologische Verhandelingen 258: 1-95.

Saussure H. de. (1890-1891) 1892. Hymenopteres. In: Grandidier A. (ed.) Histoire physique, naturelle et politique de Madagascar. 20 (1): Hyménoptères: 3-21. Imprimérie nationale, Paris. https://doi.org/10.5962/bhl.title.1599

Ruthe, 1854. Beiträge zur Kenntnis der Braconiden. Stettiner Entomologische Zeitung 15: 343-355.

Shenefelt R.D. \& Marsh P.M. 1976. Braconidae 9. Doryctinae. In: Ferriere Ch. \& van der Vecht J. (eds) Hymenopterorum Catalogus (nova editio) 13: 1263-1424.

Szépligeti G. 1908. E. Jacobons'sche Hymenopteren aus Semarang (Java). Evaniden, Braconiden und Ichneumoniden. Notes from the Leyden Museum 29: 209-260.

Szépligeti G. 1914. Afrikanische Braconiden des Königl. Zoologischen Museums in Berlin. Mitteilungen aus dem Zoologischen Museum in Berlin 7: 153-230.

Tang P., He J.-H. \& Chen X.-X. 2010. The genus Arhaconotus Belokobylskij (Hymenoptera, Braconidae, Doryctinae) from China, with description of a new species. ZooKeys 61: 63-68.

https://doi.org/10.3897/zookeys.61.488 
Tang P., Zhu L.-L., He J.-H. \& Chen X.-X. 2011. The genus Ipodoryctes Granger, 1949 (Hymenoptera: Braconidae, Doryctinae) from China. Zootaxa 2784: 1-19. https://doi.org/10.11646/zootaxa.2784.1.1

Tobias V. I. 1964. New species and genus of braconids (Hymenoptera, Braconidae) from Kazakhstan. Proceedings of the Zoological Institute 34: 177-234.

van Achterberg C. 1984. Essay on the phylogeny of the Braconidae (Hymenoptera: Ichneumonoidea). Entomologisk Tidskrift 105: 41-58.

van Achterberg C. 1993. Illustrated key to the subfamilies of the Braconidae (Hymenoptera: Ichneumonoidea). Zoologische Verhandelingen 283: 1-189.

van Achterberg C. 2003. The West Palaearctic species of the genera Gildoria Hedqvist and Platyspathius Viereck, with keys to the species (Hymenoptera: Braconidae: Doryctinae). Zoologische Mededelingen 77 (15): 267-290.

van Achterberg C. \& Shaw M.R. 2010. Pseudorhaconotus enervatus, a new genus and species from Spain (Hymenoptera: Braconidae: Doryctinae). Zoologische Mededelingen 84 (1-9): 1-5.

Viereck H.L. 1911. Descriptions of six new genera and thirty-one new species of Ichneumon flies. Proceedings of the United States National Museum 40 (1812): 173-196. https://doi.org/10.5479/si.00963801.1812.173

Viereck H.L. 1914. Type species of the genera of ichneumon flies. United States National Museum Bulletin 83: 1-186. https://doi.org/10.5479/si.03629236.83.1

Yu D.S.K., van Achterberg C. \& Horstmann K. 2016. Taxapad 2016, Ichneumonoidea 2015. Database on flash-drive. Nepean, Ontario.

Zaldivar-Riverón A., Belokobylskij S.A., León-Regagnon V., Martinez J.J., Briceno R. \& Quicke D.L.J. 2007. A single origin of gall association in a group of parasitic wasps with disparate morphologies. Molecular Phylogenetics and Evolution 44: 981-999. https://doi.org/10.1016/j.ympev.2007.05.016

Zaldívar-Riverón A., Belokobylskij S.A., León-Regagnon V., Briceño R. \& Quicke D.L.J. 2008. Molecular phylogeny and historical biogeography of the cosmopolitan parasitic wasp subfamily Doryctinae (Hymenoptera: Braconidae). Invertebrate Systematics 22: 345-363.

https://doi.org/10.1071/IS07028

Manuscript received: 15 July 2020

Manuscript accepted: 9 November 2020

Published on: 30 March 2021

Topic editor: Nesrine Akkari

Section editor: Gavin Broad

Desk editor: Jeroen Venderickx

Printed versions of all papers are also deposited in the libraries of the institutes that are members of the EJT consortium: Muséum national d'histoire naturelle, Paris, France; Meise Botanic Garden, Belgium; Royal Museum for Central Africa, Tervuren, Belgium; Royal Belgian Institute of Natural Sciences, Brussels, Belgium; Natural History Museum of Denmark, Copenhagen, Denmark; Naturalis Biodiversity Center, Leiden, the Netherlands; Museo Nacional de Ciencias Naturales-CSIC, Madrid, Spain; Real Jardín Botánico de Madrid CSIC, Spain; Zoological Research Museum Alexander Koenig, Bonn, Germany; National Museum, Prague, Czech Republic. 